Supporting Information

\title{
C-H Functionalization of Benzothiazoles via Thiazol-2-yl-phosphonium Intermediates
}

You Zi, Fritz Schömberg, Konrad Wagner and Ivan Vilotijevic*

Institute of Organic Chemistry and Macromolecular Chemistry, Friedrich Schiller University Jena, Humboldtstr. 10, 07743 Jena, Germany

*E-mail: ivan.vilotijevic@uni-jena.de 


\section{Contents}

Conditions optimization




\section{General remarks}

All the chemicals that are not mentioned in the subsequent parts were purchased from Merck, Alfa Aesar, Acros Organics, Fluorochem or TCl and used without further purification. The solvents if needed were dried according to standard conditions. For column chromatography flash column or CombiFlach (Teledyne Isco) device was used and $\operatorname{TLC}\left(\mathrm{SiO}_{2}, 60 \mathrm{M}\right.$, pore size $\left.0.04-0.063 \mathrm{~mm}\right)$, products of MacheryNagel were used. The TLC-glass-plates DURASIL consisted of a $0.25 \mathrm{~mm}$ layer of silica 60 with Fluorescence indicator UV254. TLCs were checked under UV-light (254 nm or $365 \mathrm{~nm}$ ) and stained with an aq. $\mathrm{KMnO}_{4}$-solution, PMA-stain, DNP or PAA. All ${ }^{1} \mathrm{H},{ }^{13} \mathrm{C},{ }^{19} \mathrm{~F}$ and ${ }^{31} \mathrm{P}$ NMR spectra were measured with a BRUKER $250\left({ }^{13} \mathrm{C}\right)$, BRUKER Fourier $300\left({ }^{1} \mathrm{H},{ }^{13} \mathrm{C}\right)$ or a BRUKER Avance 400 spectrometer $\left({ }^{1} \mathrm{H},{ }^{13} \mathrm{C},{ }^{19} \mathrm{~F}\right.$ and

$\left.{ }^{31} \mathrm{P}\right)$. The chemical shift of each signal was registered in ppm. For ${ }^{1} \mathrm{H}$ and ${ }^{13} \mathrm{C}$ measurements, the chemical shift refers to TMS, showing a signal at $0 \mathrm{ppm}$. As an internal standard, the remaining protons or respectively the carbons of the corresponding deuterated solvent were used $\left(\mathrm{CDCl}_{3}, 7.26 \mathrm{ppm}\left({ }^{1} \mathrm{H}-\mathrm{NMR}\right)\right.$, $\left.77.16 \mathrm{ppm}\left({ }^{13} \mathrm{C}-\mathrm{NMR}\right)\right)$. The chemical shift of the fluorine NMR was determined indirectly. For carbon spectra, a broadband decoupling was performed. High-resolution mass spectra (HRMS) were measured with El or ESI ionisation by the MS plattform. A chromatographic purification was performed before each measurement. The Thermo Q-Exactive plus device for ESI-mass spectra was coupled to a binary UHPLC system. For El-measurement, a GC-system was coupled to the Thermo Q-Exactive GC device. All the IRs were measured using the Shimadzu IR-Affinity-1 (FTIR) device. 


\section{Conditions optimization}

\begin{tabular}{|c|c|c|c|c|c|}
\hline Entry & 2a (equiv.) & Base (equiv.) & Solvent & Temp. $\left({ }^{\circ} \mathrm{C}\right)$ & Yield $^{[\mathrm{a}]}$ \\
\hline 1 & 1.0 & $\mathrm{NaH}(1.0)$ & THF & $0{ }^{\circ} \mathrm{C}-\mathrm{rt}$ & 55 \\
\hline 2 & 1.0 & n-BuLi (1.0) & THF & $-78^{\circ} \mathrm{C}-\mathrm{rt}$ & 44 \\
\hline 3 & 1.0 & LDA (1.0) & THF & $-78^{\circ} \mathrm{C}-\mathrm{rt}$ & 29 \\
\hline 4 & 1.0 & NaHMDS (1.0) & THF & $-78^{\circ} \mathrm{C}-\mathrm{rt}$ & 68 \\
\hline 5 & 1.0 & $i-\operatorname{PrMgBr}(1.0)$ & THF & $-78^{\circ} \mathrm{C}-\mathrm{rt}$ & Trace \\
\hline 6 & 1.0 & $t$-BuOK (1.0) & THF & $-78^{\circ} \mathrm{C}-\mathrm{rt}$ & Trace \\
\hline 7 & 1.0 & $\mathrm{Et}_{3} \mathrm{~N}(1.0)$ & THF & $\mathrm{rt}$ & Trace \\
\hline 8 & 1.0 & $\mathrm{DBU}(1.0)$ & THF & $\mathrm{rt}$ & 34 \\
\hline 9 & 1.0 & DMAP (1.0) & THF & $\mathrm{rt}$ & Trace \\
\hline 10 & 1.0 & DABCO (1.0) & THF & $\mathrm{rt}$ & Trace \\
\hline 11 & 1.0 & $\mathrm{NaH}(1.0)$ & $\mathrm{Et}_{2} \mathrm{O}$ & $0^{\circ} \mathrm{C}-\mathrm{rt}$ & 55 \\
\hline 12 & 1.0 & $\mathrm{NaH}(1.0)$ & Dioxane & $0^{\circ} \mathrm{C}-\mathrm{rt}$ & 29 \\
\hline 13 & 1.0 & $\mathrm{NaH}(1.0)$ & n-Hexane & $0^{\circ} \mathrm{C}-\mathrm{rt}$ & 16 \\
\hline 14 & 1.0 & $\mathrm{NaH}(1.0)$ & Toluene & $0{ }^{\circ} \mathrm{C}-\mathrm{rt}$ & 56 \\
\hline 15 & 1.0 & $\mathrm{NaH}(1.0)$ & DCE & $0{ }^{\circ} \mathrm{C}-\mathrm{rt}$ & Trace \\
\hline 16 & 1.0 & $\mathrm{NaH}(1.0)$ & MTBE & $0^{\circ} \mathrm{C}-\mathrm{rt}$ & 11 \\
\hline 17 & 1.0 & $\mathrm{NaH}(1.0)$ & Chlorobenzene & $0{ }^{\circ} \mathrm{C}-\mathrm{rt}$ & 37 \\
\hline 18 & 1.0 & $\mathrm{NaH}(1.0)$ & $\mathrm{PhCF}_{3}$ & $0{ }^{\circ} \mathrm{C}-\mathrm{rt}$ & 40 \\
\hline 19 & 1.25 & $\mathrm{NaH}(1.25)$ & THF & $0{ }^{\circ} \mathrm{C}-\mathrm{rt}$ & 55 \\
\hline 20 & 1.5 & $\mathrm{NaH}(1.5)$ & THF & $0{ }^{\circ} \mathrm{C}-\mathrm{rt}$ & $80^{[b][c]}$ \\
\hline 21 & 2 & $\mathrm{NaH}(2.0)$ & THF & $0{ }^{\circ} \mathrm{C}-\mathrm{rt}$ & 75 \\
\hline
\end{tabular}

[a] Yields based on NMR with a certain internal standard; [b] Isolated yield; [c] 82\% on $1 \mathrm{mmol}$ scale. 


\section{Synthesis of Phosphonium salts}

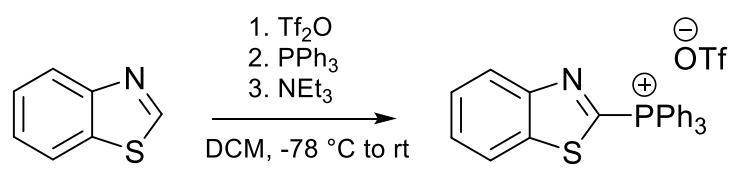

An oven dried round bottom flask equipped with a stirring bar was charged with the heterocycle (1.0 eq) and placed under a nitrogen atmosphere. Appropriate amount of DCM was added to prepare $0.1 \mathrm{M}$ solution of the starting substituted thiazole and the reaction vessel was cooled to $-78{ }^{\circ} \mathrm{C} . \mathrm{Tf}_{2} \mathrm{O}(1.0 \mathrm{eq})$ was added dropwise over 5 minutes. The reaction was stirred for 30 minutes followed by the addition of $\mathrm{PPh}_{3}$ (1.1 eq) in one portion. The reaction was subjected to three rapid cycles of vacuum/nitrogen backfill and was stirred for another 30 minutes at $-78^{\circ} \mathrm{C}$. NEt $\mathrm{N}_{3}(1.0 \mathrm{eq})$ was added dropwise via syringe and subsequently the reaction was warmed up to room temperature while stirring (approximately 15-30 minutes). The reaction was quenched by saturated $\mathrm{NH}_{4} \mathrm{Cl}$ solution (approximately the same volume as DCM) and the mixture was transferred to a separatory funnel. The mixture was diluted with DCM and the resulting organic layer was washed three times with $\mathrm{H}_{2} \mathrm{O}$. The organic layer was dried $\left(\mathrm{Na}_{2} \mathrm{SO}_{4}\right)$, filtered and concentrated under vacuo. An excess of chilled $\mathrm{Et}_{2} \mathrm{O}\left(0^{\circ} \mathrm{C}\right)$ was added to the concentrated solution which was first placed in an ultrasonic bath (approximately 15-30 minutes) then placed in a -20 ${ }^{\circ} \mathrm{C}$ refrigerator overnight. The resulting suspension was filtered and the solid was washed with chilled $\mathrm{Et}_{2} \mathrm{O}\left(0^{\circ} \mathrm{C}\right)$ and dried in vacuo to provide the pure phosphonium salt or purified by automated column chromatography using silica gel and a gradient of $0 \%$ to $5 \%$ of methanol in DCM.

\section{benzo[d]thiazol-2-yltriphenylphosphonium trifluoromethanesulfonate $(2 \mathrm{a})^{[1]}$}

An oven dried round bottom flask equipped with a stirring bar was charged with the benzothiazole (1.35 g, $10 \mathrm{mmol}$ eq.) and dissolved in $\mathrm{DCM}(100 \mathrm{~mL})$. The reaction mixture was cooled to $-78{ }^{\circ} \mathrm{C}$ and $\mathrm{Tf}_{2} \mathrm{O}$ $(2.82 \mathrm{~g}, 1.68 \mathrm{~mL}, 10 \mathrm{mmol})$ was added dropwise over 5 minutes. The reaction mixture was stirred for 30 minutes followed by the addition of $\mathrm{PPh}_{3}(2,89 \mathrm{~g}, 11 \mathrm{mmol})$ in one portion. The mixture was stirred for another 30 minutes at $-78{ }^{\circ} \mathrm{C}$. $\mathrm{NEt}_{3}(1.01 \mathrm{~g}, 1.39 \mathrm{~mL}, 10 \mathrm{mmol})$ was added dropwise via syringe and the reaction mixture was warmed up to room temperature over 15 minutes. The reaction was quenched by addition of saturated $\mathrm{NH}_{4} \mathrm{Cl}$ solution and the mixture was transferred to a separatory funnel. Additional portion of DCM was added, and the organic was separated from the aqueous phase. Organic phase was washed three times with $\mathrm{H}_{2} \mathrm{O}$, dried over anhydrous $\mathrm{Na}_{2} \mathrm{SO}_{4}$, filtered and concentrated under reduced pressure. An excess of chilled $\mathrm{Et}_{2} \mathrm{O}\left(0^{\circ} \mathrm{C}\right)$ was added to the concentrated solution which was first placed in an ultrasonic bath (approximately $15-30$ minutes) then kept at $-20{ }^{\circ} \mathrm{C}$ overnight. The resulting suspension was filtered and the solid was washed with chilled $\mathrm{Et}_{2} \mathrm{O}\left(0^{\circ} \mathrm{C}\right)$ and dried in vacuo to provide the pure phosphonium salt $(4.74 \mathrm{~g}, 87 \%)$.

Yield: $87 \%$ (4.74 g, white solid, precipitated in diethyl ether). ${ }^{1} \mathbf{H}-\mathbf{N M R}(400 \mathrm{MHz}$, $\left.\mathrm{CDCl}_{3}\right) \delta 8.36(\mathrm{dd}, \mathrm{J}=7.4,1.3 \mathrm{~Hz}, 1 \mathrm{H}), 8.16(\mathrm{dd}, \mathrm{J}=7.3,1.3 \mathrm{~Hz}, 1 \mathrm{H}), 8.00-7.92$ (m, 3H), $7.87-7.76(\mathrm{~m}, 12 \mathrm{H}), 7.76-7.67(\mathrm{~m}, 2 \mathrm{H}) .{ }^{13} \mathrm{C}-\mathrm{NMR}\left(101 \mathrm{MHz}, \mathrm{CDCl}_{3}\right) \delta$ $155.9,155.7,152.3,151.1,137.5,136.6,136.6,134.6,134.5,131.0,130.9$,

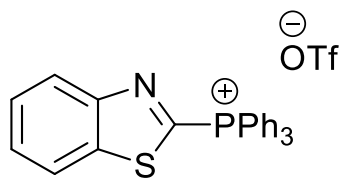
$129.2,128.6,125.6,122.6,116.8,115.9$. 
(6-methylbenzo[d]thiazol-2-yl)triphenylphosphonium trifluoromethanesulfonate (2b)

Yield: $68 \%$ (2.12 g, pale white solid, precipitated in diethyl ether). ${ }^{1} \mathbf{H}$ NMR $\left(400 \mathrm{MHz}, \mathrm{CDCl}_{3}\right) \delta 8.23(\mathrm{~d}, J=8.5 \mathrm{~Hz}, 1 \mathrm{H}), 8.00-7.90(\mathrm{~m}, 4 \mathrm{H}), 7.86-7.77$ $(\mathrm{m}, 12 \mathrm{H}), 7.56(\mathrm{dd}, J=8.6,1.7 \mathrm{~Hz}, 1 \mathrm{H}), 2.58(\mathrm{~s}, 3 \mathrm{H}) .{ }^{13} \mathrm{C}$ NMR $(101 \mathrm{MHz}$, $\left.\mathrm{CDCl}_{3}\right) \delta 154.3,154.1,150.4,149.2,140.4,137.9,136.6,136.5,134.5,134.4$,

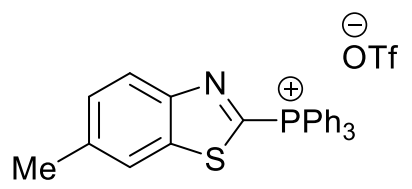
131.0, 130.9, 130.6, 125.1, 121.8, 117.0, 116.0, 21.9. ${ }^{31} \mathrm{P}$ NMR (162 MHz, $\left.\mathrm{CDCl}_{3}\right) \delta$ 17.94. ${ }^{19} \mathrm{~F}$ NMR (377 $\left.\mathrm{MHz}, \mathrm{CDCl}_{3}\right) \delta$-78.02. HRMS: [EI]: $\mathrm{m} / \mathrm{z}$ calculated for $\mathrm{C}_{26} \mathrm{H}_{21} \mathrm{NPS}^{+}[\mathrm{M}]^{+} 410.1127$, found 410.1124. IR (ATR): $v=3063,1589,1439,1589,1150,1111,1030,810 \mathrm{~cm}^{-1}$.

\section{(5-methoxybenzo[d]thiazol-2-yl)triphenylphosphonium trifluoromethanesulfonate (2c)}

Yield: $59 \%$ (1 $\mathrm{g}$, pale white solid, precipitated in diethyl ether). ${ }^{1} \mathbf{H}$ NMR $\left(400 \mathrm{MHz} \mathrm{CDCl}_{3}\right) \delta 8.02-7.92(\mathrm{~m}, 4 \mathrm{H}), 7.84-7.76(\mathrm{~m}, 13 \mathrm{H}), 7.34$ (dd, $J=$ 9.1, $2.5 \mathrm{~Hz}, 1 \mathrm{H}), 3.96(\mathrm{~s}, 3 \mathrm{H}) .{ }^{13} \mathrm{C}$ NMR $\left(101 \mathrm{MHz}, \mathrm{CDCl}_{3}\right) \delta 160.6,157.7$, $157.5,152.3,151.1,136.6,136.6,134.5,134.4,131.0,130.86,130.0$,<smiles>COc1ccc2sc([PbH])nc2c1</smiles>
122.6, 120.9, 116.9, 116.0, 106.1, 55.9. ${ }^{31} \mathrm{P}$ NMR $\left(162 \mathrm{MHz}, \mathrm{CDCl}_{3}\right) \delta 17.82 .{ }^{19} \mathrm{~F}$ NMR $\left(377 \mathrm{MHz}, \mathrm{CDCl}_{3}\right) \delta$ 78.03. HRMS: [EI]: $\mathrm{m} / \mathrm{z}$ calculated for $\mathrm{C}_{26} \mathrm{H}_{21}$ NOPS $^{+}[\mathrm{M}]^{+} 426.1076$, found 426.1065 . IR (ATR): $v=3055$, $1585,1439,1260,1146,1111,1026,729 \mathrm{~cm}^{-1}$.

\section{(6-fluorobenzo[d]thiazol-2-yl)triphenylphosphonium trifluoromethanesulfonate (2d)}

Yield: 54\% (1.7 g, pale white solid, precipitated in diethyl ether). ${ }^{1} \mathbf{H}$ NMR (400 $\left.\mathrm{MHz}, \mathrm{CDCl}_{3}\right) \delta 8.34(\mathrm{dd}, J=9.2,4.7 \mathrm{~Hz}, 1 \mathrm{H}), 8.00-7.91(\mathrm{~m}, 3 \mathrm{H}), 7.87-7.77(\mathrm{~m}$, $13 \mathrm{H}), 7.49-7.43(\mathrm{~m}, 1 \mathrm{H}) .{ }^{13} \mathrm{C}$ NMR $\left(101 \mathrm{MHz}, \mathrm{CDCl}_{3}\right) \delta 163.7,161.2,152.7$, $152.5,152.2,152.2,151.0,151.0,139.1,139.0,136.7,136.6,134.6,134.4$,<smiles>[18OH]Pc1nc2ccc(F)cc2s1</smiles>
131.1, 130.9, 127.2, 127.1, 118.3, 118.0, 116.6, 115.7, 108.9, 108.6. ${ }^{31} \mathrm{P}$ NMR (162 MHz, CDCl $\left.{ }_{3}\right) \delta 18.09$. ${ }^{19} \mathrm{~F}$ NMR (377 MHz, CDCl 3 ) $\delta$-78.04. HRMS: [El]: $\mathrm{m} / \mathrm{z}$ calculated for $\mathrm{C}_{25} \mathrm{H}_{18} \mathrm{FNPS}^{+}[\mathrm{M}]^{+}$414.0876, found 414.0871. IR (ATR): $v=3067,1601,1558,1466,1439,1261,1142,1107,1030,818 \mathrm{~cm}^{-1}$.

\section{(7-bromobenzo[d]thiazol-2-yl)triphenylphosphonium trifluoromethanesulfonate (2e)}

Yield: $42 \%$ (772 mg, pale white solid, precipitated in diethyl ether). ${ }^{1} \mathbf{H}$ NMR (400 $\left.\mathrm{MHz}, \mathrm{CDCl}_{3}\right) \delta 8.27(\mathrm{~d}, J=8.3 \mathrm{~Hz}, 1 \mathrm{H}), 7.94-7.87(\mathrm{~m}, 3 \mathrm{H}), 7.81-7.71(\mathrm{~m}, 13 \mathrm{H})$, $7.60(\mathrm{t}, J=8.1 \mathrm{~Hz}, 1 \mathrm{H}) .{ }^{13} \mathrm{C}$ NMR $\left(101 \mathrm{MHz}, \mathrm{CDCl}_{3}\right) \delta 155.3,155.1,153.3,152.1$, $140.5,136.9,136.8,134.6,134.5,131.8,131.2,131.1,130.0,129.0,128.2,124.8$, 116.3, 115.4, 114.1. ${ }^{31} \mathrm{P}$ NMR $\left(162 \mathrm{MHz}, \mathrm{CDCl}_{3}\right) \delta 14.60 .{ }^{19} \mathrm{~F} \mathrm{NMR}\left(377 \mathrm{MHz}, \mathrm{CDCl}_{3}\right)$

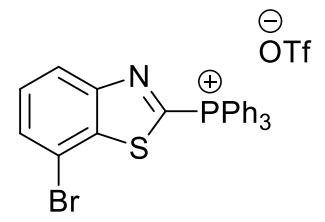
$\delta$-78.03. HRMS: [EI]: $\mathrm{m} / \mathrm{z}$ calculated for $\mathrm{C}_{25} \mathrm{H}_{18} \mathrm{BrNPS}^{+}[\mathrm{M}]^{+} 474.0075$, found 474.0071. IR (ATR): $v=$ $3063,1740,1439,1261,1146,1107,1030,914,795,729 \mathrm{~cm}^{-1}$. 
(5-bromobenzo[d]thiazol-2-yl)triphenylphosphonium trifluoromethanesulfonate (2f)

Yield: $63 \%$ (1.15 g, pale white solid, precipitated in diethyl ether). ${ }^{1} \mathbf{H}$ NMR $\left(400 \mathrm{MHz}, \mathrm{CDCl}_{3}\right) \delta 8.50(\mathrm{~d}, J=1.8 \mathrm{~Hz}, 1 \mathrm{H}), 8.12(\mathrm{~d}, J=8.7 \mathrm{~Hz}, 1 \mathrm{H}), 8.00-7.93$ $(\mathrm{m}, 3 \mathrm{H}), 7.84-7.76(\mathrm{~m}, 13 \mathrm{H}) .{ }^{13} \mathrm{C}$ NMR $\left(101 \mathrm{MHz}, \mathrm{CDCl}_{3}\right) \delta 156.7,156.5,154.7$, $153.5,136.7,136.7,136.5,134.6,134.5,132.3,131.1,130.9,128.0,124.1$,<smiles></smiles>
122.4, 122.4, 116.4, 115.5. ${ }^{31} \mathrm{P}$ NMR $\left(162 \mathrm{MHz}, \mathrm{CDCl}_{3}\right) \delta$ 18.42. ${ }^{19} \mathrm{~F}$ NMR $\left(377 \mathrm{MHz}, \mathrm{CDCl}_{3}\right) \delta-78.04$. HRMS: [EI]: $\mathrm{m} / \mathrm{z}$ calculated for $\mathrm{C}_{25} \mathrm{H}_{18} \mathrm{BrNPS}^{+}[\mathrm{M}]^{+}$474.0075, found 474.0073. IR (ATR): $V=3063,1585$, $1439,1261,1150,1107,1026,895,729 \mathrm{~cm}^{-1}$.

\section{(6-chlorobenzo[d]thiazol-2-yl)triphenylphosphonium trifluoromethanesulfonate $(2 \mathrm{~g})$}

Yield: $41 \%$ (117 mg, pale white solid, automated column chromatography with a gradient of $0 \%$ to $5 \%$ of methanol in DCM). ${ }^{1} \mathbf{H}$ NMR $\left(400 \mathrm{MHz}, \mathrm{CDCl}_{3}\right) \delta$ $8.59(\mathrm{~d}, J=2.1 \mathrm{~Hz}, 1 \mathrm{H}), 8.46(\mathrm{~d}, J=8.8 \mathrm{~Hz}, 1 \mathrm{H}), 8.05(\mathrm{td}, J=7.4,1.3 \mathrm{~Hz}, 3 \mathrm{H})$, $7.97-7.94(\mathrm{~m}, 6 \mathrm{H}), 7.90-7.85(\mathrm{~m}, 7 \mathrm{H}) .{ }^{13} \mathrm{C}$ NMR $\left(101 \mathrm{MHz}, \mathrm{CDCl}_{3}\right) \delta 155.6$, $154.5,154.4,154.3,139.3,136.8,136.8,135.3,135.2,133.9,131.2,131.1$,<smiles>Clc1ccc2nc([Pb])sc2c1</smiles>
129.5, 126.9, 123.3, 117.3, 116.4. ${ }^{31} \mathrm{P}$ NMR $\left(162 \mathrm{MHz}, \mathrm{CDCl}_{3}\right)$ ठ 18.17. ${ }^{19} \mathrm{~F}$ NMR $\left(377 \mathrm{MHz}, \mathrm{CDCl}_{3}\right) \delta-77.74$. HRMS: [EI]: $\mathrm{m} / \mathrm{z}$ calculated for $\mathrm{C}_{25} \mathrm{H}_{18} \mathrm{CINPS}^{+}[\mathrm{M}]^{+}$430.0581, found 430.0586. IR (ATR): $v=3063,1585$, $1439,1258,1150,1107,1030,822 \mathrm{~cm}^{-1}$.

(5-cyanobenzo[d]thiazol-2-yl)triphenylphosphonium trifluoromethanesulfonate (2h)

Yield: $63 \%$ (1.06 g, yellow solid, precipitated in diethyl ether). ${ }^{1} \mathbf{H}$ NMR (400 $\left.\mathrm{MHz}, \mathrm{CDCl}_{3}\right) \delta 8.63(\mathrm{~d}, J=1.5 \mathrm{~Hz}, 1 \mathrm{H}), 8.45(\mathrm{~d}, J=8.5 \mathrm{~Hz}, 1 \mathrm{H}), 8.01-7.94(\mathrm{~m}$, $3 \mathrm{H}), 7.85-7.79(\mathrm{~m}, 13 \mathrm{H}) .{ }^{13} \mathrm{C}$ NMR $\left(101 \mathrm{MHz}, \mathrm{CDCl}_{3}\right) \delta 157.0,155.8,154.9$, $154.7,141.8,136.9,136.8,134.7,134.6,131.2,131.0,130.4,129.8,128.6$,<smiles>N#Cc1ccc2sc([Pb])nc2c1</smiles>
$128.5,125.0,117.6,116.1,115.2,112.3 .{ }^{31} \mathrm{P}$ NMR $\left(162 \mathrm{MHz}, \mathrm{CDCl}_{3}\right) \delta 18.96 .{ }^{19} \mathrm{~F} \mathrm{NMR}\left(377 \mathrm{MHz}, \mathrm{CDCl}_{3}\right) \delta$ -78.10. HRMS: [EI]: $\mathrm{m} / \mathrm{z}$ calculated for $\mathrm{C}_{26} \mathrm{H}_{18} \mathrm{~N}_{2} \mathrm{PS}^{+}[\mathrm{M}]^{+} 421.0923$, found 421.0931. IR (ATR): $v=3063$, $1439,1258,1153,1107,1030,729 \mathrm{~cm}^{-1}$.

(6-cyanobenzo[d]thiazol-2-yl)triphenylphosphonium trifluoromethanesulfonate (2i)

Yield: 44\% (771 mg, yellow solid, precipitated in diethyl ether). ${ }^{1} \mathbf{H}$ NMR (400 $\left.\mathrm{MHz}, \mathrm{CDCl}_{3}\right) \delta 8.61(\mathrm{~d}, J=1.4 \mathrm{~Hz}, 1 \mathrm{H}), 8.46(\mathrm{~d}, J=8.6 \mathrm{~Hz}, 1 \mathrm{H}), 8.06-7.94(\mathrm{~m}$, $3 \mathrm{H}), 7.92(\mathrm{dd}, J=8.7,1.6 \mathrm{~Hz}, 1 \mathrm{H}), 7.88-7.75(\mathrm{~m}, 12 \mathrm{H}) .{ }^{13} \mathrm{C}$ NMR $(101 \mathrm{MHz}$, $\left.\mathrm{CDCl}_{3}\right) \delta 159.5,158.2,157.9,157.6,138.9,137.7,137.7,135.6,135.5,132.1$, $131.9,131.8,129.2,127.3,118.5,117.3,115.8,113.2 .{ }^{31} \mathrm{P}$ NMR (162 MHz,<smiles>N#Cc1ccc2nc([Pb](=O)[OH2+])sc2c1</smiles>
$\left.\mathrm{CDCl}_{3}\right) \delta$ 15.18. ${ }^{19} \mathrm{~F}$ NMR $\left(377 \mathrm{MHz}, \mathrm{CDCl}_{3}\right) \delta$-78.05. HRMS: [EI]: $\mathrm{m} / \mathrm{z}$ calculated for $\mathrm{C}_{26} \mathrm{H}_{18} \mathrm{~N}_{2} \mathrm{PS}^{+}[\mathrm{M}]^{+}$ 421.0923, found 421.0926. IR (ATR): $v=3067,1480,1261,1146,1107,1030,750 \mathrm{~cm}^{-1}$. 
(6-nitrobenzo[d]thiazol-2-yl)triphenylphosphonium trifluoromethanesulfonate (2j)

Yield: $24 \%$ (587 mg, yellow solid, precipitated in diethyl ether). ${ }^{1} \mathbf{H}$ NMR $\left(400 \mathrm{MHz}, \mathrm{CDCl}_{3}\right) \delta 9.47(\mathrm{~d}, J=2.3 \mathrm{~Hz}, 1 \mathrm{H}), 8.69-8.53(\mathrm{~m}, 2 \mathrm{H}), 8.06(\mathrm{td}, J=$ 7.4, $1.3 \mathrm{~Hz}, 3 \mathrm{H}), 8.02-7.96(\mathrm{~m}, 6 \mathrm{H}), 7.92-7.85(\mathrm{~m}, 6 \mathrm{H}) .{ }^{13} \mathrm{C}$ NMR $(101$ $\left.\mathrm{MHz}_{2} \mathrm{CDCl}_{3}\right) \delta 161.6,160.5,158.5,158.2,146.8,138.4,136.9,136.9,135.4$, $135.3,131.3,131.2,126.5,123.6,121.0,116.9,116.0 .{ }^{31} \mathrm{P}$ NMR $(162 \mathrm{MHz}$,<smiles>O=[N+]([O-])c1ccc2nc([Pb])sc2c1</smiles>
$\left.\mathrm{CDCl}_{3}\right) \delta$ 18.65. ${ }^{19} \mathrm{~F}$ NMR (377 MHz, $\left.\mathrm{CDCl}_{3}\right) \delta$-77.74. HRMS: [EI]: $\mathrm{m} / \mathrm{z}$ calculated for $\mathrm{C}_{25} \mathrm{H}_{18} \mathrm{~N}_{2} \mathrm{O}_{2} \mathrm{PS}^{+}[\mathrm{M}]^{+}$ 441.0821, found 441.0821. IR (ATR): $v=3075,1562,1516,1439,1342,1261,1146,1107,1030,895$, $841 \mathrm{~cm}^{-1}$.

\section{General procedure for the reaction of phosphonium salt with pronucleophiles}

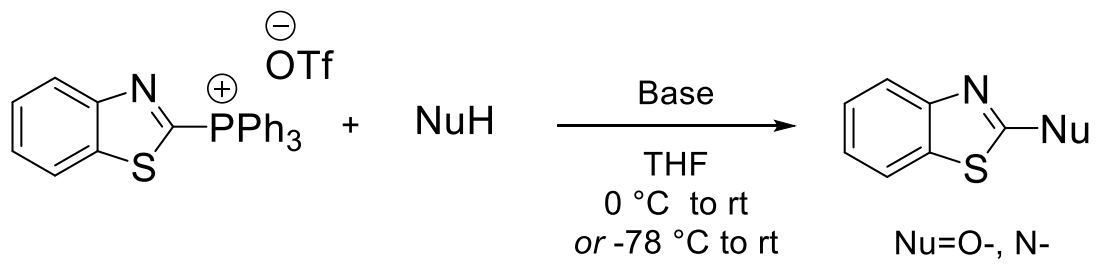

The base $(0.3 \mathrm{mmol})$ was added to the pronucleophile $(0.3 \mathrm{mmol})$ in dry THF $(0.1 \mathrm{M})$ under inert atmosphere at $0{ }^{\circ} \mathrm{C}(\mathrm{NaH})$ or $-78{ }^{\circ} \mathrm{C}(\mathrm{NaHMDS})$. After stirring the reaction for half an hour at room temperature $(\mathrm{NaH})$, the phosphonium salt $(0.2 \mathrm{mmol})$ was added subsequently at $0{ }^{\circ} \mathrm{C}(\mathrm{NaH})$ or $-78^{\circ} \mathrm{C}$ (NaHMDS) followed by three quick vacuum/nitrogen refills. The reaction mixture was warmed up to room temperature and quenched by addition of a small amount of water when it completed. The crude product was purified by column chromatography using silica gel with ethyl acetate in petroleum ether as the eluent or by an automated column chromatography with silica gel and a gradient of $0 \%$ to $20 \%$ ethyl acetate in petroleum ether.

\section{2-(benzyloxy)benzo[d]thiazole $(4 a)^{[2]}$}

$\mathrm{NaH}$ (37 mg, $1.5 \mathrm{mmol}$ ) was added to the benzylalcohol (162 mg, $155 \mu \mathrm{L}, 1.5 \mathrm{mmol}$ ) in dry THF (15 ml) at $0{ }^{\circ} \mathrm{C}$. After stirring the reaction for half an hour at room temperature, the phosphonium salt $2 \mathrm{a}(545 \mathrm{mg}$, $1.0 \mathrm{mmol}$ ) was added subsequently in one portion at $0{ }^{\circ} \mathrm{C}$ followed by three quick vacuum/nitrogen refills. The reaction mixture was warmed up to room temperature and quenched by addition of a small amount of water when it completed. The product $4 \mathrm{a}(198 \mathrm{mg}, 82 \%)$ was obtained via the purification by column chromatography with silica gel, $5 \%$ ethyl acetate in petroleum ether as the eluent.

Yield: $82 \%$ (198 mg, colourless oil, $5 \%$ ethyl acetate in petroleum ether). ${ }^{1} \mathbf{H}$ NMR $\left(300 \mathrm{MHz}, \mathrm{CDCl}_{3}\right) \delta 7.82-7.71(\mathrm{~m}, 1 \mathrm{H}), 7.67$ (ddd, $J=7.9,1.3,0.6 \mathrm{~Hz}$, $1 \mathrm{H}), 7.58-7.48(\mathrm{~m}, 2 \mathrm{H}), 7.47-7.36(\mathrm{~m}, 4 \mathrm{H}), 7.30-7.22(\mathrm{~m}, 1 \mathrm{H}), 5.63(\mathrm{~s}, 2 \mathrm{H})$. ${ }^{13} \mathrm{C}-\mathrm{NMR}\left(75 \mathrm{MHz}, \mathrm{CDCl}_{3}\right) \delta 172.7,149.3,135.2,132.0,128.7,128.7,128.5$,<smiles>c1ccc(COc2nc3ccccc3s2)cc1</smiles>
$126.0,123.6,121.3,120.9,73.4$. 


\section{2-((4-bromobenzyl)oxy)benzo[d]thiazole (4b)}

Yield: $99 \%$ (64 mg, colourless oil, 5\% ethyl acetate in petroleum ether). ${ }^{1} \mathrm{H}$ NMR (300 MHz, Chloroform-d) $\delta 7.73(\mathrm{dd}, J=8.2,1.3 \mathrm{~Hz}, 1 \mathrm{H}), 7.69-$ $7.61(\mathrm{~m}, 1 \mathrm{H}), 7.60-7.49(\mathrm{~m}, 2 \mathrm{H}), 7.45-7.16(\mathrm{~m}, 4 \mathrm{H}), 5.56(\mathrm{~s}, 2 \mathrm{H}) .{ }^{13} \mathrm{C}$

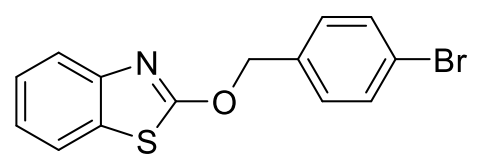
NMR $\left(75 \mathrm{MHz}, \mathrm{CDCl}_{3}\right) \delta 172.4,149.2,134.3,132.0,131.8,130.1,126.1$, 123.7, 122.7, 121.4, 120.9, 72.4. HRMS [EI]: $\mathrm{m} / \mathrm{z}$ calculated for $\mathrm{C}_{14} \mathrm{H}_{10} \mathrm{BrNOS}[\mathrm{M}]^{+} 318.9666$, found 318.9659. IR (ATR): $v=2924,1531,1439,1250,1215,1065,991,799 \mathrm{~cm}^{-1}$.

\section{2-([1,1'-biphenyl]-4-ylmethoxy)benzo[d]thiazole (4c)}

Yield: $73 \%$ (46 mg, colourless needles, 5\% ethyl acetate in petroleum ether). ${ }^{1} \mathrm{H}-\mathrm{NMR}\left(300 \mathrm{MHz}, \mathrm{CDCl}_{3}\right) \delta 7.78(\mathrm{dd}, J=8.0,1.1$ $\mathrm{Hz}, 1 \mathrm{H}), 7.72-7.56(\mathrm{~m}, 7 \mathrm{H}), 7.53-7.37(\mathrm{~m}, 4 \mathrm{H}), 7.28(\mathrm{td}, J=7.6$, $1.2 \mathrm{~Hz}, 1 \mathrm{H}), 5.68(\mathrm{~s}, 2 \mathrm{H}) .{ }^{13} \mathrm{C}-\mathrm{NMR}\left(75 \mathrm{MHz}, \mathrm{CDCl}_{3}\right) \delta 172.7,149.3$,

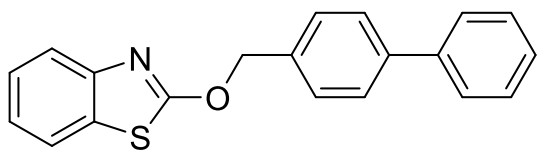
141.7, 140.6, 134.2, 132.1, 129.0, 128.9, 127.6, 127.4, 127.2, 126.1, 123.6, 121.6, 120.9, 73.1. HRMS [EI]: $\mathrm{m} / \mathrm{z}$ calculated for $\mathrm{C}_{20} \mathrm{H}_{15} \mathrm{NOS}[\mathrm{M}]^{+} 317.0874$, found 317.0869. IR (ATR): $v=3035,1739,1527,1365$, $1238,1211,918,825,725 \mathrm{~cm}^{-1}$.

\section{2-(3-phenylpropoxy)benzo[d]thiazole (4d)}

Yield: $63 \%$ (34 mg, colourless solid, 5\% ethyl acetate in petroleum ether). ${ }^{1} \mathrm{H}-\mathrm{NMR}\left(300 \mathrm{MHz}, \mathrm{CDCl}_{3}\right) \delta 7.69$ (ddd, $J=12.5,8.5,1.3 \mathrm{~Hz}, 2 \mathrm{H}$ ), $7.41-7.32(\mathrm{~m}, 2 \mathrm{H}), 7.29-7.15(\mathrm{~m}, 5 \mathrm{H}), 4.60(\mathrm{t}, J=6.4 \mathrm{~Hz}, 2 \mathrm{H}), 2.93-$ $2.77(\mathrm{~m}, 2 \mathrm{H}), 2.27-2.12(\mathrm{~m}, 2 \mathrm{H}) .{ }^{13} \mathrm{C}-\mathrm{NMR}\left(75 \mathrm{MHz}, \mathrm{CDCl}_{3}\right) \delta 172.9$,

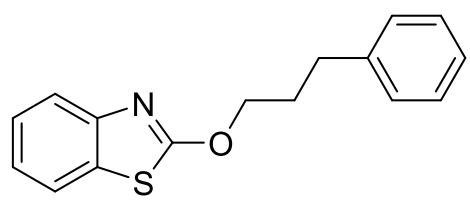
$149.4,141.0,131.9,128.5,128.5,126.1,126.0,123.5,121.3,120.8,71.2,32.0,30.5$. HRMS [EI]: $\mathrm{m} / \mathrm{z}$ calculated for $\mathrm{C}_{16} \mathrm{H}_{15} \mathrm{NOS}[\mathrm{M}]^{+} 269.0874$, found 269.0868. IR (ATR): $v=2924,1735,1527,1438,1249$, $1222,1018,887,732 \mathrm{~cm}^{-1}$.

\section{2-(2,2,2-trifluoroethoxy)benzo[d]thiazole $(4 e)^{[3]}$}

Yield: $84 \%$ (39 mg, colourless oil, $5 \%$ ethyl acetate in petroleum ether). ${ }^{1} \mathbf{H}-\mathbf{N M R}$ $\left(300 \mathrm{MHz}, \mathrm{CDCl}_{3}\right) \delta 7.76-7.66(\mathrm{~m}, 2 \mathrm{H}), 7.42(\mathrm{td}, J=7.8,1.3 \mathrm{~Hz}, 1 \mathrm{H}), 7.33-7.27$ $(\mathrm{m}, 1 \mathrm{H}), 4.97(\mathrm{q}, J=8.2 \mathrm{~Hz}, 2 \mathrm{H}) .{ }^{13} \mathrm{C}-\mathrm{NMR}\left(75 \mathrm{MHz}, \mathrm{CDCl}_{3}\right) \delta 171.0,148.3,132.3$,

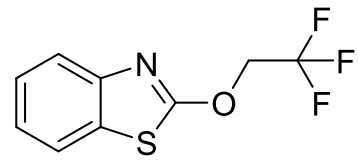
$126.3,124.3,121.5,121.3,66.8,66.3 .{ }^{19} \mathrm{~F}-\mathrm{NMR}\left(377 \mathrm{MHz}, \mathrm{CDCl}_{3}\right) \delta-73.80,-73.82,-73.84$.

\section{2-(cinnamyloxy)benzo[d]thiazole (4f)}

Yield: $42 \%$ (22 $\mathrm{mg}$, pale white solid, $5 \%$ ethyl acetate in petroleum ether). ${ }^{1} \mathrm{H}-\mathrm{NMR}\left(300 \mathrm{MHz}, \mathrm{CDCl}_{3}\right) \delta 7.70$ (ddd, $J=19.8,8.0,1.2 \mathrm{~Hz}, 2 \mathrm{H}$ ), $7.49-7.30(\mathrm{~m}, 6 \mathrm{H}), 7.29-7.22(\mathrm{~m}, 1 \mathrm{H}), 6.82(\mathrm{dt}, J=15.9,1.3 \mathrm{~Hz}, 1 \mathrm{H})$, $6.50(\mathrm{dt}, J=15.9,6.4 \mathrm{~Hz}, 1 \mathrm{H}), 5.24$ (dd, $J=6.5,1.3 \mathrm{~Hz}, 2 \mathrm{H}) .{ }^{13} \mathrm{C}-\mathrm{NMR}(75$

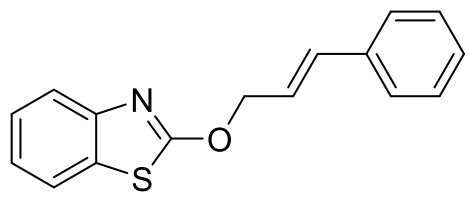
$\left.\mathrm{MHz}, \mathrm{CDCl}_{3}\right) \delta 172.6,149.3,136.0,135.2,132.0,128.7,128.3,126.8,126.0,123.5,122.5,121.3,120.8$, 
72.2. HRMS [EI]: $\mathrm{m} / \mathrm{z}$ calculated for $\mathrm{C}_{16} \mathrm{H}_{13} \mathrm{NOS}[\mathrm{M}]^{+} 267.0718$, found 267.0712. IR (ATR): ${ }^{v}=3028,1662$, $1531,1465,1246,1211,1184,929,694 \mathrm{~cm}^{-1}$.

\section{2-(but-2-yn-1-yloxy)benzo[d]thiazole (4g)}

Yield: $91 \%$ (37 $\mathrm{mg}$, colourless solid, 5\% ethyl acetate in petroleum ether).

${ }^{1} \mathrm{H}-\mathrm{NMR}\left(300 \mathrm{MHz}, \mathrm{CDCl}_{3}\right.$ ) $\delta 7.72$ (ddd, $J=8.1,1.3,0.6 \mathrm{~Hz}, 1 \mathrm{H}$ ), 7.65 (ddd, $J$

$=7.9,1.3,0.6 \mathrm{~Hz}, 1 \mathrm{H}), 7.38(\mathrm{td}, J=7.8,1.3 \mathrm{~Hz}, 1 \mathrm{H}), 7.28-7.20(\mathrm{~m}, 1 \mathrm{H})$,

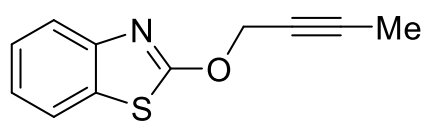

$5.16(\mathrm{q}, J=2.4 \mathrm{~Hz}, 2 \mathrm{H}), 1.91(\mathrm{t}, J=2.4 \mathrm{~Hz}, 3 \mathrm{H}) .{ }^{13} \mathrm{C}-\mathrm{NMR}\left(75 \mathrm{MHz}, \mathrm{CDCl}_{3}\right) \delta 171.9,149.1,132.1,126.0$, 123.7, 121.3, 121.0, 84.9, 72.7, 59.7, 3.8. HRMS [EI]: $\mathrm{m} / \mathrm{z}$ calculated for $\mathrm{C}_{11} \mathrm{H}_{9} \mathrm{NOS}[\mathrm{M}]^{+} 203.0405$, found 203.0400. IR (ATR): $v=2920,1735,1527,1438,1369,1238,1161,918,825,725 \mathrm{~cm}^{-1}$.

\section{2-(prop-2-yn-1-yloxy)benzo[d]thiazole (4h)}

Yield: $79 \%$ (30 mg, colourless solid, $5 \%$ ethyl acetate in petroleum ether). ${ }^{1} \mathbf{H}$ NMR $\left(300 \mathrm{MHz}, \mathrm{CDCl}_{3}\right) \delta 7.77-7.70(\mathrm{~m}, 1 \mathrm{H}), 7.70-7.62(\mathrm{~m}, 1 \mathrm{H}), 7.39(\mathrm{td}, \mathrm{J}=$ 7.8, $1.3 \mathrm{~Hz}, 1 \mathrm{H}), 7.26(\mathrm{td}, J=7.8,1.2 \mathrm{~Hz}, 1 \mathrm{H}), 5.19(\mathrm{~s}, 2 \mathrm{H}), 2.64(\mathrm{~s}, 1 \mathrm{H}) .{ }^{13} \mathrm{C}-\mathrm{NMR}$

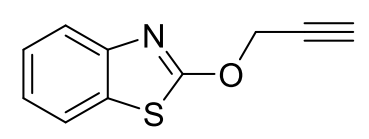
$\left(75 \mathrm{MHz}, \mathrm{CDCl}_{3}\right) \delta 171.6,148.9,132.2,126.1,123.8,121.4,121.1,77.2,76.3,58.6$. HRMS [EI]: $\mathrm{m} / \mathrm{z}$ calculated for $\mathrm{C}_{10} \mathrm{H}_{7} \mathrm{NOS}[\mathrm{M}]^{+} 189.0248$, found 189.0243. IR (ATR): $v=3267,2301,1739,1531,1435$, $1249,1211,918,763 \mathrm{~cm}^{-1}$.

\section{2-(1-phenylethoxy)benzo[d]thiazole (4i)}

Yield: $72 \%$ (37 mg, colourless glassy solid, 5\% ethyl acetate in petroleum ether). ${ }^{1} \mathrm{H}-\mathrm{NMR}\left(300 \mathrm{MHz}, \mathrm{CDCl}_{3}\right) \delta 7.75-7.60(\mathrm{~m}, 2 \mathrm{H}), 7.55-7.47(\mathrm{~m}, 2 \mathrm{H})$, $7.42-7.32(\mathrm{~m}, 4 \mathrm{H}), 7.22(\mathrm{td}, J=7.6,1.2 \mathrm{~Hz}, 1 \mathrm{H}), 6.32(\mathrm{q}, J=6.5 \mathrm{~Hz}, 1 \mathrm{H}), 1.80$ $(\mathrm{d}, J=6.5 \mathrm{~Hz}, 3 \mathrm{H}) .{ }^{13} \mathrm{C}-\mathrm{NMR}\left(75 \mathrm{MHz}, \mathrm{CDCl}_{3}\right) \delta 172.1,149.4,141.0,131.9$,

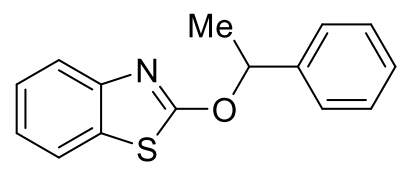
$128.6,128.2,126.3,125.9,123.4,121.2,120.8,80.1,22.4$. HRMS [EI]: $\mathrm{m} / \mathrm{z}$ calculated for $\mathrm{C}_{15} \mathrm{H}_{13} \mathrm{NOS}$ [M] ${ }^{+}$ 255.0718, found 255.0712. IR (ATR): $V=2981,1597,1527,1442,1246,1056,852,752 \mathrm{~cm}^{-1}$.

\section{2-(1-phenylpropoxy)benzo[d]thiazole (4j)}

Yield: $73 \%$ (39 $\mathrm{mg}$, pale white solid, 5\% ethyl acetate in petroleum ether). ${ }^{1}$ H-NMR (300 MHz, $\left.\mathrm{CDCl}_{3}\right) \delta 7.64$ (ddd, $J=14.2,8.0,1.2 \mathrm{~Hz}, 2 \mathrm{H}$ ), $7.52-7.45$ (m, 2H), $7.39-7.31(\mathrm{~m}, 4 \mathrm{H}), 7.20(\mathrm{td}, J=7.6,1.2 \mathrm{~Hz}, 1 \mathrm{H}), 6.09(\mathrm{t}, J=6.7 \mathrm{~Hz}$, $1 \mathrm{H}), 2.30-1.95(\mathrm{~m}, 2 \mathrm{H}), 1.02(\mathrm{t}, J=7.4 \mathrm{~Hz}, 3 \mathrm{H}) .{ }^{13} \mathrm{C}-\mathrm{NMR}\left(75 \mathrm{MHz}^{\mathrm{C}} \mathrm{CDCl}_{3}\right) \delta$

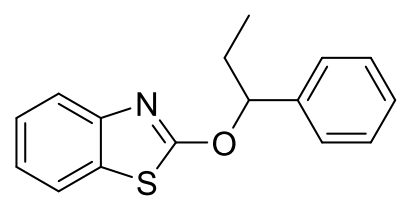
$172.4,149.4,139.8,131.9,128.4,128.1,126.7,125.8,123.3,121.1,120.8$, 85.1, 29.7, 9.9. HRMS [EI]: $\mathrm{m} / \mathrm{z}$ calculated for $\mathrm{C}_{16} \mathrm{H}_{15} \mathrm{NOS}[\mathrm{M}]^{+} 269.0874$, found 269.0870. IR (ATR): $v=$ 2970, 1597, 1527, 1442, 1207, 1064, 906, $752 \mathrm{~cm}^{-1}$. 


\section{2-(pent-1-yn-3-yloxy)benzo[d]thiazole (4k)}

Yield: $76 \%$ (33 mg, colourless liquid, $5 \%$ ethyl acetate in petroleum ether). ${ }^{1} \mathbf{H}$ NMR $\left(300 \mathrm{MHz}, \mathrm{CDCl}_{3}\right) \delta 7.78-7.70(\mathrm{~m}, 1 \mathrm{H}), 7.70-7.62(\mathrm{~m}, 1 \mathrm{H}), 7.43-7.35(\mathrm{~m}$, $1 \mathrm{H}), 7.29-7.20(\mathrm{~m}, 1 \mathrm{H}), 5.76(\mathrm{td}, J=6.4,2.1 \mathrm{~Hz}, 1 \mathrm{H}), 2.58(\mathrm{~d}, J=2.1 \mathrm{~Hz}, 1 \mathrm{H})$, $2.05(\mathrm{p}, J=7.4 \mathrm{~Hz}, 2 \mathrm{H}), 1.15(\mathrm{t}, J=7.4 \mathrm{~Hz}, 3 \mathrm{H}) .{ }^{13} \mathrm{C}-\mathrm{NMR}\left(75 \mathrm{MHz} \mathrm{CDCl}_{3}\right) \delta 171.7$,

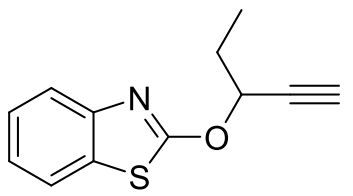
149.2, 132.1, 126.0, 123.6, 121.3, 121.1, 80.5, 75.0, 72.2, 28.1, 9.2. HRMS [EI]: $\mathrm{m} / \mathrm{z}$ calculated for $\mathrm{C}_{12} \mathrm{H}_{11} \mathrm{NOS}[\mathrm{M}]^{+} 217.0561$, found 217.0558. IR (ATR): $v=3294,2970,2360,1739,1527,1442,1211,902$, $752 \mathrm{~cm}^{-1}$.

\section{(1-(benzo[d]thiazol-2-yloxy)cyclohexyl)(phenyl)methanone (4I)}

Yield: $83 \%$ (56 $\mathrm{mg}$, pale white solid, $5 \%$ ethyl acetate in petroleum ether). ${ }^{1} \mathrm{H}-\mathrm{NMR}\left(400 \mathrm{MHz}, \mathrm{CDCl}_{3}\right) \delta 8.21-8.13(\mathrm{~m}, 2 \mathrm{H}), 7.56(\mathrm{dd}, J=7.9,1.2 \mathrm{~Hz}$, $1 \mathrm{H}), 7.50(\mathrm{dd}, J=8.1,1.1 \mathrm{~Hz}, 1 \mathrm{H}), 7.43-7.37(\mathrm{~m}, 1 \mathrm{H}), 7.36-7.30(\mathrm{~m}, 2 \mathrm{H})$,

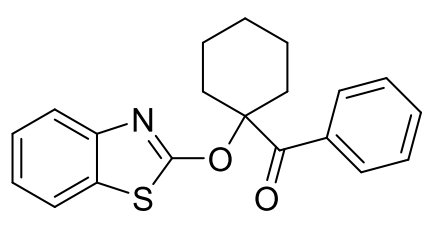
$7.26(\mathrm{td}, J=7.7,1.3 \mathrm{~Hz}, 1 \mathrm{H}), 7.15(\mathrm{td}, J=7.6,1.2 \mathrm{~Hz}, 1 \mathrm{H}), 2.81-2.65(\mathrm{~m}, 2 \mathrm{H}), 2.17-2.01(\mathrm{~m}, 2 \mathrm{H}), 1.68-$ $1.68(\mathrm{~m}, 5 \mathrm{H}), 1.51-1.34(\mathrm{~m}, 1 \mathrm{H}) .{ }^{13} \mathrm{C}-\mathrm{NMR}\left(101 \mathrm{MHz}, \mathrm{CDCl}_{3}\right) \delta$ 199.8, 169.3, 149.2, 135.2, 132.3, 132.0, 128.8, 128.2, 125.8, 123.4, 121.1, 121.0, 91.6, 32.6, 25.1, 21.3. HRMS [ESI]: $\mathrm{m} / \mathrm{z}$ calculated for $\mathrm{C}_{20} \mathrm{H}_{20} \mathrm{NO}_{2} \mathrm{~S}[\mathrm{M}+\mathrm{H}]^{+} 338.1215$, found 338.1209. IR (ATR): $v=2931,1735,1678,1523,1442,1134,921$, $752 \mathrm{~cm}^{-1}$.

\section{2-(((1R,2S,5R)-2-isopropyl-5-methylcyclohexyl)oxy)benzo[d]thiazole (4m)}

Yield: $42 \%$ (24 mg, colourless solid, $5 \%$ ethyl acetate in petroleum ether). ${ }^{1} \mathbf{H}$ NMR (300 MHz, CDCl $) 7.71-7.59(\mathrm{~m}, 2 \mathrm{H}), 7.36$ (td, $J=7.8,1.3 \mathrm{~Hz}, 1 \mathrm{H}), 7.21$ (td, $J$ $=7.8,1.2 \mathrm{~Hz}, 1 \mathrm{H}), 5.09(\mathrm{td}, J=10.8,4.5 \mathrm{~Hz}, 1 \mathrm{H}), 2.51-2.32(\mathrm{~m}, 1 \mathrm{H}), 2.17-1.98$ $(\mathrm{m}, 1 \mathrm{H}), 1.81-1.69(\mathrm{~m}, 2 \mathrm{H}), 1.67-1.51(\mathrm{~m}, 2 \mathrm{H}), 1.27-1.07(\mathrm{~m}, 3 \mathrm{H}), 0.96(\mathrm{~d}, J=$ $4.7 \mathrm{~Hz}, 3 \mathrm{H}), 0.94$ (d, $J=5.2 \mathrm{~Hz}, 3 \mathrm{H}), 0.84$ (d, $J=7.0 \mathrm{~Hz}, 3 \mathrm{H}) .{ }^{13} \mathrm{C}-\mathrm{NMR}(75 \mathrm{MHz}$,

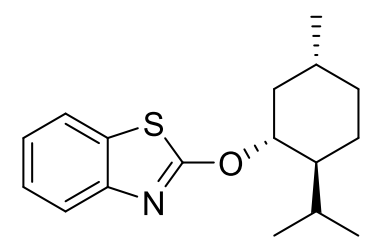
$\left.\mathrm{CDCl}_{3}\right) \delta 172.8,149.7,131.7,125.8,123.1,121.1,120.6,82.7,47.8,40.4,34.2,31.4,26.5,23.6,22.1$, 20.7, 16.7. HRMS [EI]: $\mathrm{m} / \mathrm{z}$ calculated for $\mathrm{C}_{17} \mathrm{H}_{23}$ NOS [M] ${ }^{+} 289.1500$, found 289.1501 . IR (ATR): ${ }^{V}=2951$, $1739,1527,1246,1215,948,752 \mathrm{~cm}^{-1}$.

2-(((3S,8R,9S,10R,13R,17R)-13-methyl-17-((R)-6-methylheptan-2-yl)-2,3,4,7,8,9,10,11,12,13,14,15, 16,17-tetradecahydro-1H-cyclopenta[a]phenanthren-3-yl)oxy)benzo[d]thiazole (4n)

Yield: $64 \%$ (67 mg, colourless solid, $10 \%$ ethyl acetate in petroleum ether). ${ }^{1} \mathrm{H}-\mathrm{NMR}\left(300 \mathrm{MHz}, \mathrm{CDCl}_{3}\right) 7.73-7.58$ $(\mathrm{m}, 2 \mathrm{H}), 7.36(\mathrm{td}, J=7.8,1.3 \mathrm{~Hz}, 1 \mathrm{H}), 7.21(\mathrm{td}, J=7.8,1.1$ $\mathrm{Hz}, 1 \mathrm{H}), 5.47(\mathrm{~d}, J=5.1 \mathrm{~Hz}, 1 \mathrm{H}), 5.04(\mathrm{td}, J=11.4,5.7 \mathrm{~Hz}$, $1 \mathrm{H}), 2.78-2.61(\mathrm{~m}, 1 \mathrm{H}), 2.60-2.42(\mathrm{~m}, 1 \mathrm{H}), 2.30-2.13$ $(m, 1 H), 2.07-1.73(m, 5 H), 1.62-1.00(m, 23 H), 0.95(d$, $J=6.5 \mathrm{~Hz}, 3 \mathrm{H}), 0.91(\mathrm{~d}, J=1.2 \mathrm{~Hz}, 3 \mathrm{H}), 0.89(\mathrm{~d}, J=1.2 \mathrm{~Hz}$, 3H), 0.71 (s, 3H). ${ }^{13} \mathrm{C}-\mathrm{NMR}\left(75 \mathrm{MHz}, \mathrm{CDCl}_{3}\right) \delta$ 172.2, 149.7,

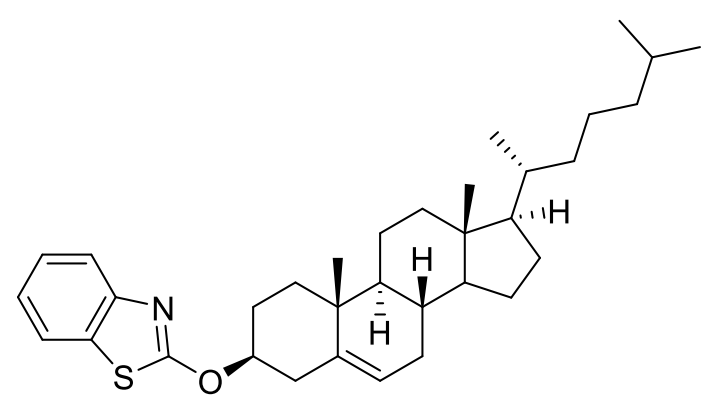


139.4, 131.8, 125.8, 123.2, 123.1, 121.1, 120.7, 81.8, 56.7, 56.2, 50.1, 42.3, 39.8, 39.6, 38.2, 37.0, 36.7, $36.2,35.8,32.0,31.9,28.3,28.1,27.8,24.3,23.9,22.9,22.6,21.1,19.4,18.8,11.9$. HRMS [EI]: $\mathrm{m} / \mathrm{z}$ calculated for $\mathrm{C}_{34} \mathrm{H}_{49} \mathrm{NOS}[\mathrm{M}]^{+} 519.3535$, found 519.3544. IR (ATR): $V=2947,1739,1527,1442,1365$, $1246,1215,732 \mathrm{~cm}^{-1}$.

\section{2-phenoxybenzo[d]thiazole $(40)^{[4]}$}

Yield: $82 \%$ (37 mg, colourless solid, $5 \%$ ethyl acetate in petroleum ether). ${ }^{1} \mathrm{H}$ NMR $\left(300 \mathrm{MHz}, \mathrm{CDCl}_{3}\right) \delta 7.81-7.74(\mathrm{~m}, 1 \mathrm{H}), 7.73-7.65(\mathrm{~m}, 1 \mathrm{H}), 7.53-7.36(\mathrm{~m}$, $5 \mathrm{H}), 7.36-7.25(\mathrm{~m}, 2 \mathrm{H}) .{ }^{13} \mathrm{C}-\mathrm{NMR}\left(75 \mathrm{MHz}, \mathrm{CDCl}_{3}\right) \delta 172.0,154.8,149.1,132.3$,

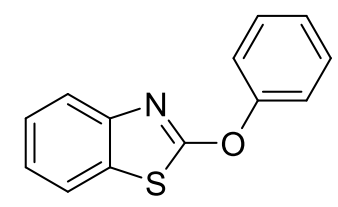
$130.0,126.3,126.3,124.1,121.8,121.3,120.7$.

\section{2-(4-(tert-butyl)phenoxy)benzo[d]thiazole $(4 p)^{[5]}$}

Yield: $90 \%$ (51 mg, colourless oil, $5 \%$ ethyl acetate in petroleum ether). ${ }^{1} \mathbf{H}$ NMR $\left(300 \mathrm{MHz}, \mathrm{CDCl}_{3}\right) \delta 7.80-7.72(\mathrm{~m}, 1 \mathrm{H}), 7.70-7.65(\mathrm{~m}, 1 \mathrm{H}), 7.51-7.43$ (m, 2H), 7.40 (td, J = 7.8, $1.3 \mathrm{~Hz}, 1 \mathrm{H}), 7.32-7.25(\mathrm{~m}, 3 \mathrm{H}), 1.37(\mathrm{~s}, 9 \mathrm{H}) .{ }^{13} \mathrm{C}-\mathrm{NMR}$ $\left(75 \mathrm{MHz}, \mathrm{CDCl}_{3}\right) \delta 172.3,152.5,149.2,149.2,132.3,126.9,126.2,123.9,121.7$, $121.2,120.0,34.6,31.4$.

\section{2-(4-methoxyphenoxy)benzo[d]thiazole $(4 q)^{[5]}$}

Yield: $99 \%$ (51 mg, colourless oil, $10 \%$ ethyl acetate in petroleum ether). ${ }^{1} \mathbf{H}$ NMR $\left(300 \mathrm{MHz}, \mathrm{CDCl}_{3}\right) \delta 7.65$ (ddd, $J=8.1,1.3,0.6 \mathrm{~Hz}, 1 \mathrm{H}$ ), 7.56 (ddd, $J=7.9$, $1.3,0.6 \mathrm{~Hz}, 1 \mathrm{H}$ ), 7.30 (ddd, J = 8.1, 7.4, $1.3 \mathrm{~Hz}, 1 \mathrm{H}$ ), $7.23-7.13(\mathrm{~m}, 3 \mathrm{H}), 6.92-$ $6.82(\mathrm{~m}, 2 \mathrm{H}), 3.75(\mathrm{~s}, 3 \mathrm{H}) .{ }^{13} \mathrm{C}$ NMR $\left(75 \mathrm{MHz}, \mathrm{CDCl}_{3}\right) \delta$ 172.9, 157.7, 149.2, $148.4,132.2,126.2,123.9,121.9,121.7,121.3,114.9,55.7$.

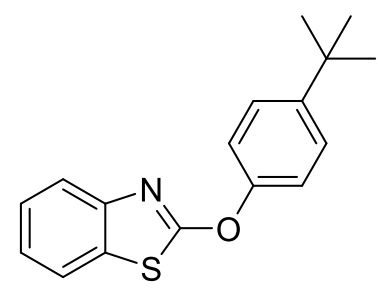

\section{2-(p-tolyloxy)benzo[d]thiazole (4r)}

Yield: $62 \%$ (30 mg, colourless oil, 5\% ethyl acetate in petroleum ether). ${ }^{1} \mathbf{H}$ NMR $\left(300 \mathrm{MHz}, \mathrm{CDCl}_{3}\right.$ ) $\delta 7.76$ (ddd, $J=8.1,1.3,0.6 \mathrm{~Hz}, 1 \mathrm{H}$ ), 7.67 (ddd, $J=7.9,1.3,0.6$ $\mathrm{Hz}, 1 \mathrm{H}$ ), 7.40 (ddd, $J=8.1,7.3,1.4 \mathrm{~Hz}, 1 \mathrm{H}), 7.33-7.21(\mathrm{~m}, 5 \mathrm{H}), 2.41(\mathrm{~s}, 3 \mathrm{H}) .{ }^{13} \mathrm{C}$ NMR $\left(75 \mathrm{MHz}_{1} \mathrm{CDCl}_{3}\right) \delta 172.5,152.6,149.2,136.2,132.3,130.5,126.2,123.9$, 121.7, 121.3, 120.5, 21.0. HRMS [EI]: $\mathrm{m} / \mathrm{z}$ calculated for $\mathrm{C}_{14} \mathrm{H}_{11} \mathrm{NOS}$ [M] ${ }^{+}$<smiles>COc1ccc(Oc2nc3ccccc3s2)cc1</smiles>
241.0561, found 241.0556. IR (ATR): 3062, 1739, 1527, 1496, 1438, 1119, 1161, 817, $759 \mathrm{~cm}^{-1}$.

\section{2-(m-tolyloxy)benzo[d]thiazole (4s)}

Yield: $60 \%$ (28 mg, colourless oil, $5 \%$ ethyl acetate in petroleum ether). ${ }^{1} \mathbf{H}$ NMR $\left(400 \mathrm{MHz}, \mathrm{CDCl}_{3}\right) \delta 7.76(\mathrm{dd}, J=8.2,1.1 \mathrm{~Hz}, 1 \mathrm{H}), 7.68(\mathrm{dd}, J=7.8,1.4$ $\mathrm{Hz}, 1 \mathrm{H}), 7.41(\mathrm{td}, J=7.8,1.3 \mathrm{~Hz}, 1 \mathrm{H}), 7.37-7.25(\mathrm{~m}, 5 \mathrm{H}), 2.34(\mathrm{~s}, 3 \mathrm{H}) .{ }^{13} \mathrm{C}$ NMR $\left(101 \mathrm{MHz}, \mathrm{CDCl}_{3}\right) \delta 172.3,153.3,149.3,132.3,131.8,130.4,127.5$,

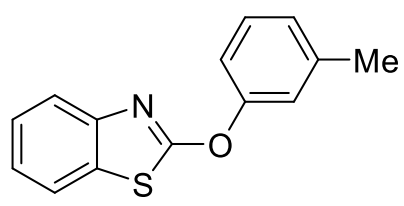


126.8, 126.3, 123.9, 121.7, 121.3, 121.3, 16.2. HRMS [EI]: $\mathrm{m} / \mathrm{z}$ calculated for $\mathrm{C}_{14} \mathrm{H}_{11} \mathrm{NOS}[\mathrm{M}]^{+} 241.0552$, found 241.0561. IR (ATR): 3055, 1739, 1523, 1442, 1165, 1103, 798, $759 \mathrm{~cm}^{-1}$.

\section{2-(o-tolyloxy)benzo[d]thiazole (4t)}

Yield: $70 \%$ (34 mg, colourless oil, ethyl acetate in petroleum ether). ${ }^{1} \mathbf{H}$ NMR $\left(300 \mathrm{MHz}, \mathrm{CDCl}_{3}\right) \delta 7.80-7.74(\mathrm{~m}, 1 \mathrm{H}), 7.73-7.64(\mathrm{~m}, 1 \mathrm{H}), 7.44-7.37(\mathrm{~m}, 1 \mathrm{H})$, $7.36-7.23(\mathrm{~m}, 2 \mathrm{H}), 7.22-7.10(\mathrm{~m}, 3 \mathrm{H}), 2.42(\mathrm{~s}, 3 \mathrm{H}) .{ }^{13} \mathrm{C} \mathrm{NMR}\left(75 \mathrm{MHz}^{\mathrm{C}} \mathrm{CDCl}_{3}\right) \delta$ $172.2,154.8,149.1,140.3,132.3,129.7,127.2,126.2,124.0,121.7,121.3$,

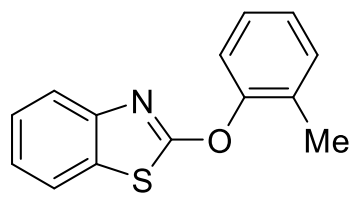
121.2, 117.6, 21.4. HRMS [EI]: $\mathrm{m} / \mathrm{z}$ calculated for $\mathrm{C}_{14} \mathrm{H}_{11} \mathrm{NOS}$ [M] ${ }^{+}$241.0561, found 241.0557. IR (ATR): 2920, 1732, 1516, 1438, 1207, 1130, 914, $756 \mathrm{~cm}^{-1}$.

\section{1,4-bis(benzo[d]thiazol-2-yloxy)benzene (4u)}

Yield: $75 \%$ (36 mg, colourless solid, 5\% MeOH in DCM). ${ }^{1} \mathbf{H}$ NMR (600 MHz, DMSO- $\left.d_{6}\right) \delta 9.71(\mathrm{~s}, 1 \mathrm{H}), 7.90(\mathrm{dd}, J=8.1,1.2 \mathrm{~Hz}, 1 \mathrm{H}), 7.68(\mathrm{dd}, J=8.2,1.2 \mathrm{~Hz}$, $1 \mathrm{H}), 7.42$ (ddd, $J=8.3,7.4,1.3 \mathrm{~Hz}, 1 \mathrm{H}$ ), $7.31(\mathrm{td}, J=7.7,1.2 \mathrm{~Hz}, 1 \mathrm{H}), 7.28-7.23$ $(\mathrm{m}, 2 \mathrm{H}), 6.88-6.84(\mathrm{~m}, 2 \mathrm{H}) .{ }^{13} \mathrm{C}$ NMR $\left(151 \mathrm{MHz}\right.$, DMSO- $\left.d_{6}\right) \delta 173.5,156.4$, 149.3, 147.3, 132.2, 126.9, 124.4, 122.6, 122.5, 121.4, 116.7. HRMS [EI]: $\mathrm{m} / \mathrm{z}$

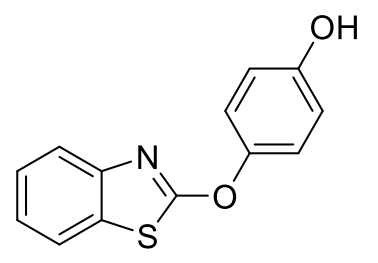
calculated for $\mathrm{C}_{13} \mathrm{H}_{9} \mathrm{NO}_{2} \mathrm{~S}[\mathrm{M}+\mathrm{H}]^{+}$244.0432, found 244.0424. IR (ATR): 3098, 2924, 2800, 2681, 2596, $1601,1524,1497,1443,1369,1231,1180,918,829,760 \mathrm{~cm}^{-1}$.

\section{7-(benzo[d]thiazol-2-yloxy)-4-methyl-2H-chromen-2-one (4v)}

Yield: $59 \%$ (36 mg, pale white solid, 30\% ethyl acetate in petroleum ether). ${ }^{1} \mathrm{H}-\mathrm{NMR}\left(300 \mathrm{MHz}, \mathrm{CDCl}_{3}\right) \delta 7.80-7.71(\mathrm{~m}, 2 \mathrm{H}), 7.68(\mathrm{~d}, J=8.7 \mathrm{~Hz}$, $1 \mathrm{H}), 7.49(\mathrm{~d}, J=2.4 \mathrm{~Hz}, 1 \mathrm{H}), 7.43(\mathrm{td}, J=7.7,1.4 \mathrm{~Hz}, 1 \mathrm{H}), 7.40-7.28(\mathrm{~m}$, $2 \mathrm{H}), 6.30(\mathrm{q}, J=1.3 \mathrm{~Hz}, 1 \mathrm{H}), 2.47(\mathrm{~d}, J=1.3 \mathrm{~Hz}, 3 \mathrm{H}) .{ }^{13} \mathrm{C}-\mathrm{NMR}(75 \mathrm{MHz}$, $\left.\mathrm{CDCl}_{3}\right) \delta 170.2,160.4,156.6,154.4,151.9,148.6,132.3,126.5,125.9$, 124.6, 122.0, 121.4, 117.8, 116.5, 114.5, 109.0, 18.8. HRMS [EI]: m/z

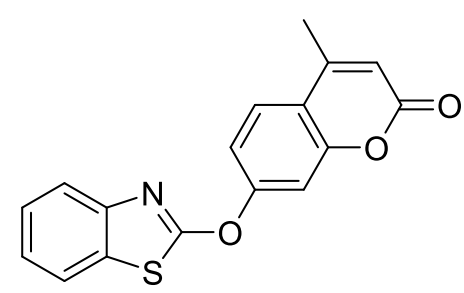
calculated for $\mathrm{C}_{17} \mathrm{H}_{11} \mathrm{NO}_{3} \mathrm{~S}[\mathrm{M}]^{+} 309.0460$, found 309.0459. IR (ATR): $V=2954,1716,1612,1523,1438$, $1365,1219,1014,910,860,756 \mathrm{~cm}^{-1}$.

\section{2-(naphthalen-1-yloxy)benzo[d]thiazole $(4 w)^{[5]}$}

Yield: $76 \%$ (42 mg, pale white solid, $5 \%$ ethyl acetate in petroleum ether). ${ }^{1} \mathbf{H}$ NMR $\left(300 \mathrm{MHz}, \mathrm{CDCl}_{3}\right) \delta 8.17-8.08(\mathrm{~m}, 1 \mathrm{H}), 7.97-7.90(\mathrm{~m}, 1 \mathrm{H}), 7.88-7.82$ (m, 1H), $7.81-7.74(\mathrm{~m}, 1 \mathrm{H}), 7.66(\mathrm{dd}, J=8.0,1.3 \mathrm{~Hz}, 1 \mathrm{H}), 7.62-7.48(\mathrm{~m}$, $4 \mathrm{H}), 7.41$ (ddd, $J=8.2,7.4,1.3 \mathrm{~Hz}, 1 \mathrm{H}), 7.34-7.24(\mathrm{~m}, 1 \mathrm{H}) .{ }^{13} \mathrm{C}$ NMR $(75$

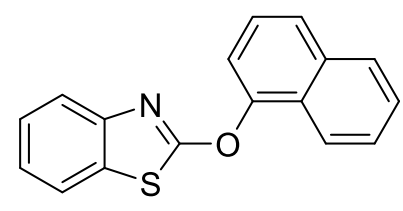
$\left.\mathrm{MHz}, \mathrm{CDCl}_{3}\right) \delta 172.7,150.8,149.1,135.0,132.3,128.1,126.9,126.9,126.8,126.5,126.3,125.5,124.1$, $121.8,121.4,121.3,117.2$. 


\section{2-(4-bromophenoxy)benzo[d]thiazole (4x)}

Yield: $83 \%$ (50 mg, pale white solid, $5 \%$ ethyl acetate in petroleum ether). ${ }^{1} \mathrm{H}$ NMR $\left(300 \mathrm{MHz}, \mathrm{CDCl}_{3}\right) \delta 7.80-7.66(\mathrm{~m}, 2 \mathrm{H}), 7.62-7.53(\mathrm{~m}, 2 \mathrm{H}), 7.41(\mathrm{td}, J=$ 7.8, $1.3 \mathrm{~Hz}, 1 \mathrm{H}), 7.35-7.24(\mathrm{~m}, 3 \mathrm{H}) .{ }^{13} \mathrm{C}-\mathrm{NMR}\left(75 \mathrm{MHz}, \mathrm{CDCl}_{3}\right) \delta 171.2,153.6$, $148.9,132.9,132.3,126.4,124.3,122.4,121.8,121.4,119.2$. HRMS [EI]: $\mathrm{m} / \mathrm{z}$ calculated for $\mathrm{C}_{13} \mathrm{H}_{8} \mathrm{BrNOS}[\mathrm{M}]^{+} 304.9510$, found 304.9505. IR (ATR): $v=2920$,

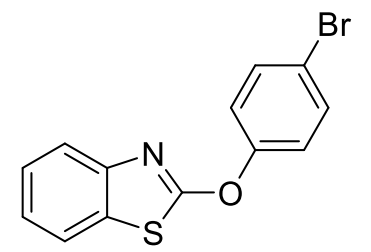
2364, 1739, 1519, 1438, 1230, 1064, 813, $717 \mathrm{~cm}^{-1}$.

\section{2-(3-bromophenoxy)benzo[d]thiazole (4y)}

Yield: $87 \%$ (53 mg, colourless solid, $5 \%$ ethyl acetate in petroleum ether). ${ }^{1} \mathbf{H}$ NMR $\left(300 \mathrm{MHz}, \mathrm{CDCl}_{3}\right) \delta 7.77(\mathrm{dd}, J=8.1,1.2 \mathrm{~Hz}, 1 \mathrm{H}), 7.71(\mathrm{dd}, J=7.9,1.3 \mathrm{~Hz}$, $1 \mathrm{H}), 7.61-7.57(\mathrm{~m}, 1 \mathrm{H}), 7.49-7.38(\mathrm{~m}, 2 \mathrm{H}), 7.38-7.29(\mathrm{~m}, 3 \mathrm{H}) .{ }^{13} \mathrm{C}$ NMR

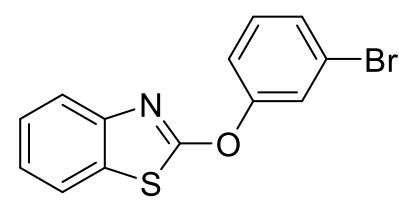
$\left(75 \mathrm{MHz}, \mathrm{CDCl}_{3}\right) \delta 171.0,154.9,148.8,132.3,130.9,129.3,126.4,124.4,124.0,122.8,121.9,121.4$, 119.3. HRMS [EI]: $\mathrm{m} / \mathrm{z}$ calculated for $\mathrm{C}_{13} \mathrm{H}_{8} \mathrm{BrNOS}$ [M] ${ }^{+} 304.9510$, found 304.9502. IR (ATR): ${ }^{V}=2924$, $1735,1519,1207,1056,852,756 \mathrm{~cm}^{-1}$.

\section{2-(2-bromophenoxy)benzo[d]thiazole (4z)}

Yield: $68 \%$ (41 mg, colourless solid, 5\% ethyl acetate in petroleum ether). ${ }^{1} \mathrm{H}$ NMR $\left(300 \mathrm{MHz}, \mathrm{CDCl}_{3}\right) \delta 7.81-7.64(\mathrm{~m}, 3 \mathrm{H}), 7.51-7.36(\mathrm{~m}, 3 \mathrm{H}), 7.33-7.19(\mathrm{~m}$, 2H). ${ }^{13} \mathrm{C}-\mathrm{NMR}\left(75 \mathrm{MHz}, \mathrm{CDCl}_{3}\right) \delta 171.2,151.7,149.0,134.0,132.5,128.9,127.8$,

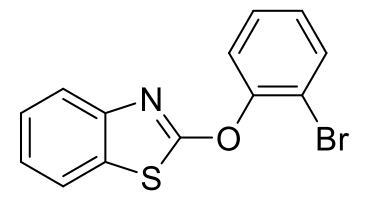
126.3, 124.2, 123.2, 121.9, 121.4, 115.7. HRMS [EI]: $\mathrm{m} / \mathrm{z}$ calculated for $\mathrm{C}_{13} \mathrm{H}_{8} \mathrm{BrNOS}[\mathrm{M}]^{+} 304.9510$, found 304.9508. IR (ATR): $V=3055,2360,1739,1523,1465,1438,1203,1014,744 \mathrm{~cm}^{-1}$.

\section{2-(2,4-difluorophenoxy)benzo[d]thiazole (4aa)}

Yield: $59 \%$ (31 mg, colourless solid, $5 \%$ ethyl acetate in petroleum ether). ${ }^{1} \mathbf{H}$ NMR (300 MHz, $\left.\mathrm{CDCl}_{3}\right) \delta 7.75-7.67(\mathrm{~m}, 2 \mathrm{H}), 7.50-7.36(\mathrm{~m}, 2 \mathrm{H}), 7.34-7.27(\mathrm{~m}$, $1 \mathrm{H}), 7.08-6.91(\mathrm{~m}, 2 \mathrm{H}) .{ }^{13} \mathrm{C}-\mathrm{NMR}\left(75 \mathrm{MHz}, \mathrm{CDCl}_{3}\right) \delta 171.2,162.0,161.9,158.7$, $158.6,155.8,155.6,152.4,152.3,148.7,138.2,138.0,138.0,132.5,126.4,124.3$, $124.0,123.9,121.8,121.4,111.8,111.7,111.5,111.4,106.0,105.7,105.7,105.4$. HRMS [EI]: $\mathrm{m} / \mathrm{z}$ calculated for $\mathrm{C}_{13} \mathrm{H}_{7} \mathrm{~F}_{2} \mathrm{NOS}$ [M] ${ }^{+}$263.0216, found 263.0210. IR

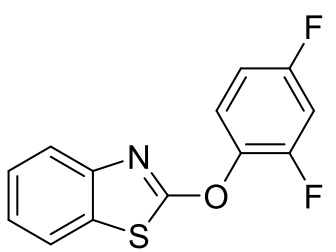
(ATR): $v=2920,2360,1739,1616,1527,1496,1438,1303,1176,1091,960,852,752 \mathrm{~cm}^{-1}$.

\section{2-(4-bromo-2-chlorophenoxy)benzo[d]thiazole (4ab)}

Yield: $60 \%$ (40 mg, pale white solid, $5 \%$ ethyl acetate in petroleum ether). ${ }^{1} \mathbf{H}$ NMR $\left(300 \mathrm{MHz}, \mathrm{CDCl}_{3}\right) \delta 7.75-7.69(\mathrm{~m}, 2 \mathrm{H}), 7.68(\mathrm{~d}, J=2.3 \mathrm{~Hz}, 1 \mathrm{H}), 7.50$ (dd, $J=$ 8.7, $2.3 \mathrm{~Hz}, 1 \mathrm{H}), 7.44-7.28$ (m, 3H). ${ }^{13} \mathrm{C}-\mathrm{NMR}\left(75 \mathrm{MHz}, \mathrm{CDCl}_{3}\right) \delta 170.6,149.6$, $148.7,133.5,132.5,131.3,127.9,126.4,124.4,124.4,121.9,121.4,119.6$. HRMS [EI]: $\mathrm{m} / \mathrm{z}$ calculated for $\mathrm{C}_{13} \mathrm{H}_{7} \mathrm{BrClNOS}$ [M] 338.9120 , found 338.9118. IR

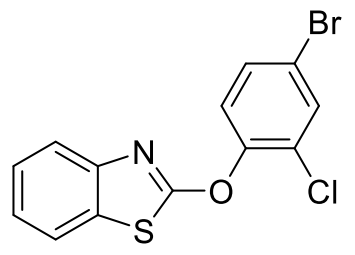


(ATR): ${ }^{V}=2924,1735,1527,1469,1438,1257,1056,821,748 \mathrm{~cm}^{-1}$.

\section{4-(benzo[d]thiazol-2-yloxy)benzonitrile (4ac)}

Yield: $66 \%$ (33 mg, colourless solid, $5 \%$ ethyl acetate in petroleum ether). ${ }^{1} \mathbf{H}$ NMR $\left(300 \mathrm{MHz}, \mathrm{CDCl}_{3}\right) \delta 7.83-7.69(\mathrm{~m}, 4 \mathrm{H}), 7.60-7.53(\mathrm{~m}, 2 \mathrm{H}), 7.44(\mathrm{td}, J=$ 7.7, $1.3 \mathrm{~Hz}, 1 \mathrm{H}), 7.34$ (td, $J=7.7,1.2 \mathrm{~Hz}, 1 \mathrm{H}) .{ }^{13} \mathrm{C}$-NMR $\left(75 \mathrm{MHz}, \mathrm{CDCl}_{3}\right) \delta 169.8$, $157.5,148.5,134.1,132.3,126.6,124.8,122.0,121.5,121.1,118.2,109.5$. HRMS [EI]: $\mathrm{m} / \mathrm{z}$ calculated for $\mathrm{C}_{14} \mathrm{H}_{8} \mathrm{~N}_{2} \mathrm{OS}$ [M] 252.0357 , found 252.0352. IR

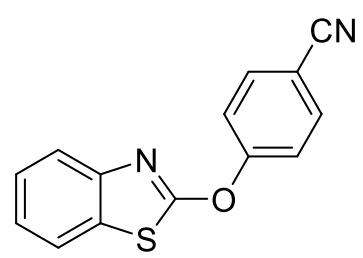
(ATR): $v=2924,2225,1739,1593,1523,1496,1435,1203,833,756 \mathrm{~cm}^{-1}$.

\section{$\mathrm{N}, \mathrm{N}$-diphenylbenzo[d]thiazol-2-amine (4ad) ${ }^{[6]}$}

Yield: $81 \%$ (49 mg, brown solid, 5\% ethyl acetate in petroleum ether). ${ }^{1} \mathbf{H}-\mathbf{N M R}$ $\left(400 \mathrm{MHz}, \mathrm{CDCl}_{3}\right) \delta 7.73-7.67(\mathrm{~m}, 1 \mathrm{H}), 7.60-7.55(\mathrm{~m}, 1 \mathrm{H}), 7.52-7.39(\mathrm{~m}, 8 \mathrm{H})$, $7.38-7.28(\mathrm{~m}, 3 \mathrm{H}), 7.16(\mathrm{td}, J=7.6,1.2 \mathrm{~Hz}, 1 \mathrm{H}) .{ }^{13} \mathrm{C}-\mathrm{NMR}\left(101 \mathrm{MHz}, \mathrm{CDCl}_{3}\right) \delta$ $167.3,152.3,144.7,131.9,129.7,126.5,126.5,125.9,122.6,120.4,120.4$.

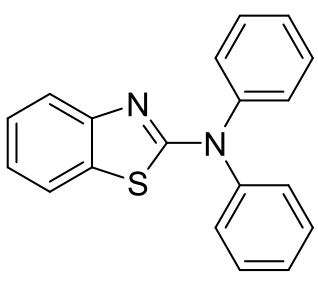

\section{$\mathrm{N}$-methyl-N-phenylbenzo[d]thiazol-2-amine $(4 \mathrm{ae})^{[6]}$}

Yield: $61 \%$ (29 mg, pale white solid, $5 \%$ ethyl acetate in petroleum ether). ${ }^{1} \mathbf{H}$ NMR $\left(400 \mathrm{MHz}, \mathrm{CDCl}_{3}\right) \delta 7.67-7.61(\mathrm{~m}, 1 \mathrm{H}), 7.53-7.41(\mathrm{~m}, 5 \mathrm{H}), 7.39-7.28(\mathrm{~m}$, $2 \mathrm{H}), 7.09$ (td, $J=7.6,1.2 \mathrm{~Hz}, 1 \mathrm{H}), 3.66(\mathrm{~s}, 3 \mathrm{H}) .{ }^{13} \mathrm{C}-\mathrm{NMR}\left(101 \mathrm{MHz}, \mathrm{CDCl}_{3}\right) \delta 168.3$, $152.7,145.8,131.2,129.9,127.4,126.0,125.9,121.7,120.5,119.2,40.5$.

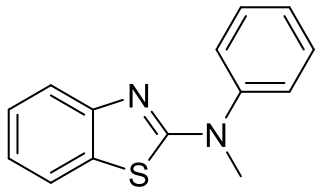

\section{$\mathrm{N}$-methyl-N-(pyridin-2-yl)benzo[d]thiazol-2-amine (4af)}

Yield: $75 \%$ (36 mg, pale white solid, $10 \%$ ethyl acetate in petroleum ether). ${ }^{1} \mathbf{H}$ NMR $\left(400 \mathrm{MHz}, \mathrm{CDCl}_{3}\right) \delta 8.49$ (ddd, $\left.J=4.8,1.8,0.7 \mathrm{~Hz}, 1 \mathrm{H}\right), 7.84-7.69(\mathrm{~m}, 3 \mathrm{H})$, 7.41 (ddd, $J=8.2,7.2,1.3 \mathrm{~Hz}, 1 \mathrm{H}), 7.24$ (td, $J=7.5,1.1 \mathrm{~Hz}, 1 \mathrm{H}), 7.19-7.12(\mathrm{~m}, 1 \mathrm{H})$, 7.03 (ddd, $J=7.3,5.0,0.8 \mathrm{~Hz}, 1 \mathrm{H}$ ), $3.93(\mathrm{~s}, 3 \mathrm{H}) .{ }^{13} \mathrm{C}-\mathrm{NMR}\left(101 \mathrm{MHz}, \mathrm{CDCl}_{3}\right) \delta$ 162.0,

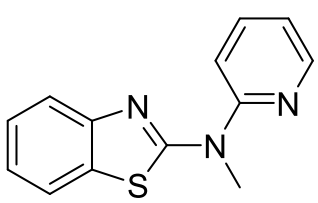
153.7, 149.2, 146.1, 138.3, 133.2, 125.6, 122.5, 120.5, 120.0, 117.4, 110.1, 36.0. HRMS [EI]: m/z calculated for $\mathrm{C}_{13} \mathrm{H}_{11} \mathrm{~N}_{3} \mathrm{~S}[\mathrm{M}]^{+} 241.0674$, found 241.0669. IR (ATR): $v=2924,2326,1739,1496,1469$, $1334,825,748 \mathrm{~cm}^{-1}$.

\section{N-phenylbenzo[d]thiazol-2-amine (4ag) ${ }^{[7]}$}

Yield: $30 \%$ (13 mg, colourless needles, $20 \%$ ethyl acetate in petroleum ether). ${ }^{1} \mathrm{H}$ NMR $\left(300 \mathrm{MHz}, \mathrm{CDCl}_{3}\right) \delta 7.65(\mathrm{~d}, J=7.8 \mathrm{~Hz}, 1 \mathrm{H}), 7.59(\mathrm{~d}, J=8.0 \mathrm{~Hz}, 1 \mathrm{H}), 7.56-$ $7.48(\mathrm{~m}, 2 \mathrm{H}), 7.47-7.38(\mathrm{~m}, 2 \mathrm{H}), 7.38-7.30(\mathrm{~m}, 1 \mathrm{H}), 7.25-7.12(\mathrm{~m}, 2 \mathrm{H}) .{ }^{13} \mathrm{C}-$<smiles>c1ccc(Nc2nc3ccccc3s2)cc1</smiles>
NMR $\left(75 \mathrm{MHz}, \mathrm{CDCl}_{3}\right) \delta 164.8,151.3,139.9,129.8,129.6,126.2,124.4,122.4,120.9,120.3,119.3$. 


\section{$\mathrm{N}$-(benzo[d]thiazol-2-yl)-N-methylbenzamide (4ah)}

Yield: $26 \%$ (14 mg, whith solid, $50 \%$ ethyl acetate in petroleum ether). ${ }^{1} \mathbf{H}$ NMR (300 MHz, $\left.\mathrm{CDCl}_{3}\right) \delta 7.95-7.80(\mathrm{~m}, 2 \mathrm{H}), 7.65-7.57(\mathrm{~m}, 2 \mathrm{H}), 7.57-7.42$ $(\mathrm{m}, 4 \mathrm{H}), 7.34(\mathrm{td}, J=7.7,1.1 \mathrm{~Hz}, 1 \mathrm{H}), 3.77(\mathrm{~s}, 3 \mathrm{H}) .{ }^{13} \mathrm{C}-\mathrm{NMR}\left(75 \mathrm{MHz}, \mathrm{CDCl}_{3}\right) \delta$ $171.1,160.3,148.3,134.4,133.3,131.2,128.7,127.6,126.0,124.1,121.5$,

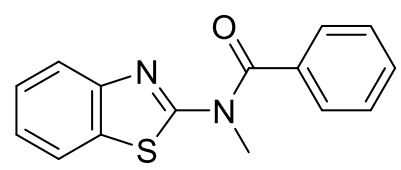
121.2, 38.8. HRMS [EI]: $\mathrm{m} / \mathrm{z}$ calculated for $\mathrm{C}_{15} \mathrm{H}_{12} \mathrm{~N}_{2} \mathrm{OS}$ [M] 268.0670 , found 268.06644. IR (ATR): $v=$ $2920,1647,1489,1423,1350,1276,1246,1010,756 \mathrm{~cm}^{-1}$.

\section{$\mathrm{N}$-methylbenzo[d]thiazol-2-amine (4ai) ${ }^{[8]}$}

Yield: $55 \%$ (18 mg, colourless needles, $50 \%$ ethyl acetate in petroleum ether). ${ }^{1} \mathbf{H}$ $\operatorname{NMR}\left(300 \mathrm{MHz}, \mathrm{CDCl}_{3}\right) \delta 7.67-7.57(\mathrm{~m}, 1 \mathrm{H}), 7.57-7.48(\mathrm{~m}, 1 \mathrm{H}), 7.31$ (td, $J=7.8,1.3$ $\mathrm{Hz}, 1 \mathrm{H}), 7.09$ (td, $J=7.8,1.1 \mathrm{~Hz}, 1 \mathrm{H}), 6.52(\mathrm{~s}, 1 \mathrm{H}), 3.11(\mathrm{~s}, 3 \mathrm{H}) .{ }^{13} \mathrm{C}-\mathrm{NMR}(75 \mathrm{MHz}$,<smiles>CNc1nc2ccccc2s1</smiles>
$\left.\mathrm{CDCl}_{3}\right) \delta 169.0,152.5,130.3,126.0,121.3,120.9,118.6,31.7$.

\section{2-(1H-pyrrol-1-yl)benzo[d]thiazole (4aj) ${ }^{[9]}$}

Yield: $75 \%$ (30 mg, pale white solid, $5 \%$ ethyl acetate in petroleum ether). ${ }^{1} \mathbf{H}$ NMR (300 MHz, CDCl $) \delta 7.93-7.86(\mathrm{~m}, 1 \mathrm{H}), 7.84-7.73(\mathrm{~m}, 1 \mathrm{H}), 7.54-7.41(\mathrm{~m}$, 3H), 7.34 (ddd, $J=8.5,7.3,1.2 \mathrm{~Hz}, 1 \mathrm{H}), 6.45-6.35(\mathrm{~m}, 2 \mathrm{H}) .{ }^{13} \mathrm{C}-\mathrm{NMR}(75 \mathrm{MHz}$,

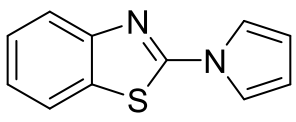
$\left.\mathrm{CDCl}_{3}\right) \delta 159.5,151.1,132.0,126.7,124.5,122.1,121.3,120.1,112.7$.

\section{2-(2,4-dimethyl-1H-pyrrol-1-yl)benzo[d]thiazole (4ak)}

Yield: $63 \%$ (28 mg, yellow glassy solid, $5 \%$ ethyl acetate in petroleum ether). ${ }^{1} \mathbf{H}$ NMR $\left(300 \mathrm{MHz}, \mathrm{CDCl}_{3}\right) \delta 7.92-7.85(\mathrm{~m}, 1 \mathrm{H}), 7.83-7.75(\mathrm{~m}, 1 \mathrm{H}), 7.46(\mathrm{td}, J=8.2$,<smiles>Cc1cc(C)n(-c2nc3ccccc3s2)c1</smiles>
7.8, 1.2 Hz, 1H), $7.36-7.31(\mathrm{~m}, 1 \mathrm{H}), 7.06(\mathrm{~s}, 1 \mathrm{H}), 5.95(\mathrm{~s}, 1 \mathrm{H}), 2.62(\mathrm{~d}, J=1.0 \mathrm{~Hz}$, 3H), $2.11(\mathrm{~d}, J=1.1 \mathrm{~Hz}, 3 \mathrm{H}) .{ }^{13} \mathrm{C}-\mathrm{NMR}\left(75 \mathrm{MHz}, \mathrm{CDCl}_{3}\right) \delta 159.3,151.1,132.4,131.0,126.4,124.3,122.1$, 122.0, 121.0, 118.6, 114.1, 14.9, 11.8. HRMS [EI]: $\mathrm{m} / \mathrm{z}$ calculated for $\mathrm{C}_{13} \mathrm{H}_{12} \mathrm{~N}_{2} \mathrm{~S}[\mathrm{M}]^{+} 228.0721$, found 228.0712. IR (ATR): $v=2924,2360,1716,1597,1508,1438,1342,1215,945,752 \mathrm{~cm}^{-1}$.

\section{2-(1H-indol-1-yl)benzo[d]thiazole (4al) ${ }^{[9]}$}

Yield: $74 \%$ (37 mg, pale white solid, 5\% ethyl acetate in petroleum ether). ${ }^{1} \mathbf{H}$ NMR $\left(300 \mathrm{MHz}, \mathrm{CDCl}_{3}\right) \delta 8.58-8.46(\mathrm{~m}, 1 \mathrm{H}), 7.91-7.80(\mathrm{~m}, 1 \mathrm{H}), 7.71$ (dd, $J=$ 8.0, $0.7 \mathrm{~Hz}, 1 \mathrm{H}), 7.63-7.51(\mathrm{~m}, 2 \mathrm{H}), 7.44-7.30(\mathrm{~m}, 2 \mathrm{H}), 7.28-7.12(\mathrm{~m}, 2 \mathrm{H})$, $6.66(\mathrm{dd}, J=3.6,0.7 \mathrm{~Hz}, 1 \mathrm{H}) .{ }^{13} \mathrm{C}-\mathrm{NMR}\left(75 \mathrm{MHz}, \mathrm{CDCl}_{3}\right) \delta 158.9,151.3,135.5$,<smiles>c1ccc2c(c1)ccn2-c1nc2ccccc2s1</smiles>
$131.5,130.4,126.6,126.5,124.5,124.3,122.8,122.0,121.3,121.1,114.3,108.2$.

\section{2-(2-methyl-1H-indol-1-yl)benzo[d]thiazole (4am)}

Yield: $72 \%$ (38mg, yellow viscous liquid, $5 \%$ ethyl acetate in petroleum ether). ${ }^{1}$ H-NMR $\left(300 \mathrm{MHz}, \mathrm{CDCl}_{3}\right) \delta 8.03-7.90(\mathrm{~m}, 2 \mathrm{H}), 7.85-7.75(\mathrm{~m}, 1 \mathrm{H}), 7.45$ (td, $J=$

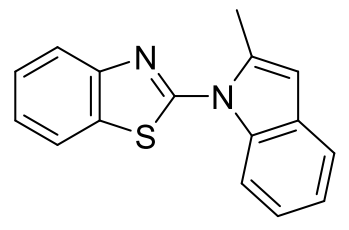


8.1, 7.7, 1.2 Hz, 2H), 7.33 (td, J= 7.7, 1.2 Hz, 1H), 7.22- $7.08(\mathrm{~m}, 2 \mathrm{H}), 6.46-6.33(\mathrm{~m}, 1 \mathrm{H}), 2.59(\mathrm{~d}, J=1.0$ $\mathrm{Hz}, 3 \mathrm{H}) .{ }^{13} \mathrm{C}-\mathrm{NMR}\left(75 \mathrm{MHz}, \mathrm{CDCl}_{3}\right) \delta 157.7,150.4,137.4,137.1,133.7,129.1,126.6,125.1,122.9,122.8$, $122.2,121.3,119.9,112.0,106.1,14.9$. HRMS [EI]: $\mathrm{m} / \mathrm{z}$ calculated for $\mathrm{C}_{16} \mathrm{H}_{12} \mathrm{~N}_{2} \mathrm{~S}[\mathrm{M}]^{+} 264.0721$, found 264.0722. IR (ATR): $V=3055,1724,1593,1508,1438,1330,1273,1199,983,752 \mathrm{~cm}^{-1}$.

\section{2-(5-bromo-1H-indol-1-yl)benzo[d]thiazole (4an)}

Yield: $82 \%$ (54 $\mathrm{mg}$, pale white solid, $20 \%$ ethyl acetate in petroleum ether). ${ }^{1} \mathrm{H}-\mathrm{NMR}\left(300 \mathrm{MHz}, \mathrm{CDCl}_{3}\right) \delta 8.52(\mathrm{~d}, J=8.8 \mathrm{~Hz}, 1 \mathrm{H}), 7.99-7.89(\mathrm{~m}, 1 \mathrm{H}), 7.84-$ $7.71(\mathrm{~m}, 2 \mathrm{H}), 7.61(\mathrm{~d}, J=3.6 \mathrm{~Hz}, 1 \mathrm{H}), 7.55-7.43(\mathrm{~m}, 2 \mathrm{H}), 7.35(\mathrm{td}, J=7.7,1.2$ $\mathrm{Hz}, 1 \mathrm{H}), 6.73-6.60(\mathrm{~m}, 1 \mathrm{H}) .{ }^{13} \mathrm{C}-\mathrm{NMR}\left(75 \mathrm{MHz}, \mathrm{CDCl}_{3}\right) \delta 158.5,151.2,134.1$, $132.0,131.4,127.4,127.3,126.7,124.5,123.8,122.2,121.2,116.0,116.0$,

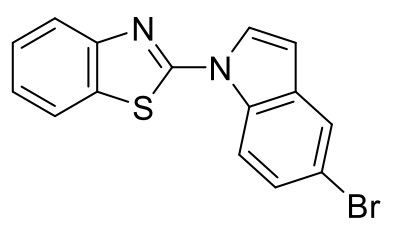
107.4. HRMS [EI]: $\mathrm{m} / \mathrm{z}$ calculated for $\mathrm{C}_{15} \mathrm{H}_{9} \mathrm{BrN}_{2} \mathrm{~S}$ [M] 327.9670 , found 327.9678. IR (ATR): $v=2924$, 2357, 1739, 1535, 1446, 1199, 1053, 914, $802 \mathrm{~cm}^{-1}$.

\section{1-(benzo[d]thiazol-2-yl)-1H-indole-4-carbonitrile (4ao)}

Yield: $85 \%$ (46 $\mathrm{mg}$, pale white solid, 15\% ethyl acetate in petroleum ether). ${ }^{1} \mathrm{H}-\mathrm{NMR}\left(300 \mathrm{MHz}, \mathrm{CDCl}_{3}\right) \delta 8.92(\mathrm{~d}, J=8.4 \mathrm{~Hz}, 1 \mathrm{H}), 7.97(\mathrm{~d}, J=8.0 \mathrm{~Hz}, 1 \mathrm{H})$, $7.90-7.71(\mathrm{~m}, 2 \mathrm{H}), 7.68-7.58(\mathrm{~m}, 1 \mathrm{H}), 7.57-7.32(\mathrm{~m}, 3 \mathrm{H}), 6.98(\mathrm{~d}, J=3.3$ $\mathrm{Hz}, 1 \mathrm{H}) .{ }^{13} \mathrm{C}-\mathrm{NMR}\left(75 \mathrm{MHz}, \mathrm{CDCl}_{3}\right) \delta$ 158.2, 151.0, 135.2, 131.8, 131.5, 128.8,

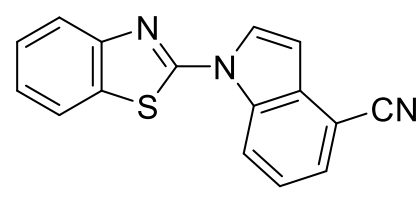
127.6, 127.0, 125.0, 124.3, 122.4, 121.3, 119.4, 117.9, 106.4, 104.0. HRMS [EI]: m/z calculated for $\mathrm{C}_{16} \mathrm{H}_{9} \mathrm{~N}_{3} \mathrm{~S}[\mathrm{M}]^{+}$275.0517, found 275.0511. IR (ATR): $v=3097,2360,2229,1735,1597,1523,1423,1330$, $1215,910 \mathrm{~cm}^{-1}$.

\section{2-(5-nitro-1H-indol-1-yl)benzo[d]thiazole (4ap)}

Yield: $90 \%$ (53 mg, yellow solid, automated column chromatography with a gradient of $0 \%$ to $20 \%$ ethyl acetate in petroleum ether). ${ }^{1} \mathbf{H}-\mathbf{N M R}$ (300 $\left.\mathrm{MHz}, \mathrm{CDCl}_{3}\right) \delta 8.75(\mathrm{~d}, J=9.2 \mathrm{~Hz}, 1 \mathrm{H}), 8.57(\mathrm{~d}, J=2.2 \mathrm{~Hz}, 1 \mathrm{H}), 8.29$ (dd, $J=$ 9.2, $2.3 \mathrm{~Hz}, 1 \mathrm{H}), 7.99(\mathrm{~d}, J=8.0 \mathrm{~Hz}, 1 \mathrm{H}), 7.85(\mathrm{~d}, J=7.7 \mathrm{~Hz}, 1 \mathrm{H}), 7.76(\mathrm{~d}, J=$ $3.6 \mathrm{~Hz}, 1 \mathrm{H}), 7.60-7.48(\mathrm{~m}, 1 \mathrm{H}), 7.47-7.34(\mathrm{~m}, 1 \mathrm{H}), 6.90(\mathrm{~d}, J=3.5 \mathrm{~Hz}, 1 \mathrm{H})$.

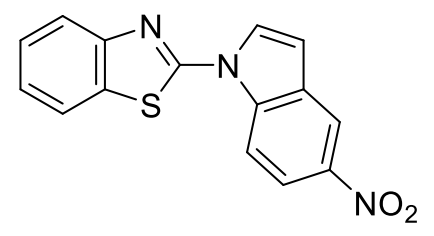

${ }^{13} \mathrm{C}-\mathrm{NMR}\left(75 \mathrm{MHz}, \mathrm{CDCl}_{3}\right) \delta 158.0,151.0,143.7,138.1,131.5,129.9,129.3,127.0,125.1,122.5,121.3$, 119.8, 117.7, 114.8, 109.1. HRMS [EI]: $\mathrm{m} / \mathrm{z}$ calculated for $\mathrm{C}_{15} \mathrm{H}_{9} \mathrm{~N}_{3} \mathrm{O}_{2} \mathrm{~S}$ [M] ${ }^{+} 295.0415$, found 295.0408. IR (ATR): $v=2931,2326,1739,1597,1508,1438,1324,1199,918,821 \mathrm{~cm}^{-1}$.

\section{2-(1H-benzo[d]imidazol-1-yl)benzo[d]thiazole $(4 a q)^{[10]}$}

Yield: $70 \%$ (35mg, pale white solid, $60 \%$ ethyl acetate in petroleum ether). ${ }^{1} \mathrm{H}$ NMR $\left(300 \mathrm{MHz}, \mathrm{CDCl}_{3}\right) \delta 8.49(\mathrm{~s}, 1 \mathrm{H}), 8.20(\mathrm{~d}, J=7.8 \mathrm{~Hz}, 1 \mathrm{H}), 7.89(\mathrm{~d}, J=7.7 \mathrm{~Hz}$, $1 \mathrm{H}), 7.83-7.69(\mathrm{~m}, 2 \mathrm{H}), 7.46-7.24(\mathrm{~m}, 4 \mathrm{H}) .{ }^{13} \mathrm{C}-\mathrm{NMR}\left(75 \mathrm{MHz}, \mathrm{CDCl}_{3}\right) \delta 155.6$, $150.4,144.3,141.3,131.9,131.7,127.1,125.3,125.3,124.5,122.7,121.4,121.0$,<smiles>c1ccc2sc(-n3cnc4ccccc43)nc2c1</smiles>
113.3. 


\section{2-(1H-pyrazol-1-yl)benzo[d]thiazole (4ar) ${ }^{[8]}$}

Yield: $54 \%$ (22 mg, colourless needles, $5 \%$ ethyl acetate in petroleum ether). ${ }^{1} \mathbf{H}$ NMR $\left(300 \mathrm{MHz}, \mathrm{CDCl}_{3}\right) \delta 8.56-8.46(\mathrm{~m}, 1 \mathrm{H}), 7.96-7.82(\mathrm{~m}, 2 \mathrm{H}), 7.79(\mathrm{~d}, J=1.3 \mathrm{~Hz}$, $1 \mathrm{H}), 7.49(\mathrm{td}, J=8.2,7.8,1.3 \mathrm{~Hz}, 1 \mathrm{H}), 7.42-7.32(\mathrm{~m}, 1 \mathrm{H}), 6.55(\mathrm{dd}, J=2.6,1.7 \mathrm{~Hz}$,

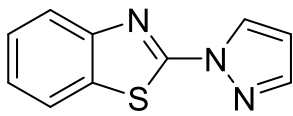
1H). ${ }^{13} \mathrm{C}-\mathrm{NMR}\left(75 \mathrm{MHz}, \mathrm{CDCl}_{3}\right) \delta 160.4,150.9,143.4,133.1,127.9,126.6,124.9,122.4,121.6,109.4$.

\section{2-(1H-imidazol-1-yl)benzo[d]thiazole (4as) ${ }^{[9]}$}

Yield: $76 \%$ (31 mg, pale white solid, $20 \%$ ethyl acetate in petroleum ether). ${ }^{1} \mathbf{H}$ NMR $\left(300 \mathrm{MHz}, \mathrm{CDCl}_{3}\right) \delta 8.30(\mathrm{~s}, 1 \mathrm{H}), 7.99-7.89(\mathrm{~m}, 1 \mathrm{H}), 7.87-7.77(\mathrm{~m}, 1 \mathrm{H}), 7.62$ $(\mathrm{t}, J=1.4 \mathrm{~Hz}, 1 \mathrm{H}), 7.51(\mathrm{td}, J=8.2,7.8,1.3 \mathrm{~Hz}, 1 \mathrm{H}), 7.40(\mathrm{td}, J=7.7,1.2 \mathrm{~Hz}, 1 \mathrm{H})$,

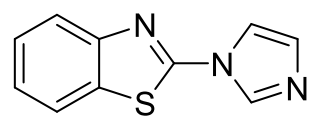
7.26 - $7.18(\mathrm{~m}, 1 \mathrm{H}) .{ }^{13} \mathrm{C}-\mathrm{NMR}\left(75 \mathrm{MHz}, \mathrm{CDCl}_{3}\right) \delta 155.9,150.5,135.9,132.1,131.3,127.1,125.6,122.8$, 121.6, 117.8 .

\section{2-(2-ethyl-4-methyl-1H-imidazol-1-yl)benzo[d]thiazole (4at)}

Yield: $24 \%$ (12 mg, pale white solid, $20 \%$ ethyl acetate in petroleum ether). ${ }^{1} \mathbf{H}$ NMR $\left(300 \mathrm{MHz}, \mathrm{CDCl}_{3}\right) \delta 8.01-7.91(\mathrm{~m}, 1 \mathrm{H}), 7.89-7.79(\mathrm{~m}, 1 \mathrm{H}), 7.52(\mathrm{td}, J=8.2$, $7.8,1.3 \mathrm{~Hz}, 1 \mathrm{H}$ ), 7.40 (td, $J=7.7,1.2 \mathrm{~Hz}, 1 \mathrm{H}$ ), 7.13 (d, $J=1.1 \mathrm{~Hz}, 1 \mathrm{H}), 3.19$ (q, $J=$ $7.5 \mathrm{~Hz}, 2 \mathrm{H}), 2.27$ (d, $J=1.1 \mathrm{~Hz}, 3 \mathrm{H}), 1.41$ (t, $J=7.5 \mathrm{~Hz}, 3 \mathrm{H}) .{ }^{13} \mathrm{C}-\mathrm{NMR}(75 \mathrm{MHz}$,

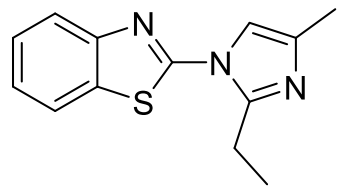
$\left.\mathrm{CDCl}_{3}\right) \delta 156.5,150.8,150.4,138.1,132.9,126.8,125.3,122.9,121.3,115.7,22.7,13.5,12.0$. HRMS [EI]: $\mathrm{m} / \mathrm{z}$ calculated for $\mathrm{C}_{13} \mathrm{H}_{13} \mathrm{~N}_{3} \mathrm{~S}[\mathrm{M}]^{+}$243.0830, found 243.0827. IR (ATR): $v=2966,2333,1739,1539$, $1438,1373,1284,1145,1064,948,713 \mathrm{~cm}^{-1}$.

\section{2-(2-phenyl-1H-imidazol-1-yl)benzo[d]thiazole (4au)}

Yield: $14 \%$ ( $8 \mathrm{mg}$, yellow viscous liquid, $20 \%$ ethyl acetate in petroleum ether). ${ }^{1} \mathbf{H}$ NMR $\left(300 \mathrm{MHz}, \mathrm{CDCl}_{3}\right) \delta 8.02-7.94(\mathrm{~m}, 1 \mathrm{H}), 7.76(\mathrm{~d}, J=1.5 \mathrm{~Hz}, 1 \mathrm{H}), 7.75-7.70$ $(\mathrm{m}, 1 \mathrm{H}), 7.62-7.56(\mathrm{~m}, 2 \mathrm{H}), 7.55-7.37(\mathrm{~m}, 5 \mathrm{H}), 7.29(\mathrm{~d}, J=1.5 \mathrm{~Hz}, 1 \mathrm{H}) .{ }^{13} \mathrm{C}-\mathrm{NMR}$ $\left(75 \mathrm{MHz}, \mathrm{CDCl}_{3}\right) \delta 156.9,149.6,147.7,134.2,130.0,129.7,129.4,128.6,126.8$, 125.8, 123.2, 121.5, 121.4. HRMS [EI]: $\mathrm{m} / \mathrm{z}$ calculated for $\mathrm{C}_{16} \mathrm{H}_{11} \mathrm{~N}_{3} \mathrm{~S}[\mathrm{M}]^{+} 277.0674$,

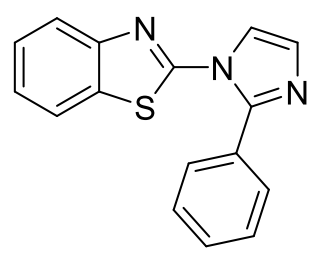
found 277.0664. IR (ATR): $V=2924,2341,1739,1508,1465,1438,1269,1246,1072,937,756 \mathrm{~cm}^{-1}$.

\section{2-(4-methoxyphenoxy)-6-methylbenzo[d]thiazole (4av) ${ }^{[4]}$}

Yield: $39 \% \quad(21 \mathrm{mg}$, brown glassy solid, automated column chromatography with a gradient of $0 \%$ to $20 \%$ ethyl acetate in petroleum ether). ${ }^{1} \mathbf{H}$ NMR $\left(300 \mathrm{MHz}, \mathrm{CDCl}_{3}\right) \delta 7.62(\mathrm{~d}, J=8.3 \mathrm{~Hz}, 1 \mathrm{H})$, $7.47-7.43(\mathrm{~m}, 1 \mathrm{H}), 7.35-7.24(\mathrm{~m}, 2 \mathrm{H}), 7.20$ (ddd, $J=8.3,1.8,0.7 \mathrm{~Hz}$, $1 \mathrm{H}), 7.03-6.91(\mathrm{~m}, 2 \mathrm{H}), 3.85(\mathrm{~s}, 3 \mathrm{H}), 2.44(\mathrm{~s}, 3 \mathrm{H}) .{ }^{13} \mathrm{C}$ NMR $(75 \mathrm{MHz}$,

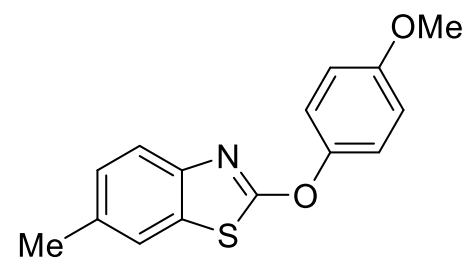
$\left.\mathrm{CDCl}_{3}\right) \delta 172.1,157.6,148.4,147.0,133.8,132.2,127.5,121.8,121.2,121.2,114.9,55.7,21.4$. 


\section{5-methoxy-2-(4-methoxyphenoxy)benzo[d]thiazole (4aw)}

Yield: 99\% (0.1 mmol scale, $28 \mathrm{mg}$, pale white solid, automated column chromatography with a gradient of $0 \%$ to $20 \%$ ethyl acetate in petroleum ether). ${ }^{1} \mathbf{H}$ NMR (600 MHz, DMSO- $\left.d_{6}\right) \delta 7.77(\mathrm{~d}, J=8.8 \mathrm{~Hz}$, $1 \mathrm{H}), 7.40-7.35(\mathrm{~m}, 2 \mathrm{H}), 7.27(\mathrm{~d}, J=2.5 \mathrm{~Hz}, 1 \mathrm{H}), 7.07-7.03(\mathrm{~m}, 2 \mathrm{H})$,

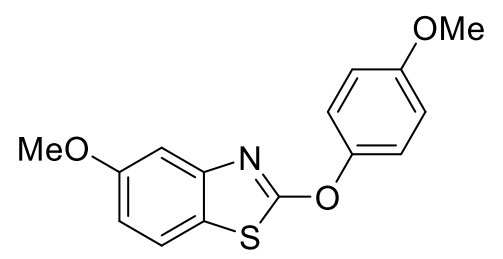
$6.94(\mathrm{dd}, J=8.8,2.6 \mathrm{~Hz}, 1 \mathrm{H}), 3.81(\mathrm{~s}, 3 \mathrm{H}), 3.79(\mathrm{~s}, 3 \mathrm{H}) .{ }^{13} \mathrm{C}$ NMR $(151$ $\mathrm{MHz}$, DMSO) $\delta 174.2,159.2,158.0,150.4,148.3,123.5,123.0,122.6,115.5,113.2,105.6,56.0,55.9$. HRMS: [EI]: $\mathrm{m} / \mathrm{z}$ calculated for $\mathrm{C}_{15} \mathrm{H}_{13} \mathrm{NO}_{3} \mathrm{~S}$ [M] ${ }^{+}$287.0616, found 287.0616. IR (ATR): 2947, 2361, 1740, $1497,1466,1435,1366,1219,1161,1026,826 \mathrm{~cm}^{-1}$.

\section{6-fluoro-2-(4-methoxyphenoxy)benzo[d]thiazole (4ax)}

Yield: $45 \%$ ( $0.1 \mathrm{mmol}$ scale, $12 \mathrm{mg}$, pale white solid, automated column chromatography with a gradient of $0 \%$ to $20 \%$ ethyl acetate in petroleum ether). ${ }^{1} \mathbf{H}$ NMR (300 MHz, $\left.\mathrm{CDCl}_{3}\right) \delta 7.67$ (dd, $\left.J=8.9,4.8 \mathrm{~Hz}, 1 \mathrm{H}\right), 7.36$ (dd, $J$ $=8.1,2.6 \mathrm{~Hz}, 1 \mathrm{H}), 7.31-7.23(\mathrm{~m}, 2 \mathrm{H}), 7.12(\mathrm{td}, J=9.0,2.7 \mathrm{~Hz}, 1 \mathrm{H}), 7.02-$ $6.91(\mathrm{~m}, 2 \mathrm{H}), 3.85(\mathrm{~s}, 3 \mathrm{H}) .{ }^{13} \mathrm{C} \mathrm{NMR}\left(75 \mathrm{MHz}, \mathrm{CDCl}_{3}\right) \delta 172.2,172.2,161.1$, $157.8,148.3,145.6,145.6,133.0,132.9,122.5,122.4,121.8,114.9,114.5$,

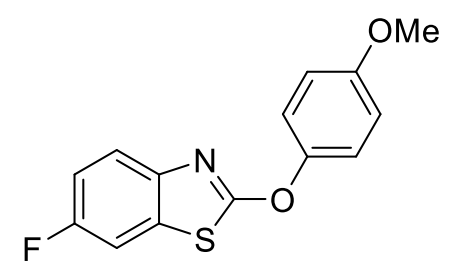
114.2, 108.0, 107.7, 55.7. HRMS: [EI]: $\mathrm{m} / \mathrm{z}$ calculated for $\mathrm{C}_{14} \mathrm{H}_{10} \mathrm{FNO} \mathrm{FS}_{2} \mathrm{M}^{+}$275.0416, found 275.0408. IR (ATR): 2936, 2361, 1740, 1601, 1535, 1504, 1450, 1227, 1030, $818 \mathrm{~cm}^{-1}$.

\section{7-bromo-2-(4-methoxyphenoxy)benzo[d]thiazole (4ay)}

Yield: $99 \%$ ( $0.1 \mathrm{mmol}$ scale, $33 \mathrm{mg}$, colourless solid, automated column chromatography with a gradient of $0 \%$ to $20 \%$ ethyl acetate in petroleum ether). ${ }^{1} \mathrm{H}$ NMR (300 MHz, $\left.\mathrm{CDCl}_{3}\right) \delta 7.68$ (dd, $J=8.0,1.0 \mathrm{~Hz}, 1 \mathrm{H}$ ), 7.39 (dd, $J=$ 7.9, $1.1 \mathrm{~Hz}, 1 \mathrm{H}), 7.33-7.26(\mathrm{~m}, 3 \mathrm{H}), 7.07-6.90(\mathrm{~m}, 2 \mathrm{H}), 3.85(\mathrm{~s}, 3 \mathrm{H}) .{ }^{13} \mathrm{C}$ NMR $\left(75 \mathrm{MHz}, \mathrm{CDCl}_{3}\right) \delta 172.6,158.0,149.3,148.2,135.0,127.4,126.6,121.9$, 120.3, 115.0, 113.5, 55.7. HRMS: [EI]: $\mathrm{m} / \mathrm{z}$ calculated for $\mathrm{C}_{14} \mathrm{H}_{10} \mathrm{BrNO}_{2} \mathrm{~S}[\mathrm{M}]^{+}$

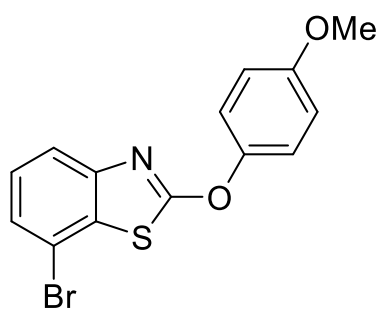
334.9616, found 334.9621. IR (ATR): 2920, 2360, 1740, 1501, 1396, 1234, 1173, 1092, 937, $833 \mathrm{~cm}^{-1}$.

\section{5-bromo-2-(4-methoxyphenoxy)benzo[d]thiazole (4az)}

Yield: $93 \%$ (0.1 mmol scale, $31 \mathrm{mg}$, pale white solid, automated column chromatography with a gradient of $0 \%$ to $20 \%$ ethyl acetate in petroleum ether). ${ }^{1} \mathbf{H}$ NMR (600 MHz, DMSO- $\left.d_{6}\right) \delta 7.92-7.87(\mathrm{~m}, 2 \mathrm{H}), 7.51-7.47$ $(\mathrm{m}, 1 \mathrm{H}), 7.42-7.37(\mathrm{~m}, 2 \mathrm{H}), 7.08-7.05(\mathrm{~m}, 2 \mathrm{H}), 3.81(\mathrm{~s}, 3 \mathrm{H}) .{ }^{13} \mathrm{C} N M R$ (151 MHz, DMSO) $\delta 174.8,158.1,150.5,148.2,131.4,127.2,124.5$,

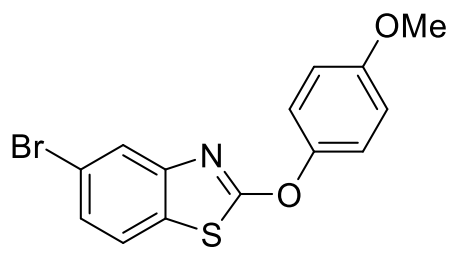
124.0, 122.6, 119.6, 115.6, 56.0. HRMS: [EI]: $\mathrm{m} / \mathrm{z}$ calculated for $\mathrm{C}_{14} \mathrm{H}_{10} \mathrm{BrNO}_{2} \mathrm{~S}[\mathrm{M}]^{+} 334.9616$, found 334.9607. IR (ATR): 2962, 2920, 2361, 1740, 1501, 1412, 1215, 1142, 1103, 1069, 833, $799 \mathrm{~cm}^{-1}$. 


\section{6-chloro-2-(4-methoxyphenoxy)benzo[d]thiazole (4ba)}

Yield: 70\% (0.1 mmol scale, $21 \mathrm{mg}$, colourless solid, automated column chromatography with a gradient of $0 \%$ to $20 \%$ ethyl acetate in petroleum ether). ${ }^{1} \mathbf{H}$ NMR $\left(300 \mathrm{MHz}, \mathrm{CDCl}_{3}\right) \delta 7.67-7.60(\mathrm{~m}, 2 \mathrm{H}), 7.38-7.31(\mathrm{~m}$, $1 \mathrm{H}), 7.31-7.23(\mathrm{~m}, 2 \mathrm{H}), 7.03-6.92(\mathrm{~m}, 2 \mathrm{H}), 3.85(\mathrm{~s}, 3 \mathrm{H}) .{ }^{13} \mathrm{C}$ NMR $(75$

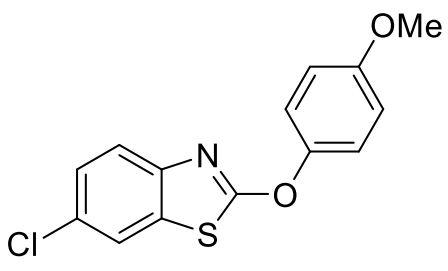
$\left.\mathrm{MHz}, \mathrm{CDCl}_{3}\right) \delta 173.1,157.8,148.2,147.8,133.3,129.4,126.9,122.4,121.8,120.9,114.9$, 55.7. HRMS: [EI]: $\mathrm{m} / \mathrm{z}$ calculated for $\mathrm{C}_{14} \mathrm{H}_{10} \mathrm{CINO}_{2} \mathrm{~S}$ [M] ${ }^{+}$291.0121, found 291.0111. IR (ATR): 2924, 2361, 1740, 1598, $1528,1501,1443,1227,1034,787 \mathrm{~cm}^{-1}$.

\section{2-(4-methoxyphenoxy)benzo[d]thiazole-5-carbonitrile (4bb)}

Yield: 93\% (0.1 mmol scale, $26 \mathrm{mg}$, brown solid, automated column chromatography with a gradient of $0 \%$ to $20 \%$ ethyl acetate in petroleum ether). ${ }^{1} \mathbf{H}$ NMR (400 MHz, $\left.\mathrm{CDCl}_{3}\right) \delta 8.01(\mathrm{~d}, J=1.5 \mathrm{~Hz}, 1 \mathrm{H})$, $7.78(\mathrm{~d}, J=8.2 \mathrm{~Hz}, 1 \mathrm{H}), 7.53(\mathrm{dd}, J=8.2,1.6 \mathrm{~Hz}, 1 \mathrm{H}), 7.36-7.24(\mathrm{~m}, 2 \mathrm{H})$, $7.06-6.92(\mathrm{~m}, 2 \mathrm{H}), 3.87(\mathrm{~s}, 3 \mathrm{H}) .{ }^{13} \mathrm{C}$ NMR (101 MHz, $\left.\mathrm{CDCl}_{3}\right) \delta 174.4$,<smiles>COc1ccc(Oc2nc3cc(C#N)ccc3s2)cc1</smiles>
$158.1,149.3,148.0,137.4,126.7,125.3,122.3,121.8,118.8,115.0,110.0,55.7$. HRMS: [EI]: $\mathrm{m} / \mathrm{z}$ calculated for $\mathrm{C}_{15} \mathrm{H}_{10} \mathrm{~N}_{2} \mathrm{O}_{2} \mathrm{~S}[\mathrm{M}]^{+}$282.0463, found 282.0462. IR (ATR): 2951, 2361, 1740, 1605, 1493, $1450,1369,1219,1026,822 \mathrm{~cm}^{-1}$.

\section{2-(4-methoxyphenoxy)benzo[d]thiazole-6-carbonitrile (4bc)}

Yield: 91\% (52 mg, colourless solid, automated column chromatography with a gradient of $0 \%$ to $20 \%$ ethyl acetate in petroleum ether). ${ }^{1} \mathbf{H}$ NMR $\left(300 \mathrm{MHz}, \mathrm{CDCl}_{3}\right) \delta 7.93-7.87(\mathrm{~m}, 1 \mathrm{H}), 7.74-7.67(\mathrm{~m}, 1 \mathrm{H}), 7.57(\mathrm{dd}, \mathrm{J}=$ 8.4, $1.7 \mathrm{~Hz}, 1 \mathrm{H}), 7.23-7.17(\mathrm{~m}, 2 \mathrm{H}), 6.94-6.87(\mathrm{~m}, 2 \mathrm{H}), 3.78(\mathrm{~s}, 3 \mathrm{H}) .{ }^{13} \mathrm{C}$ NMR $\left(75 \mathrm{MHz} \mathrm{CDCl}_{3}\right) \delta 176.1,158.1,152.5,148.0,132.8,129.9,125.7$,

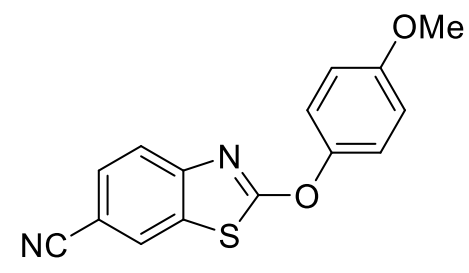
122.2, 121.8, 118.8, 115.1, 107.2, 55.7. HRMS: [EI]: $\mathrm{m} / \mathrm{z}$ calculated for $\mathrm{C}_{15} \mathrm{H}_{10} \mathrm{~N}_{2} \mathrm{O}_{2} \mathrm{~S}$ [M] ${ }^{+} 282.0463$, found 282.0460. IR (ATR): 3314, 2361, 1740, 1605, 1493, 1435, 1366, 1219, 1177, 1030, $822 \mathrm{~cm}^{-1}$.

\section{2-(4-methoxyphenoxy)-6-nitrobenzo[d]thiazole (4bd)}

Yield: $98 \%$ (0.1 mmol scale, $30 \mathrm{mg}$, yellow solid, automated column chromatography with a gradient of $0 \%$ to $20 \%$ ethyl acetate in petroleum ether). ${ }^{1} \mathbf{H}$ NMR (300 MHz, $\left.\mathrm{CDCl}_{3}\right) \delta 8.61(\mathrm{~d}, J=2.3 \mathrm{~Hz}, 1 \mathrm{H})$, $8.29(\mathrm{dd}, J=8.9,2.4 \mathrm{~Hz}, 1 \mathrm{H}), 7.80(\mathrm{~d}, J=9.0 \mathrm{~Hz}, 1 \mathrm{H}), 7.42-7.19(\mathrm{~m}, 2 \mathrm{H})$, $7.09-6.91(\mathrm{~m}, 2 \mathrm{H}), 3.87(\mathrm{~s}, 3 \mathrm{H}) .{ }^{13} \mathrm{C}$ NMR $\left(75 \mathrm{MHz}, \mathrm{CDCl}_{3}\right) \delta 177.0$, 158.2, 154.1, 147.9, 144.0, 132.5, 122.1, 121.8, 121.6, 117.8, 115.1,

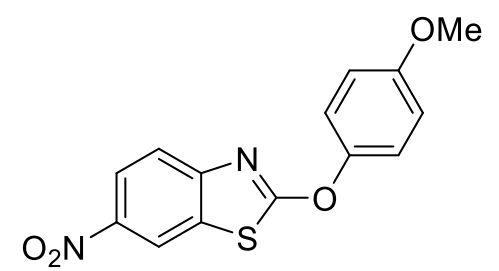
55.7. HRMS: [EI]: $\mathrm{m} / \mathrm{z}$ calculated for $\mathrm{C}_{14} \mathrm{H}_{10} \mathrm{~N}_{2} \mathrm{O}_{4} \mathrm{~S}$ [M] ${ }^{+}$302.0361, found 302.0356. IR (ATR): 2924, 2361, $1740,1493,1443,1335,1231,1180,1103,1015,826 \mathrm{~cm}^{-1}$. 


\section{6-methyl-2-(5-nitro-1H-indol-1-yl)benzo[d]thiazole (4be)}

Yield: $83 \%$ (0.1 mmol scale, $26 \mathrm{mg}$, yellow solid, automated column chromatography with a gradient of $0 \%$ to $20 \%$ ethyl acetate in petroleum ether). ${ }^{1} \mathbf{H}$ NMR (600 MHz, DMSO- $\left.d_{6}\right) \delta 8.72(d, J=9.1 \mathrm{~Hz}$, $1 \mathrm{H}), 8.67(\mathrm{~d}, J=2.1 \mathrm{~Hz}, 1 \mathrm{H}), 8.28(\mathrm{dd}, J=9.2,2.3 \mathrm{~Hz}, 1 \mathrm{H}), 8.15(\mathrm{~d}, J=$ $3.5 \mathrm{~Hz}, 1 \mathrm{H}), 7.93-7.86(\mathrm{~m}, 2 \mathrm{H}), 7.39$ (dd, $J=8.3,1.7 \mathrm{~Hz}, 1 \mathrm{H}), 7.14$ (d, $J$

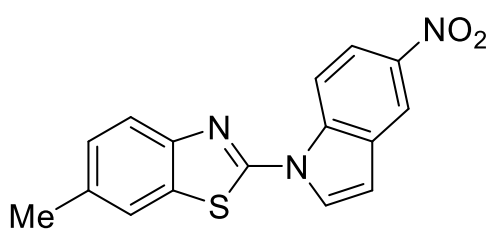
$=3.6 \mathrm{~Hz}, 1 \mathrm{H}), 2.47(\mathrm{~s}, 3 \mathrm{H}) .{ }^{13} \mathrm{C}$ NMR $\left(151 \mathrm{MHz}, \mathrm{DMSO}-d_{6}\right) \delta 158.0,148.9,143.7,138.1,135.4,132.1$, 131.0, 130.4, 128.8, 122.2, 122.1, 119.8, 118.2, 115.1, 109.7, 21.5. HRMS: [EI]: $\mathrm{m} / \mathrm{z}$ calculated for $\mathrm{C}_{16} \mathrm{H}_{11} \mathrm{~N}_{3} \mathrm{O}_{2} \mathrm{~S}[\mathrm{M}]^{+}$309.0572, found 309.0564. IR (ATR): 2920, 2851, 2361, 1740, 1597, 1512, 1443, 1327, $1200,918,876,810 \mathrm{~cm}^{-1}$.

\section{5-methoxy-2-(5-nitro-1H-indol-1-yl)benzo[d]thiazole (4bf)}

Yield: $68 \%$ (0.1 mmol scale, $22 \mathrm{mg}$, pale yellow solid, automated column chromatography with a gradient of $0 \%$ to $20 \%$ ethyl acetate in petroleum ether). ${ }^{1} \mathbf{H}$ NMR $\left(600 \mathrm{MHz}\right.$, DMSO- $\left.d_{6}\right) \delta 8.78(\mathrm{~d}, J=9.1$<smiles>COc1ccc2sc(-n3ccc4cc([N+](=O)[O-])ccc43)nc2c1</smiles>
$\mathrm{Hz}, 1 \mathrm{H}), 8.70(\mathrm{~d}, J=2.3 \mathrm{~Hz}, 1 \mathrm{H}), 8.30(\mathrm{dd}, J=9.2,2.4 \mathrm{~Hz}, 1 \mathrm{H}), 8.20(\mathrm{~d}, J=3.6 \mathrm{~Hz}, 1 \mathrm{H}), 8.01(\mathrm{~d}, J=8.8 \mathrm{~Hz}$, $1 \mathrm{H}), 7.57(\mathrm{~d}, J=2.5 \mathrm{~Hz}, 1 \mathrm{H}$ ), 7.15 (dd, $J=3.6,0.7 \mathrm{~Hz}, 1 \mathrm{H}$ ), 7.09 (dd, $J=8.8,2.5 \mathrm{~Hz}, 1 \mathrm{H}), 3.88(\mathrm{~s}, 3 \mathrm{H}) .{ }^{13} \mathrm{C}$ NMR (151 MHz, DMSO) $\delta 160.1,159.6,152.2,143.5,138.0,131.1,130.4,123.3,123.1,119.9,118.3$, 115.3, 114.9, 109.9, 105.8, 56.1. HRMS: [EI]: $\mathrm{m} / \mathrm{z}$ calculated for $\mathrm{C}_{16} \mathrm{H}_{11} \mathrm{~N}_{3} \mathrm{O}_{3} \mathrm{~S}$ [M] 325.0521 , found 325.0521. IR (ATR): 2924, 2357, 1740, 1605, 1508, 1435, 1315, 1281, 1192, 1069, 957, $822 \mathrm{~cm}^{-1}$.

\section{6-fluoro-2-(5-nitro-1H-indol-1-yl)benzo[d]thiazole (4bg)}

Yield: 51\% (0.1 mmol scale, $16 \mathrm{mg}$, yellow solid, automated column chromatography with a gradient of $0 \%$ to $20 \%$ ethyl acetate in petroleum ether). ${ }^{1} \mathbf{H}$ NMR (600 MHz, DMSO- $\left.d_{6}\right) \delta 8.77(\mathrm{~d}, J=9.1 \mathrm{~Hz}$, $1 \mathrm{H}), 8.71(\mathrm{~d}, J=2.3 \mathrm{~Hz}, 1 \mathrm{H}), 8.32(\mathrm{dd}, J=9.1,2.4 \mathrm{~Hz}, 1 \mathrm{H}), 8.25(\mathrm{~d}, J=3.6$ $\mathrm{Hz}, 1 \mathrm{H}), 8.12(\mathrm{dd}, J=8.7,2.7 \mathrm{~Hz}, 1 \mathrm{H}), 8.05(\mathrm{dd}, J=8.9,4.8 \mathrm{~Hz}, 1 \mathrm{H}), 7.45$<smiles>O=[N+]([O-])c1ccc2c(ccn2-c2nc3ccc(F)cc3s2)c1</smiles>
$(\mathrm{td}, J=9.1,2.7 \mathrm{~Hz}, 1 \mathrm{H}), 7.18(\mathrm{~d}, J=3.5 \mathrm{~Hz}, 1 \mathrm{H}) .{ }^{13} \mathrm{C}$ NMR $(151 \mathrm{MHz}$, DMSO-d $) \delta 160.7,159.1,159.1$, 147.7, 143.6, 138.0, 133.2, 133.1, 131.3, 130.4, 123.7, 123.7, 120.0, 118.3, 115.9, 115.7, 115.3, 110.0, 109.6, 109.4. HRMS: [EI]: $\mathrm{m} / \mathrm{z}$ calculated for $\mathrm{C}_{15} \mathrm{H}_{8} \mathrm{FN}_{3} \mathrm{O}_{2} \mathrm{~S}$ [M] ${ }^{+} 313.0321$, found 313.0319. IR (ATR): 2924, $2855,2361,1740,1605,1508,1447,1319,1192,1072,930,887,806 \mathrm{~cm}^{-1}$.

\section{7-bromo-2-(5-nitro-1H-indol-1-yl)benzo[d]thiazole (4bh)}

Yield: $98 \%$ ( $0.1 \mathrm{mmol}$ scale, $36 \mathrm{mg}$, pale yellow solid, automated column chromatography with a gradient of $0 \%$ to $20 \%$ ethyl acetate in petroleum ether). ${ }^{1} \mathbf{H}$ NMR (600 MHz, DMSO- $\left.d_{6}\right) \delta 8.79(\mathrm{~d}, J=9.1 \mathrm{~Hz}, 1 \mathrm{H}), 8.70(\mathrm{~d}, J=$ $2.3 \mathrm{~Hz}, 1 \mathrm{H}), 8.35-8.31(\mathrm{~m}, 2 \mathrm{H}), 8.04(\mathrm{dd}, J=8.1,0.9 \mathrm{~Hz}, 1 \mathrm{H}), 7.70(\mathrm{dd}, J=$ 7.9, $0.9 \mathrm{~Hz}, 1 \mathrm{H}), 7.55(\mathrm{t}, J=8.0 \mathrm{~Hz}, 1 \mathrm{H}), 7.19(\mathrm{~d}, J=3.7 \mathrm{~Hz}, 1 \mathrm{H}) .{ }^{13} \mathrm{C}$ NMR $\left(151 \mathrm{MHz}, \mathrm{DMSO}-d_{6}\right) \delta 158.4,150.9,143.8,138.0,134.5,131.4,130.7$,<smiles>O=[N+]([O-])c1ccc2c(ccn2-c2nc3cccc(Br)c3s2)c1</smiles> 
129.3, 128.2, 121.7, 120.2, 118.3, 115.6, 113.3, 110.4. HRMS: [EI]: $\mathrm{m} / \mathrm{z}$ calculated for $\mathrm{C}_{15} \mathrm{H}_{8} \mathrm{BrN}_{3} \mathrm{O}_{2} \mathrm{~S}$ [M] 372.9521, found 372.9521. IR (ATR): 2924, 2357, 1736, 1589, 1516, 1439, 1323, 1192, 930, $822 \mathrm{~cm}^{-1}$.

\section{5-bromo-2-(5-nitro-1H-indol-1-yl)benzo[d]thiazole (4bi)}

Yield: $78 \%(0.1 \mathrm{mmol}$ scale, $29 \mathrm{mg}$, pale yellow solid, automated column chromatography with a gradient of $0 \%$ to $20 \%$ ethyl acetate in petroleum ether). ${ }^{1} \mathbf{H}$ NMR (400 MHz, DMSO- $\left.d_{6}\right) \delta 8.77(\mathrm{~d}, J=9.2 \mathrm{~Hz}$, $1 \mathrm{H}), 8.69(\mathrm{~d}, J=2.3 \mathrm{~Hz}, 1 \mathrm{H}), 8.31(\mathrm{dd}, J=9.2,2.4 \mathrm{~Hz}, 1 \mathrm{H}), 8.22(\mathrm{dd}, J=$ $5.9,2.8 \mathrm{~Hz}, 2 \mathrm{H}), 8.14(\mathrm{~d}, J=8.6 \mathrm{~Hz}, 1 \mathrm{H}), 7.63(\mathrm{dd}, J=8.5,1.9 \mathrm{~Hz}, 1 \mathrm{H})$,

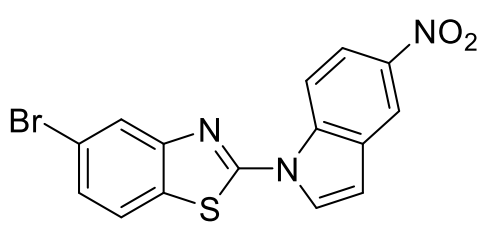
$7.17(\mathrm{~d}, J=3.6 \mathrm{~Hz}, 1 \mathrm{H}) .{ }^{13} \mathrm{C}$ NMR $\left(151 \mathrm{MHz}\right.$, DMSO- $\left.d_{6}\right) \delta 160.6,152.2,143.9,138.1,131.3,131.1,130.6$, 128.3, 124.9, 124.5, 120.3, 120.0, 118.2, 115.3, 110.2. HRMS: [EI]: $\mathrm{m} / \mathrm{z}$ calculated for $\mathrm{C}_{15} \mathrm{H}_{8} \mathrm{BrN}_{3} \mathrm{O}_{2} \mathrm{~S}$ [M] 372.9521, found 372.9522. IR (ATR): 2920, 2334, 1736, 1535, 1508, 1439, 1327, 1065, 922, $822 \mathrm{~cm}^{-1}$.

\section{6-chloro-2-(5-nitro-1H-indol-1-yl)benzo[d]thiazole (4bj)}

Yield: $99 \%$ (0.1 mmol scale, $32 \mathrm{mg}$, yellow solid, automated column chromatography with a gradient of $0 \%$ to $20 \%$ ethyl acetate in petroleum ether). ${ }^{1} \mathbf{H}$ NMR $\left(250 \mathrm{MHz}, \mathrm{DMSO}-d_{6}\right) \delta 8.83-8.64(\mathrm{~m}, 2 \mathrm{H})$, $8.40-8.25(\mathrm{~m}, 2 \mathrm{H}), 8.23-8.12(\mathrm{~m}, 1 \mathrm{H}), 8.01(\mathrm{~d}, J=8.6 \mathrm{~Hz}, 1 \mathrm{H}), 7.60(\mathrm{~d}$, $J=8.7 \mathrm{~Hz}, 1 \mathrm{H}), 7.17(\mathrm{~d}, J=3.6 \mathrm{~Hz}, 1 \mathrm{H}) .{ }^{13} \mathrm{C}$ NMR $(63 \mathrm{MHz}, \mathrm{DMSO}) \delta$

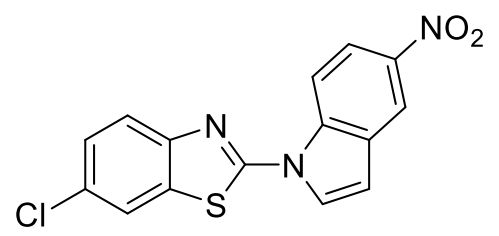
$160.7,150.6,144.8,139.0,134.5,132.0,131.5,130.7,128.9,124.5,123.3,120.9,119.1,116.2,111.0$. HRMS: [EI]: $\mathrm{m} / \mathrm{z}$ calculated for $\mathrm{C}_{15} \mathrm{H}_{8} \mathrm{ClN}_{3} \mathrm{O}_{2} \mathrm{~S}$ [M] ${ }^{+} 329.0026$, found 329.0024. IR (ATR): 2924, 2342, 1740, $1597,1512,1443,1323,1200,918,810 \mathrm{~cm}^{-1}$.

\section{$N, 1,1,1-$ tetraphenyl- $\lambda^{5}$-phosphanimine $(5)^{[11]}$}

Yield: 56\% (40 mg, pale yellow oil, 7\% ethyl acetate in petroleum ether). ${ }^{1} \mathbf{H}$ NMR (300 $\left.\mathrm{MHz}, \mathrm{CDCl}_{3}\right) \delta 7.84-7.73(\mathrm{~m}, 6 \mathrm{H}), 7.55-7.43(\mathrm{~m}, 9 \mathrm{H}), 7.08-6.98(\mathrm{~m}, 2 \mathrm{H}), 6.88-6.78$ $(\mathrm{m}, 2 \mathrm{H}), 6.72-6.62(\mathrm{~m}, 1 \mathrm{H}) .{ }^{13} \mathrm{C}$ NMR $\left(75 \mathrm{MHz}, \mathrm{CDCl}_{3}\right) \delta 151.1,151.0,132.7,132.6$,<smiles>P=Nc1ccccc1</smiles>
$132.2,132.0,131.7,131.7,130.4,128.7,128.6,128.6,128.5,128.5,123.6,123.4,117.4$.

\section{General procedure for the 2-D-benzothiazoles}

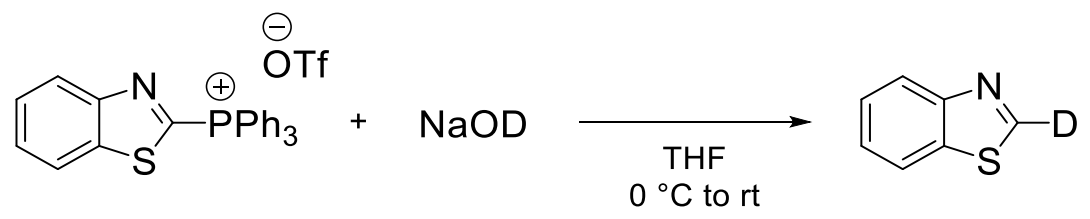

Sodium deuteroxide $(0.25 \mathrm{mmol})$ was added to the phosphonium salt $(0.1 \mathrm{mmol})$ in dry THF $(0.1 \mathrm{M})$ under inert atmosphere at $0{ }^{\circ} \mathrm{C}$. The reaction mixture was warmed up to room temperature and quenched by addition of a small amount of water when it completed. The crude product was purified by column chromatography using ethyl acetate in petroleum ether as the eluent (Known products were compared with the literature and matched the analytical data). 


\section{benzo[d]thiazole-2-d $(6 a)^{[12]}$}

Yield: $78 \%$ (11 mg, colourless oil, 5\% ethyl acetate in petroleum ether). ${ }^{1} \mathbf{H}$ NMR (300 $\left.\mathrm{MHz}, \mathrm{CDCl}_{3}\right) \delta 8.23-8.10(\mathrm{~m}, 1 \mathrm{H}), 8.06-7.92(\mathrm{~m}, 1 \mathrm{H}), 7.62-7.36(\mathrm{~m}, 2 \mathrm{H}) .{ }^{13} \mathrm{C}$ NMR $(75$ $\left.\mathrm{MHz}, \mathrm{CDCl}_{3}\right) \delta 153.2,133.6,126.2,125.5,123.6,121.9$.

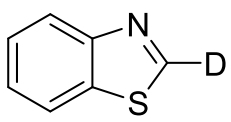

\section{6-methylbenzo[d]thiazole-2-d (6b) ${ }^{[12]}$}

Yield: $97 \%$ (15 mg, colourless oil, $5 \%$ ethyl acetate in petroleum ether). ${ }^{1} \mathbf{H}$ NMR $\left(300 \mathrm{MHz}, \mathrm{CDCl}_{3}\right) \delta 8.03(\mathrm{~d}, J=8.3 \mathrm{~Hz}, 1 \mathrm{H}), 7.81-7.66(\mathrm{~m}, 1 \mathrm{H}), 7.34$ (dd, $J=8.4$, $1.7 \mathrm{~Hz}, 1 \mathrm{H}), 2.52(\mathrm{~s}, 3 \mathrm{H}) .{ }^{13} \mathrm{C}$ NMR $\left(75 \mathrm{MHz}, \mathrm{CDCl}_{3}\right) \delta 151.4,135.7,133.8,127.8$,<smiles>[2H]c1nc2ccc(C)cc2s1</smiles>
$123.0,121.5,21.5$.

\section{benzo[d]thiazole-6-carbonitrile-2-d (6c)}

Yield: $68 \%$ (11 mg, colourless oil, $10 \%$ ethyl acetate in petroleum ether). ${ }^{1} \mathbf{H}$ NMR $\left(300 \mathrm{MHz}, \mathrm{CDCl}_{3}\right) \delta 8.34$ (dd, $J=1.6,0.7 \mathrm{~Hz}, 1 \mathrm{H}$ ), 8.23 (dd, $J=8.5,0.7 \mathrm{~Hz}, 1 \mathrm{H}$ ), 7.79 (dd, $J=8.5,1.6 \mathrm{~Hz}, 1 \mathrm{H}) .{ }^{13} \mathrm{C}$ NMR $\left(75 \mathrm{MHz}, \mathrm{CDCl}_{3}\right) \delta 155.5,134.3,129.3,127.0$,<smiles>[2H]c1nc2ccc(C#N)cc2s1</smiles>
124.6, 118.5, 109.3. HRMS: [EI]: $\mathrm{m} / \mathrm{z}$ calculated for $\mathrm{C}_{8} \mathrm{H}_{3} \mathrm{DN}_{2} \mathrm{~S}$ [M] ${ }^{+}$161.0158, found 161.0152. IR (ATR): $2920,2851,2230,1921,1462,1431,1396,1188,1138,1065,976,903,826 \mathrm{~cm}^{-1}$.

\section{References}

[1] Anders, E.; Markus, F., Chem. Ber. 1989, 122, 113-118.

[2] Q. Liu, Z. Lu, W. Ren, K. Shen, Y. Wang, Q. Xu, Chin. J. Chem. 2013, 31, 764-772.

[3] R. Huang, Y. Huang, X. Lin, M. Rong, Z. Weng, Angew. Chem. Int. Ed. 2015, 54, 5736-5739.

[4] S. Murru, P. Mondal, R. Yella, B. K. Patel, Eur. J. Org. Chem. 2009, 2009, 5406-5413.

[5] S. Bhilare, S. S. Murthy Bandaru, J. Shah, N. Chrysochos, C. Schulzke, Y. S. Sanghvi, A. R. Kapdi, J. Org. Chem. 2018, 83, 13088-13102.

[6] D. Monguchi, T. Fujiwara, H. Furukawa, A. Mori, Org. Lett. 2009, 11, 1607-1610.

[7] Y. Xu, B. Li, X. Zhang, X. Fan, J. Org. Chem. 2017, 82, 9637-9646.

[8] S. Toulot, T. Heinrich, F. R. Leroux, Adv. Synth. Catal. 2013, 355, 3263-3272.

[9] D. Ma, X. Lu, L. Shi, H. Zhang, Y. Jiang, X. Liu, Angew. Chem. Int. Ed. 2011, 50, 1118-1121.

[10] R. T. McGuire, J. F. Paffile, Y. Zhou, M. Stradiotto, ACS Catal. 2019, 9, 9292-9297.

[11] R. S. Foster, H. Adams, H. Jakobi, J. P. Harrity, J. Org. Chem. 2013, 78, 4049-4064.

[12] M. Arisawa, F. Toriyama, M. Yamaguchi, Tetrahedron Lett. 2011, 52, 2344-2347. 


\section{Copies of NMR spectra}

benzo[d]thiazol-2-yltriphenylphosphonium trifluoromethanesulfonate (2a)

${ }^{1} \mathrm{H} \mathrm{NMR}(400 \mathrm{MHz}, \mathrm{CDCl} 3)$ and ${ }^{13} \mathrm{C} \mathrm{NMR}(101 \mathrm{MHz}, \mathrm{CDCl} 3)$

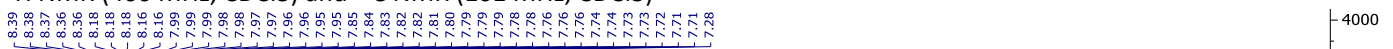
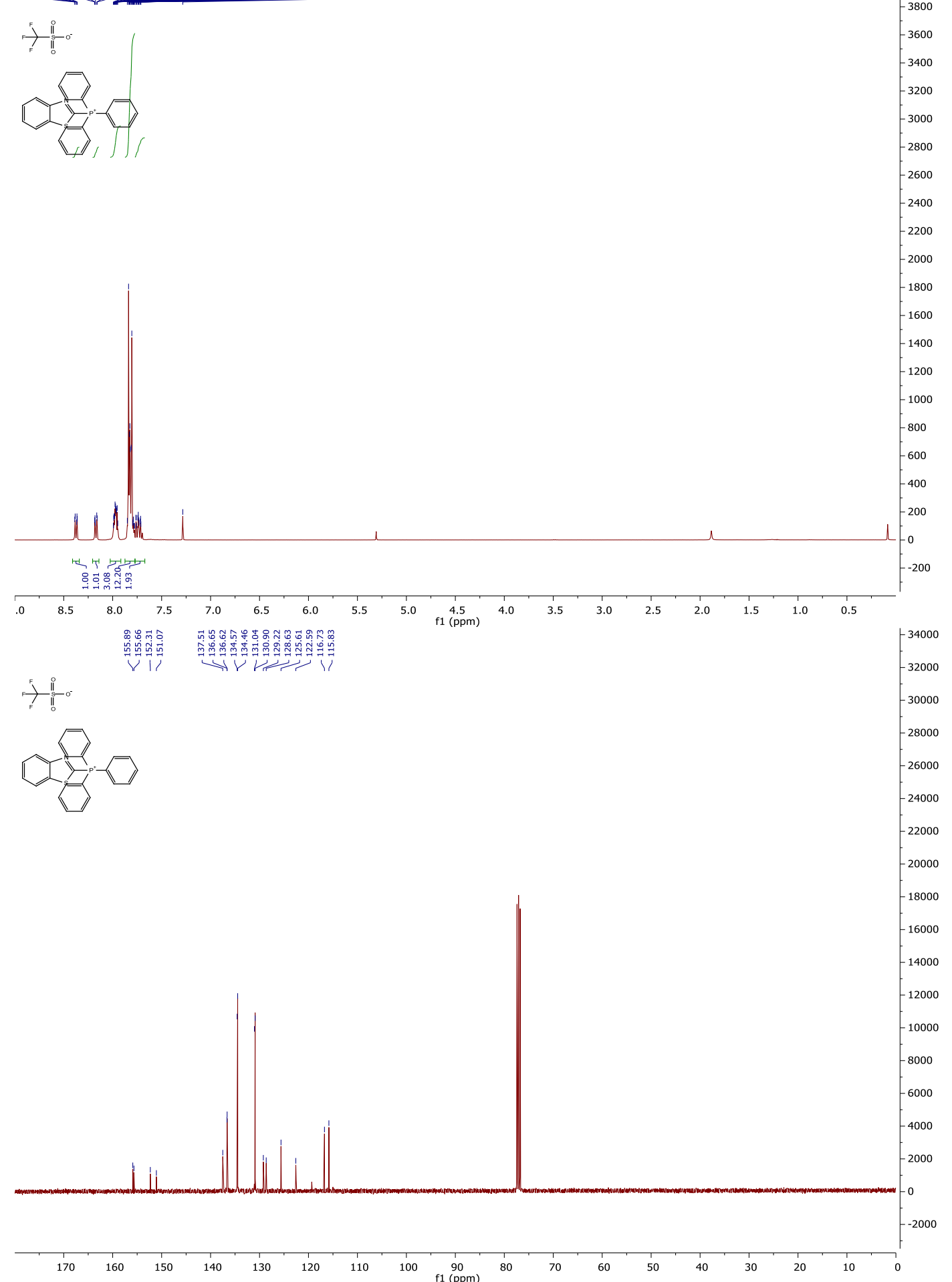
(6-methylbenzo[d]thiazol-2-yl)triphenylphosphonium trifluoromethanesulfonate (2b)

${ }^{1} \mathrm{H}$ NMR (400 MHz, CDCl3), ${ }^{13} \mathrm{C}$ NMR (101 MHz, CDCl3), ${ }^{19} \mathrm{~F} \mathrm{NMR} \mathrm{(377} \mathrm{MHz,} \mathrm{CDCl3)} \mathrm{and}{ }^{31} \mathrm{P} \mathrm{NMR}(162 \mathrm{MHz}, \mathrm{CDCl} 3)$

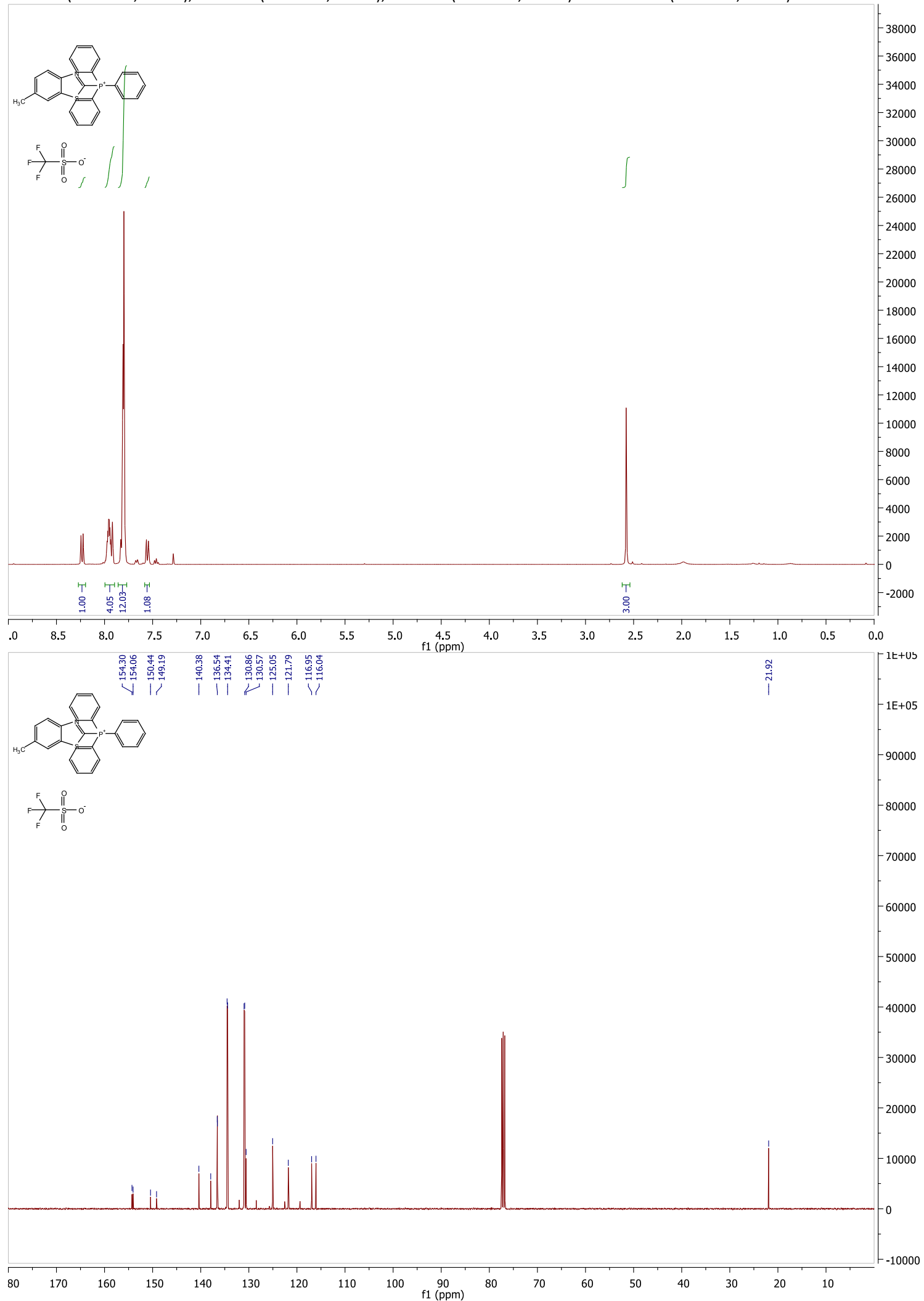




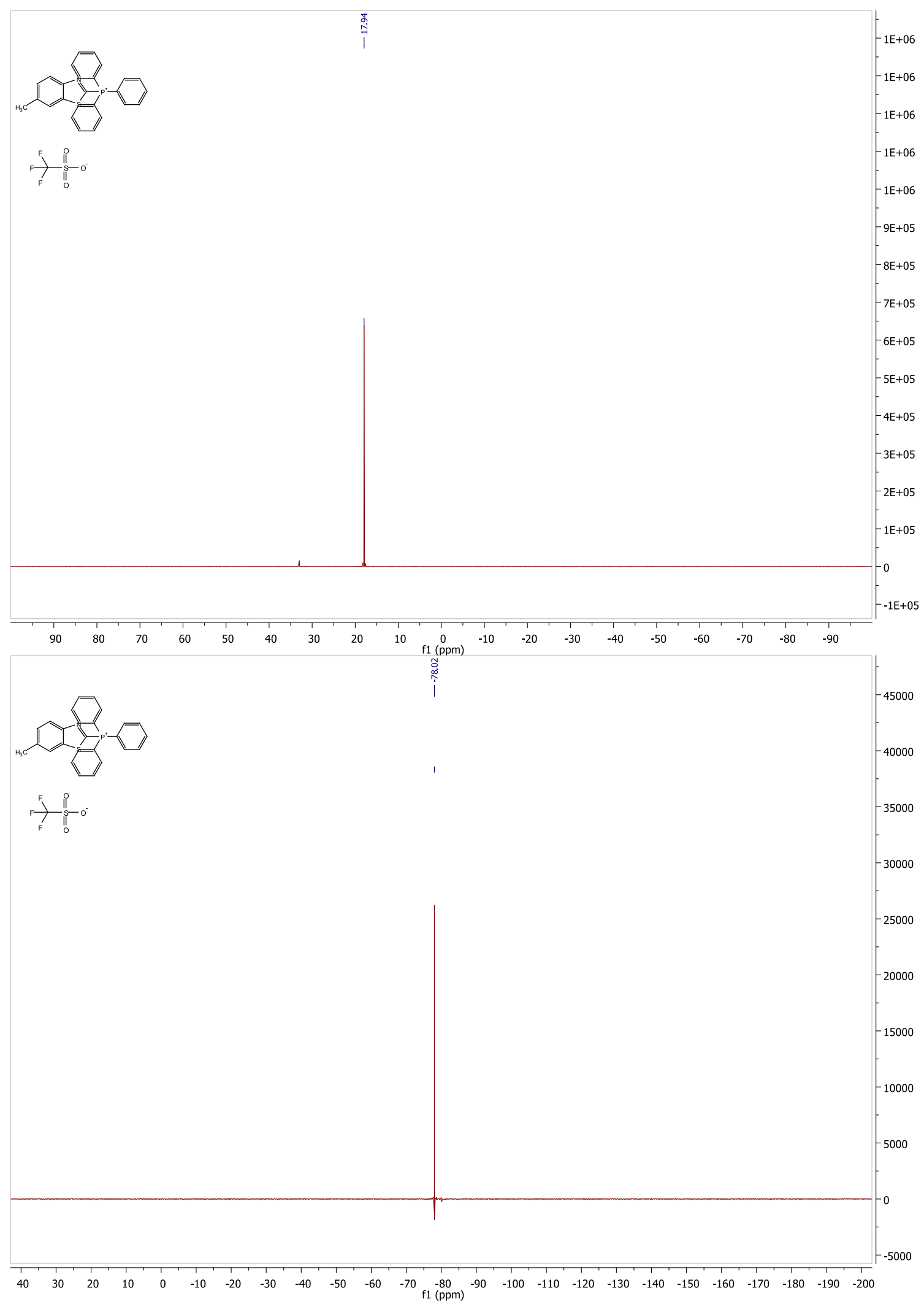


(5-methoxybenzo[d]thiazol-2-yl)triphenylphosphonium trifluoromethanesulfonate (2c)

${ }^{1} \mathrm{H}$ NMR (400 MHz, CDCl3), ${ }^{13} \mathrm{C} \mathrm{NMR}(101 \mathrm{MHz}, \mathrm{CDCl} 3),{ }^{19} \mathrm{~F} \mathrm{NMR}(377 \mathrm{MHz}, \mathrm{CDCl} 3)$ and ${ }^{31} \mathrm{P} \mathrm{NMR}(162 \mathrm{MHz}, \mathrm{CDCl} 3)$

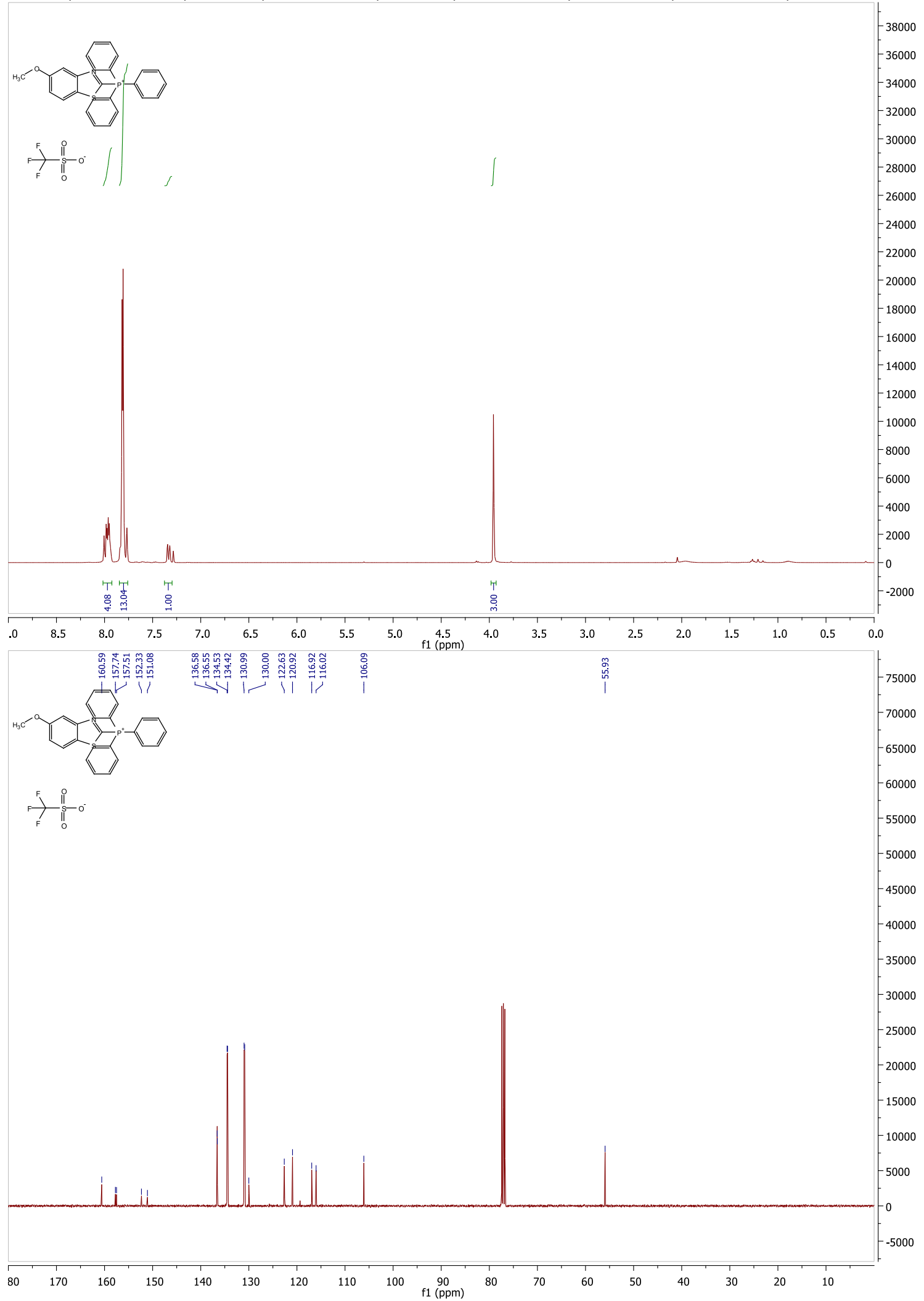




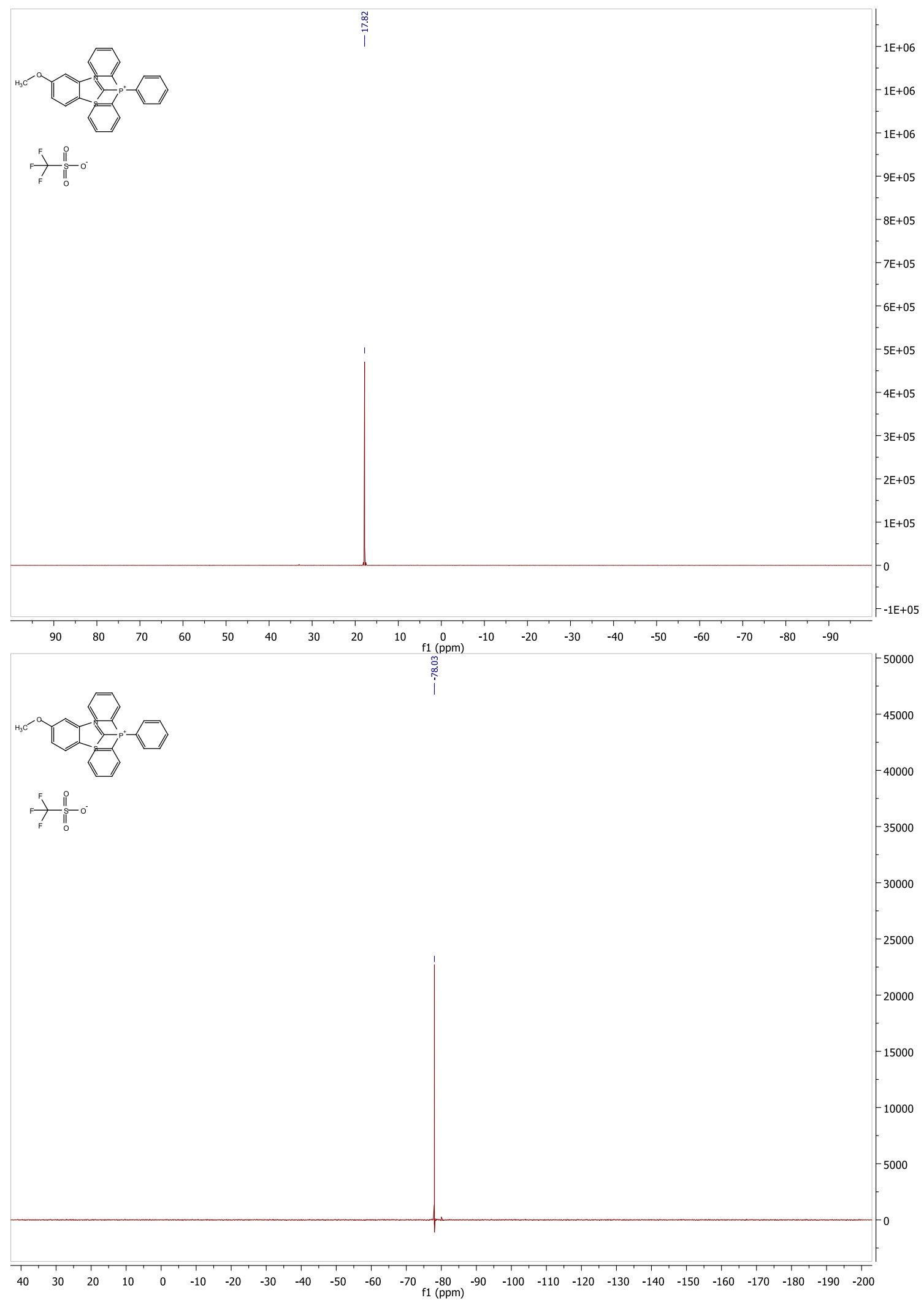


(6-fluorobenzo[d]thiazol-2-yl)triphenylphosphonium trifluoromethanesulfonate (2d)

${ }^{1} \mathrm{H}$ NMR (400 MHz, CDCl3), ${ }^{13} \mathrm{C} \mathrm{NMR} \mathrm{(101} \mathrm{MHz,} \mathrm{CDCl3),}{ }^{19} \mathrm{~F} \mathrm{NMR} \mathrm{(377} \mathrm{MHz,} \mathrm{CDCl3)} \mathrm{and}{ }^{31} \mathrm{P} \mathrm{NMR} \mathrm{(162} \mathrm{MHz,} \mathrm{CDCl3)}$

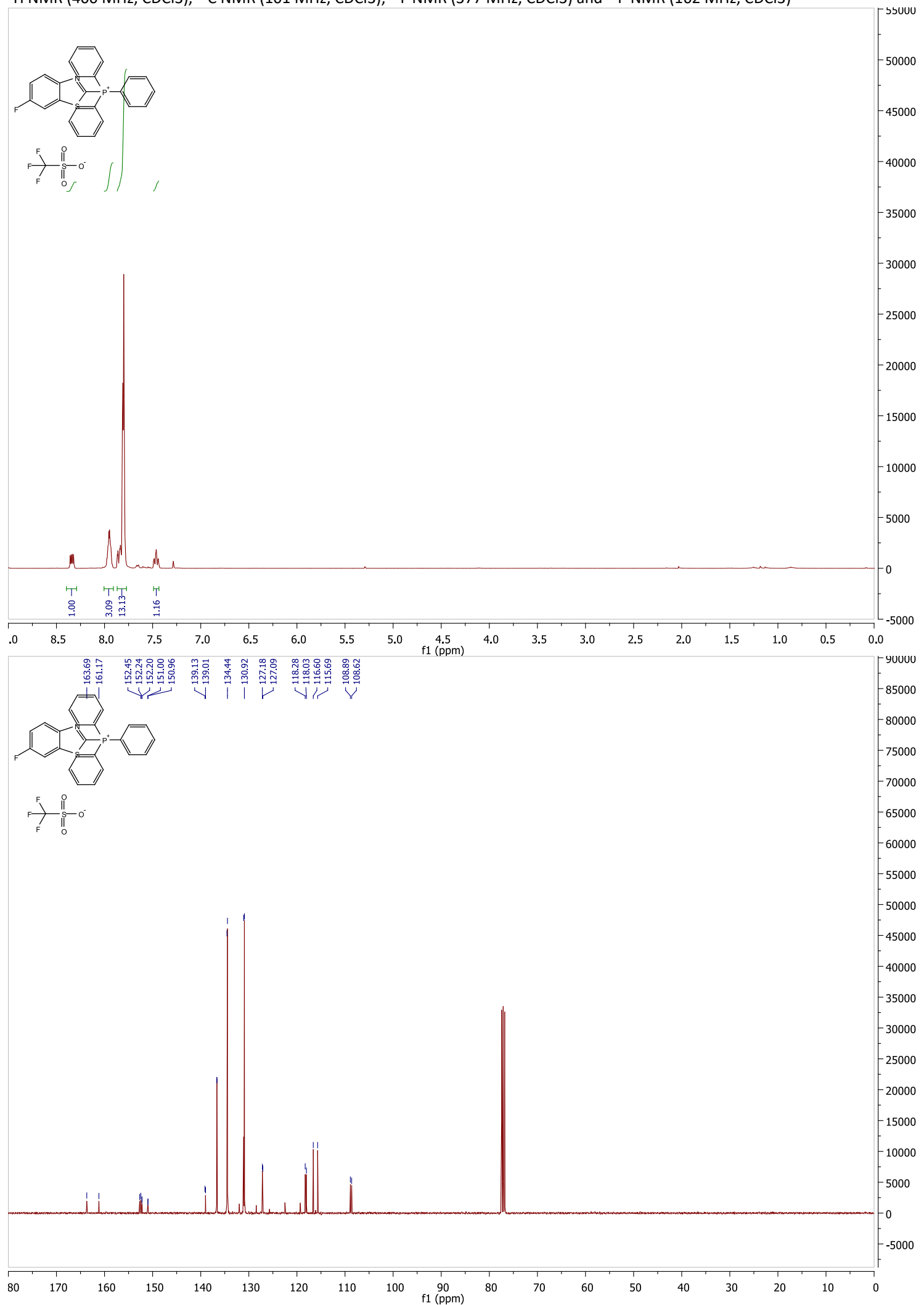




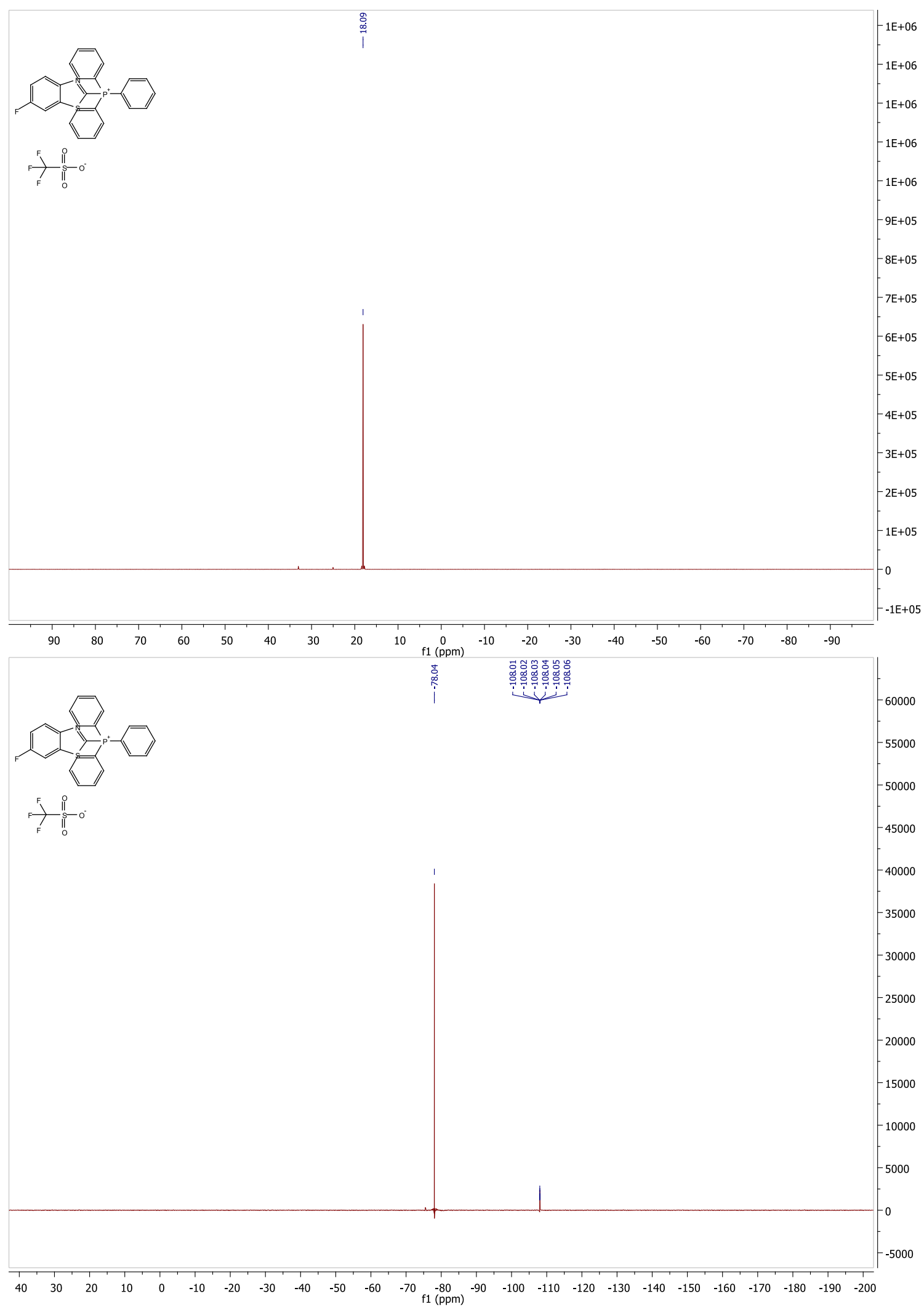


(7-bromobenzo[d]thiazol-2-yl)triphenylphosphonium trifluoromethanesulfonate (2e)

${ }^{1} \mathrm{H}$ NMR (400 MHz, CDCl3), ${ }^{13} \mathrm{C} \mathrm{NMR} \mathrm{(101} \mathrm{MHz,} \mathrm{CDCl3),}{ }^{19} \mathrm{~F} \mathrm{NMR} \mathrm{(377} \mathrm{MHz,} \mathrm{CDCl3)} \mathrm{and}{ }^{31} \mathrm{P} \mathrm{NMR} \mathrm{(162} \mathrm{MHz,} \mathrm{CDCl3)}$

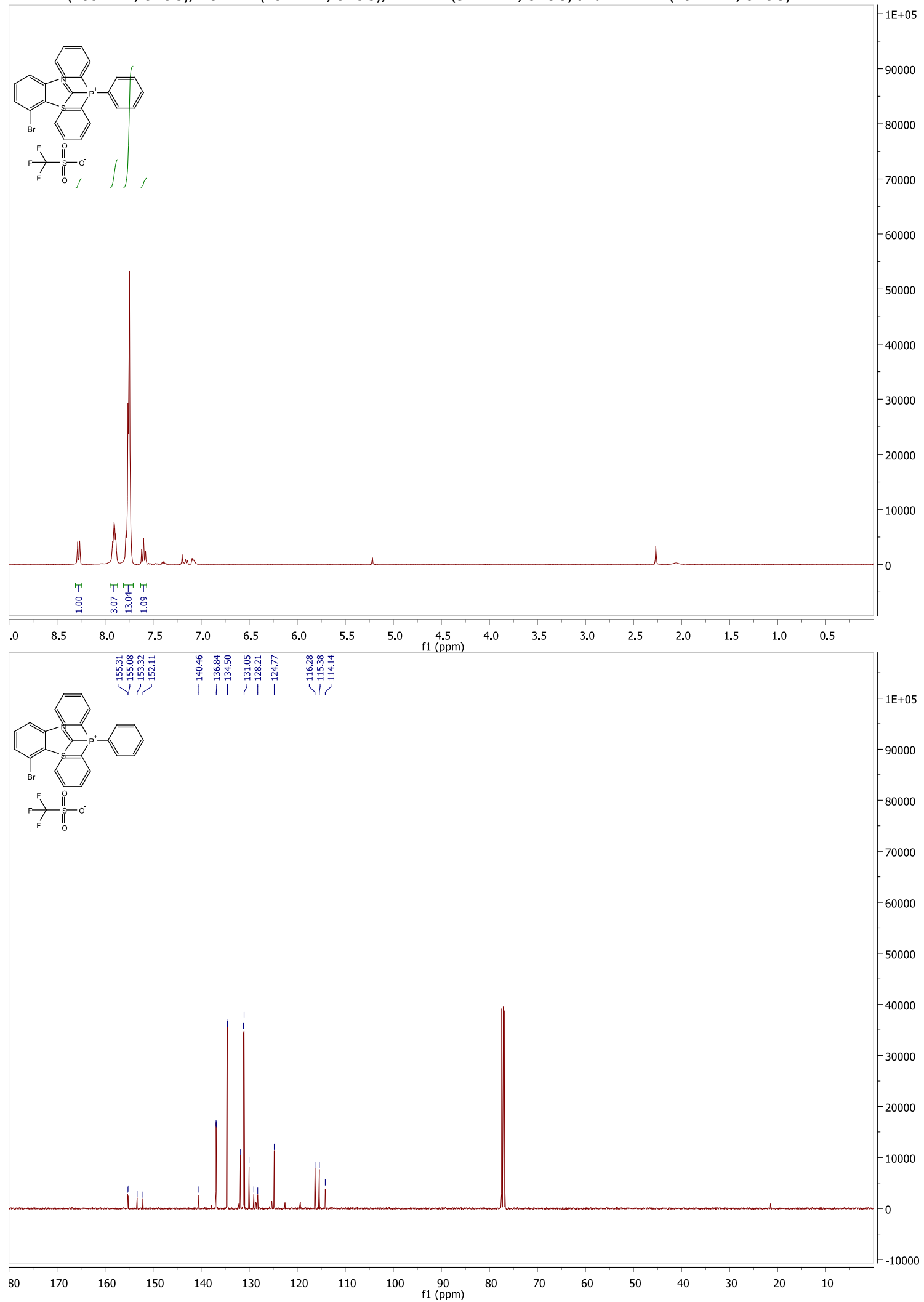




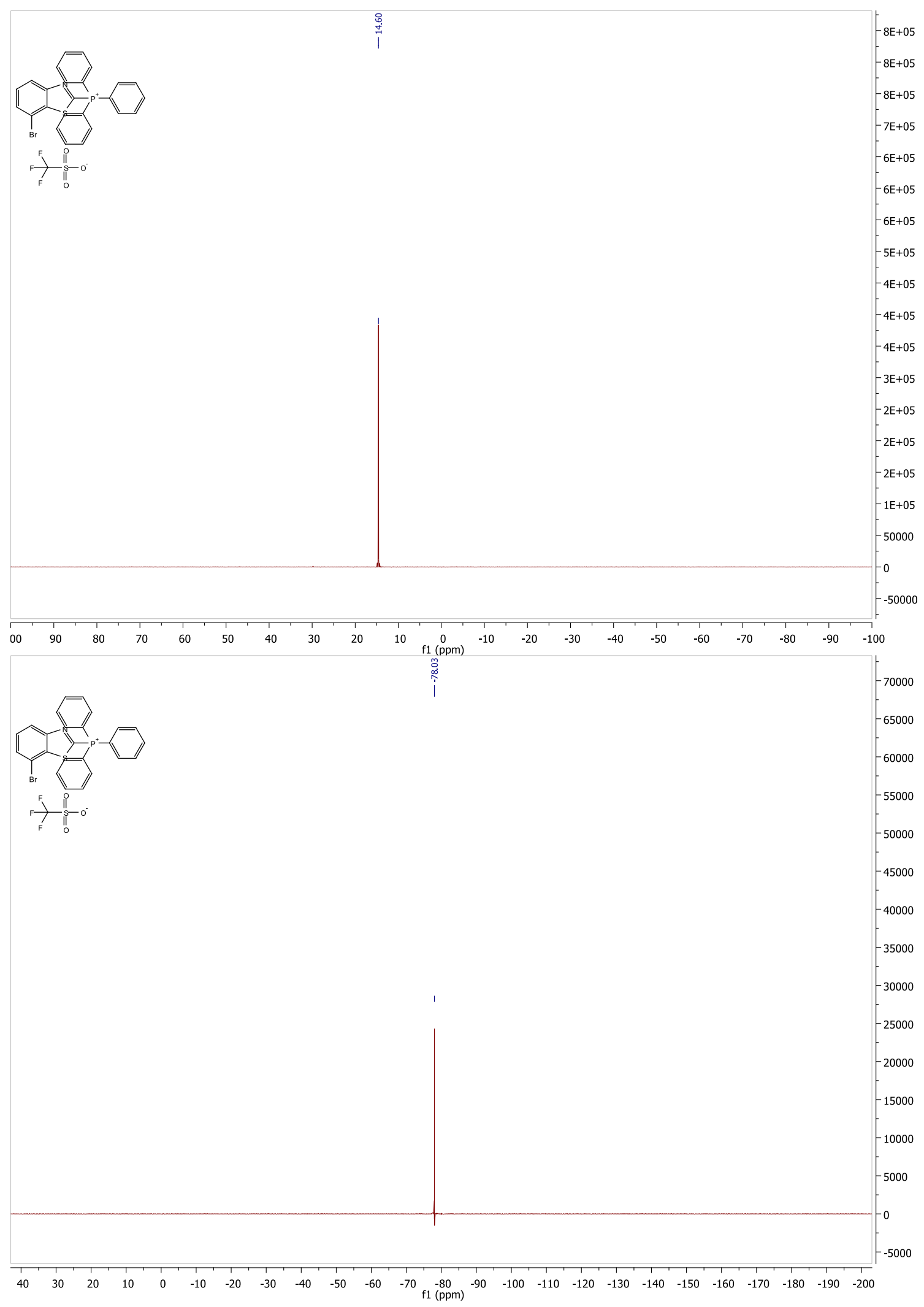


(5-bromobenzo[d]thiazol-2-yl)triphenylphosphonium trifluoromethanesulfonate (2f)

${ }^{1} \mathrm{H}$ NMR $(400 \mathrm{MHz}, \mathrm{CDCl} 3),{ }^{13} \mathrm{C} \mathrm{NMR}(101 \mathrm{MHz}, \mathrm{CDCl} 3),{ }^{19} \mathrm{~F} \mathrm{NMR}(377 \mathrm{MHz}, \mathrm{CDCl} 3)$ and ${ }^{31} \mathrm{P} \mathrm{NMR}(162 \mathrm{MHz}, \mathrm{CDCl} 3)$

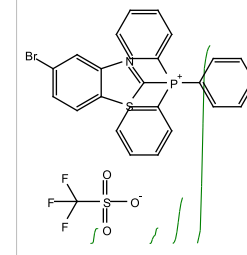

40000

35000

30000

25000

.
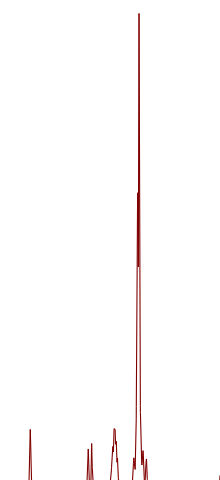

$-20000$

15000

10000

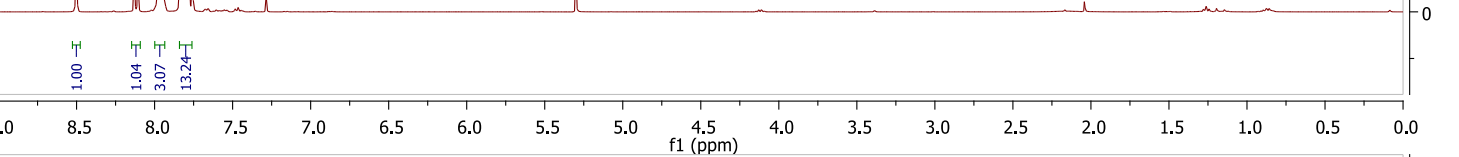

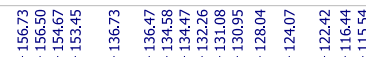
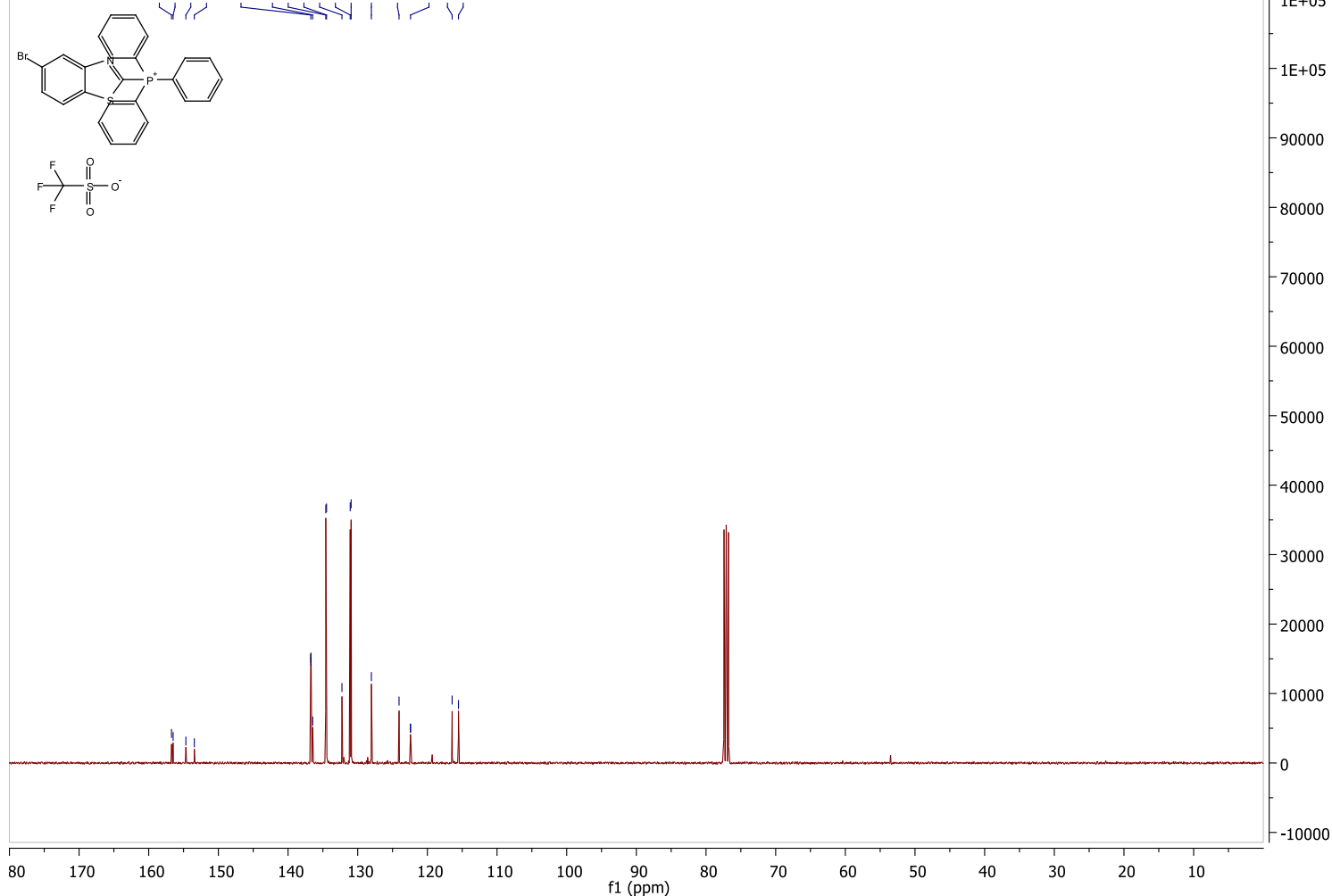


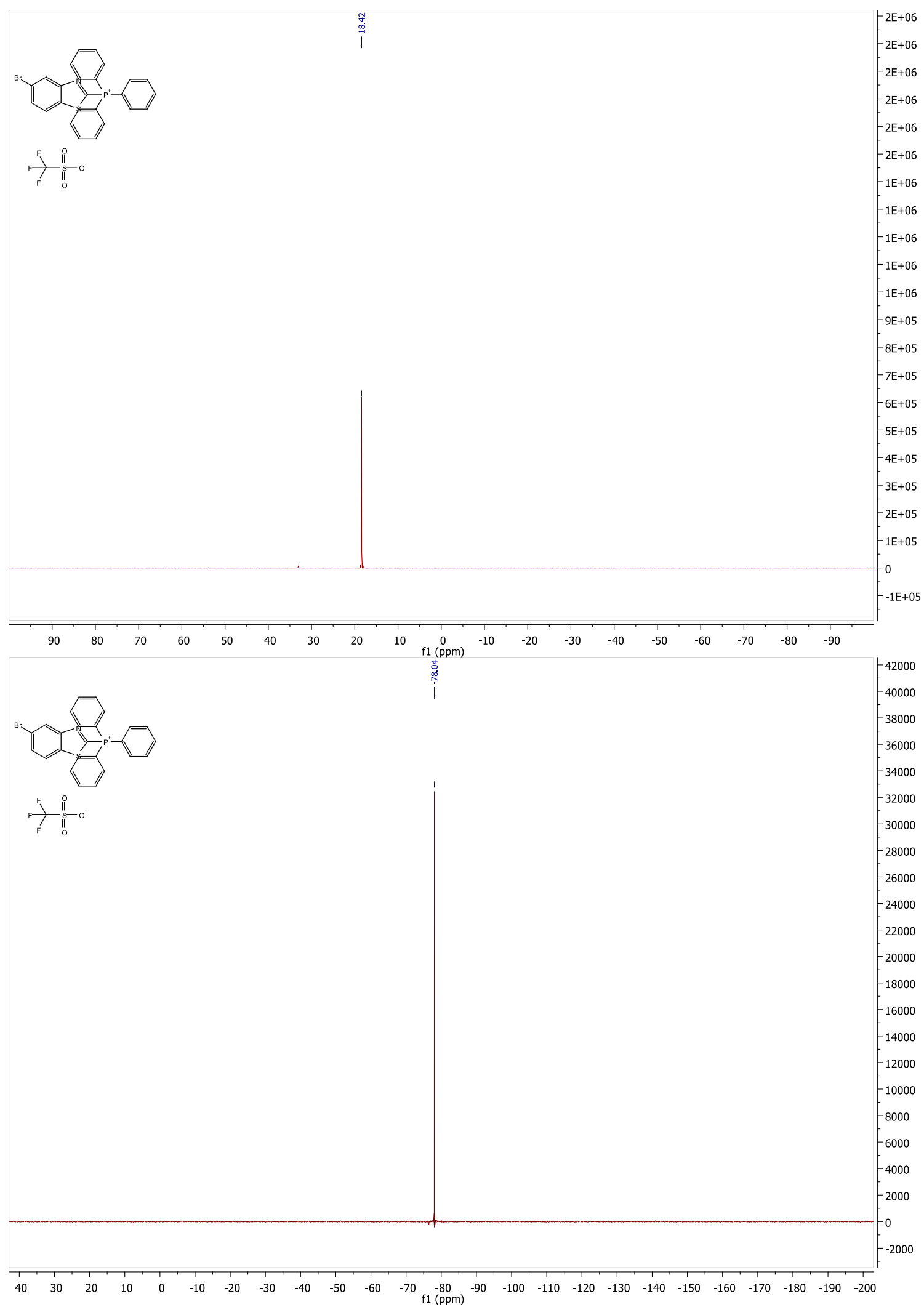


(6-chlorobenzo[d]thiazol-2-yl)triphenylphosphonium trifluoromethanesulfonate (2g)

${ }^{1} \mathrm{H}$ NMR (400 MHz, CDCl3), ${ }^{13} \mathrm{C} \mathrm{NMR}(101 \mathrm{MHz}, \mathrm{CDCl} 3),{ }^{19} \mathrm{~F} \mathrm{NMR}(377 \mathrm{MHz}, \mathrm{CDCl} 3)$ and ${ }^{31} \mathrm{P} \mathrm{NMR}(162 \mathrm{MHz}, \mathrm{CDCl} 3)$

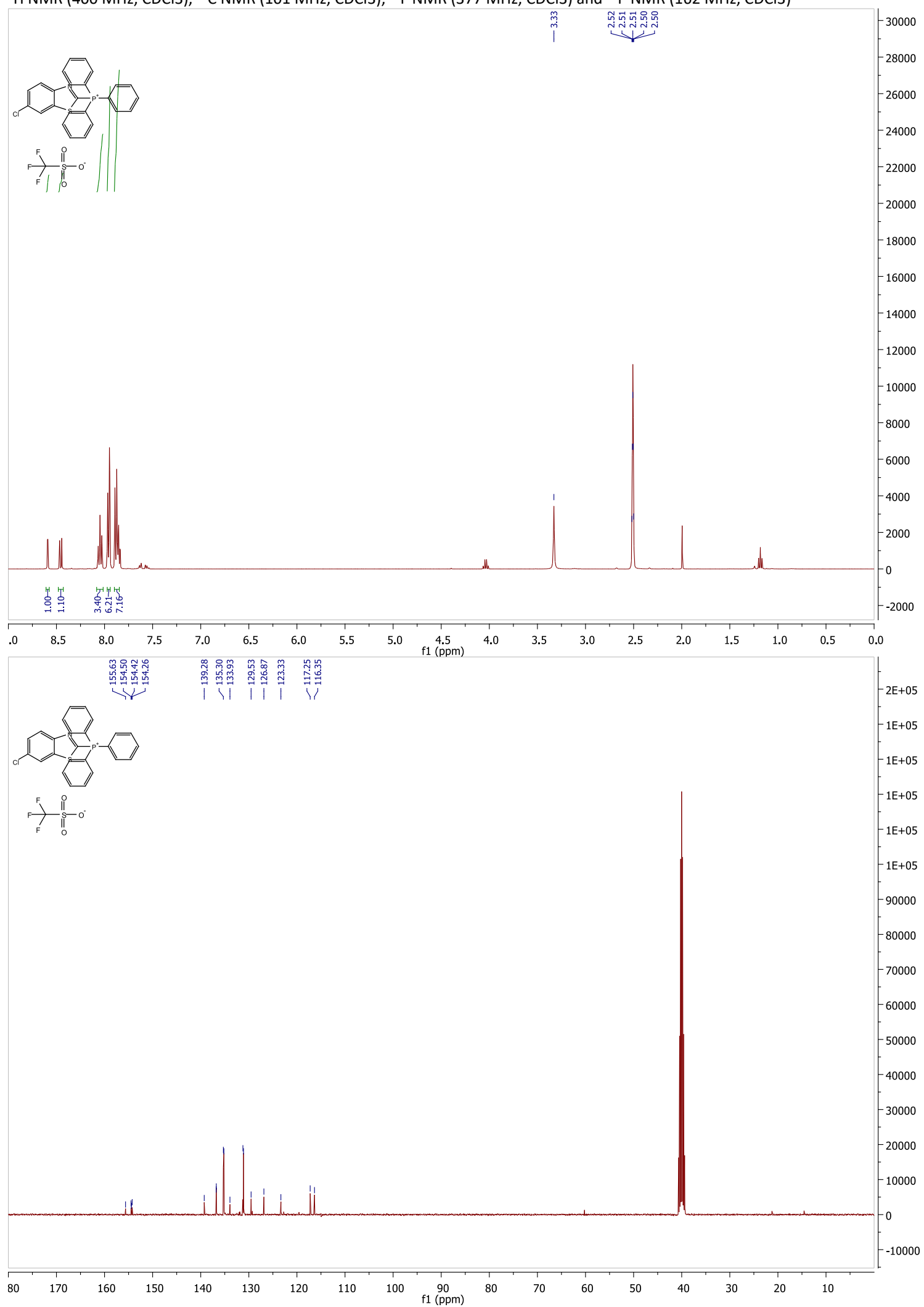




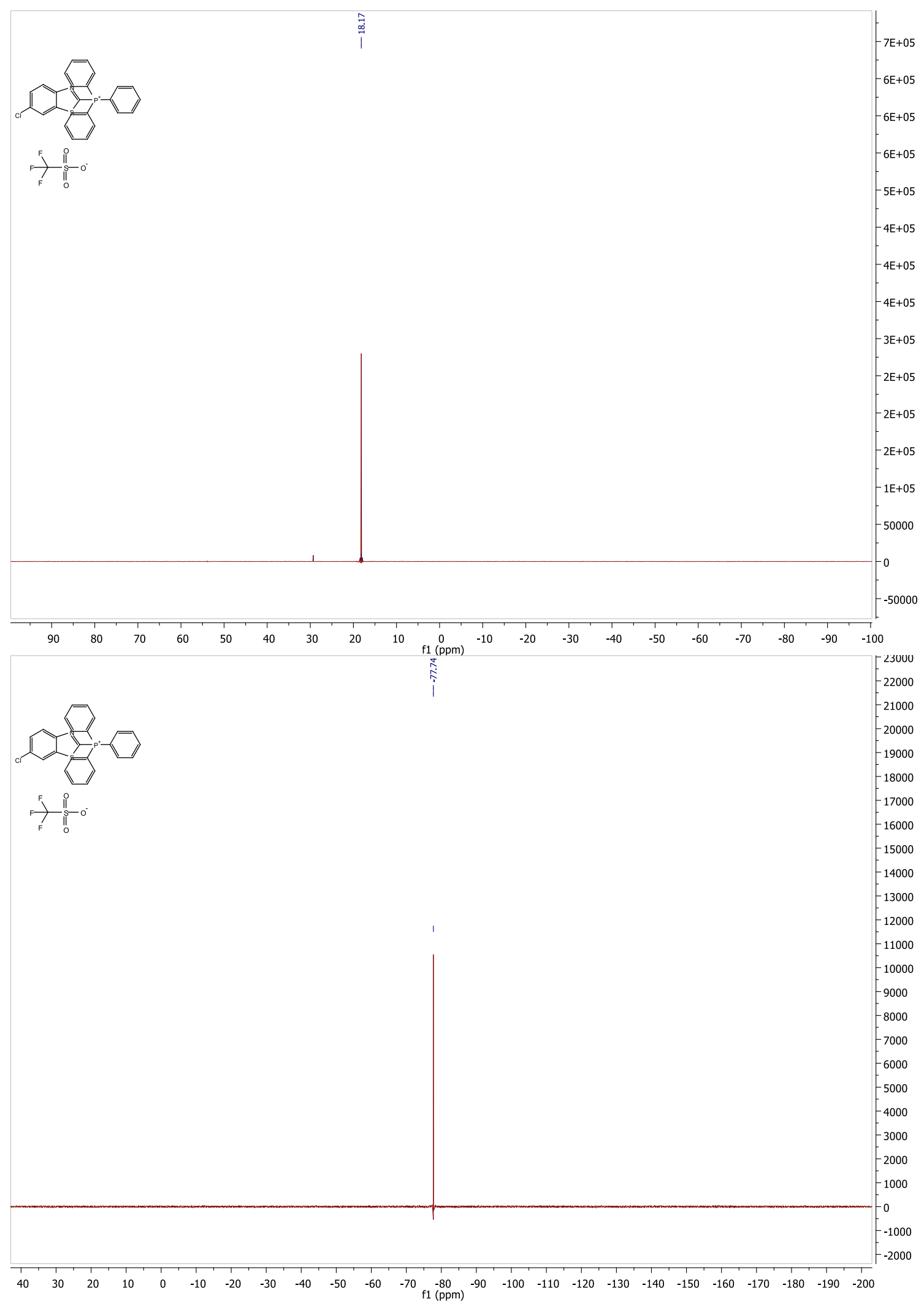


(5-cyanobenzo[d]thiazol-2-yl)triphenylphosphonium trifluoromethanesulfonate (2h)

${ }^{1} \mathrm{H}$ NMR (400 MHz, CDCl3), ${ }^{13} \mathrm{C} N M R(101 \mathrm{MHz}, \mathrm{CDCl} 3),{ }^{19} \mathrm{~F} \mathrm{NMR}(377 \mathrm{MHz}, \mathrm{CDCl} 3)$ and ${ }^{31} \mathrm{P} \mathrm{NMR} \mathrm{(162} \mathrm{MHz,} \mathrm{CDCl3)}$

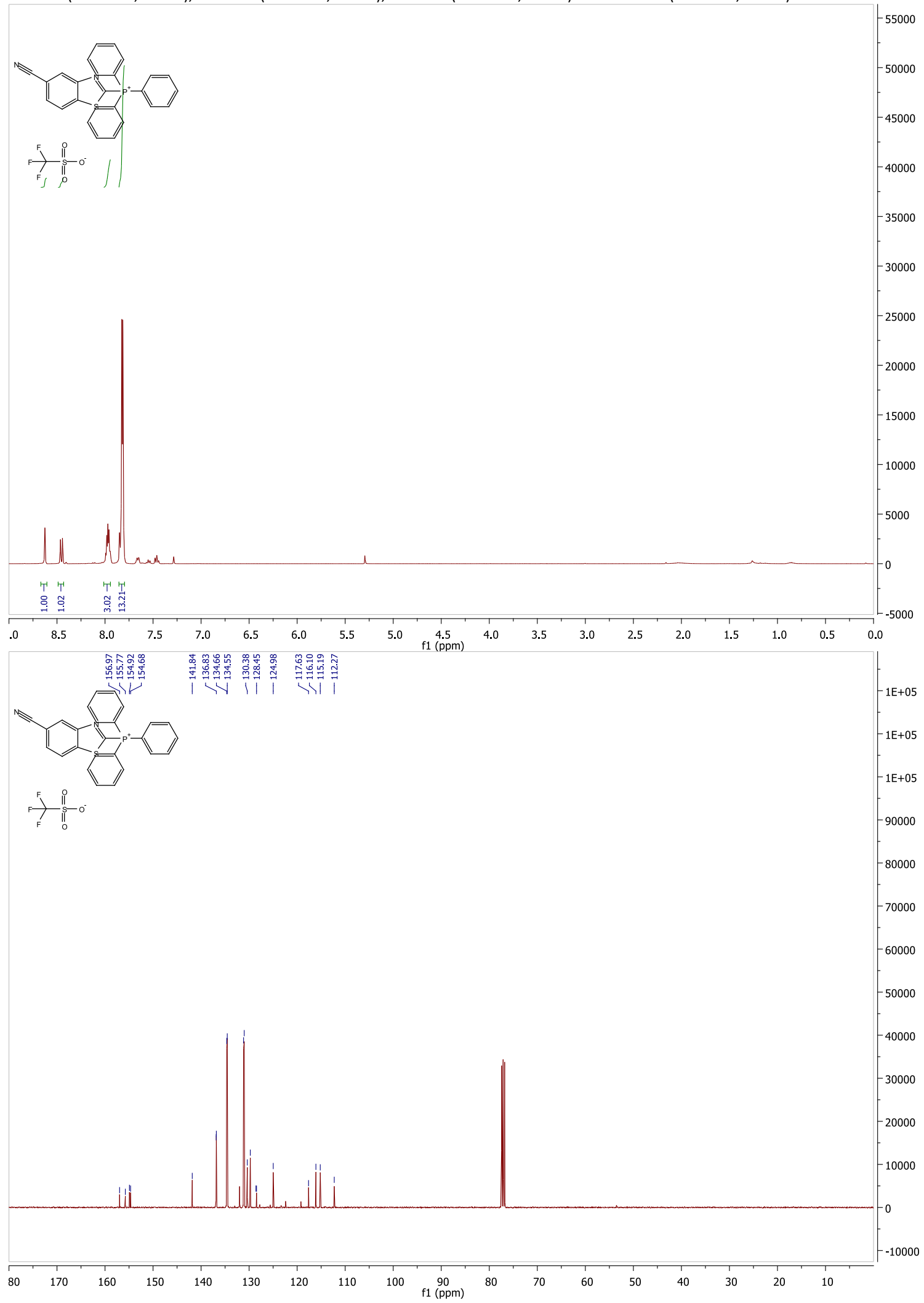




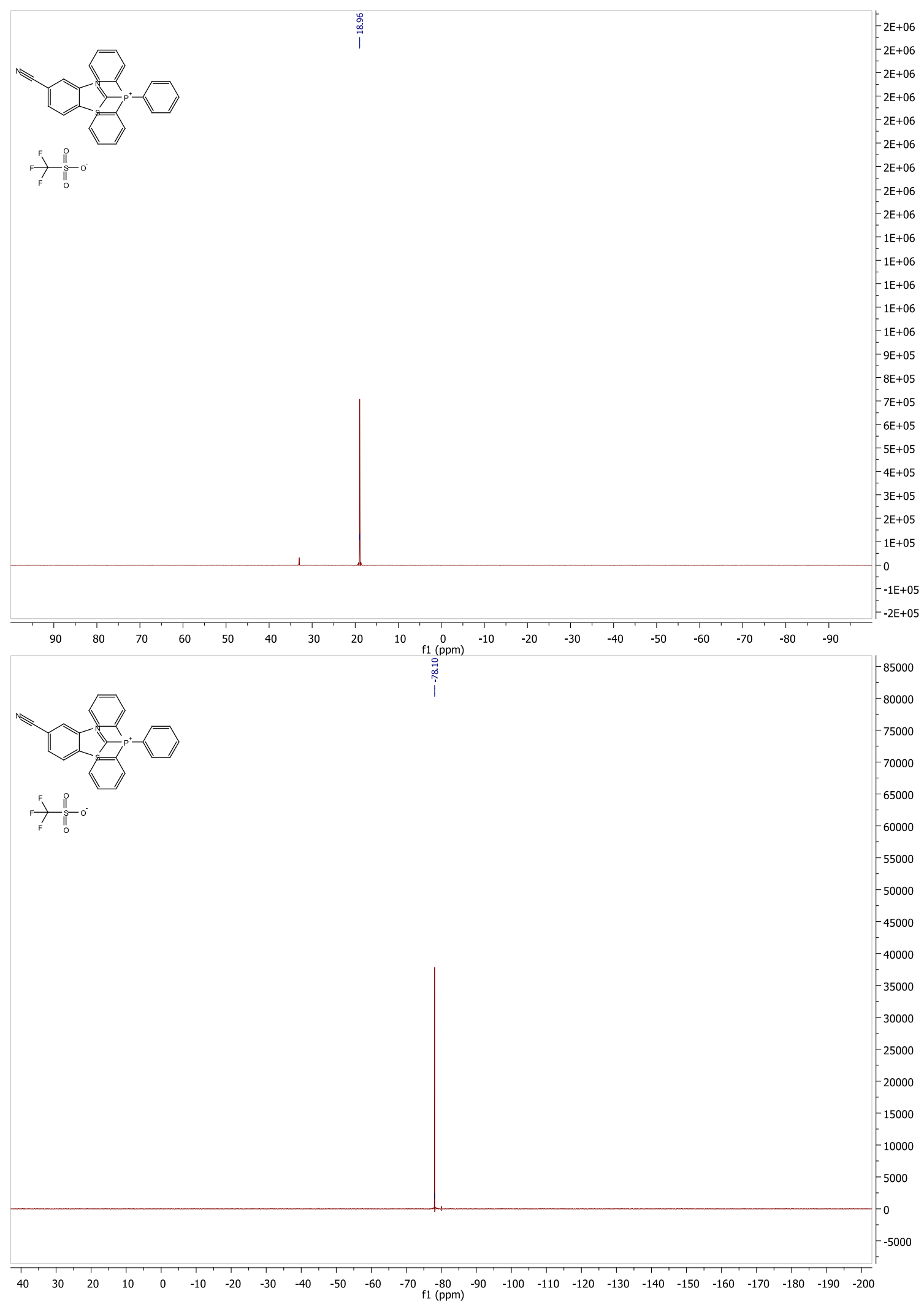


(6-cyanobenzo[d]thiazol-2-yl)triphenylphosphonium trifluoromethanesulfonate (2i)

${ }^{1} \mathrm{H} \mathrm{NMR}(400 \mathrm{MHz}, \mathrm{CDCl} 3),{ }^{13} \mathrm{C} \mathrm{NMR}(101 \mathrm{MHz}, \mathrm{CDCl} 3),{ }^{19} \mathrm{~F} \mathrm{NMR}(377 \mathrm{MHz}, \mathrm{CDCl} 3)$ and ${ }^{31} \mathrm{P}$ NMR $(162 \mathrm{MHz}, \mathrm{CDCl} 3)$

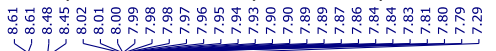
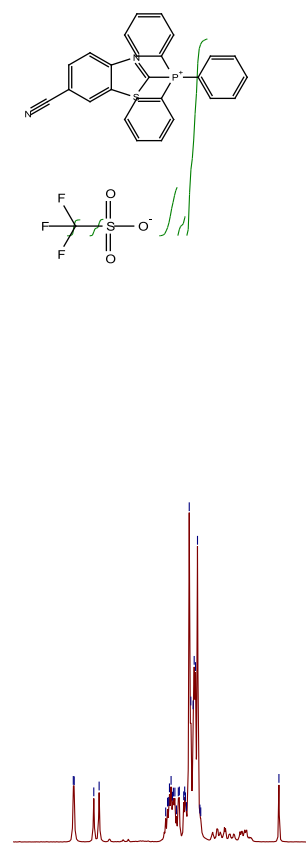

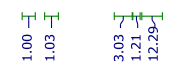
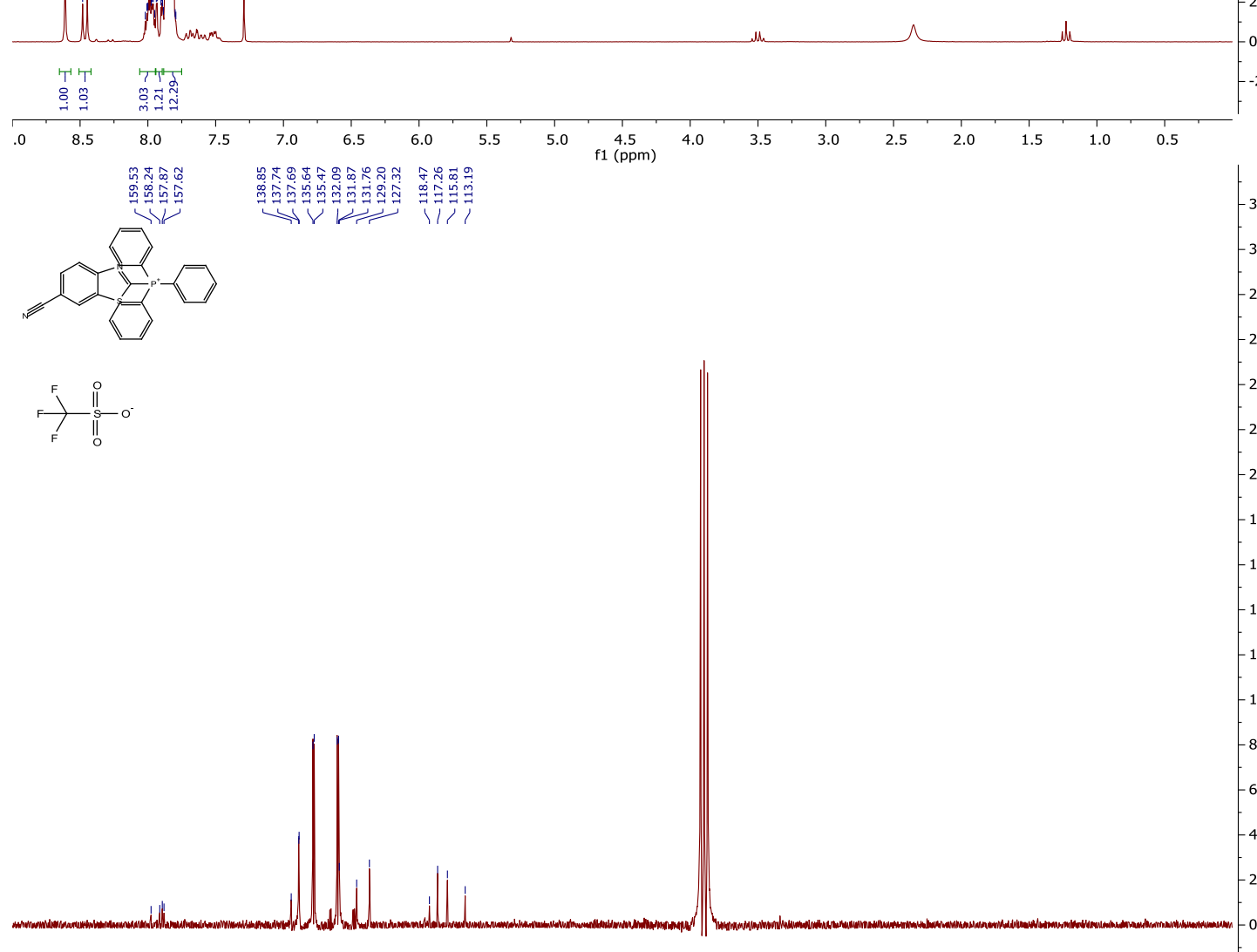

$-32000$

30000

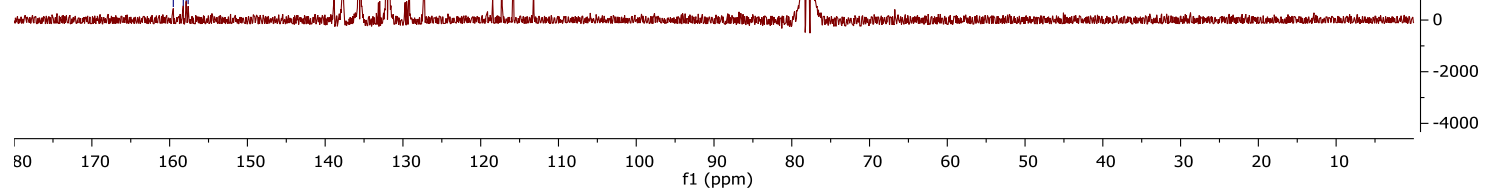




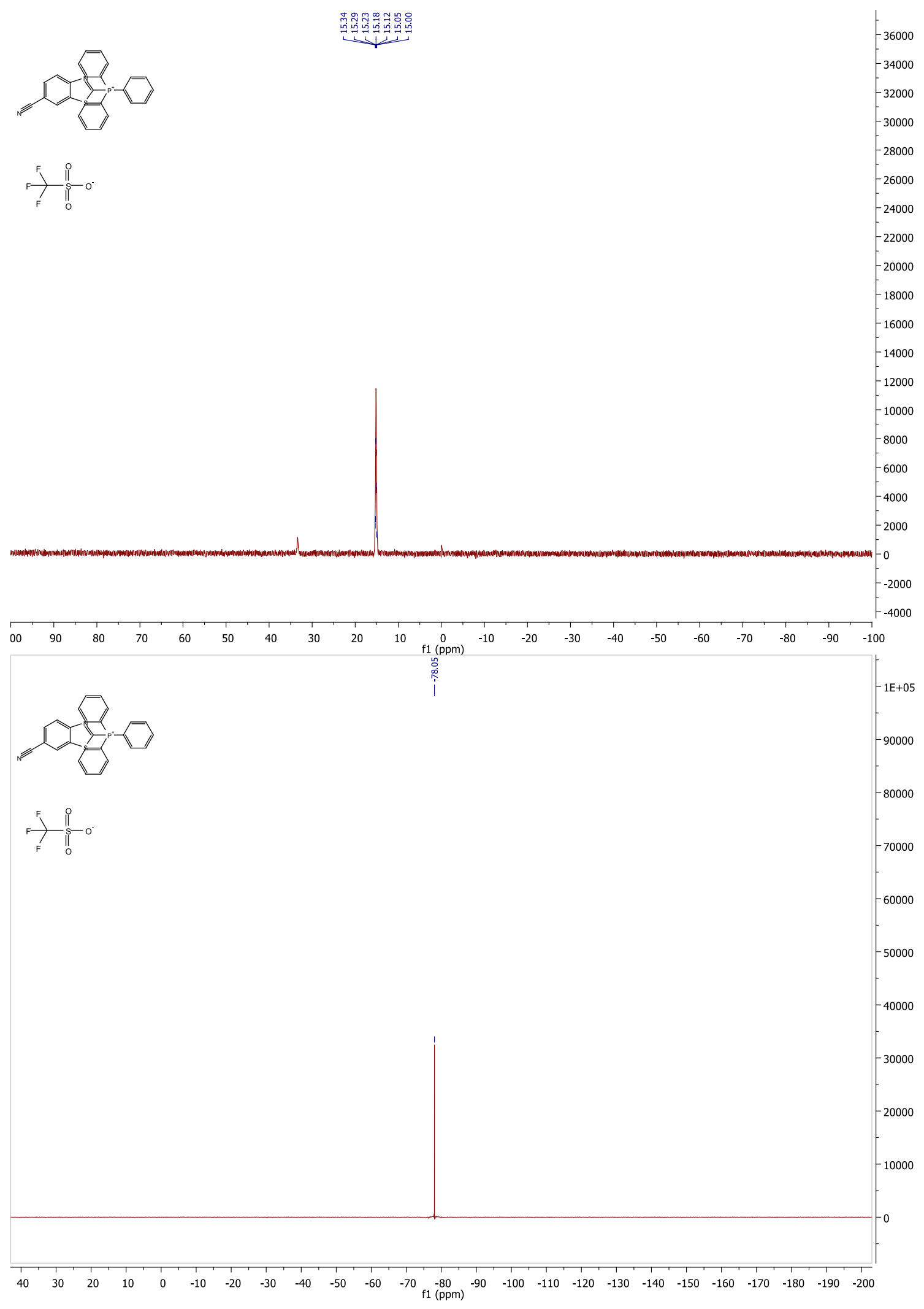


(6-nitrobenzo[d]thiazol-2-yl)triphenylphosphonium trifluoromethanesulfonate (2j)

${ }^{1} \mathrm{H}$ NMR (400 MHz, CDCl3), ${ }^{13} \mathrm{C} N M R(101 \mathrm{MHz}, \mathrm{CDCl} 3),{ }^{19} \mathrm{~F} \mathrm{NMR} \mathrm{(377} \mathrm{MHz,} \mathrm{CDCl3)} \mathrm{and}{ }^{31} \mathrm{P} \mathrm{NMR} \mathrm{(162} \mathrm{MHz,} \mathrm{CDCl3)}$

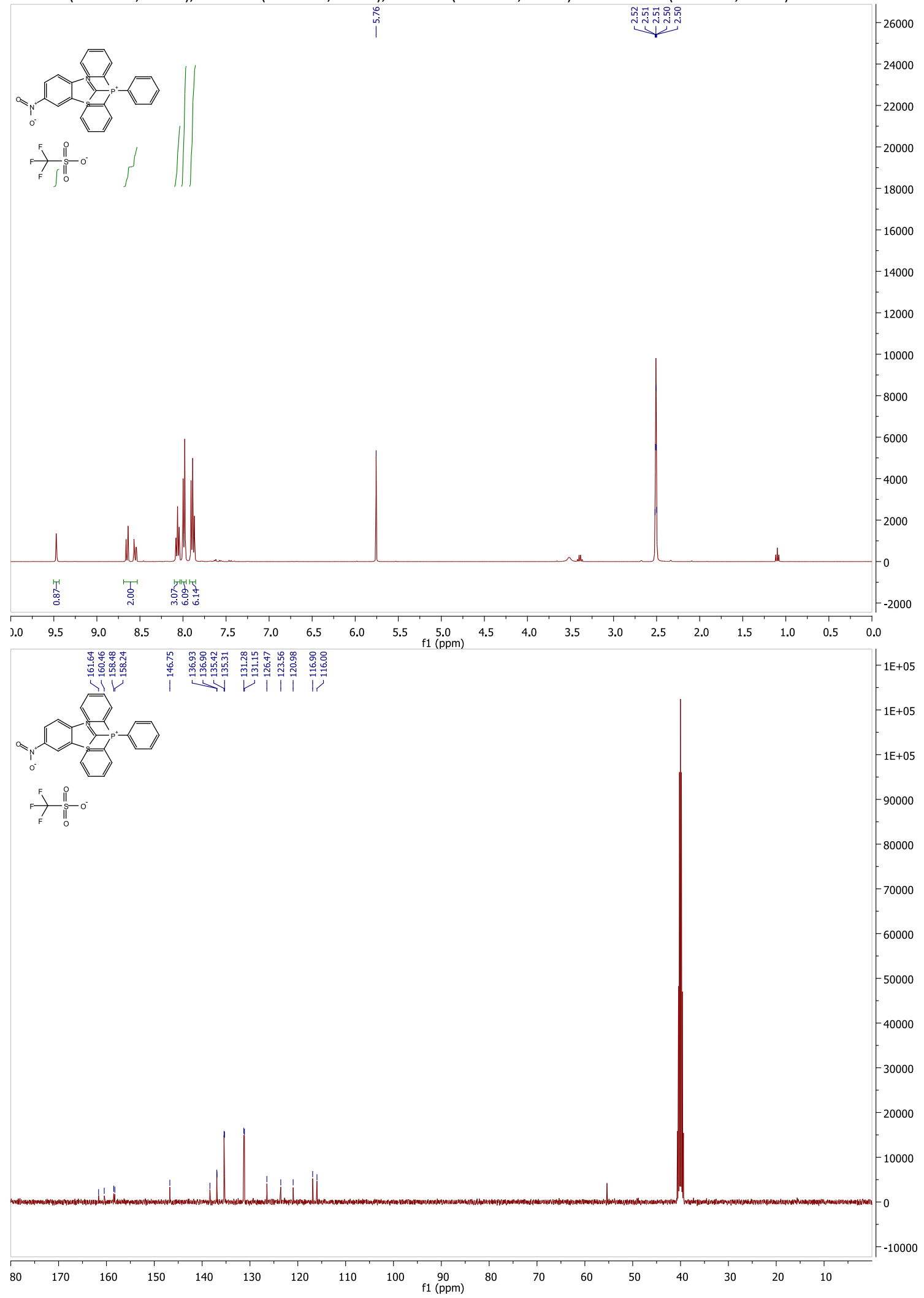




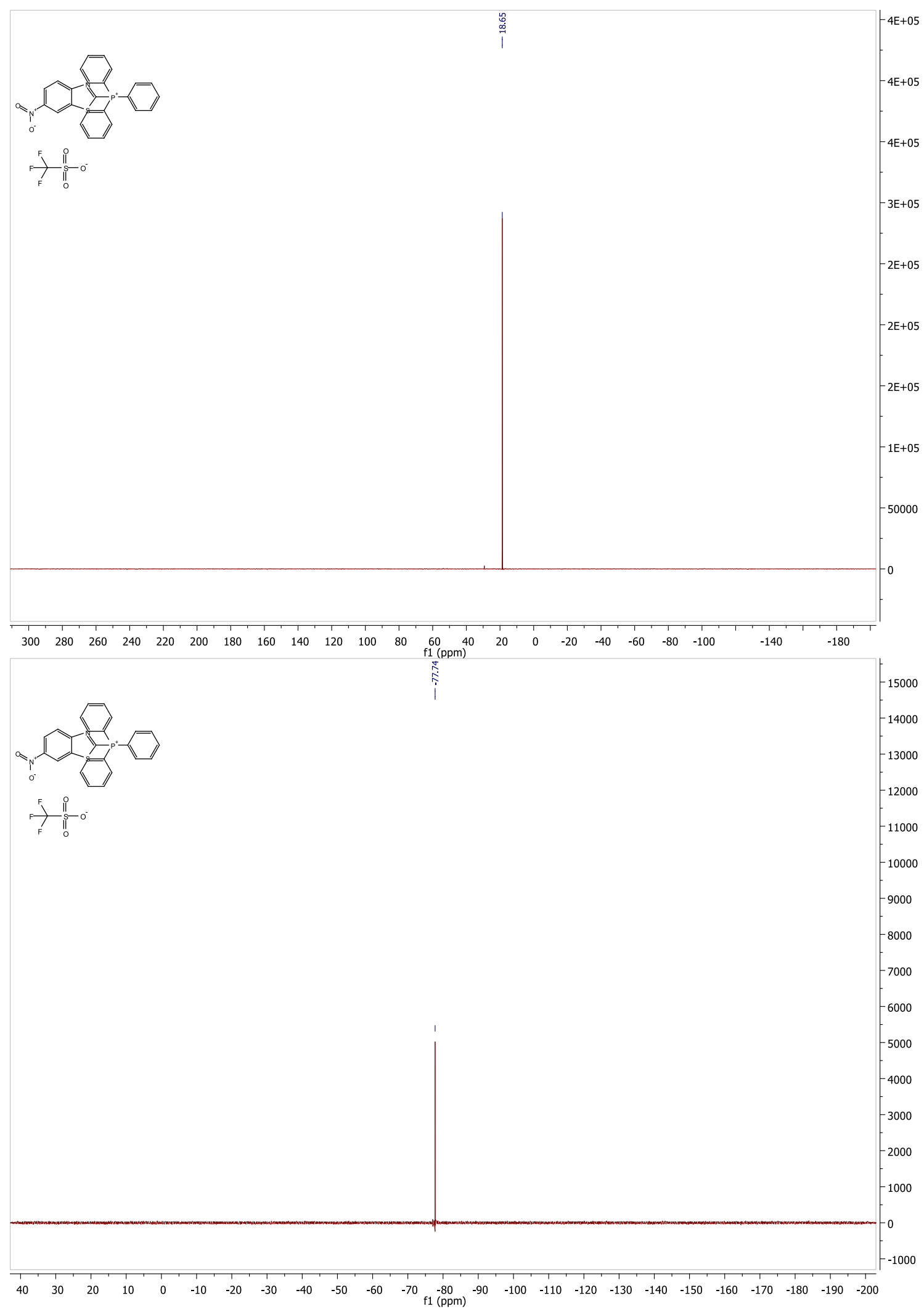




\section{2-(benzyloxy)benzo[d]thiazole (4a)}

${ }^{1} \mathrm{H}$ NMR $(300 \mathrm{MHz}, \mathrm{CDCl} 3)$ and ${ }^{13} \mathrm{C} \mathrm{NMR}(75 \mathrm{MHz}, \mathrm{CDCl} 3)$

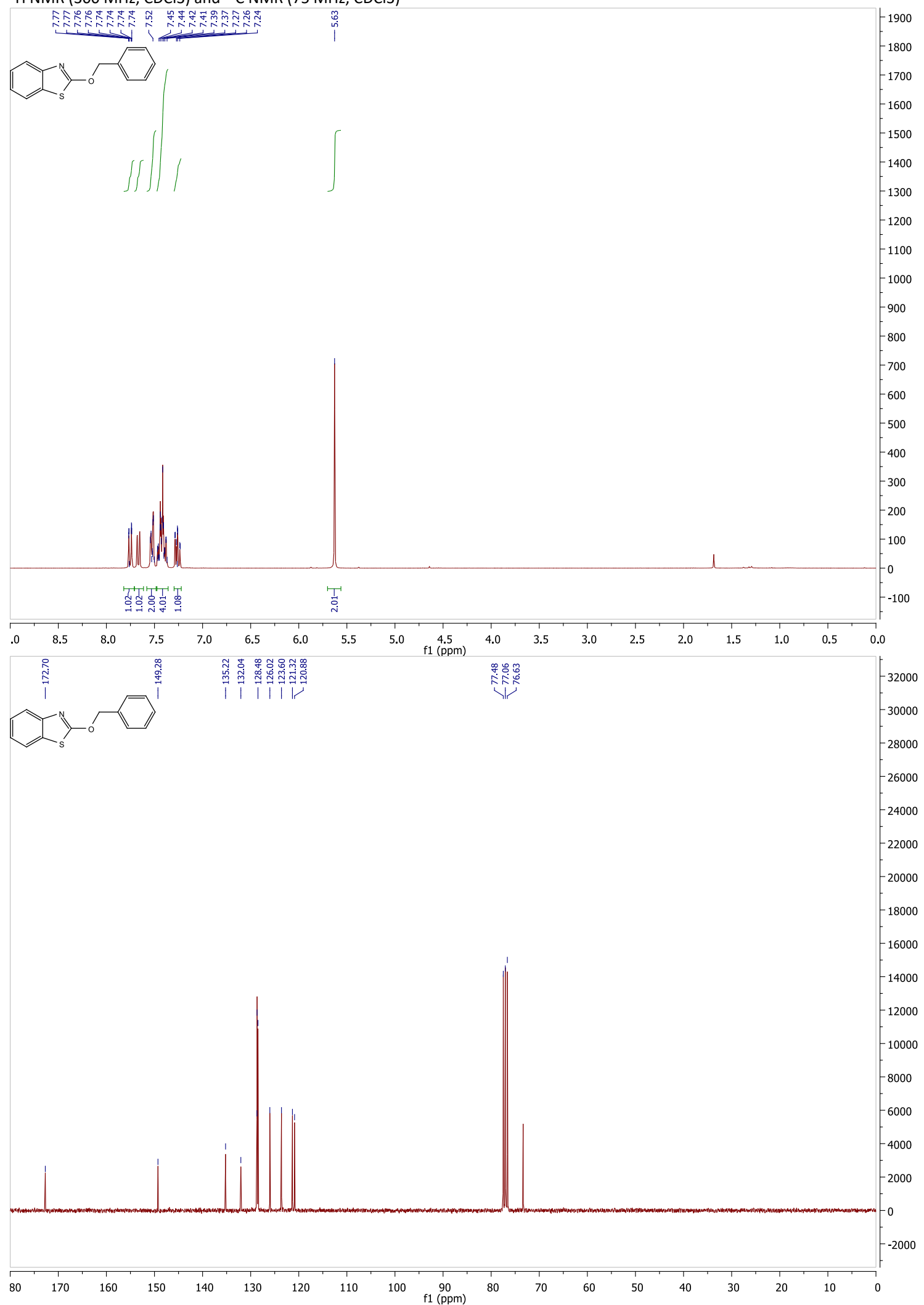


2-((4-bromobenzyl)oxy)benzo[d]thiazole (4b)

${ }^{1} \mathrm{H} \mathrm{NMR}(300 \mathrm{MHz}, \mathrm{CDCl} 3)$ and ${ }^{13} \mathrm{C} \mathrm{NMR}(75 \mathrm{MHz}, \mathrm{CDCl} 3)$

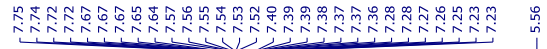

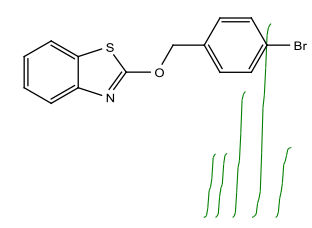


2-([1,1'-biphenyl]-4-ylmethoxy)benzo[d]thiazole (4c)

${ }^{1} \mathrm{H}$ NMR $(300 \mathrm{MHz}, \mathrm{CDCl} 3)$ and ${ }^{13} \mathrm{C} \mathrm{NMR}(75 \mathrm{MHz}, \mathrm{CDCl} 3)$

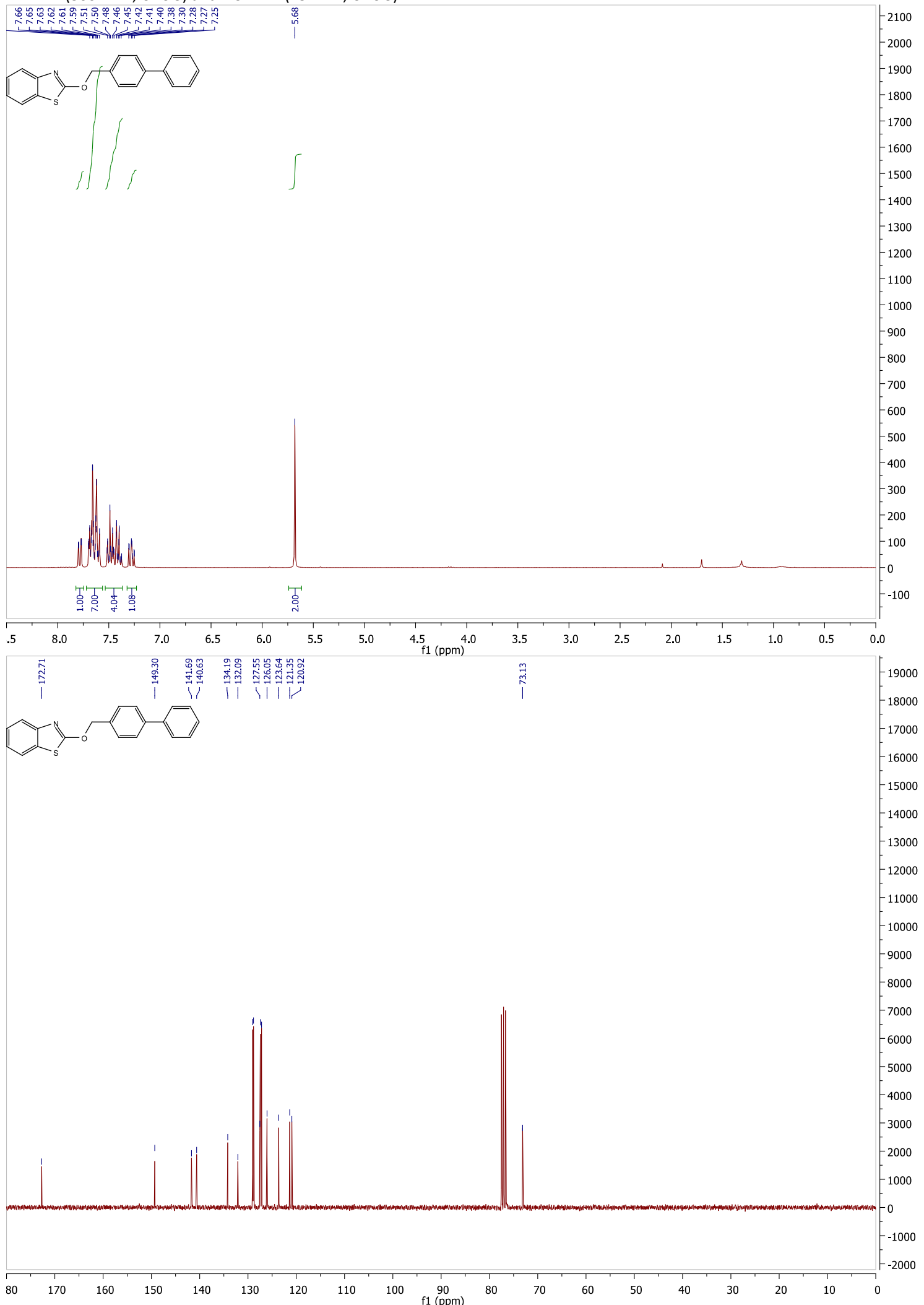




\section{2-(3-phenylpropoxy)benzo[d]thiazole (4d)}

${ }^{1} \mathrm{H}$ NMR $(300 \mathrm{MHz}, \mathrm{CDCl} 3)$ and ${ }^{13} \mathrm{C} \mathrm{NMR}(75 \mathrm{MHz}, \mathrm{CDCl} 3)$

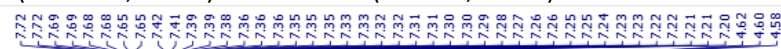
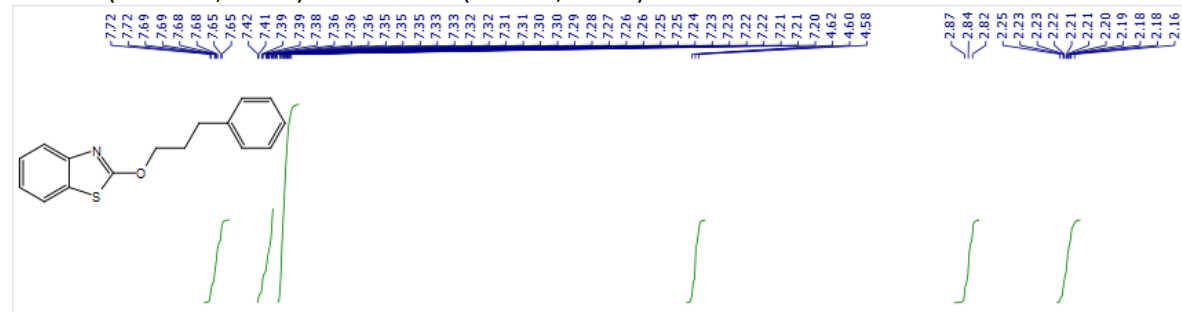

$-1100$

1000
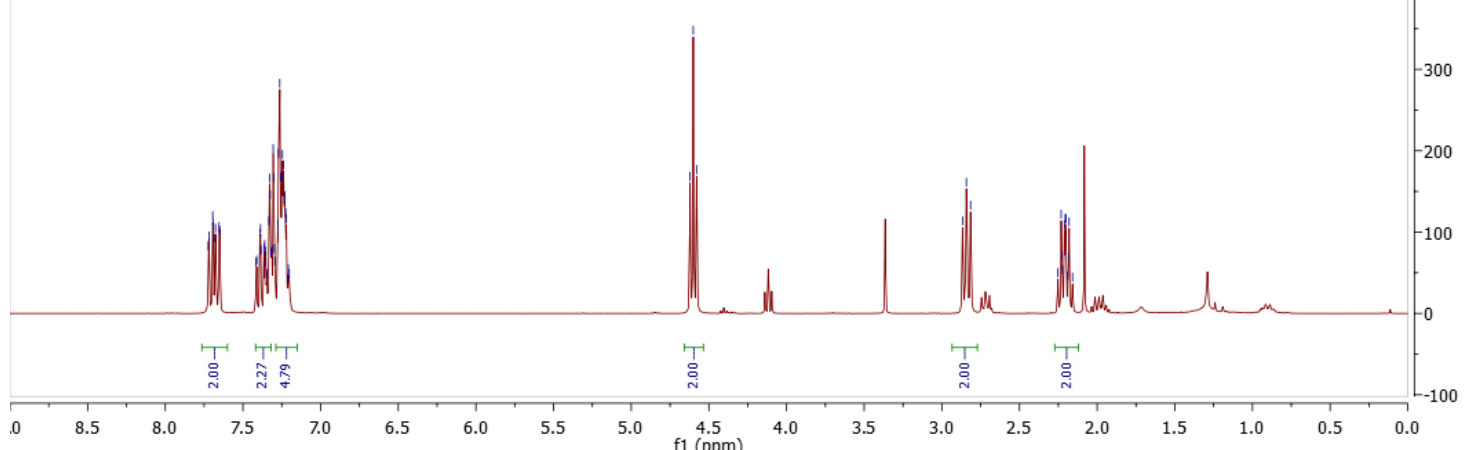

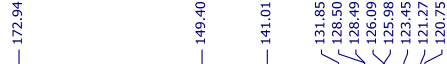

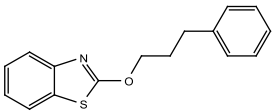

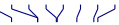

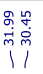

30000

25000

20000

15000

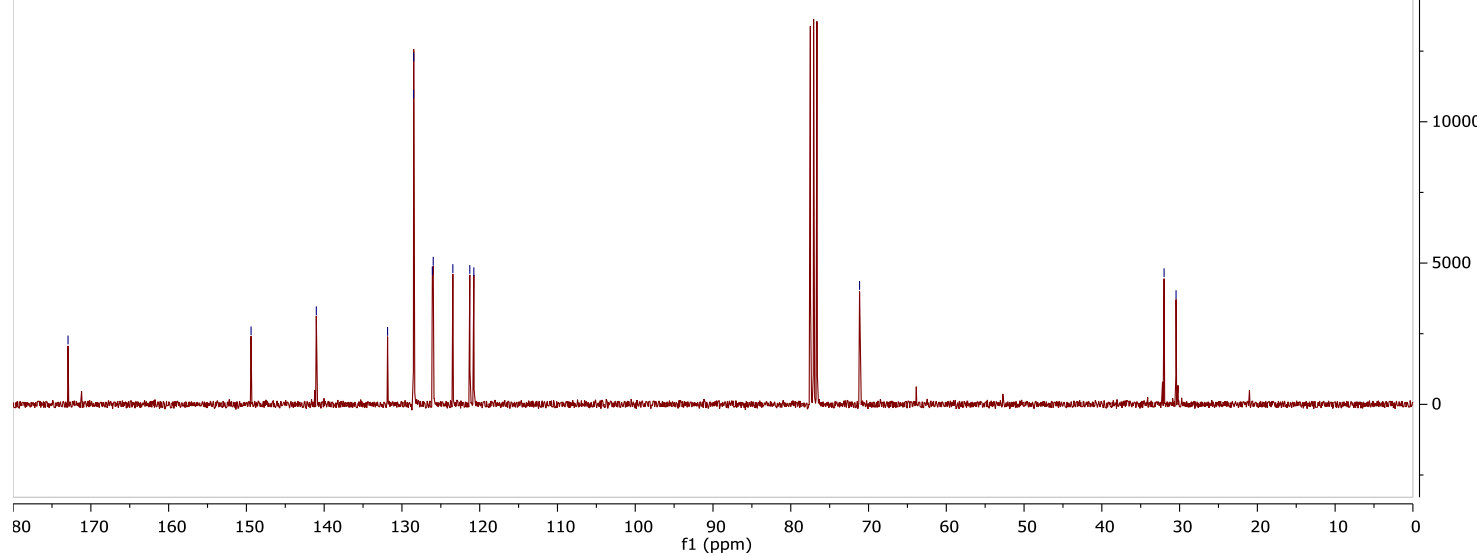


2-(2,2,2-trifluoroethoxy)benzo[d]thiazole (4e)

${ }^{1} \mathrm{H} \mathrm{NMR}\left(300 \mathrm{MHz}, \mathrm{CDCl}_{3}\right),{ }^{13} \mathrm{C} \mathrm{NMR}\left(75 \mathrm{MHz}, \mathrm{CDCl}_{3}\right)$ and ${ }^{19} \mathrm{~F} \mathrm{NMR}\left(377 \mathrm{MHz}, \mathrm{CDCl}_{3}\right)$

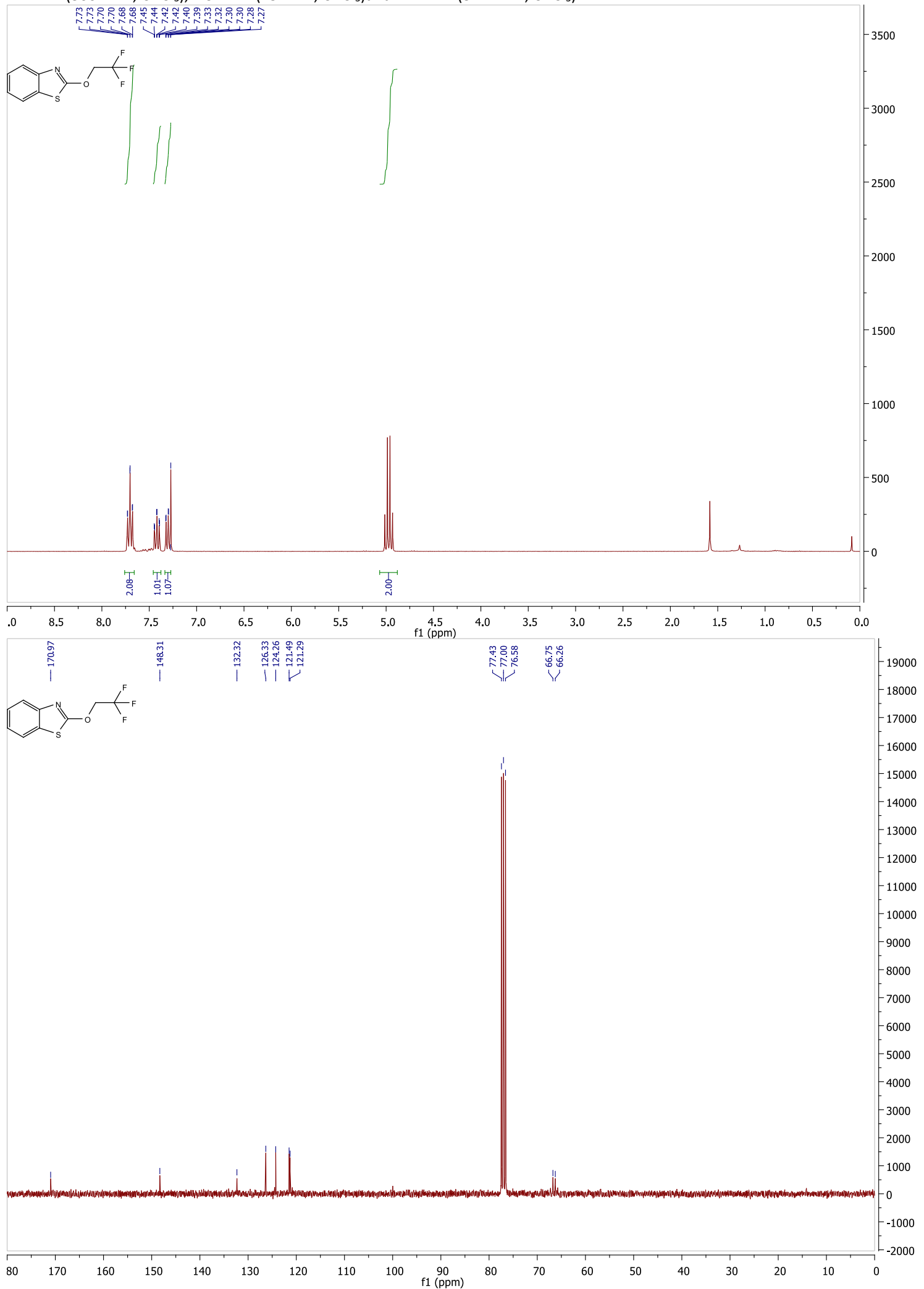




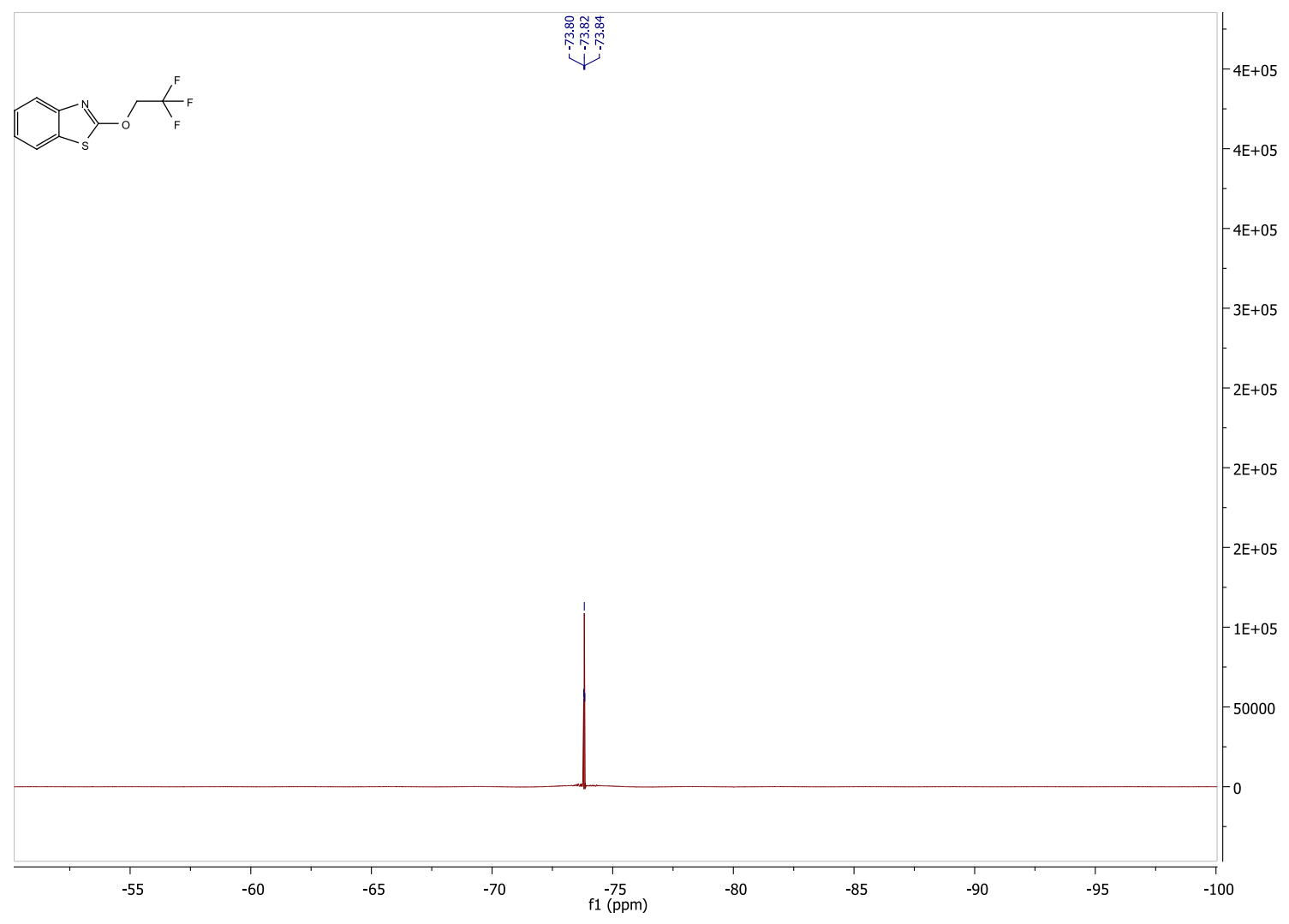




\section{2-(cinnamyloxy)benzo[d]thiazole (4f)}

${ }^{1} \mathrm{H} \mathrm{NMR}\left(300 \mathrm{MHz}, \mathrm{CDCl}_{3}\right)$ and ${ }^{13} \mathrm{C} \mathrm{NMR}\left(75 \mathrm{MHz}, \mathrm{CDCl}_{3}\right)$

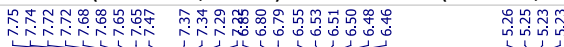

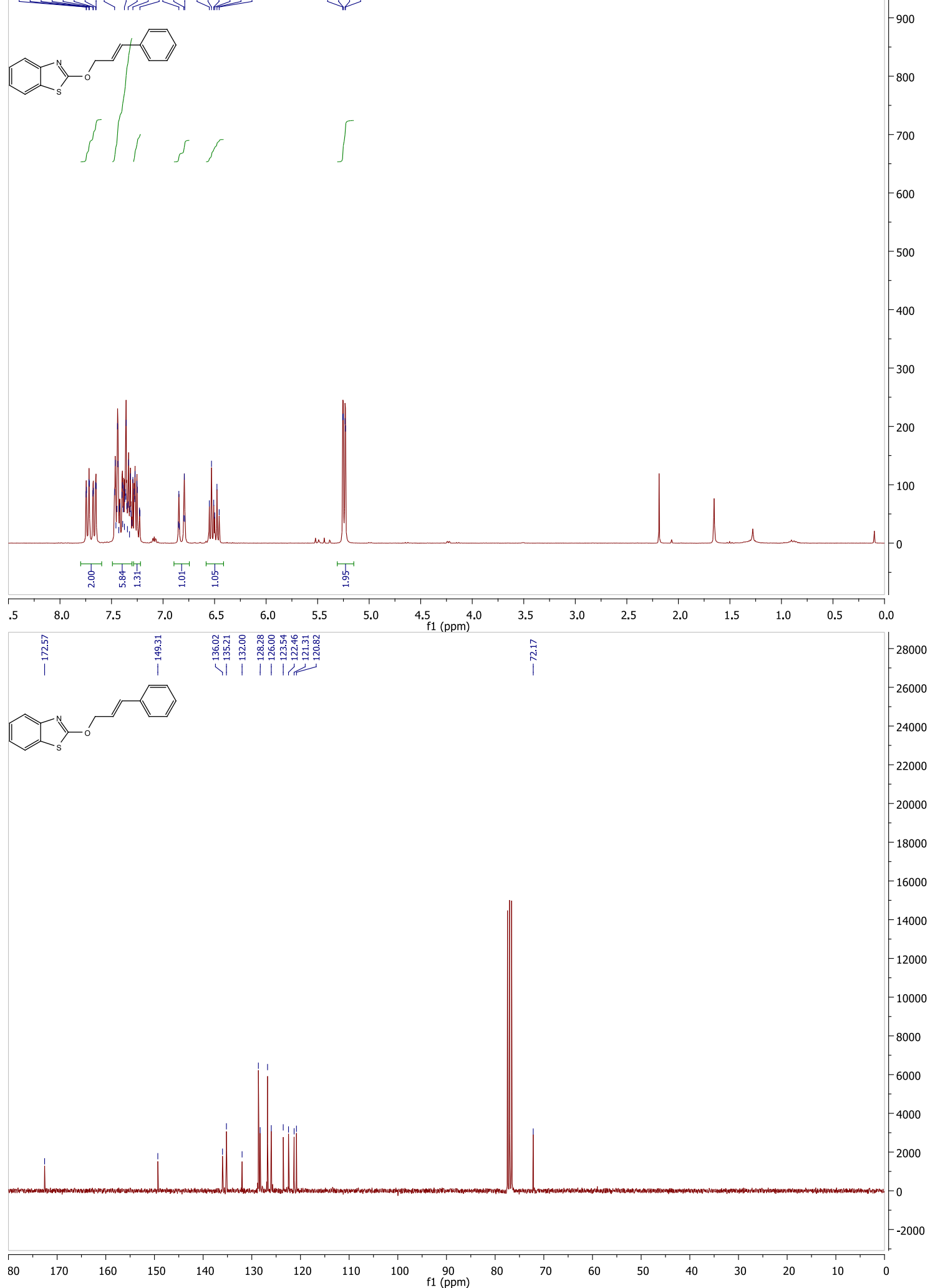


2-(but-2-yn-1-yloxy)benzo[d]thiazole (4g)

${ }^{1} \mathrm{H} \mathrm{NMR}\left(300 \mathrm{MHz}, \mathrm{CDCl}_{3}\right)$ and ${ }^{13} \mathrm{C} \mathrm{NMR}\left(75 \mathrm{MHz}, \mathrm{CDCl}_{3}\right)$

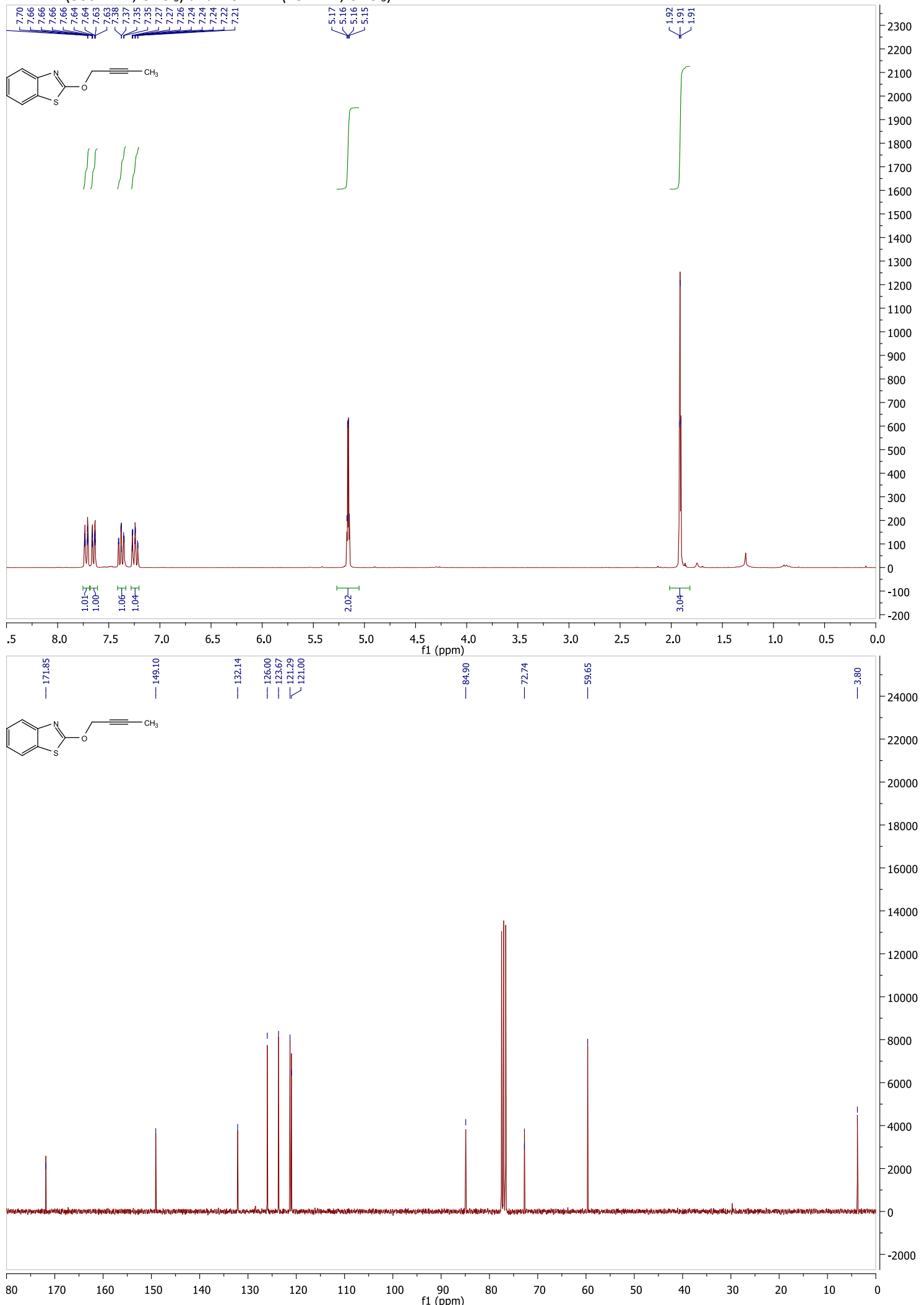


2-(prop-2-yn-1-yloxy)benzo[d]thiazole (4h)

${ }^{1} \mathrm{H} \mathrm{NMR}\left(300 \mathrm{MHz}, \mathrm{CDCl}_{3}\right)$ and ${ }^{13} \mathrm{C} \mathrm{NMR}\left(75 \mathrm{MHz}, \mathrm{CDCl}_{3}\right)$

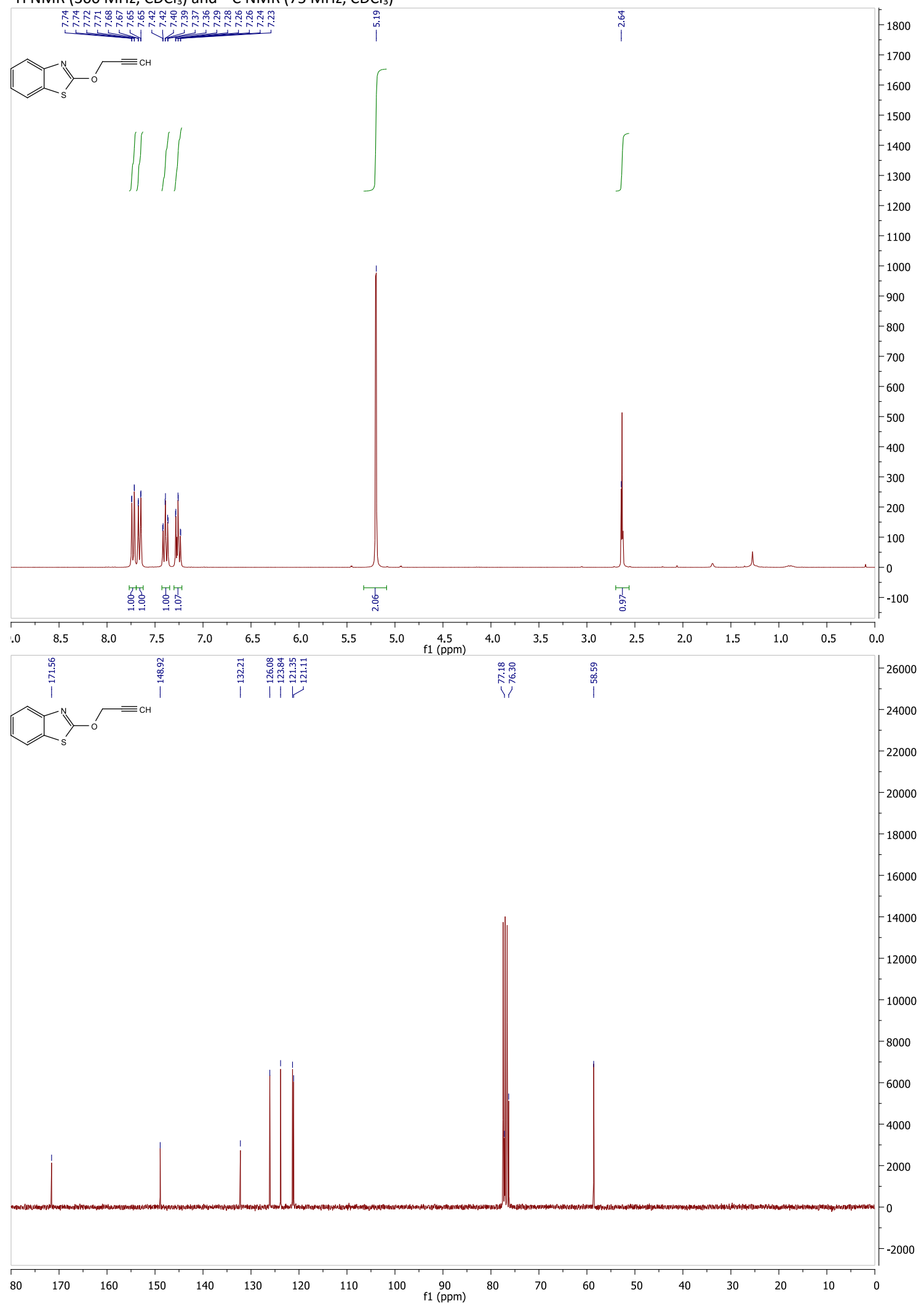




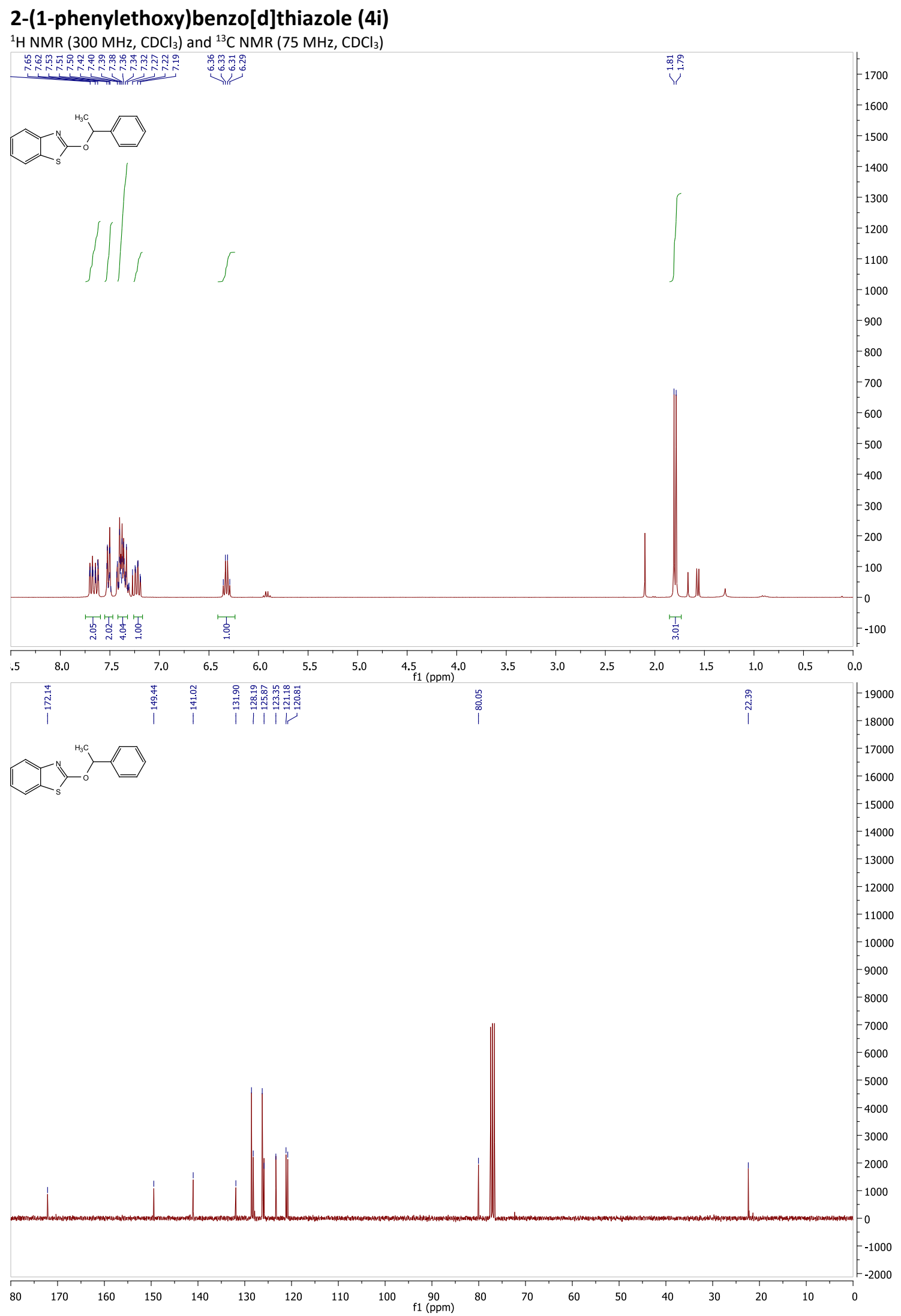


2-(1-phenylpropoxy)benzo[d]thiazole (4j)

${ }^{1} \mathrm{H} \mathrm{NMR}\left(300 \mathrm{MHz}, \mathrm{CDCl}_{3}\right)$ and ${ }^{13} \mathrm{C} \mathrm{NMR}\left(75 \mathrm{MHz}, \mathrm{CDCl}_{3}\right)$

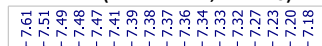

ت⿸广

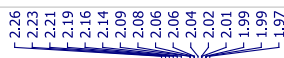

$\sum_{s}^{N}$
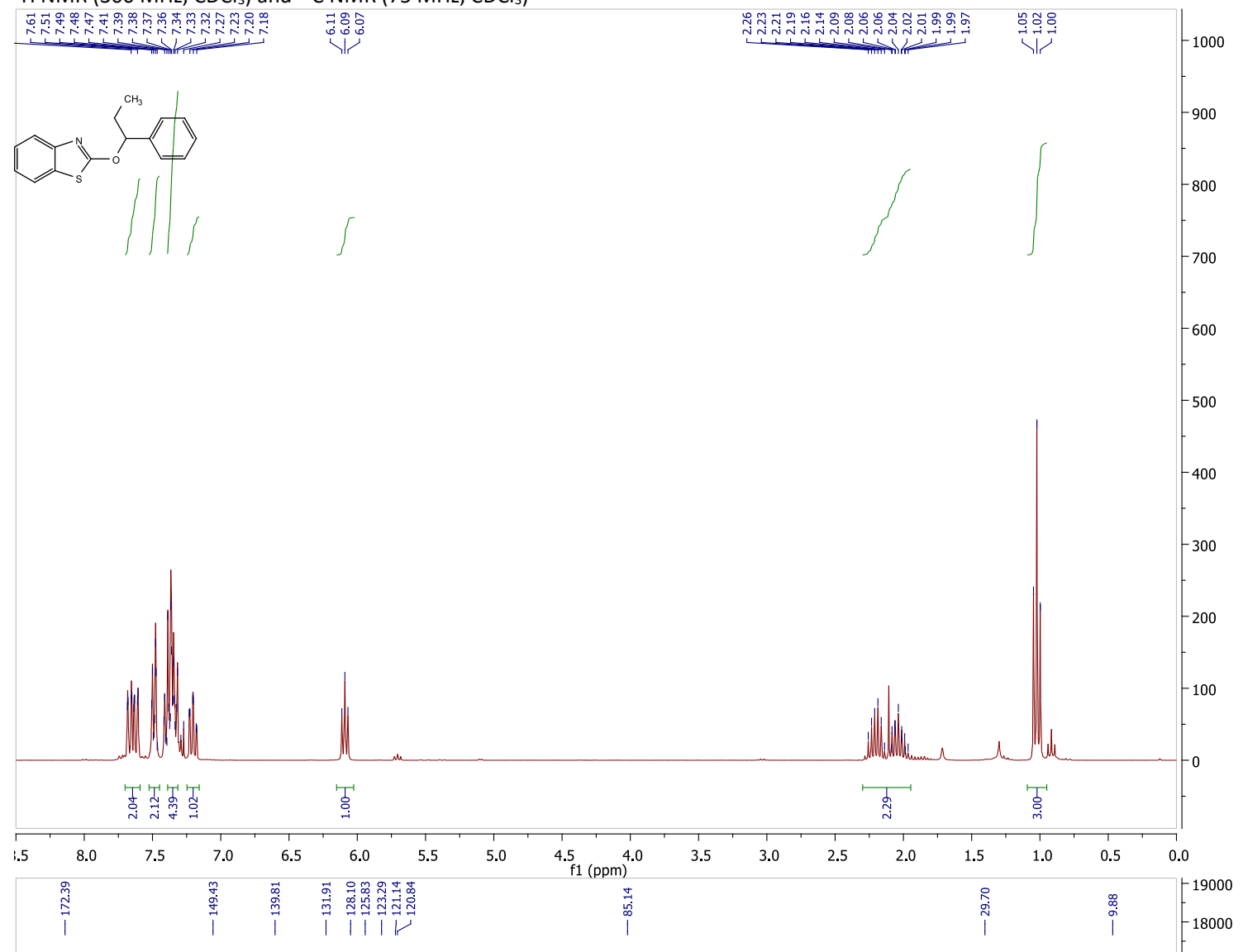<smiles>CCC(Oc1nc2ccccc2s1)c1ccccc1</smiles>
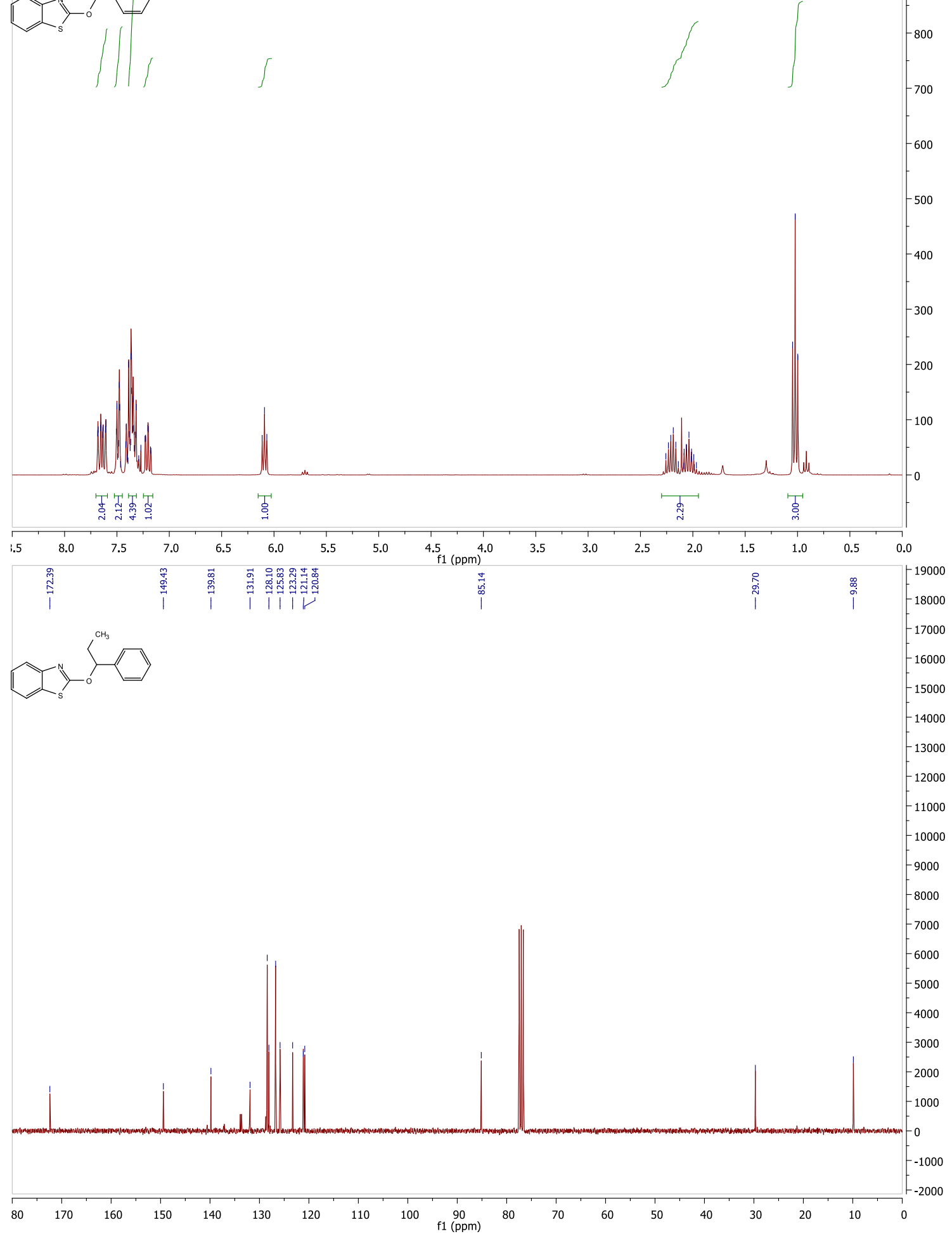
2-(pent-1-yn-3-yloxy)benzo[d]thiazole (4k)

${ }^{1} \mathrm{H}$ NMR $\left(300 \mathrm{MHz}, \mathrm{CDCl}_{3}\right)$ and ${ }^{13} \mathrm{C} \mathrm{NMR}\left(75 \mathrm{MHz}, \mathrm{CDCl}_{3}\right)$

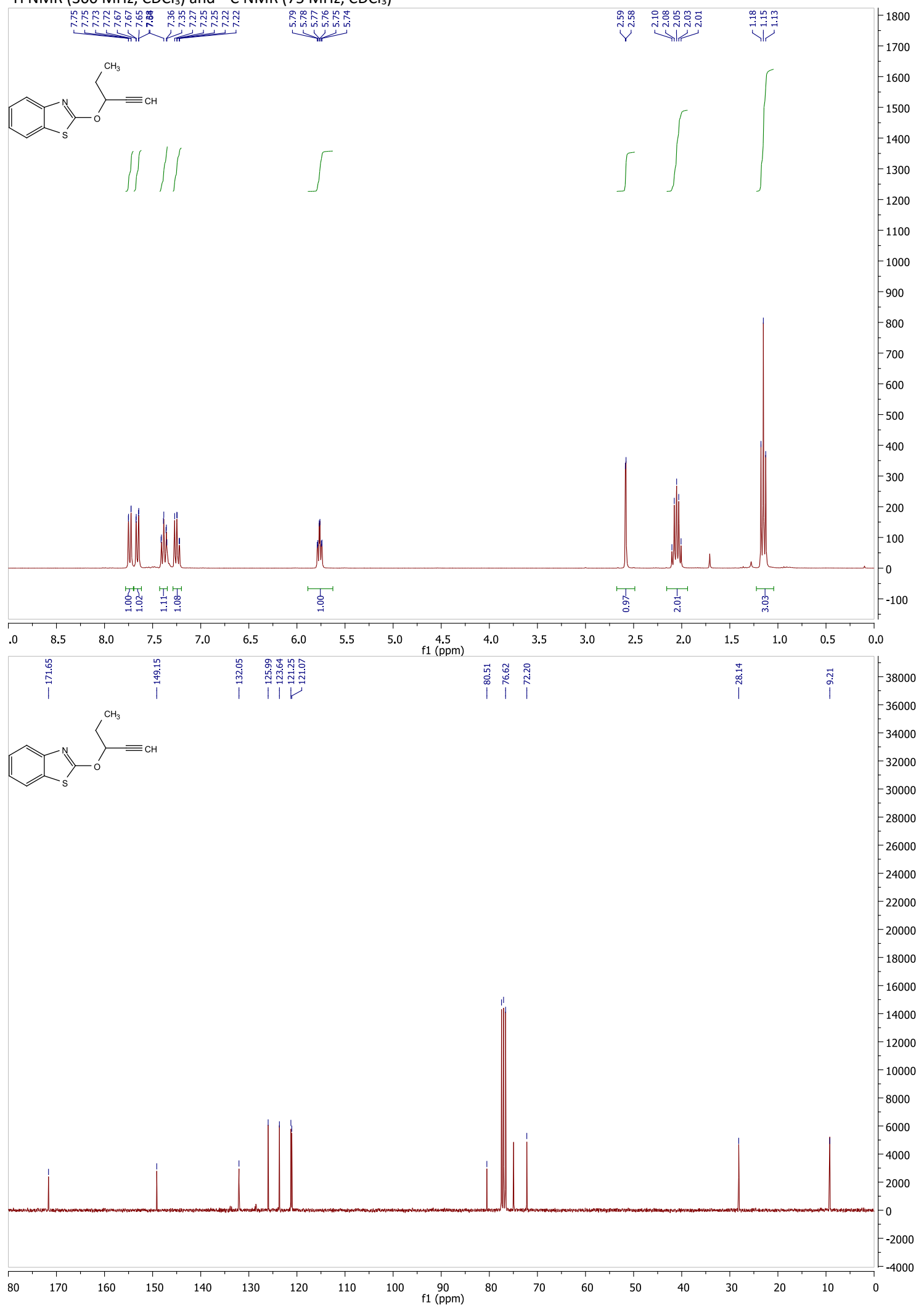




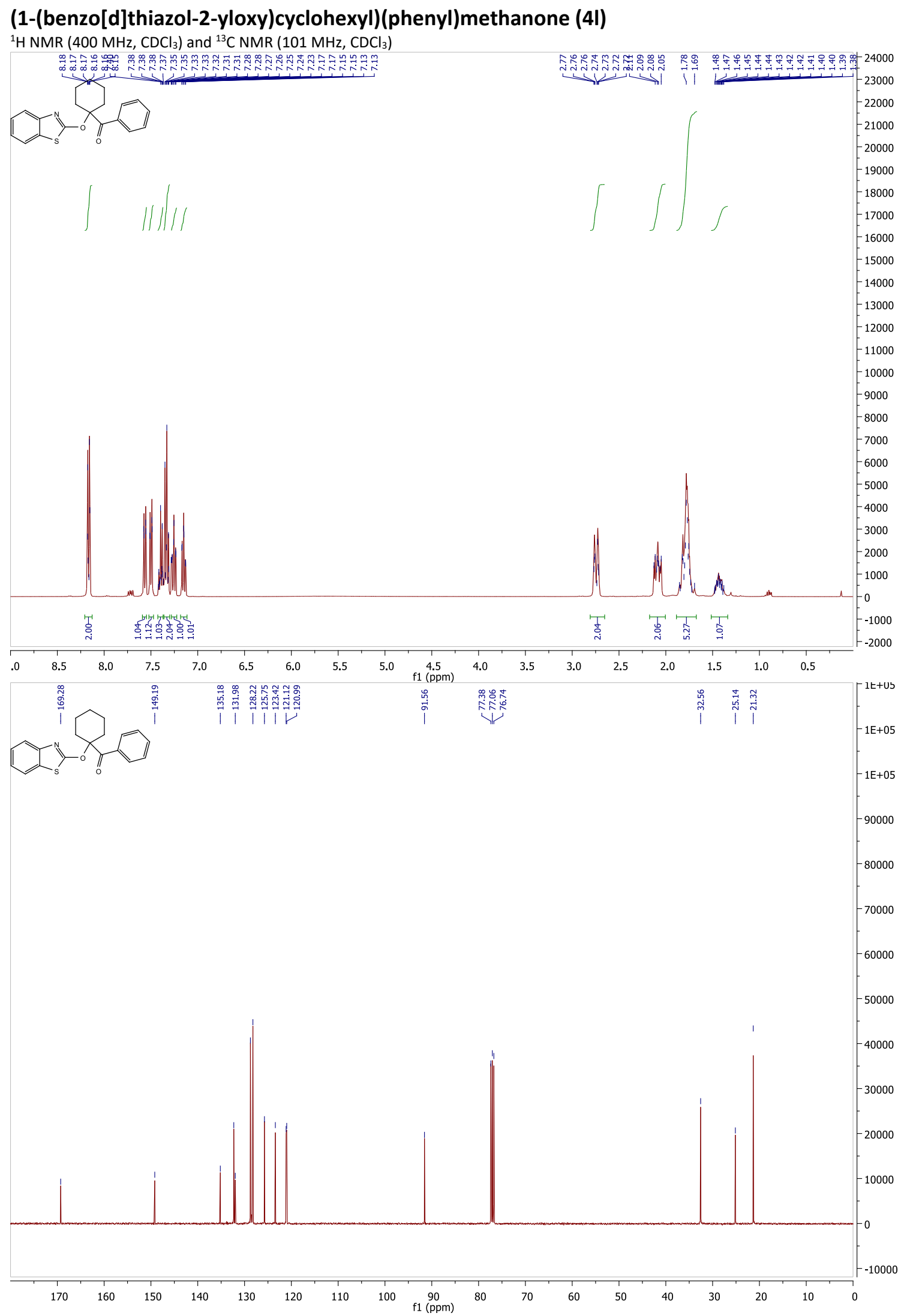


2-(((1R,2S,5R)-2-isopropyl-5-methylcyclohexyl)oxy)benzo[d]thiazole (4m)

${ }^{1} \mathrm{H} \mathrm{NMR}\left(300 \mathrm{MHz}, \mathrm{CDCl}_{3}\right)$ and ${ }^{13} \mathrm{C} \mathrm{NMR}\left(75 \mathrm{MHz}, \mathrm{CDCl}_{3}\right)$

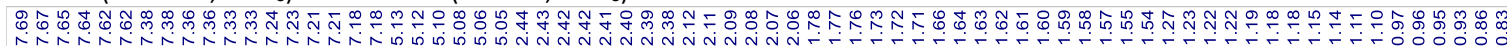
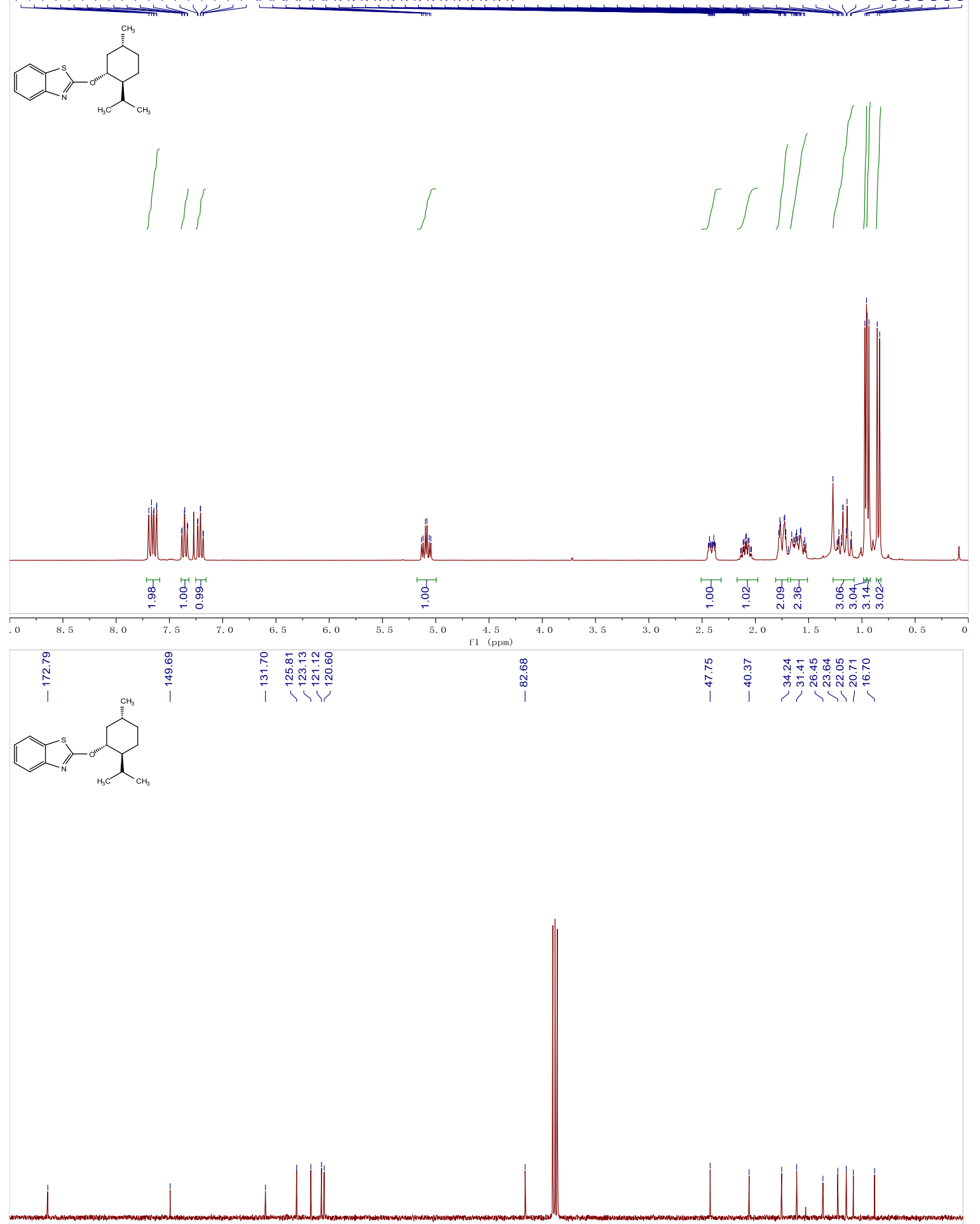

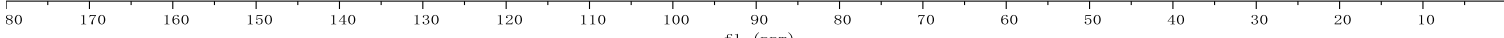


2-(((3S,8R,9S,10R,13R,17R)-13-methyl-17-((R)-6-methylheptan-2-yl)-2,3,4,7,8,9,10,11,12,13,14,15, 16,17-tetradecahydro-1H-cyclopenta[a]phenanthren-3-yl)oxy)benzo[d]thiazole (4n)

${ }^{1} \mathrm{H} \mathrm{NMR}\left(300 \mathrm{MHz}, \mathrm{CDCl}_{3}\right)$ and ${ }^{13} \mathrm{C} \mathrm{NMR}\left(75 \mathrm{MHz}, \mathrm{CDCl}_{3}\right)$

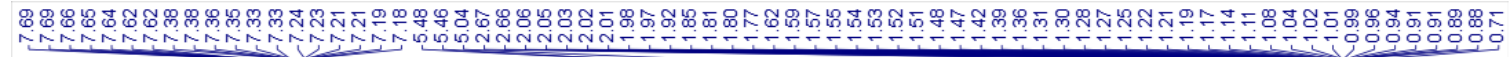
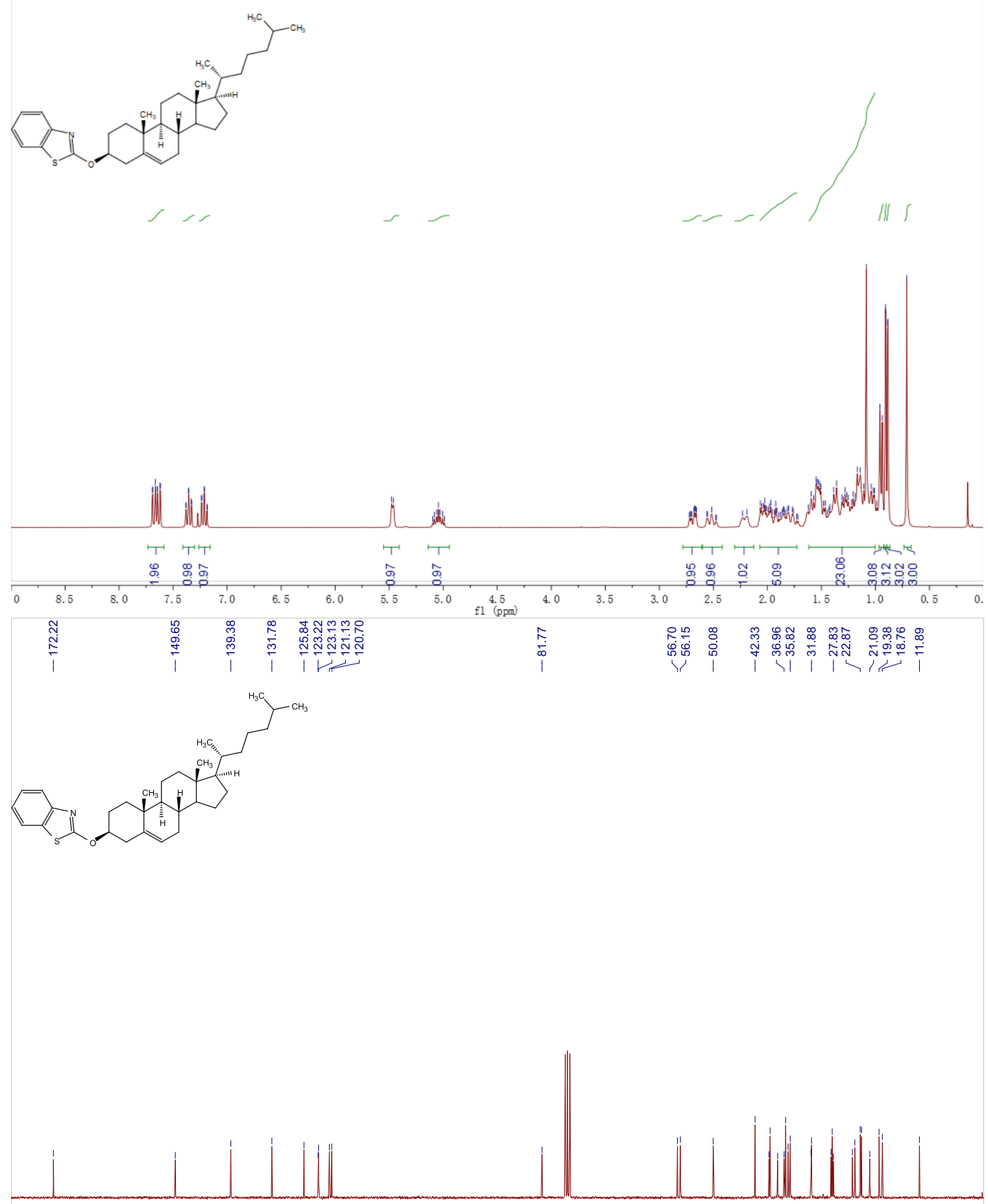

80

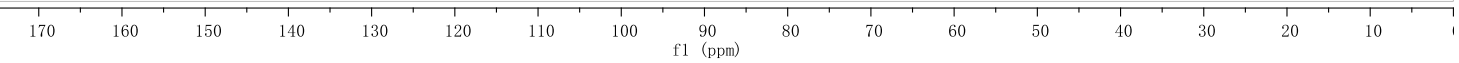




\section{2-phenoxybenzo[d]thiazole (4o)}

${ }^{1} \mathrm{H} \mathrm{NMR}\left(300 \mathrm{MHz}, \mathrm{CDCl}_{3}\right)$ and ${ }^{13} \mathrm{C} \mathrm{NMR}\left(75 \mathrm{MHz}, \mathrm{CDCl}_{3}\right)$

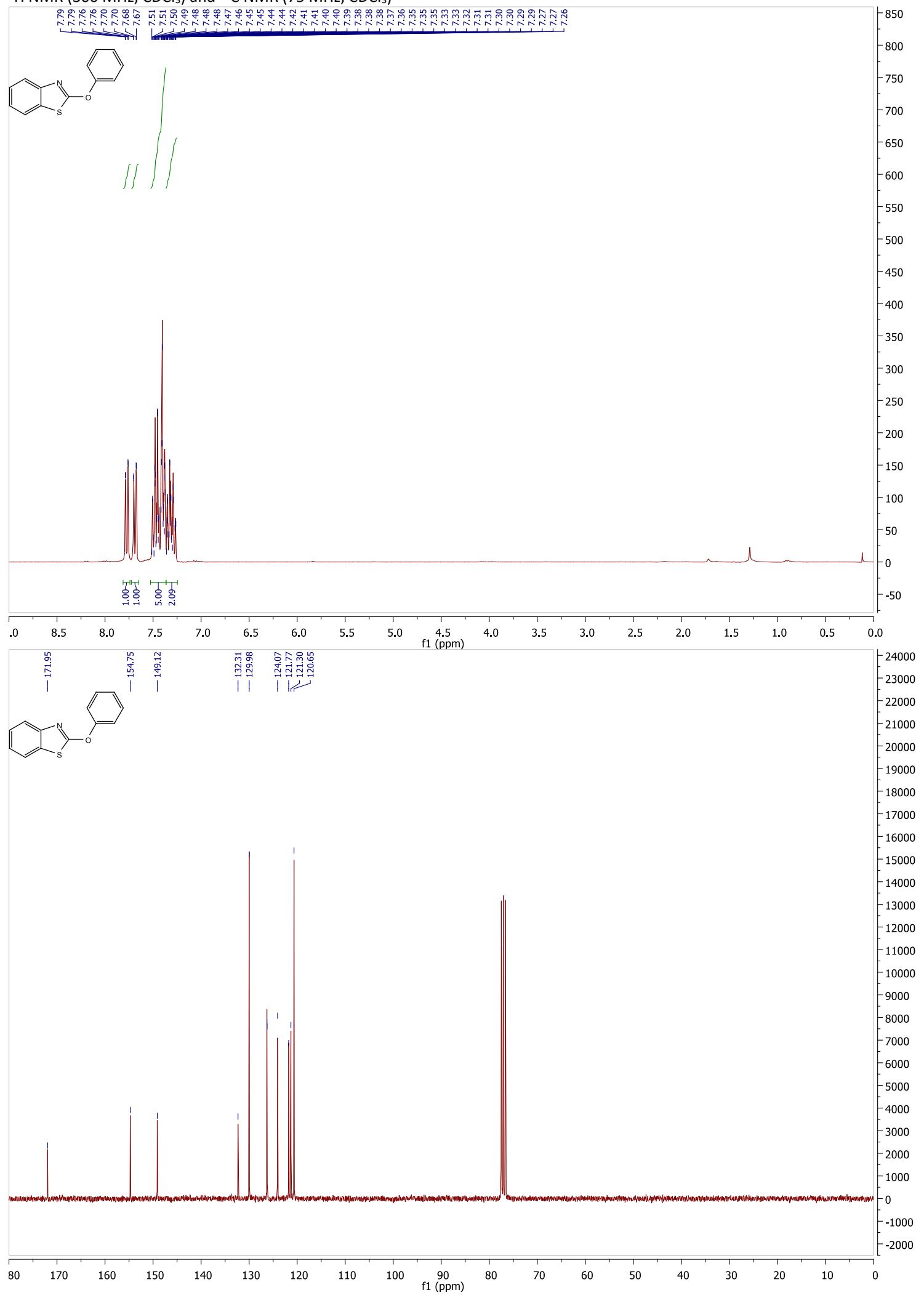




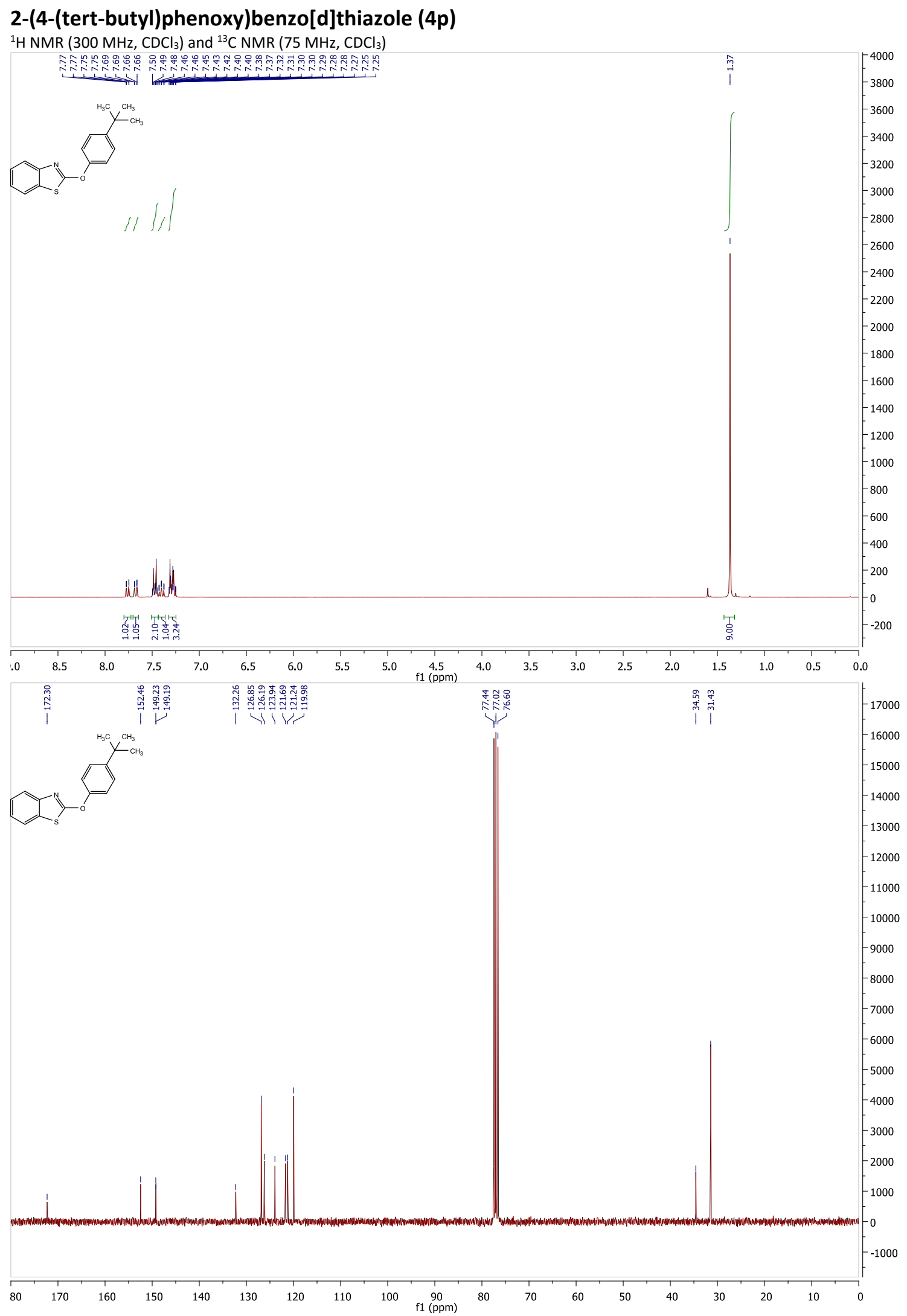




\section{2-(4-methoxyphenoxy)benzo[d]thiazole (4q)}

${ }^{1} \mathrm{H} \mathrm{NMR}\left(300 \mathrm{MHz}, \mathrm{CDCl}_{3}\right)$ and ${ }^{13} \mathrm{C} \mathrm{NMR}\left(75 \mathrm{MHz}, \mathrm{CDCl}_{3}\right)$

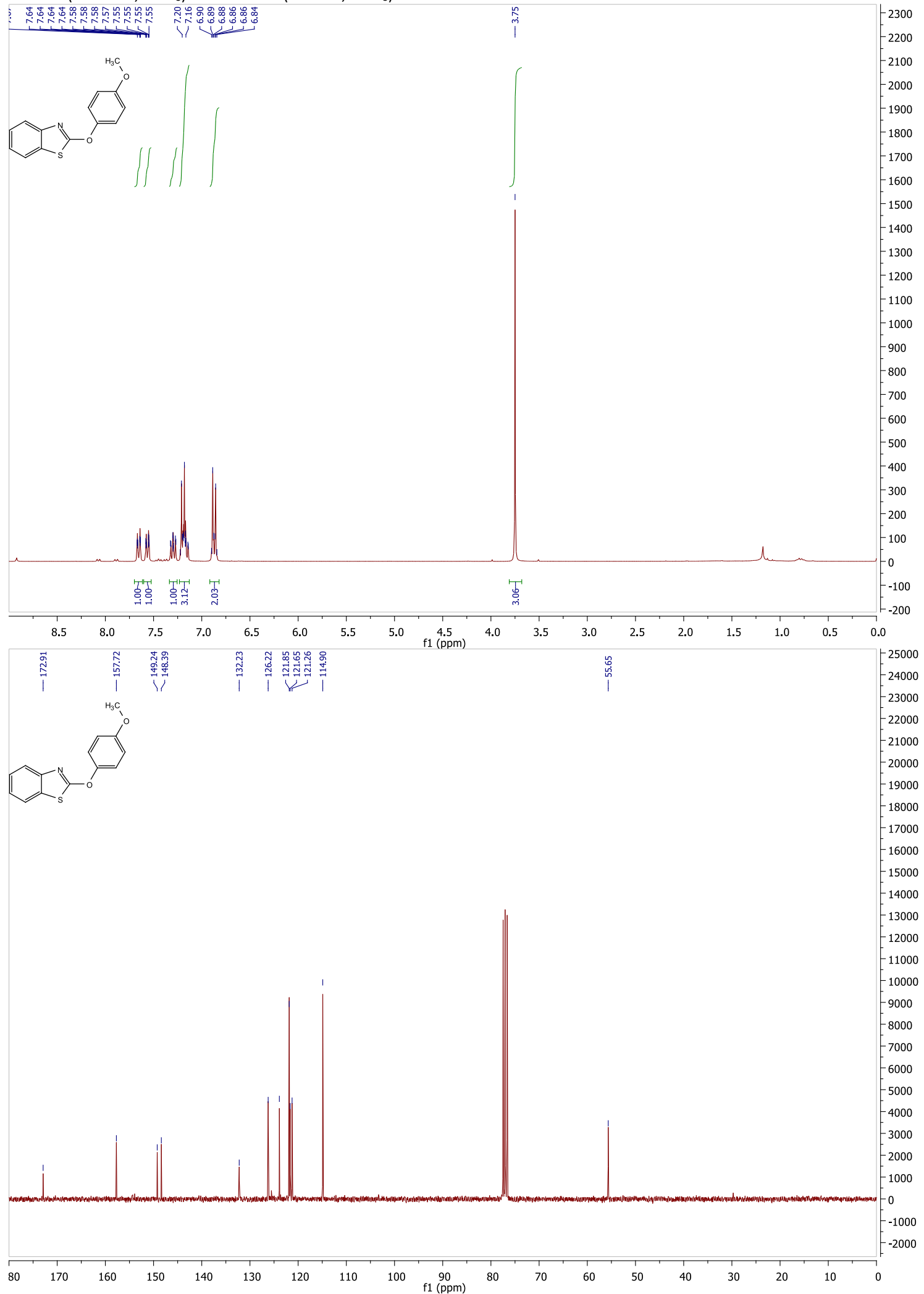


2-(p-tolyloxy)benzo[d]thiazole (4r)

${ }^{1} \mathrm{H} \mathrm{NMR}\left(300 \mathrm{MHz}, \mathrm{CDCl}_{3}\right)$ and ${ }^{13} \mathrm{C} \mathrm{NMR}\left(75 \mathrm{MHz}, \mathrm{CDCl}_{3}\right)$

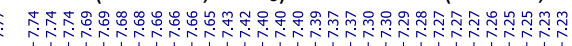

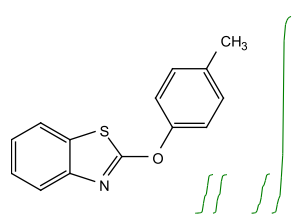

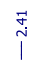

4000

3500

3000
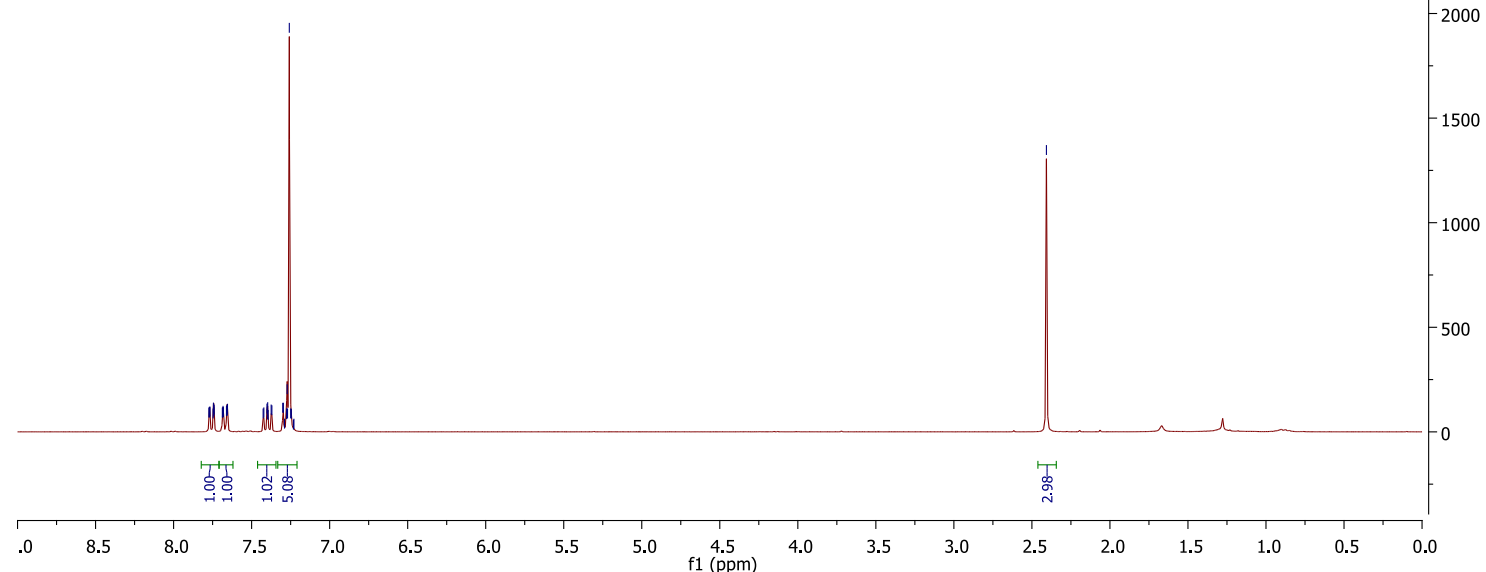

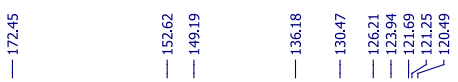
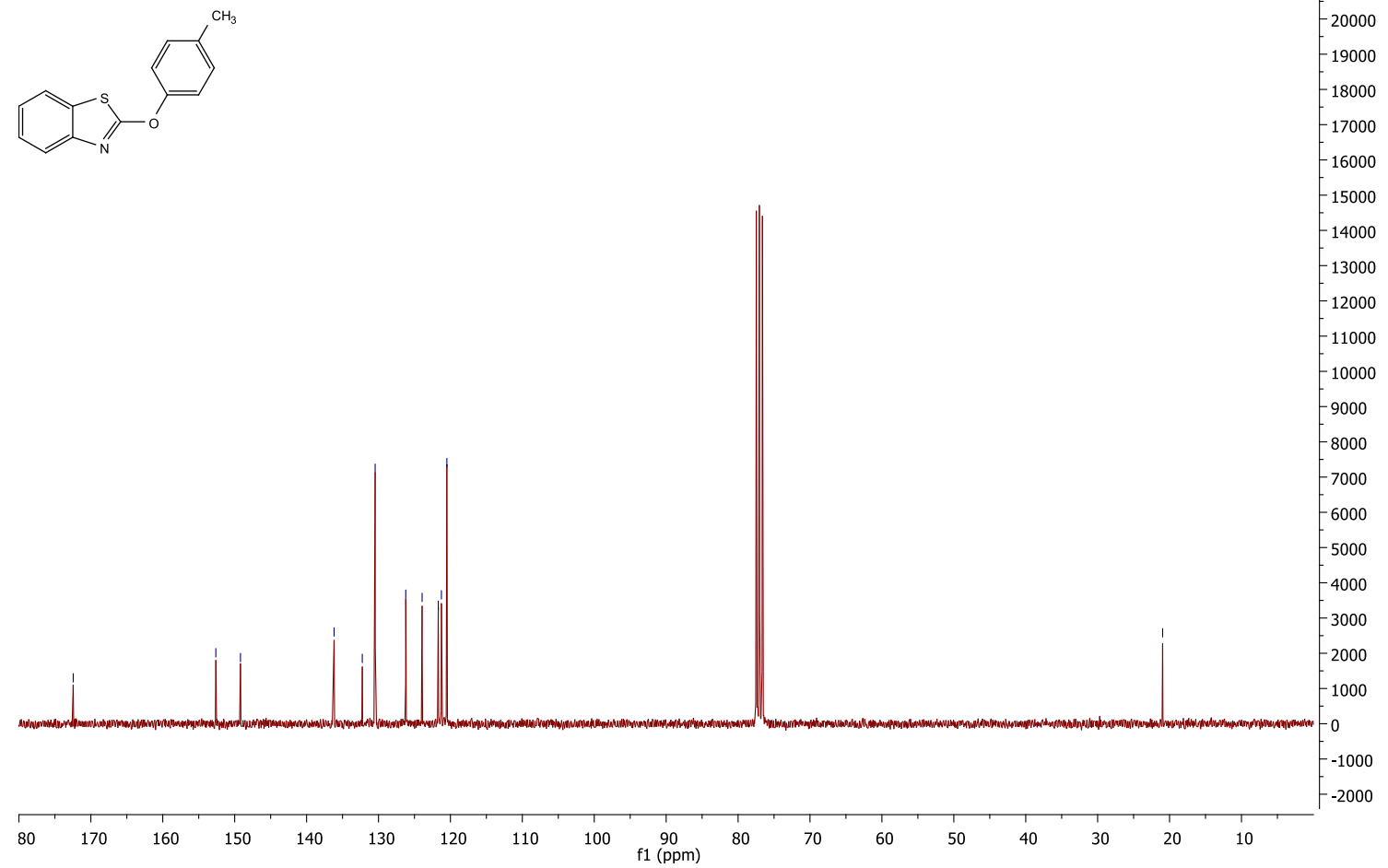


\section{2-(m-tolyloxy)benzo[d]thiazole (4s)}

${ }^{1} \mathrm{H} \mathrm{NMR}\left(400 \mathrm{MHz}, \mathrm{CDCl}_{3}\right)$ and ${ }^{13} \mathrm{C} \mathrm{NMR} \mathrm{(101} \mathrm{MHz,} \mathrm{CDCl} 3$ )

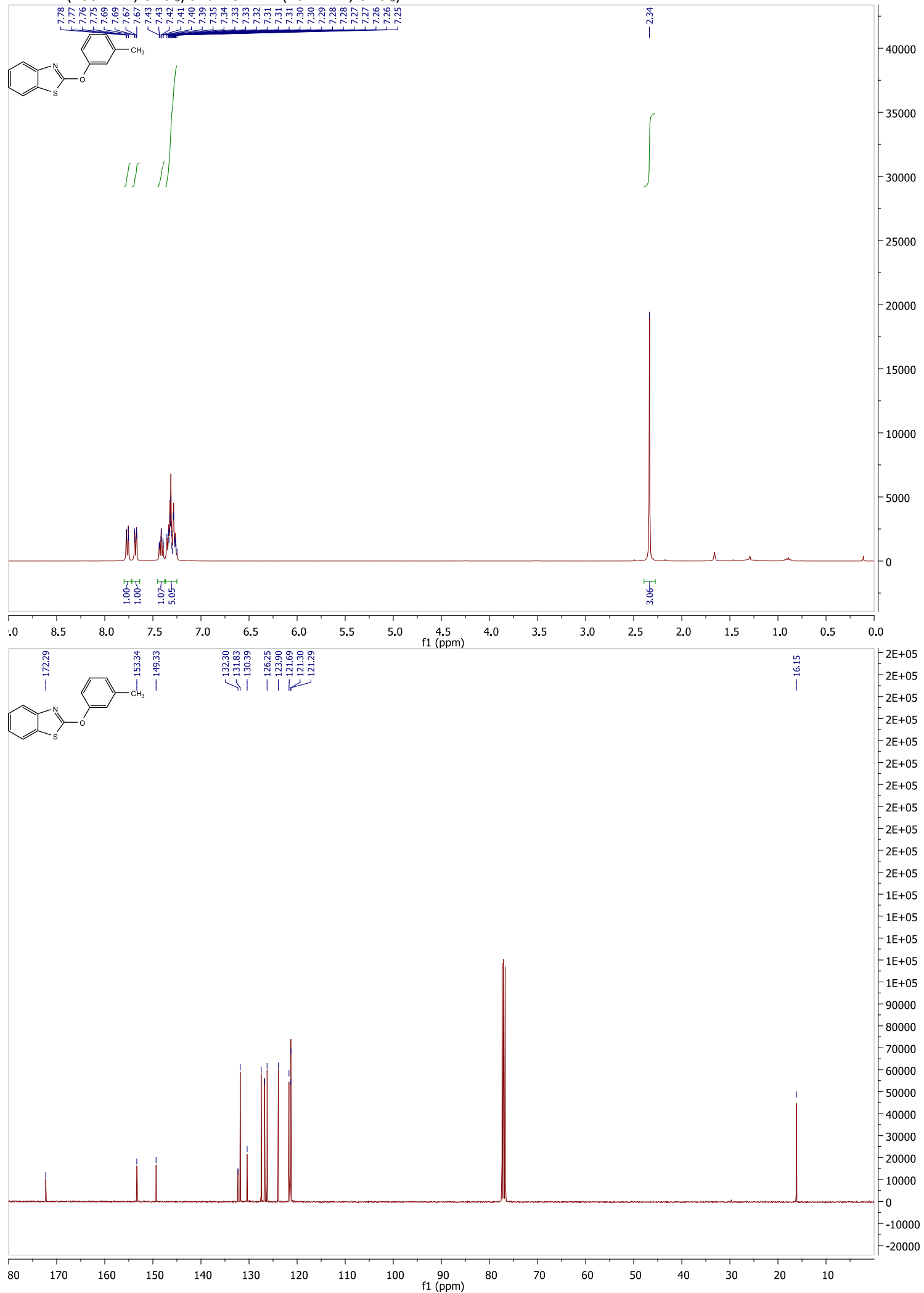


2-(o-tolyloxy)benzo[d]thiazole (4t)

${ }^{1} \mathrm{H} \mathrm{NMR}\left(300 \mathrm{MHz}, \mathrm{CDCl}_{3}\right)$ and ${ }^{13} \mathrm{C} \mathrm{NMR}\left(75 \mathrm{MHz}, \mathrm{CDCl}_{3}\right)$

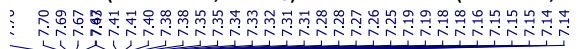<smiles>C=CCOc1c(C)cccc1COc1nc2ccccc2s1</smiles>
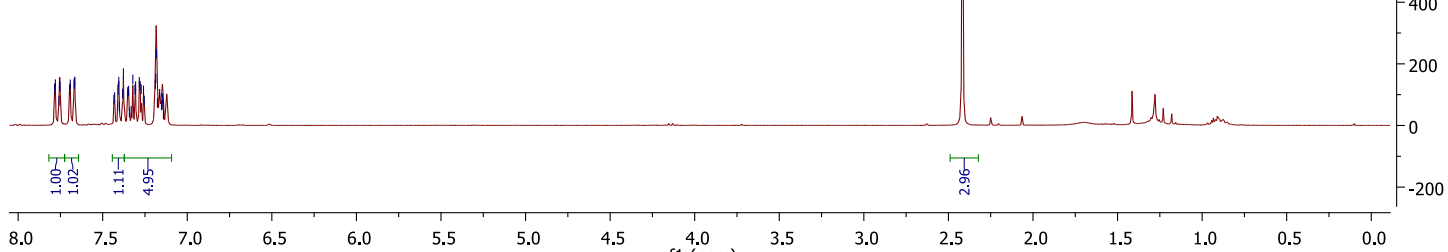

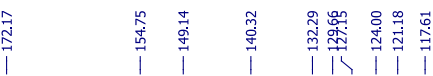<smiles>Cc1ccccc1Oc1nc2ccccc2s1</smiles>

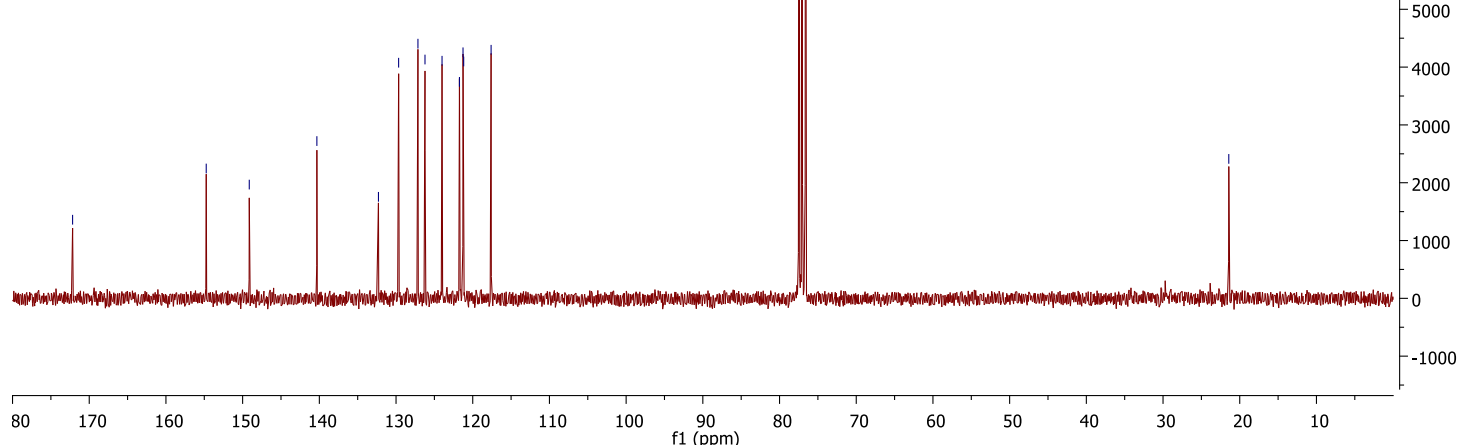




\section{1,4-bis(benzo[d]thiazol-2-yloxy)benzene (4u)}

${ }^{1} \mathrm{H}$ NMR $\left(600 \mathrm{MHz}\right.$, DMSO- $\left.d_{6}\right)$ and ${ }^{13} \mathrm{C}$ NMR $\left(151 \mathrm{MHz}\right.$, DMSO- $\left.d_{6}\right)$

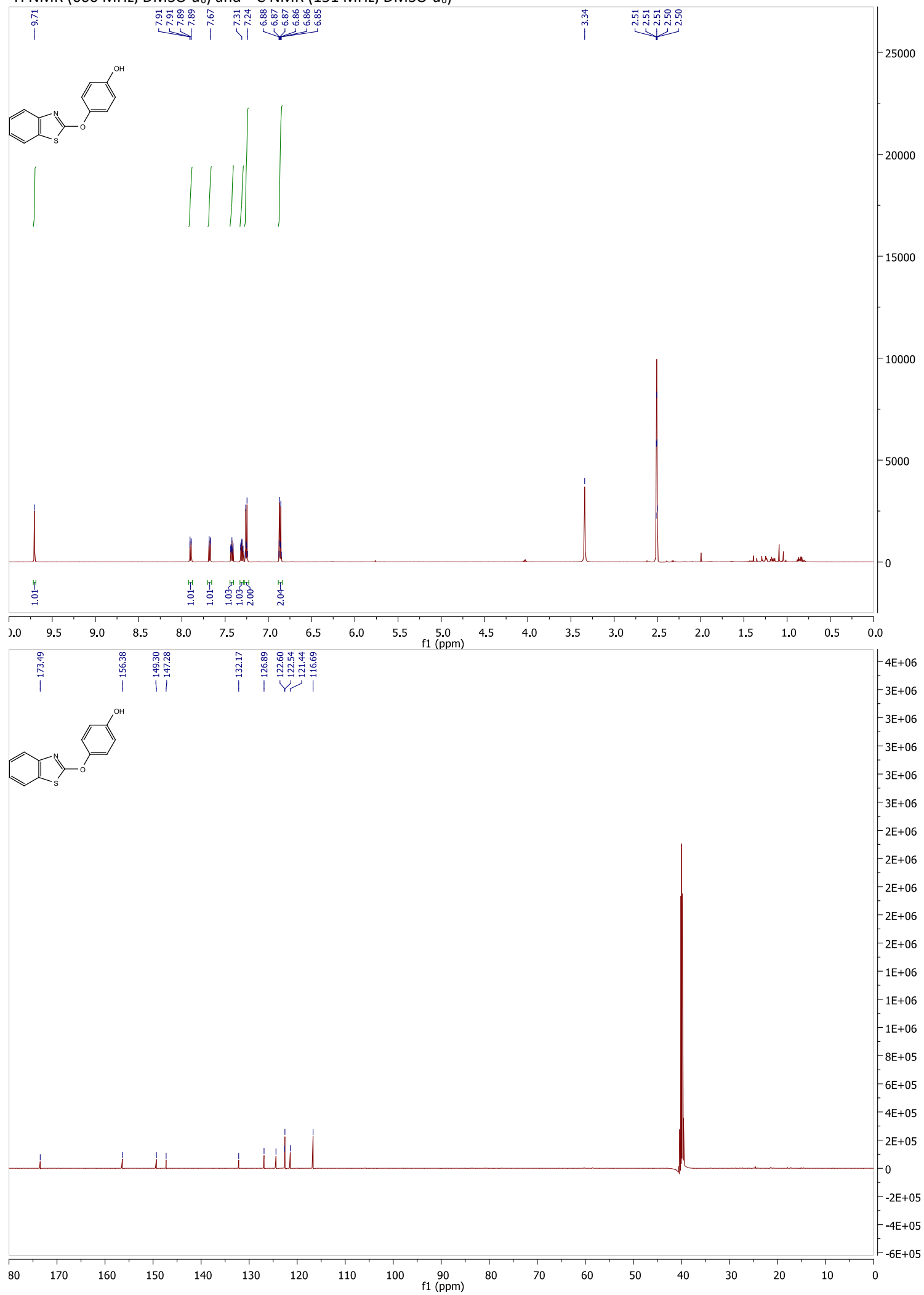




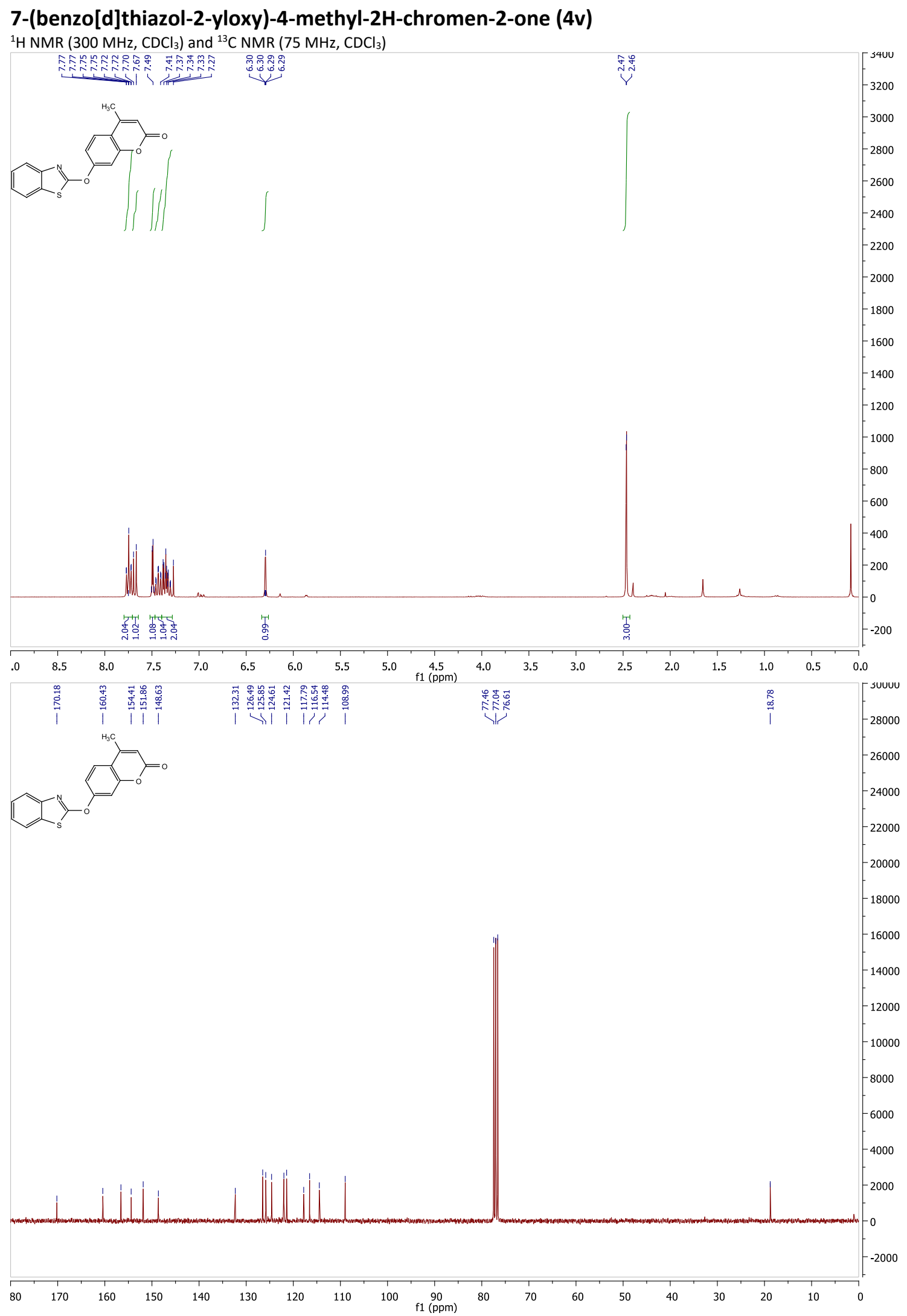




\section{2-(naphthalen-1-yloxy)benzo[d]thiazole (4w)}

${ }^{1} \mathrm{H} \mathrm{NMR}\left(300 \mathrm{MHz}, \mathrm{CDCl}_{3}\right)$ and ${ }^{13} \mathrm{C} \mathrm{NMR}\left(75 \mathrm{MHz}, \mathrm{CDCl}_{3}\right)$

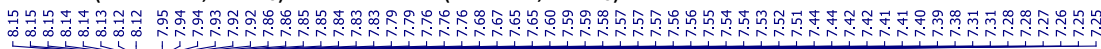<smiles>c1ccc2c(Oc3nc4ccccc4s3)cccc2c1</smiles>
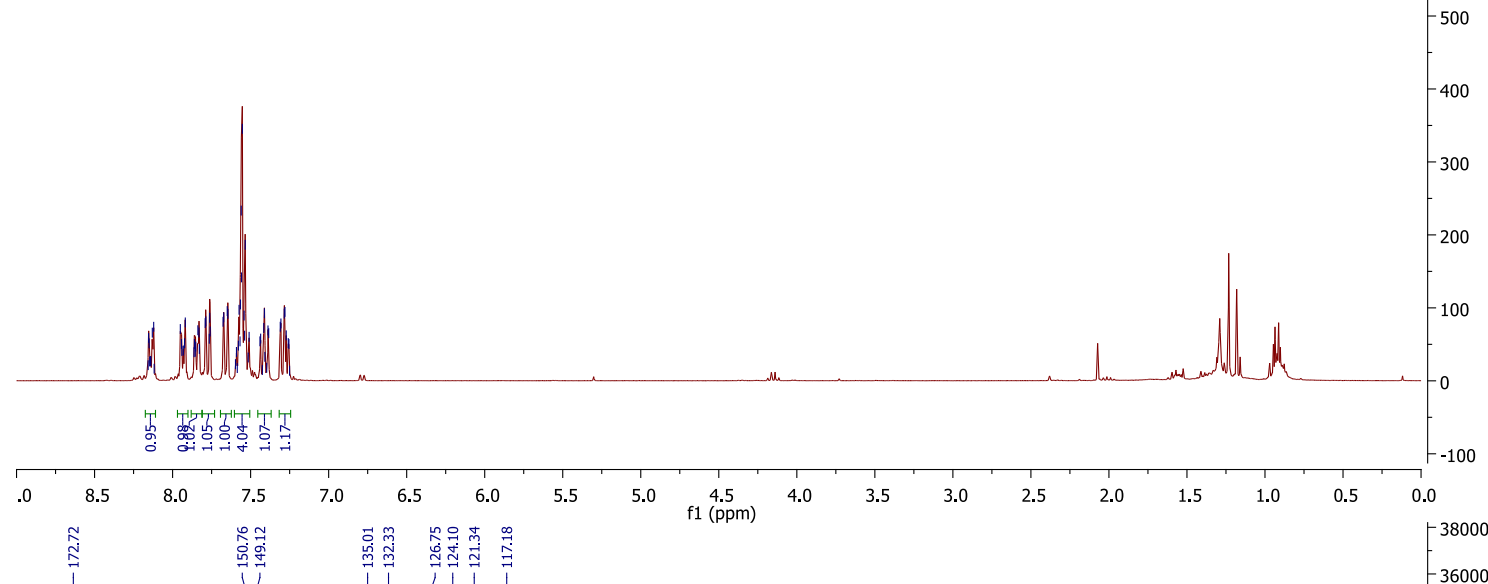

$\vec{l} \quad \vec{l} \quad \vec{l}$ i
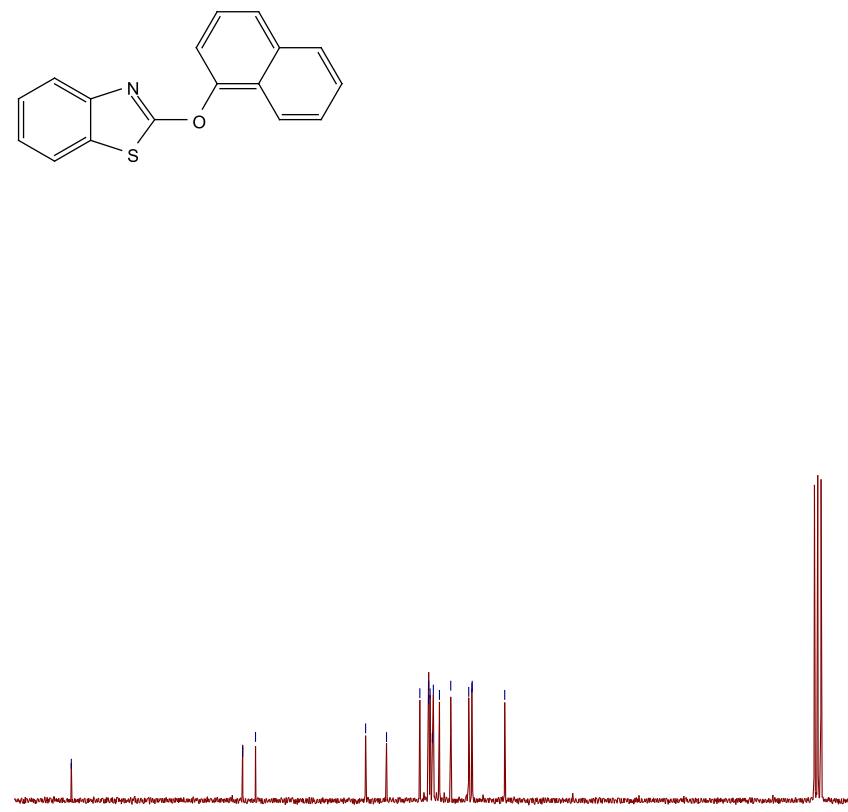

16000

$-14000$

12000

10000

8000

6000

4000

$-2000$ 


\section{2-(4-bromophenoxy)benzo[d]thiazole (4x)}

${ }^{1} \mathrm{H} \mathrm{NMR}\left(300 \mathrm{MHz}, \mathrm{CDCl}_{3}\right)$ and ${ }^{13} \mathrm{C} \mathrm{NMR}\left(75 \mathrm{MHz}, \mathrm{CDCl}_{3}\right)$

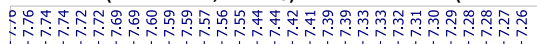

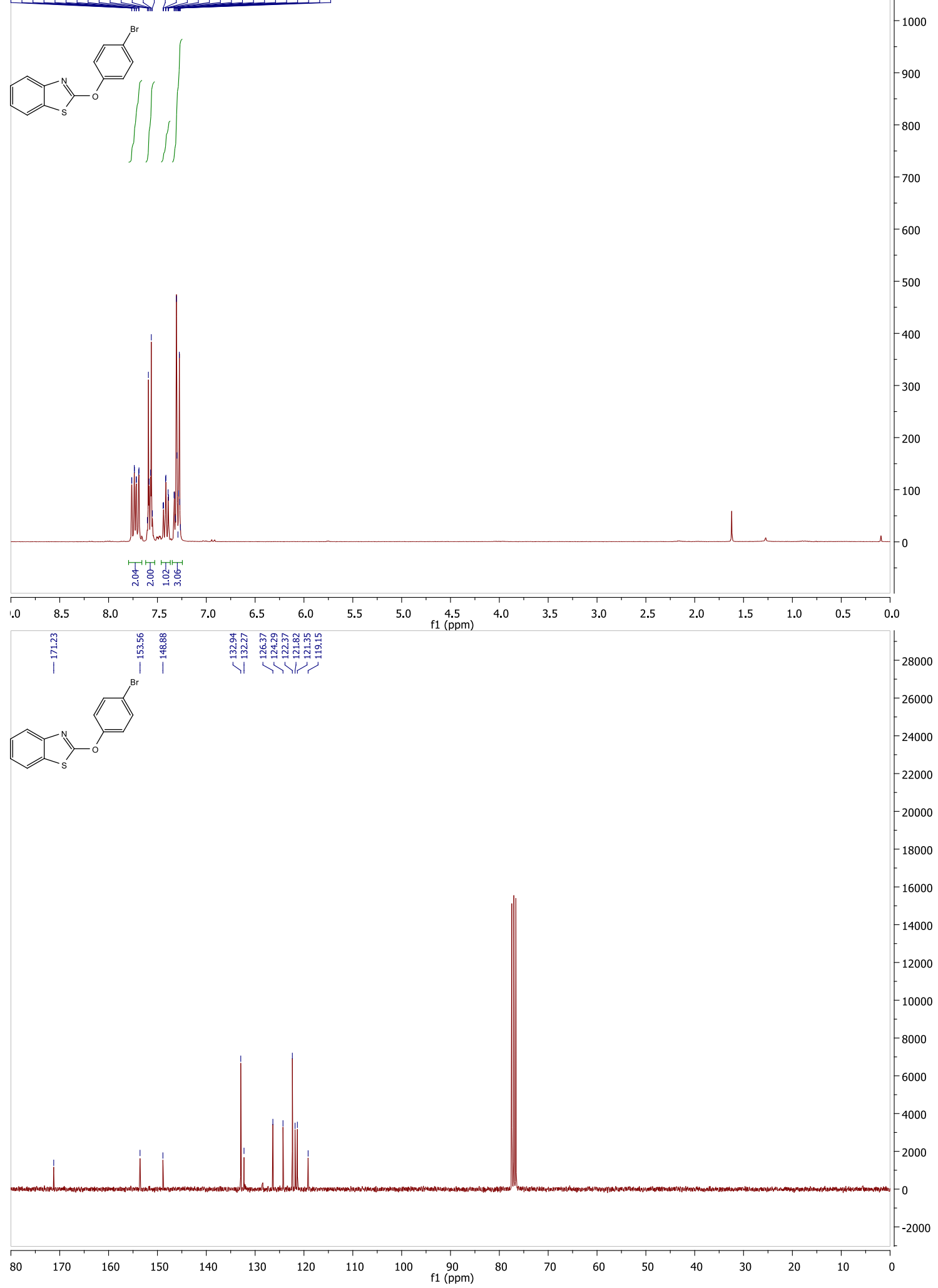


2-(3-bromophenoxy)benzo[d]thiazole (4y)

${ }^{1} \mathrm{H} \mathrm{NMR}\left(300 \mathrm{MHz}, \mathrm{CDCl}_{3}\right)$ and ${ }^{13} \mathrm{C} \mathrm{NMR}\left(75 \mathrm{MHz}, \mathrm{CDCl}_{3}\right)$

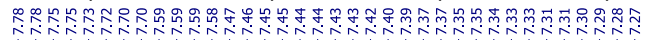
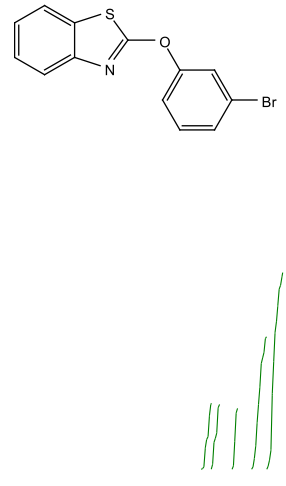

$-700$

$-600$

500

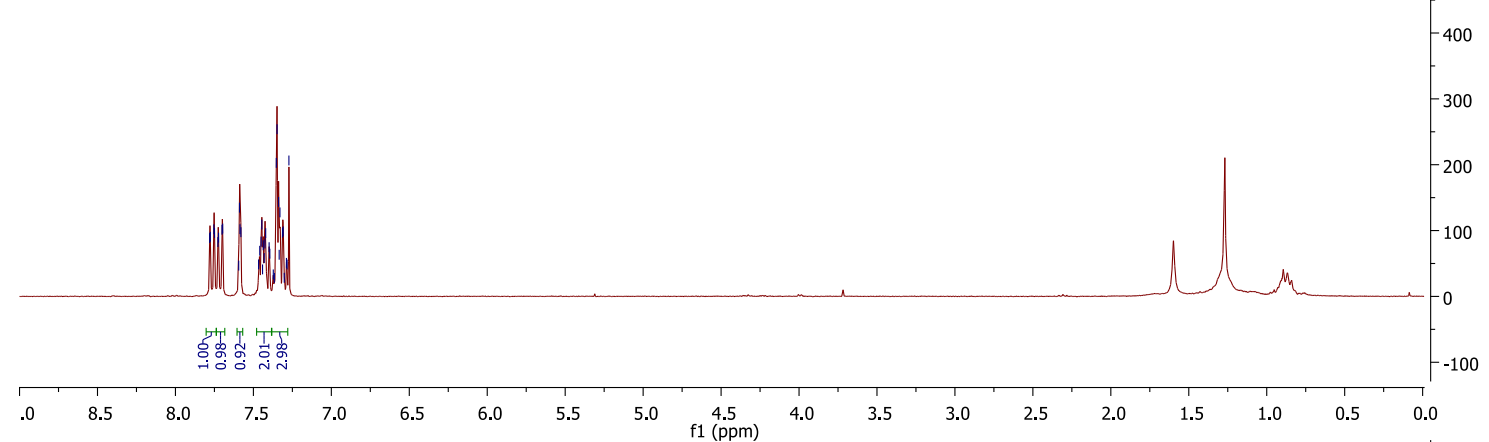

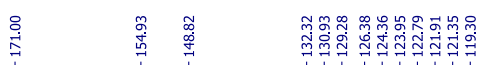

$\vec{i} \mid \vec{i}$

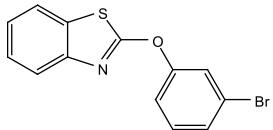

(1)
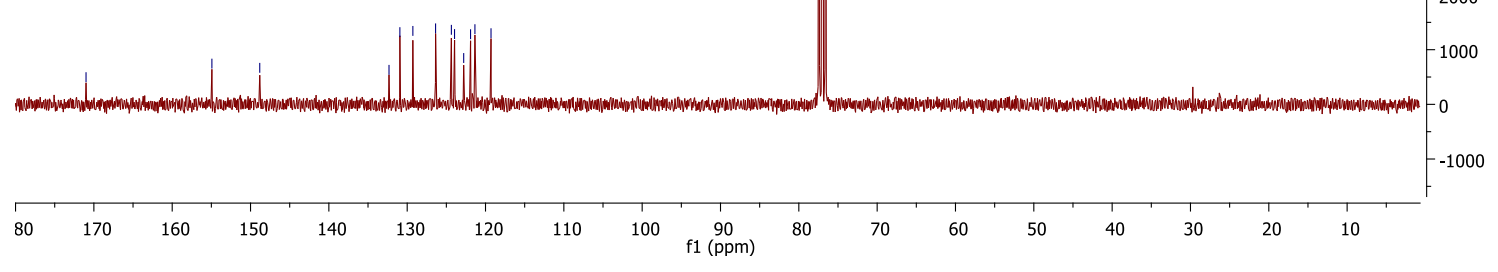
2-(2-bromophenoxy)benzo[d]thiazole (4z)

${ }^{1} \mathrm{H} \mathrm{NMR}\left(300 \mathrm{MHz}, \mathrm{CDCl}_{3}\right)$ and ${ }^{13} \mathrm{C} \mathrm{NMR}\left(75 \mathrm{MHz}, \mathrm{CDCl}_{3}\right)$

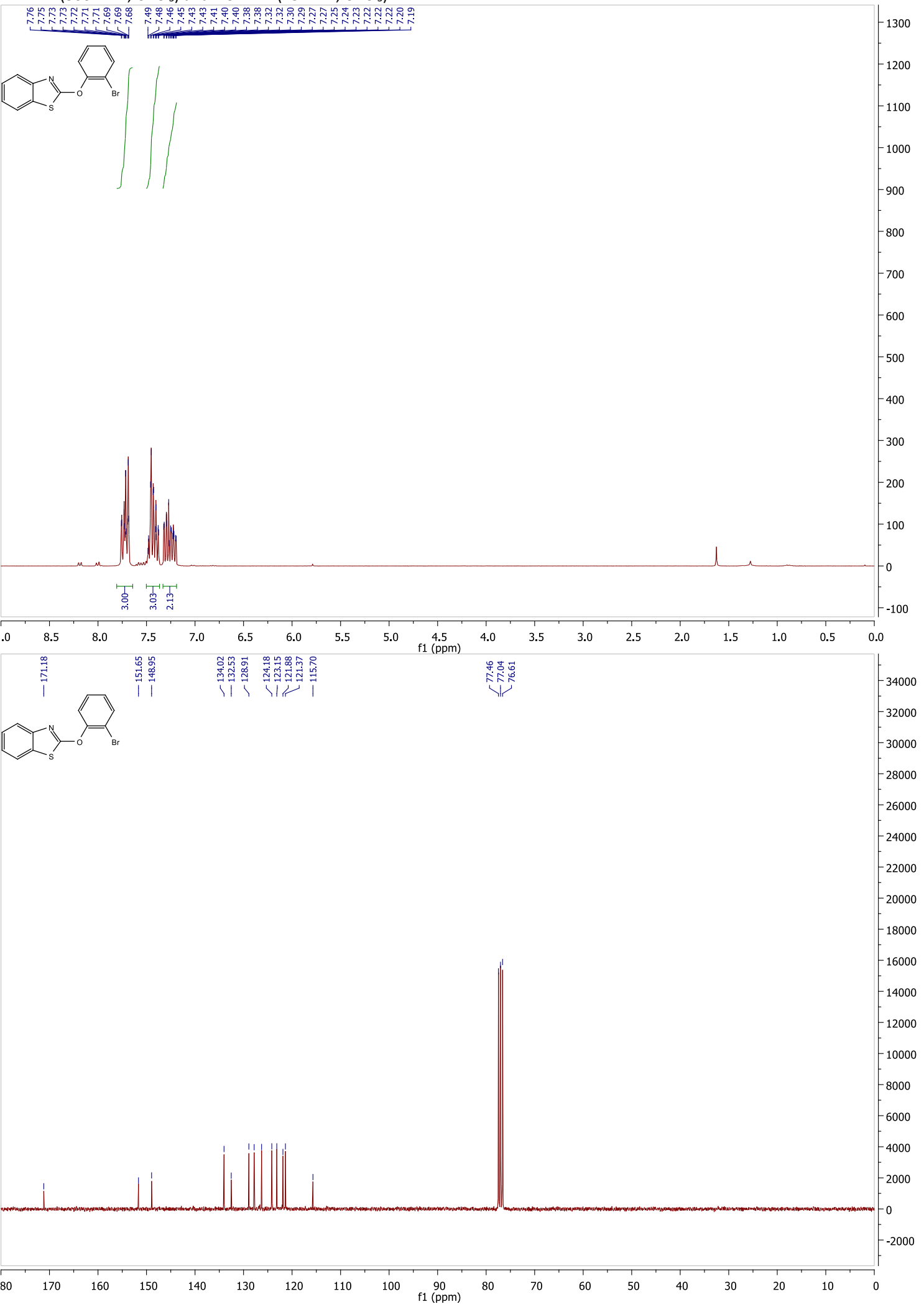


2-(2,4-difluorophenoxy)benzo[d]thiazole (4aa)

$\left.{ }^{1} \mathrm{H} \mathrm{NMR} \mathrm{(300} \mathrm{MHz,} \mathrm{CDCl}_{3}\right)$ and ${ }^{13} \mathrm{C} \mathrm{NMR}\left(75 \mathrm{MHz}, \mathrm{CDCl}_{3}\right)$

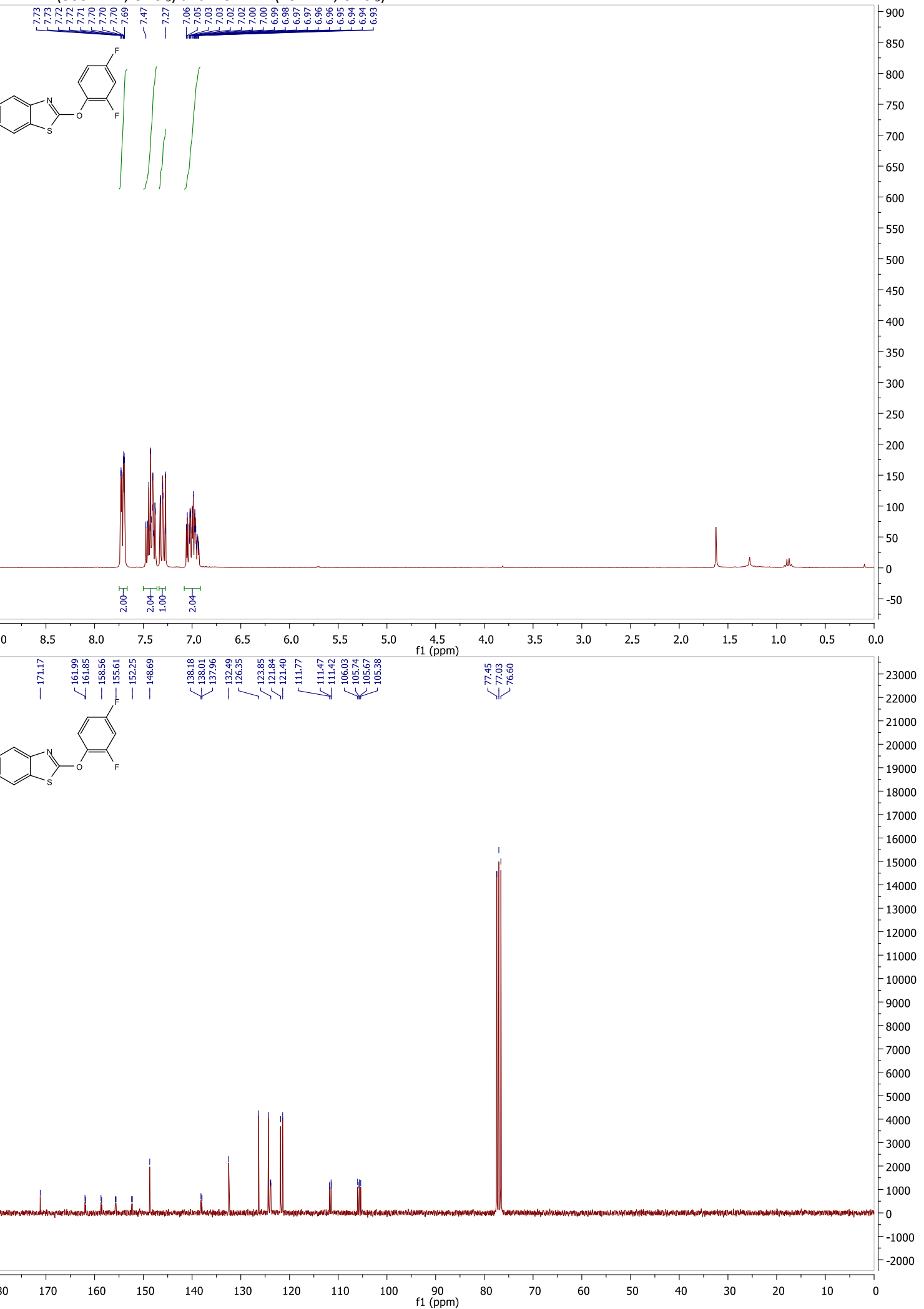




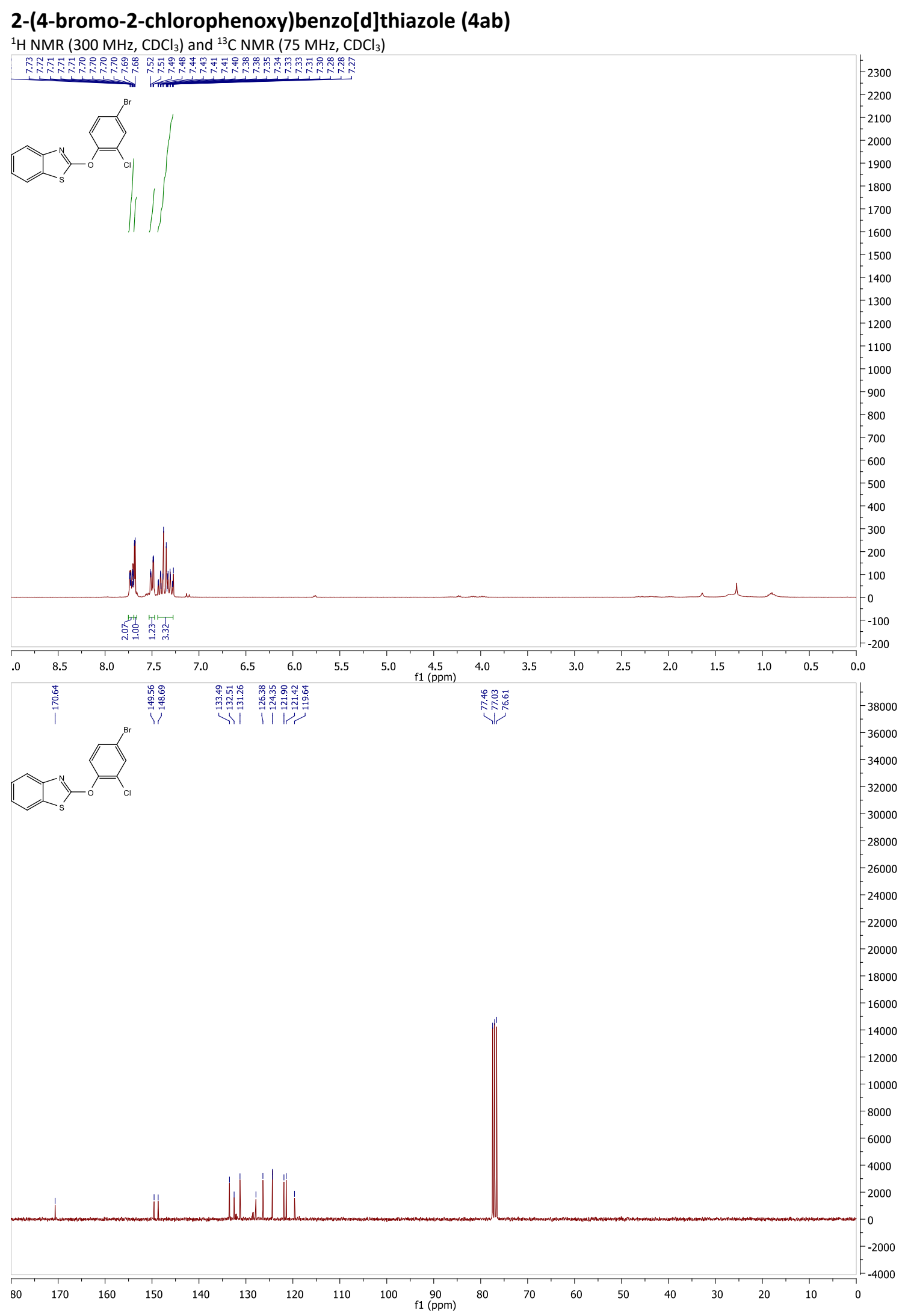




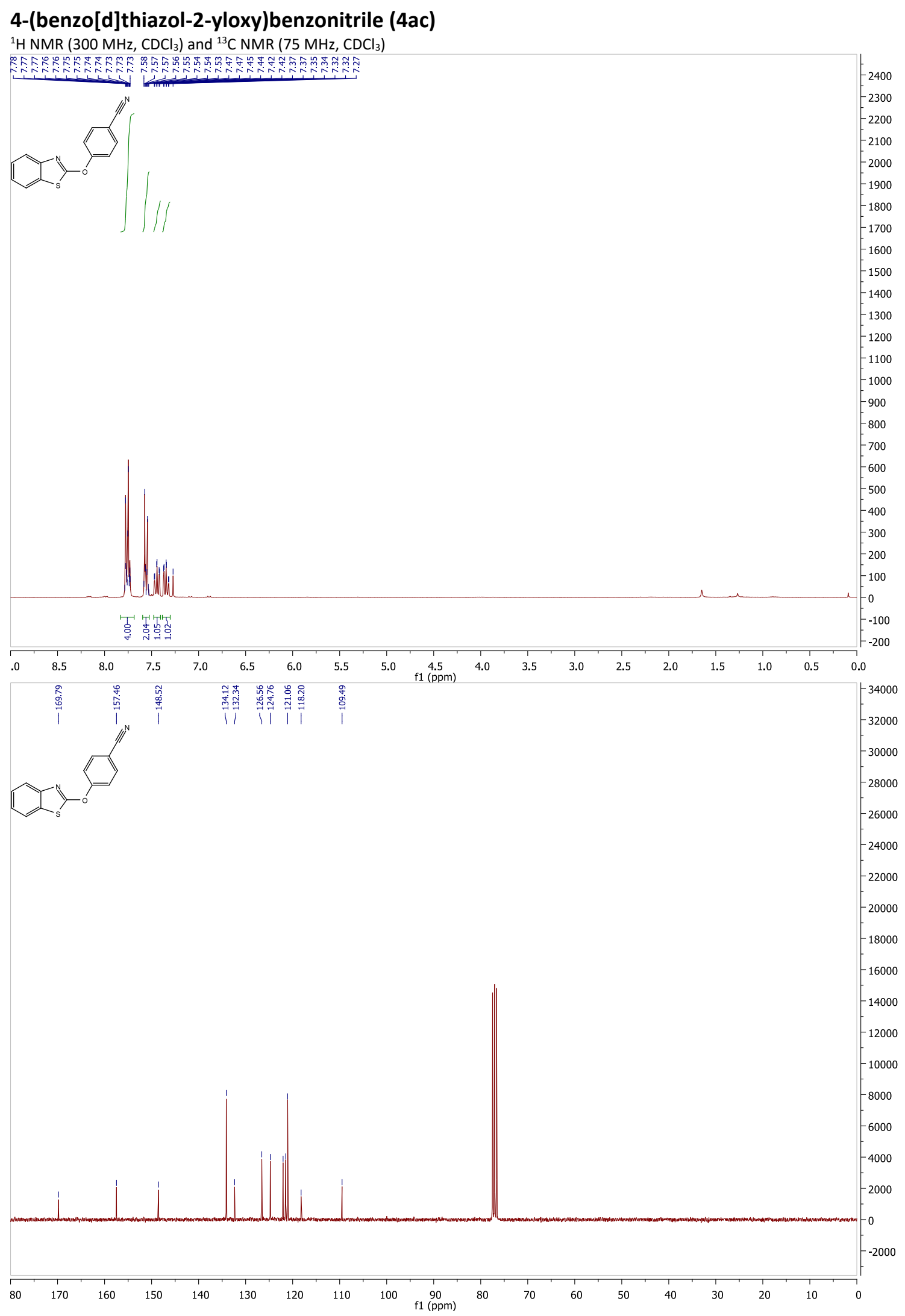




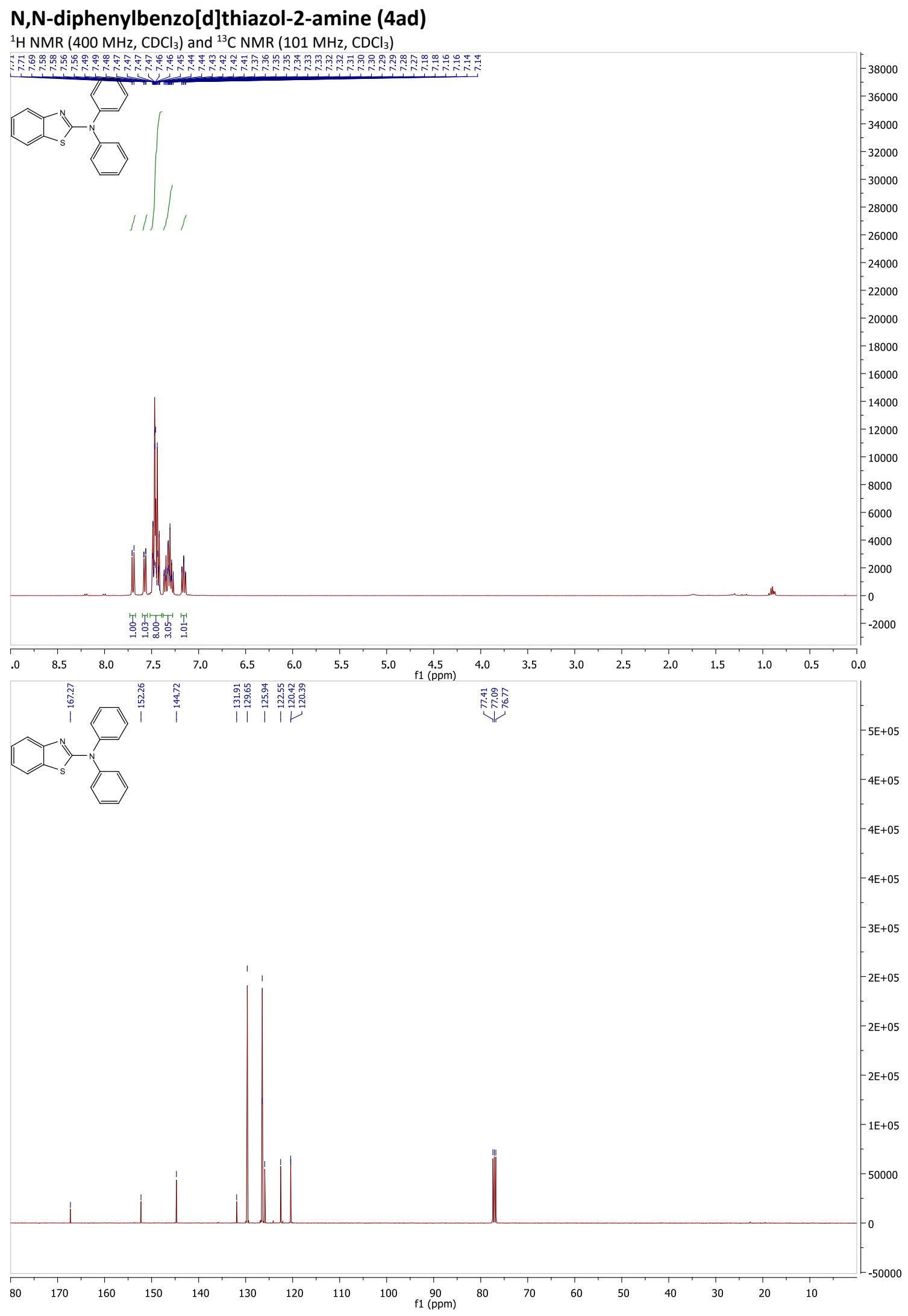




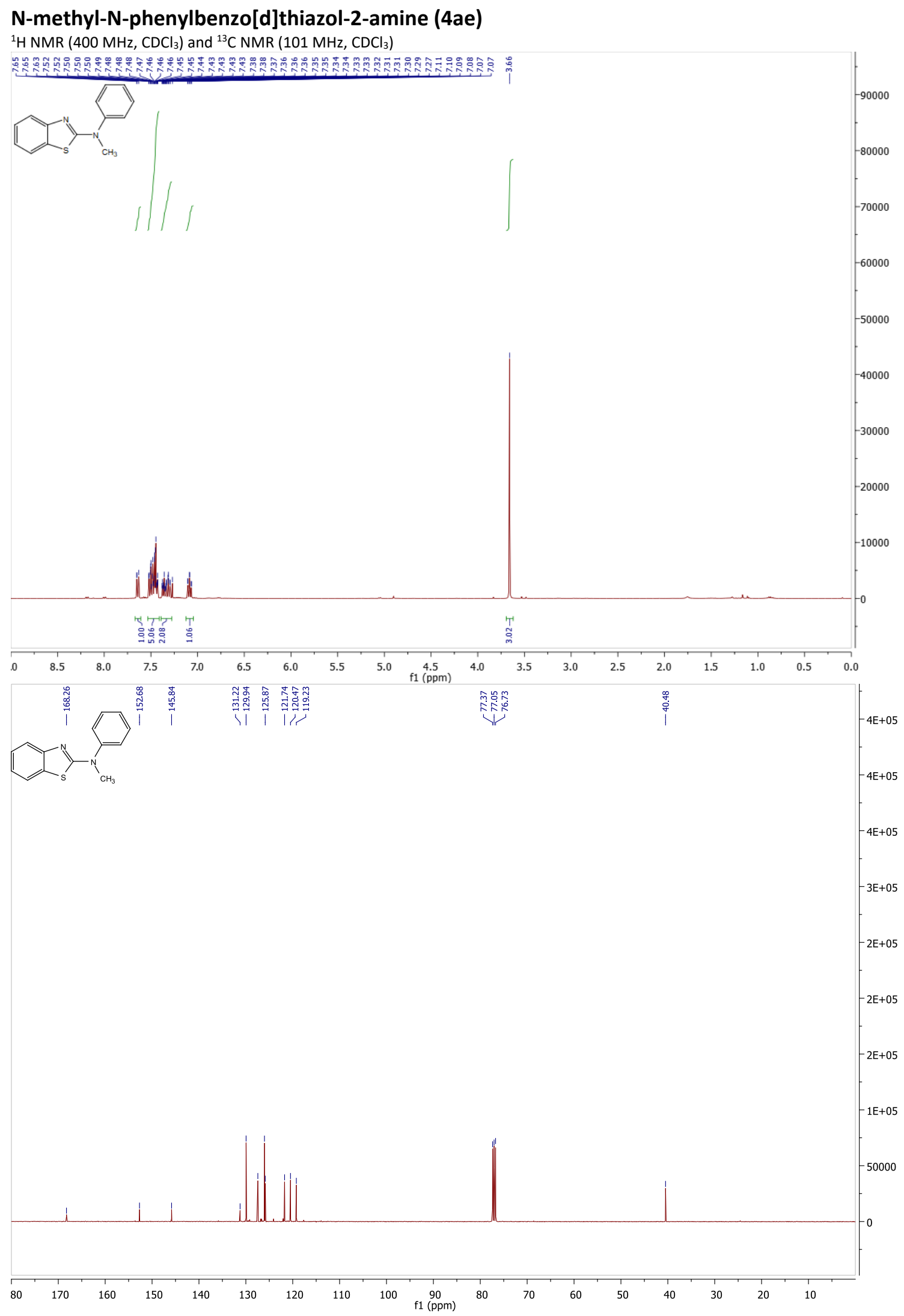




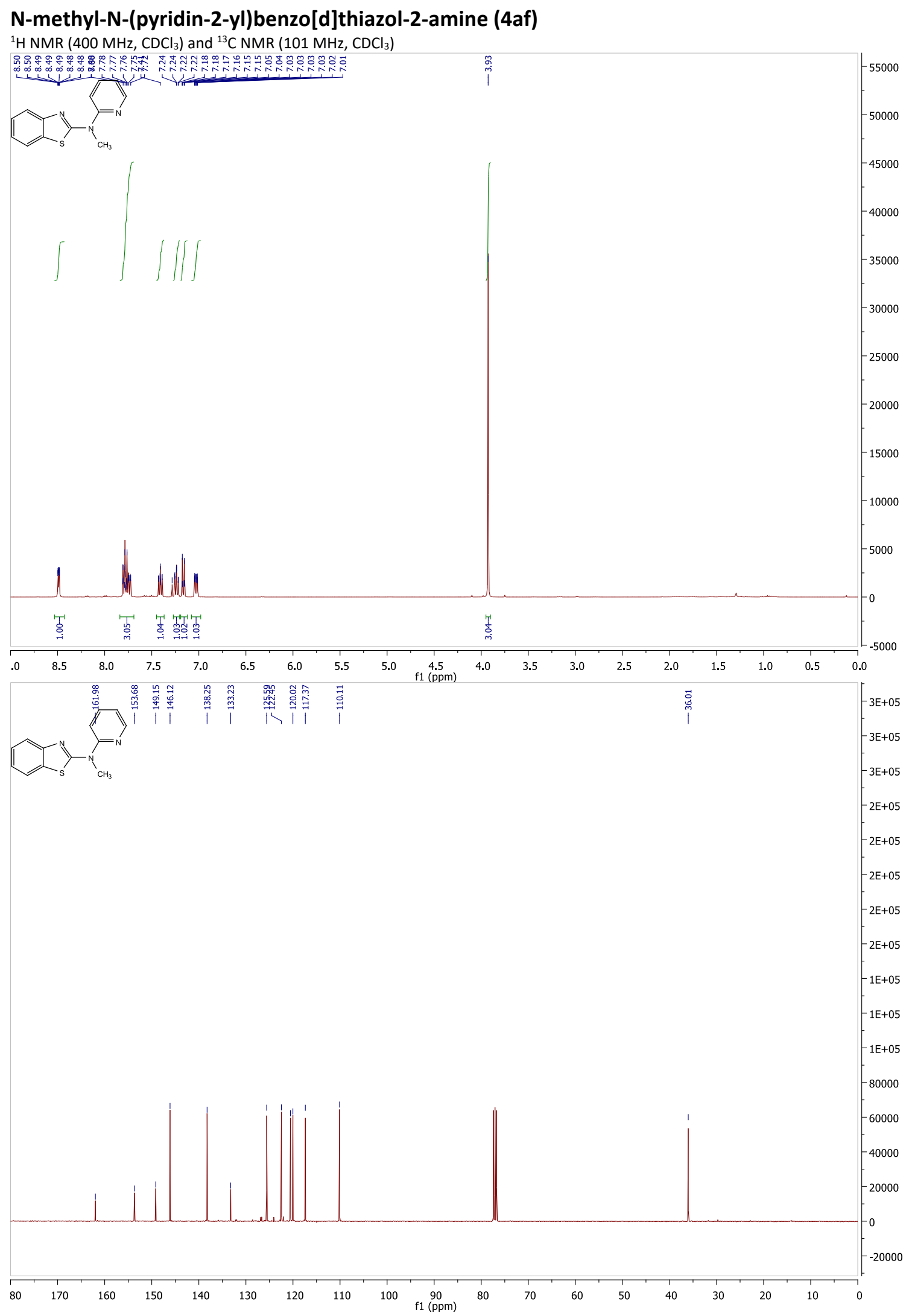




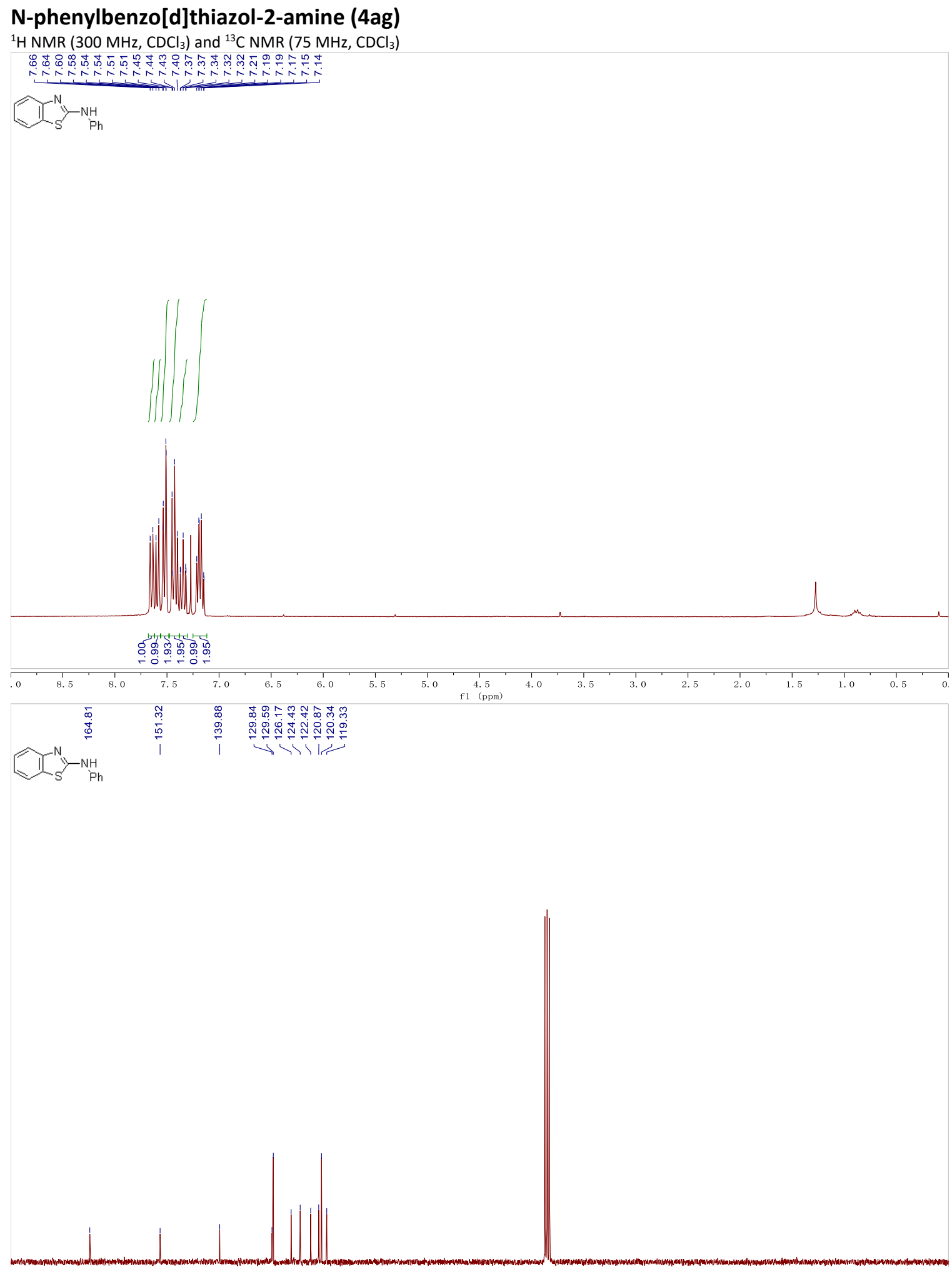

(1)

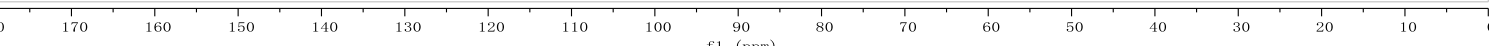


$\mathbf{N}$-(benzo[d]thiazol-2-yl)-N-methylbenzamide (4ah)

${ }^{1} \mathrm{H} \mathrm{NMR}\left(300 \mathrm{MHz}, \mathrm{CDCl}_{3}\right)$ and ${ }^{13} \mathrm{C} \mathrm{NMR}\left(75 \mathrm{MHz}, \mathrm{CDCl}_{3}\right)$

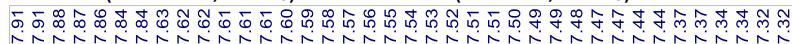
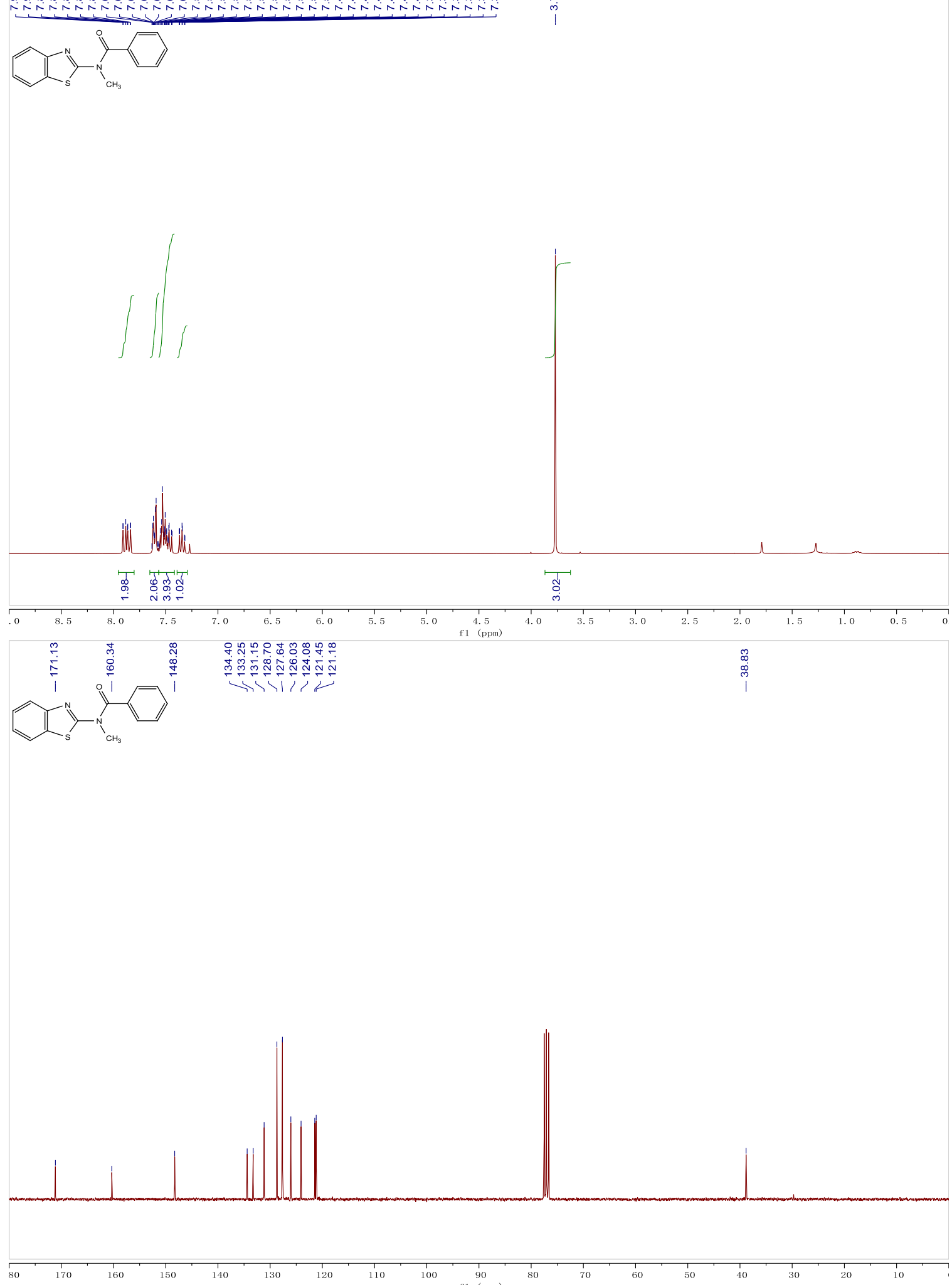

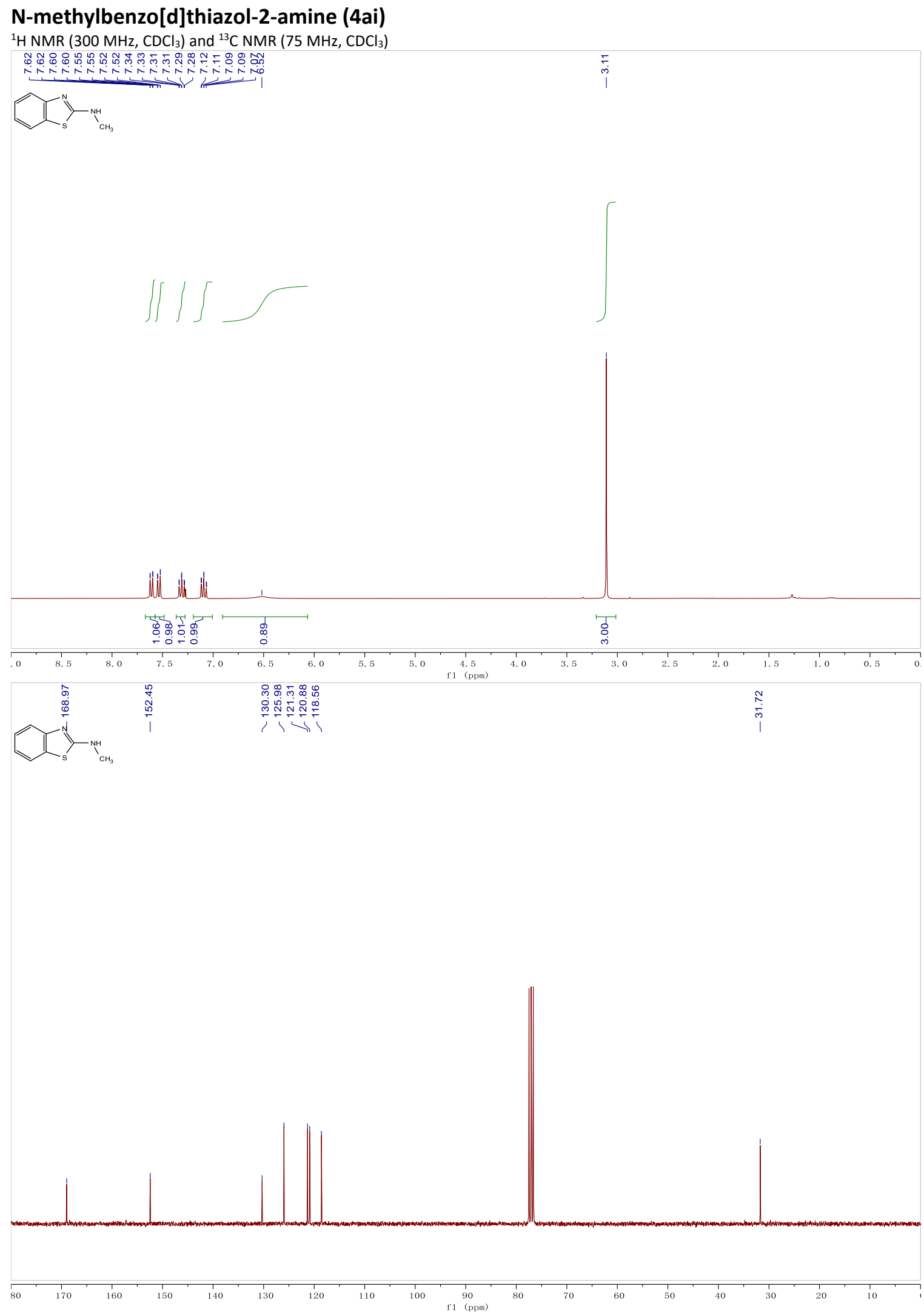
2-(1H-pyrrol-1-yl)benzo[d]thiazole (4aj)

$\left.{ }^{1} \mathrm{H} \mathrm{NMR} \mathrm{(300} \mathrm{MHz}, \mathrm{CDCl}_{3}\right)$ and ${ }^{13} \mathrm{C} \mathrm{NMR}\left(75 \mathrm{MHz}, \mathrm{CDCl}_{3}\right)$ 我

(N)

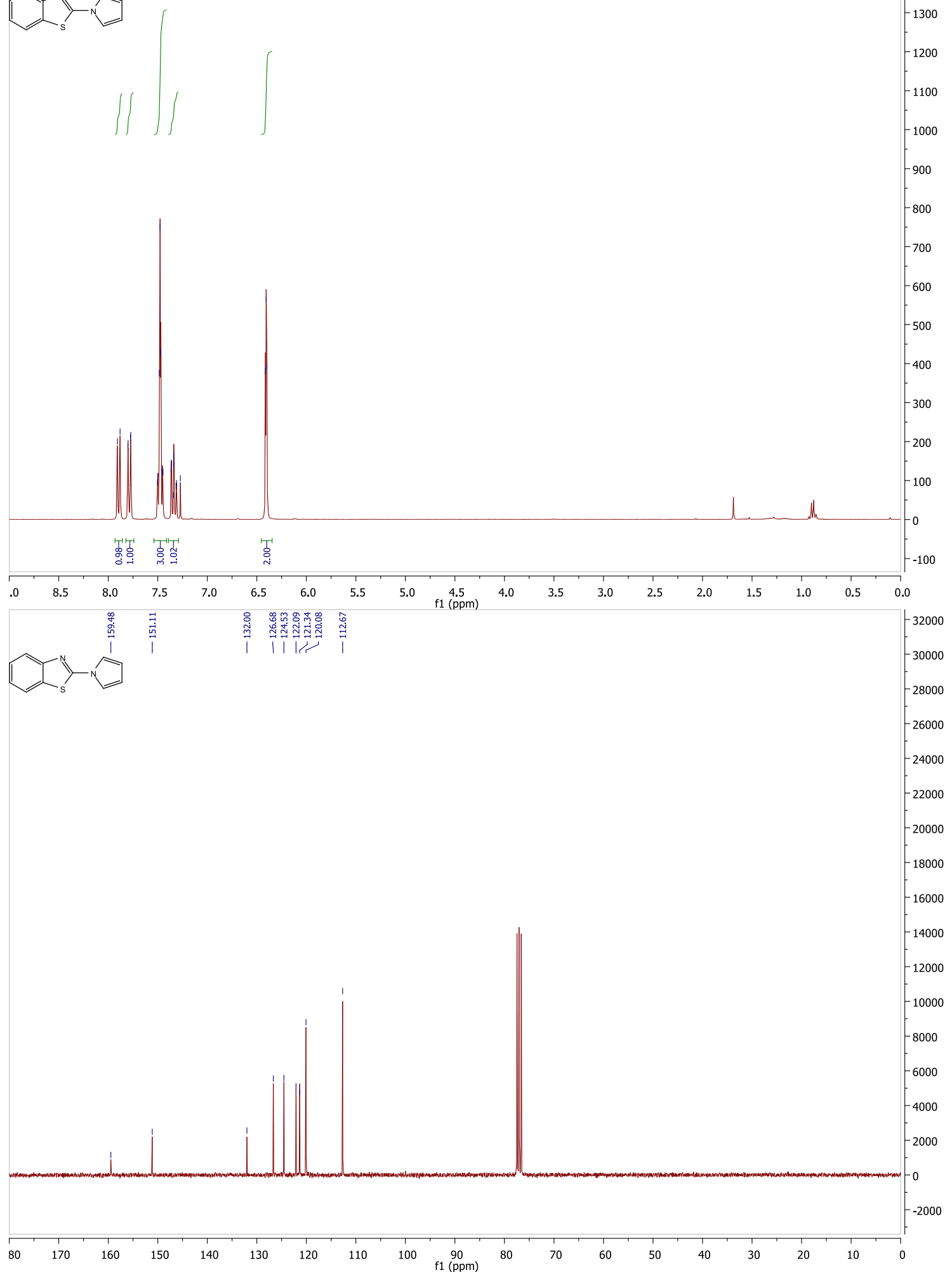


2-(2,4-dimethyl-1H-pyrrol-1-yl)benzo[d]thiazole (4ak)

${ }^{1} \mathrm{H} \mathrm{NMR}\left(300 \mathrm{MHz}, \mathrm{CDCl}_{3}\right)$ and ${ }^{13} \mathrm{C} \mathrm{NMR}\left(75 \mathrm{MHz}, \mathrm{CDCl}_{3}\right)$

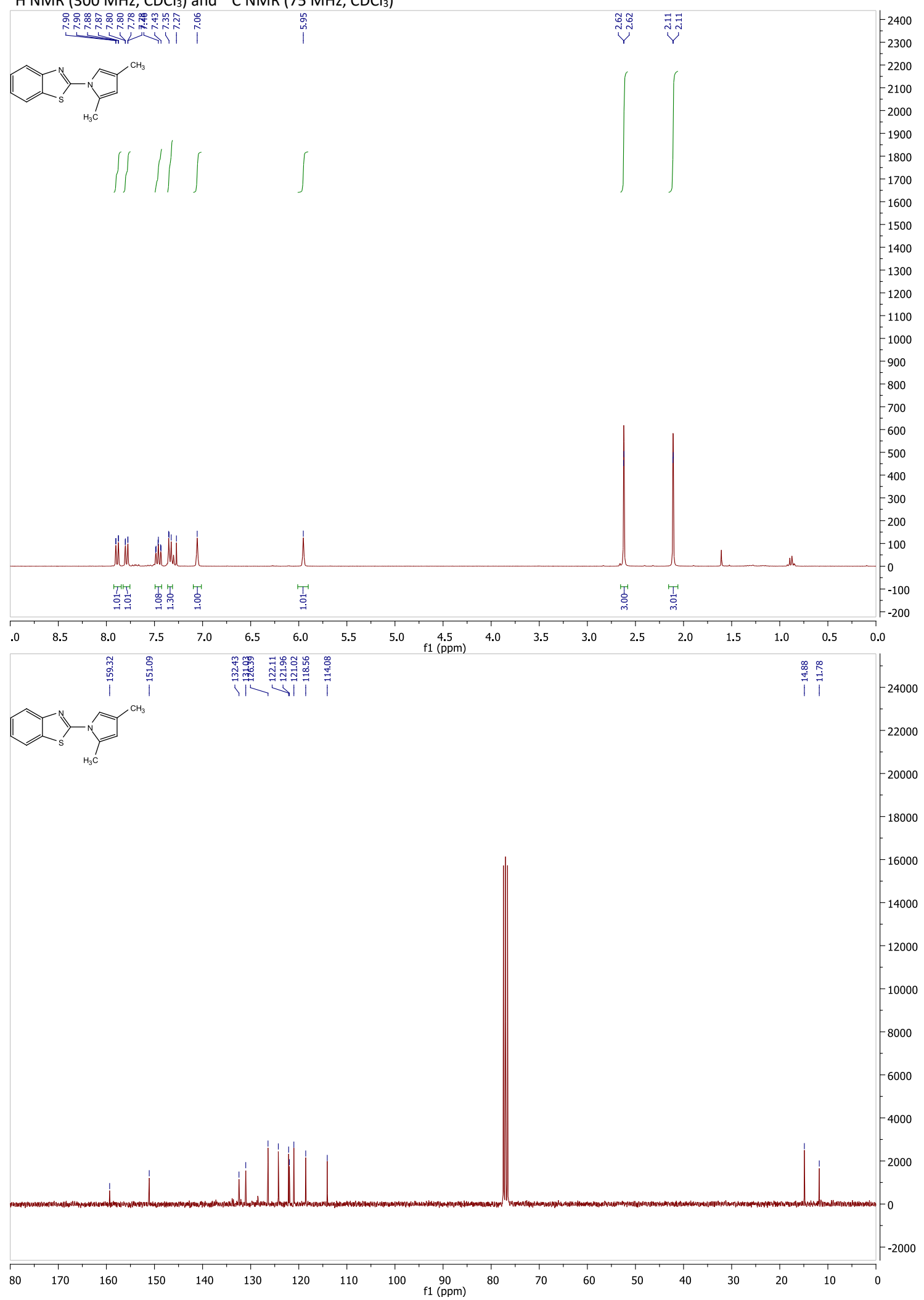



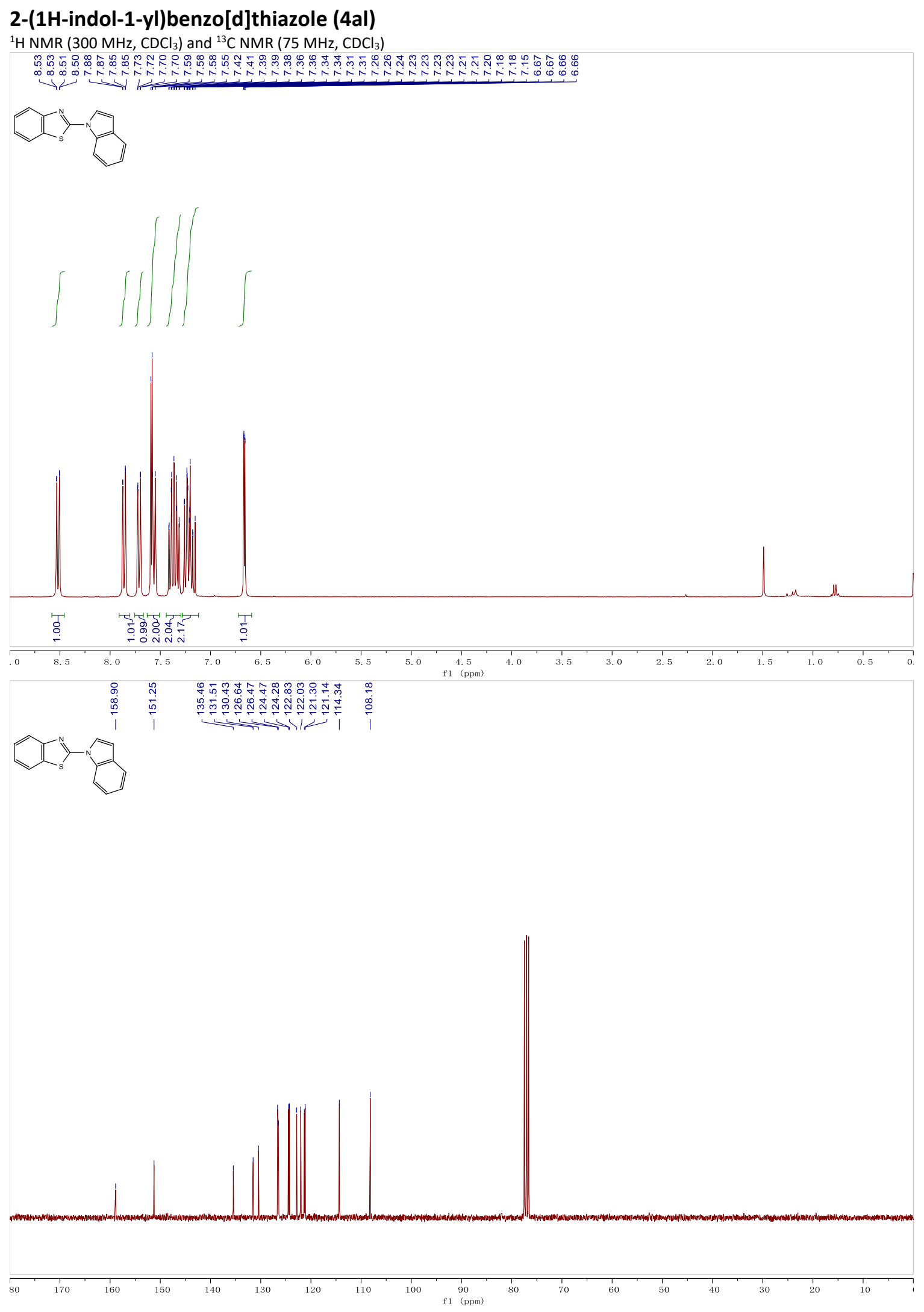

S81 
2-(2-methyl-1H-indol-1-yl)benzo[d]thiazole (4am)

${ }^{1} \mathrm{H} \mathrm{NMR}\left(300 \mathrm{MHz}, \mathrm{CDCl}_{3}\right)$ and ${ }^{13} \mathrm{C} \mathrm{NMR}\left(75 \mathrm{MHz}, \mathrm{CDCl}_{3}\right)$

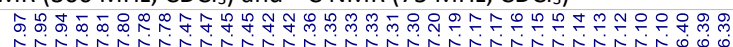
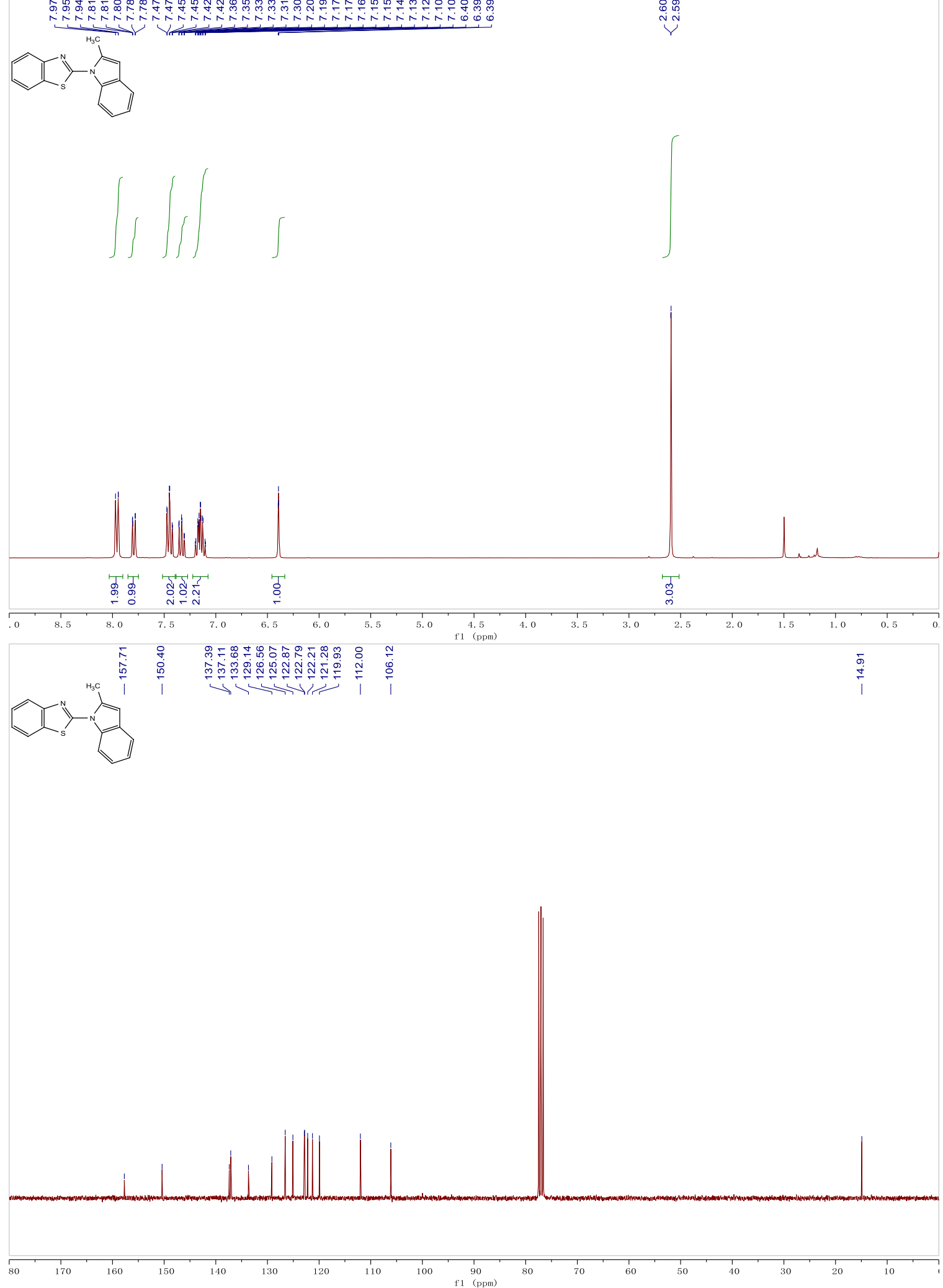


\section{2-(5-bromo-1H-indol-1-yl)benzo[d]thiazole (4an)}

${ }^{1} \mathrm{H} \mathrm{NMR}\left(300 \mathrm{MHz}, \mathrm{CDCl}_{3}\right)$ and ${ }^{13} \mathrm{C} \mathrm{NMR}\left(75 \mathrm{MHz}, \mathrm{CDCl}_{3}\right)$

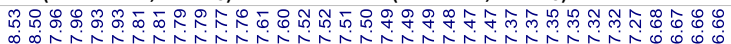
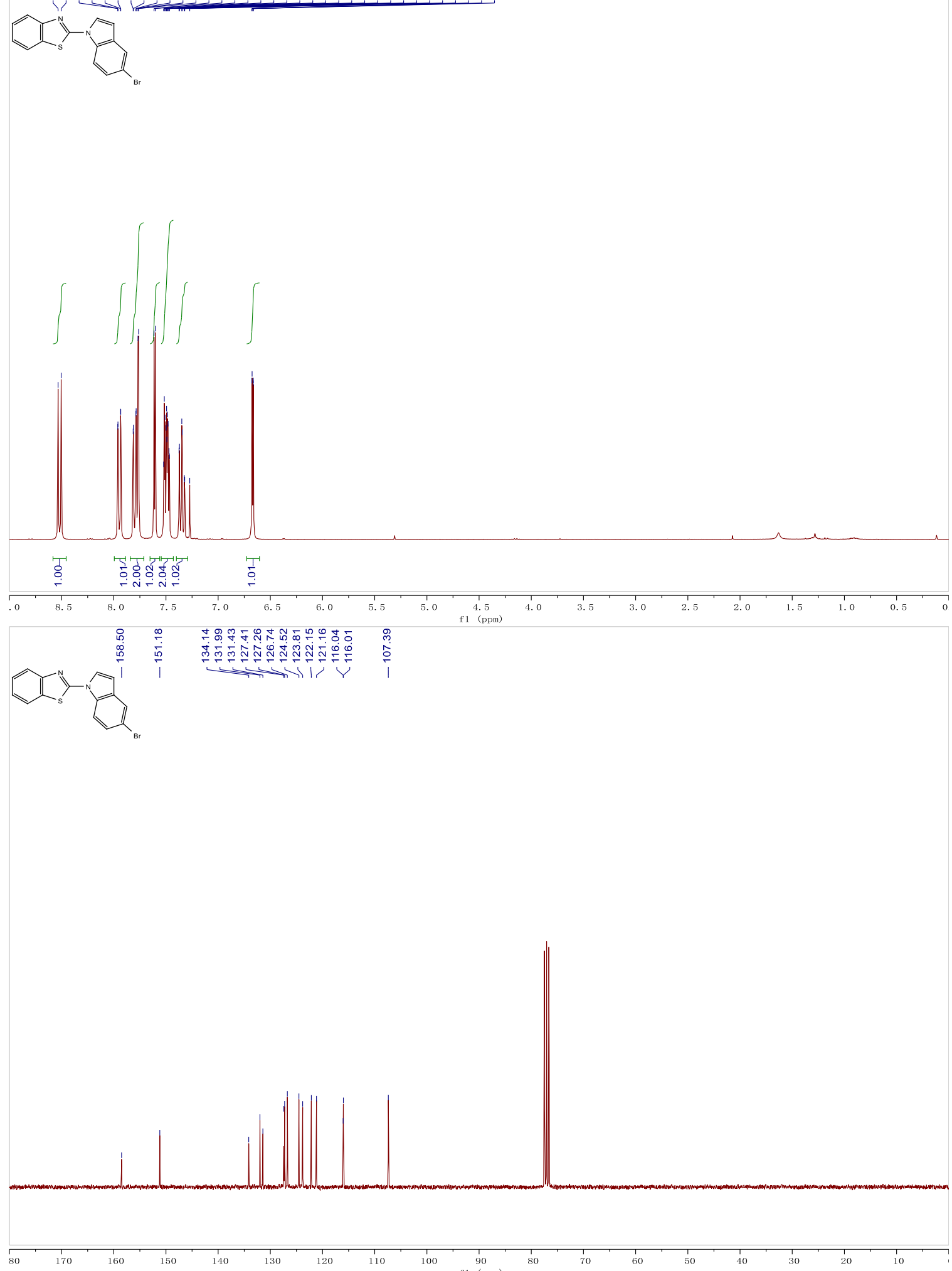
1-(benzo[d]thiazol-2-yl)-1H-indole-4-carbonitrile (4ao)

${ }^{1} \mathrm{H} \mathrm{NMR}\left(300 \mathrm{MHz}, \mathrm{CDCl}_{3}\right)$ and ${ }^{13} \mathrm{C} \mathrm{NMR}\left(75 \mathrm{MHz}, \mathrm{CDCl}_{3}\right)$

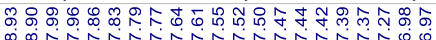
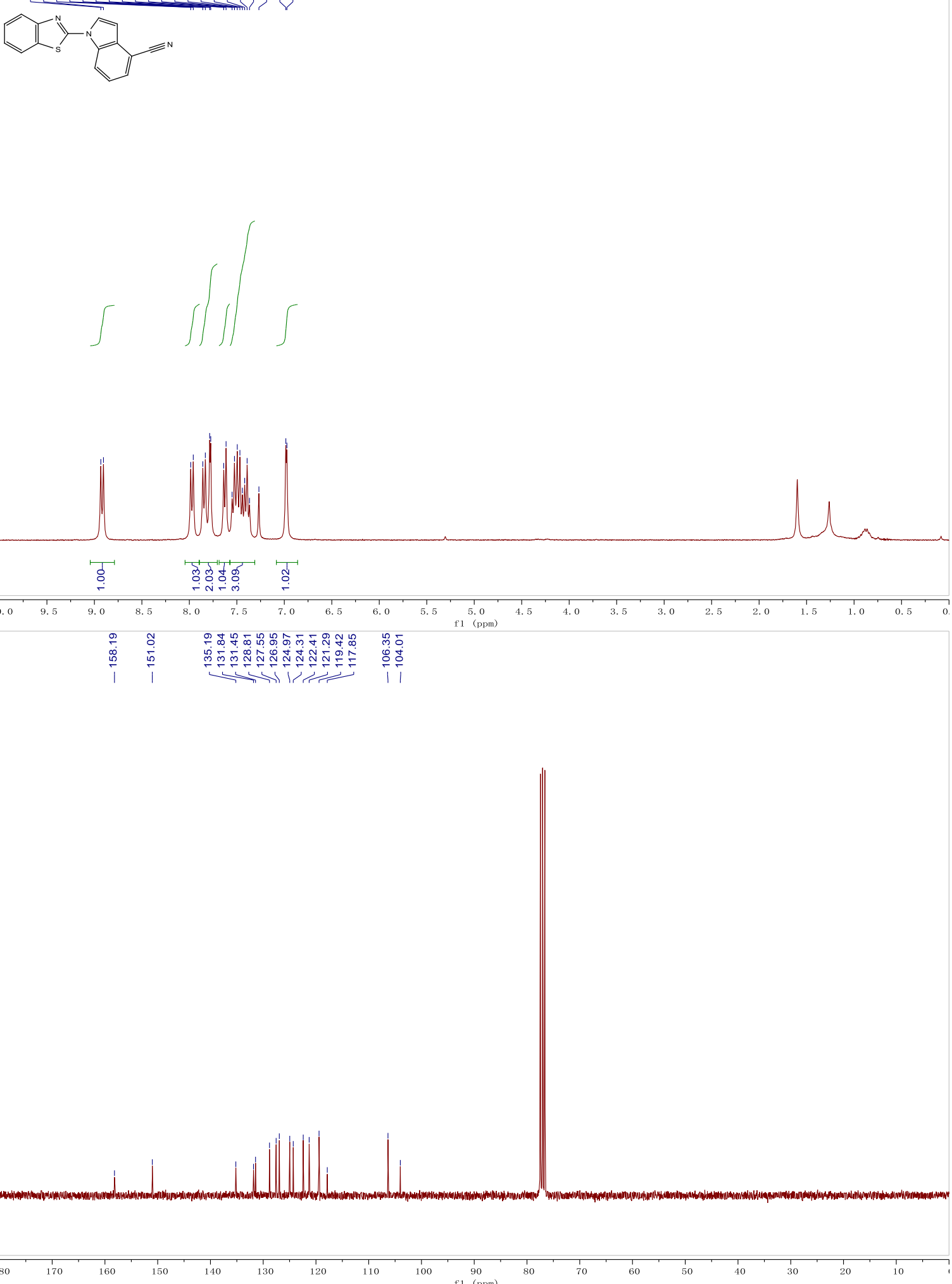
2-(5-nitro-1H-indol-1-yl)benzo[d]thiazole (4ap)

${ }^{1} \mathrm{H}$ NMR $\left(300 \mathrm{MHz}, \mathrm{CDCl}_{3}\right)$ and ${ }^{13} \mathrm{C} \mathrm{NMR}\left(75 \mathrm{MHz}, \mathrm{CDCl}_{3}\right)$

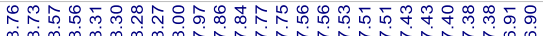

(

$2 \overbrace{}^{N}$

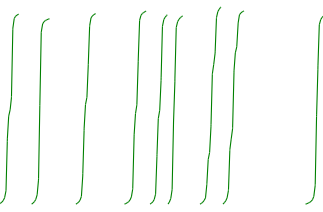

(4)

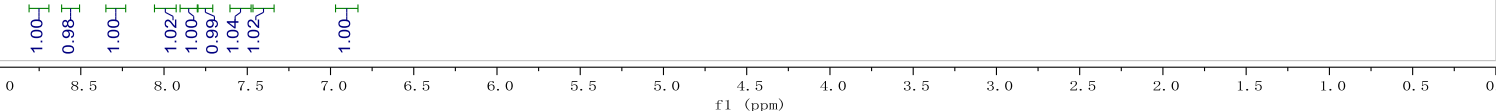

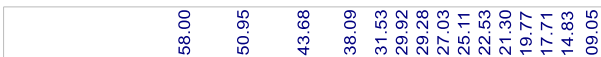

Q1<smiles>C1CC2CCCC2C1</smiles>
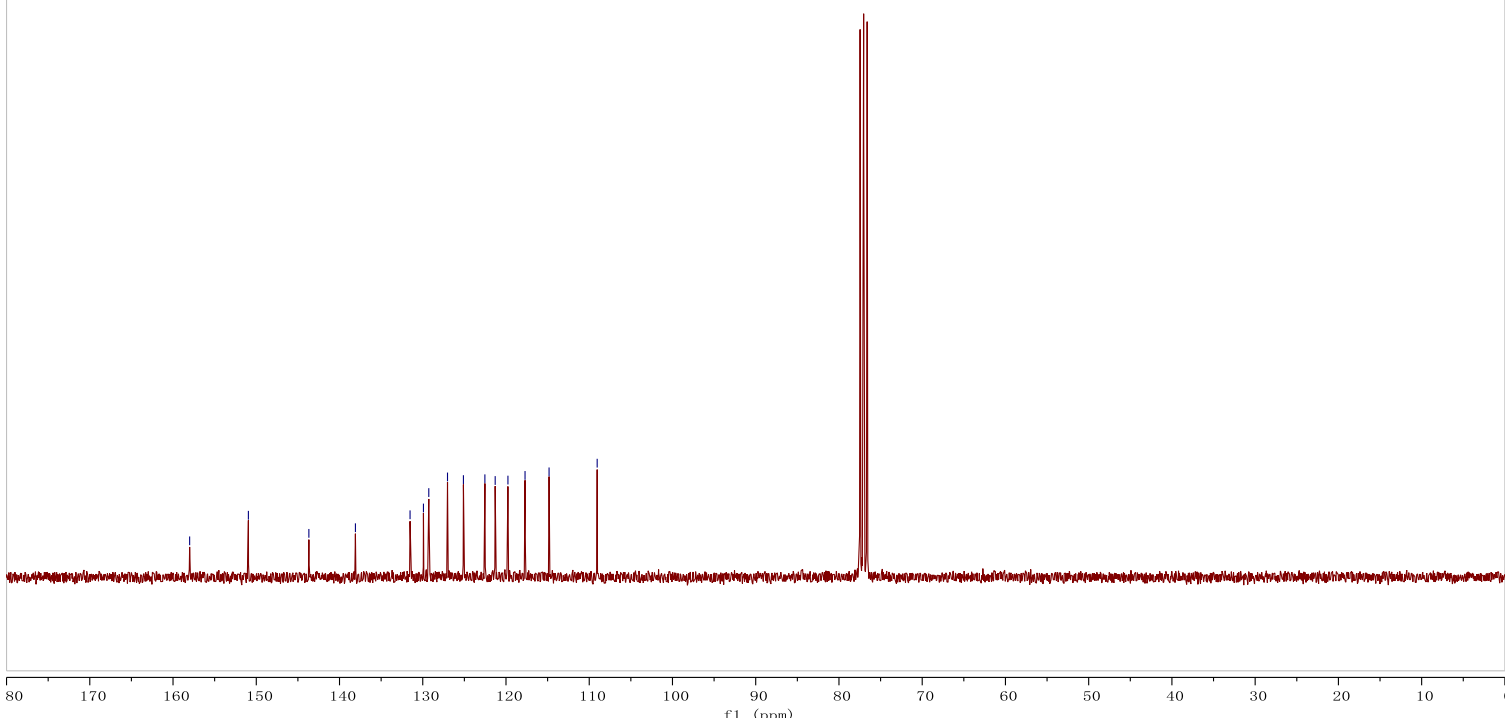
2-(1H-benzo[d]imidazol-1-yl)benzo[d]thiazole (4aq)

${ }^{1} \mathrm{H} \mathrm{NMR}\left(300 \mathrm{MHz}, \mathrm{CDCl}_{3}\right)$ and ${ }^{13} \mathrm{C} \mathrm{NMR}\left(75 \mathrm{MHz}, \mathrm{CDCl}_{3}\right)$

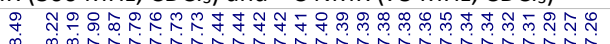

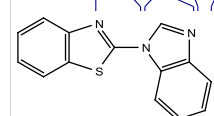
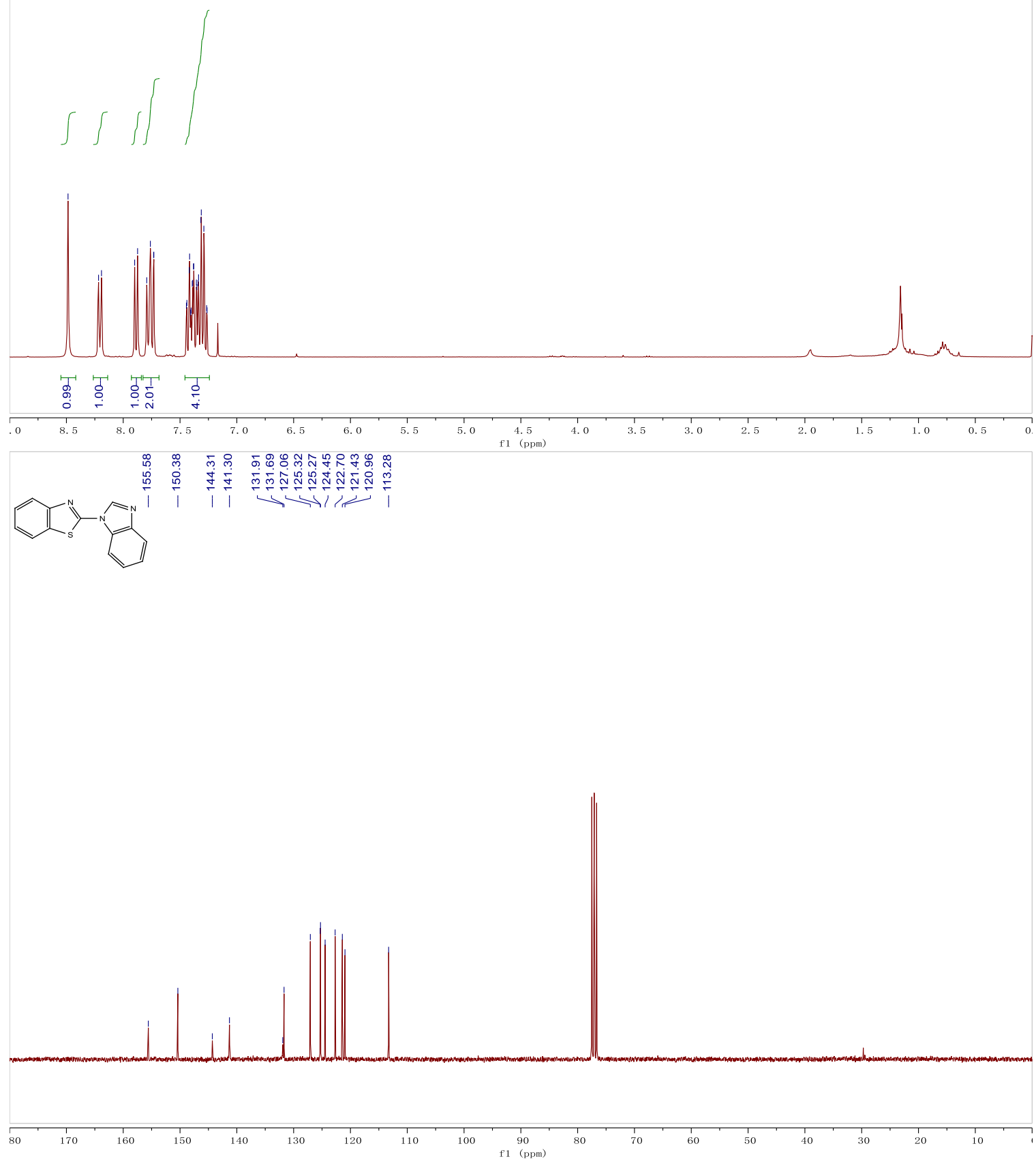
2-(1H-pyrazol-1-yl)benzo[d]thiazole (4ar)

${ }^{1} \mathrm{H} \mathrm{NMR}\left(300 \mathrm{MHz}, \mathrm{CDCl}_{3}\right)$ and ${ }^{13} \mathrm{C} \mathrm{NMR}\left(75 \mathrm{MHz}, \mathrm{CDCl}_{3}\right)$

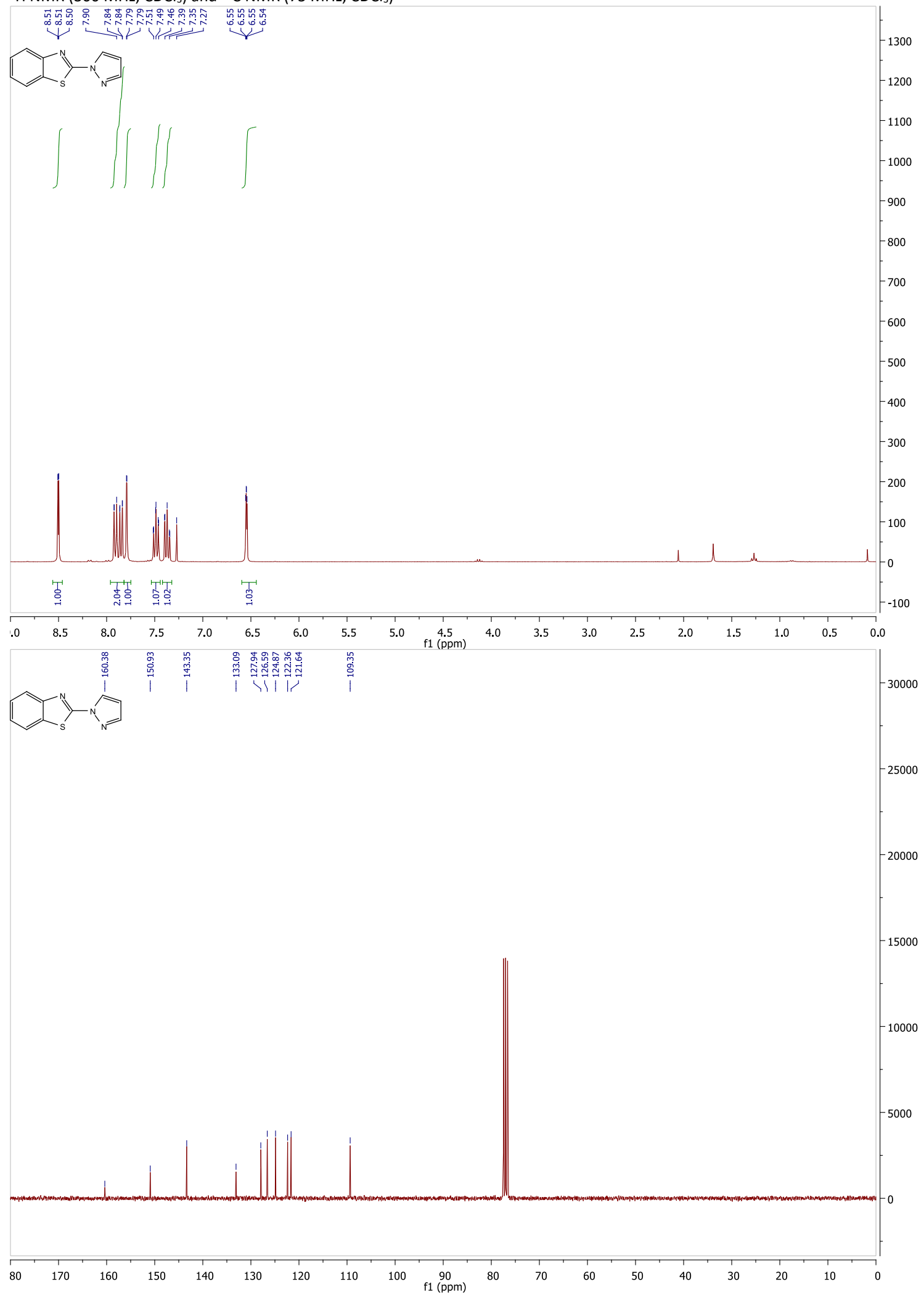


2-(1H-imidazol-1-yl)benzo[d]thiazole (4as)

${ }^{1} \mathrm{H} \mathrm{NMR}\left(300 \mathrm{MHz}, \mathrm{CDCl}_{3}\right)$ and ${ }^{13} \mathrm{C} \mathrm{NMR}\left(75 \mathrm{MHz}, \mathrm{CDCl}_{3}\right)$

管
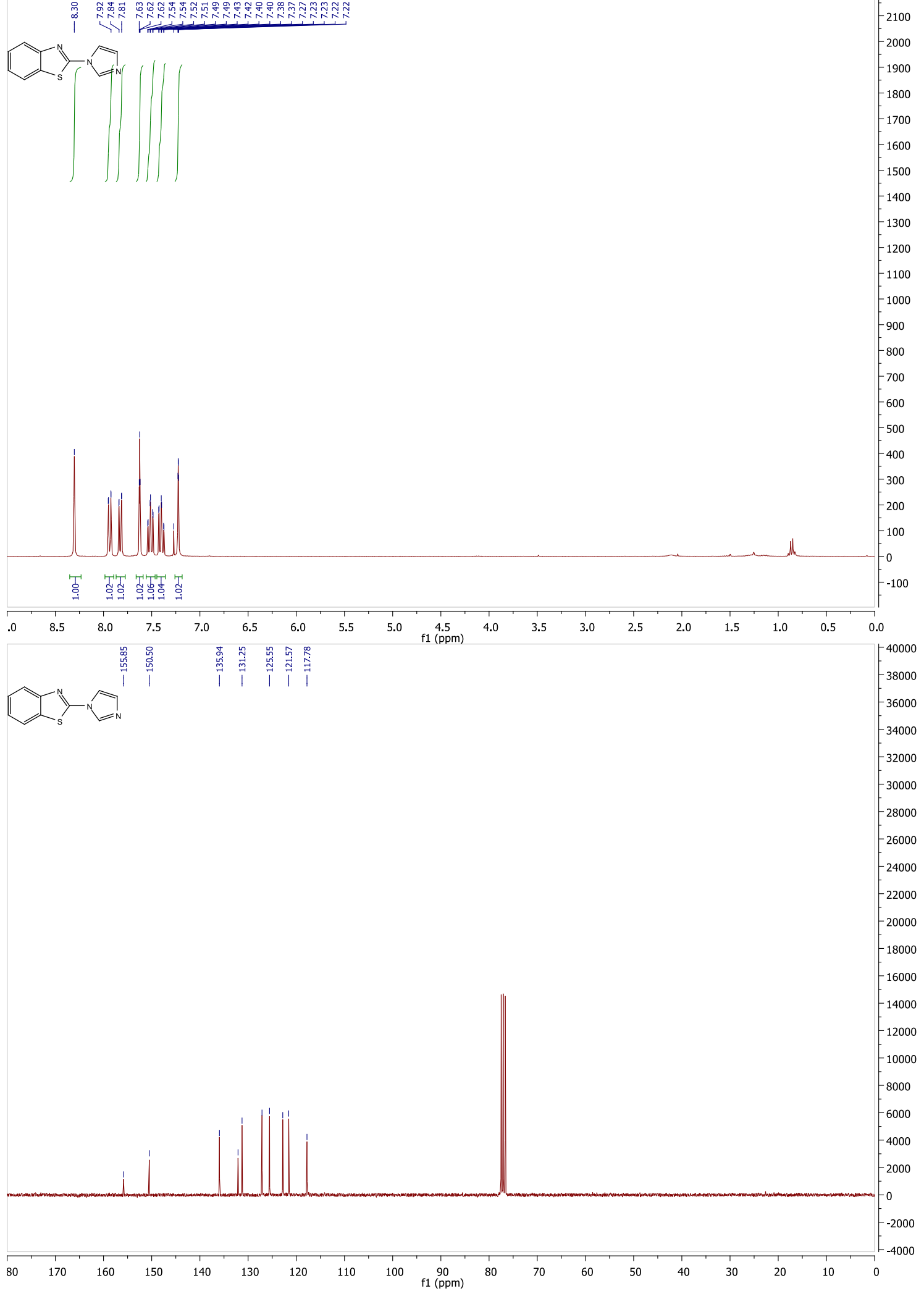
2-(2-ethyl-4-methyl-1H-imidazol-1-yl)benzo[d]thiazole (4at)

${ }^{1} \mathrm{H} \mathrm{NMR}\left(300 \mathrm{MHz}, \mathrm{CDCl}_{3}\right)$ and ${ }^{13} \mathrm{C} \mathrm{NMR}\left(75 \mathrm{MHz}, \mathrm{CDCl}_{3}\right)$

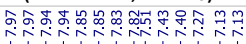
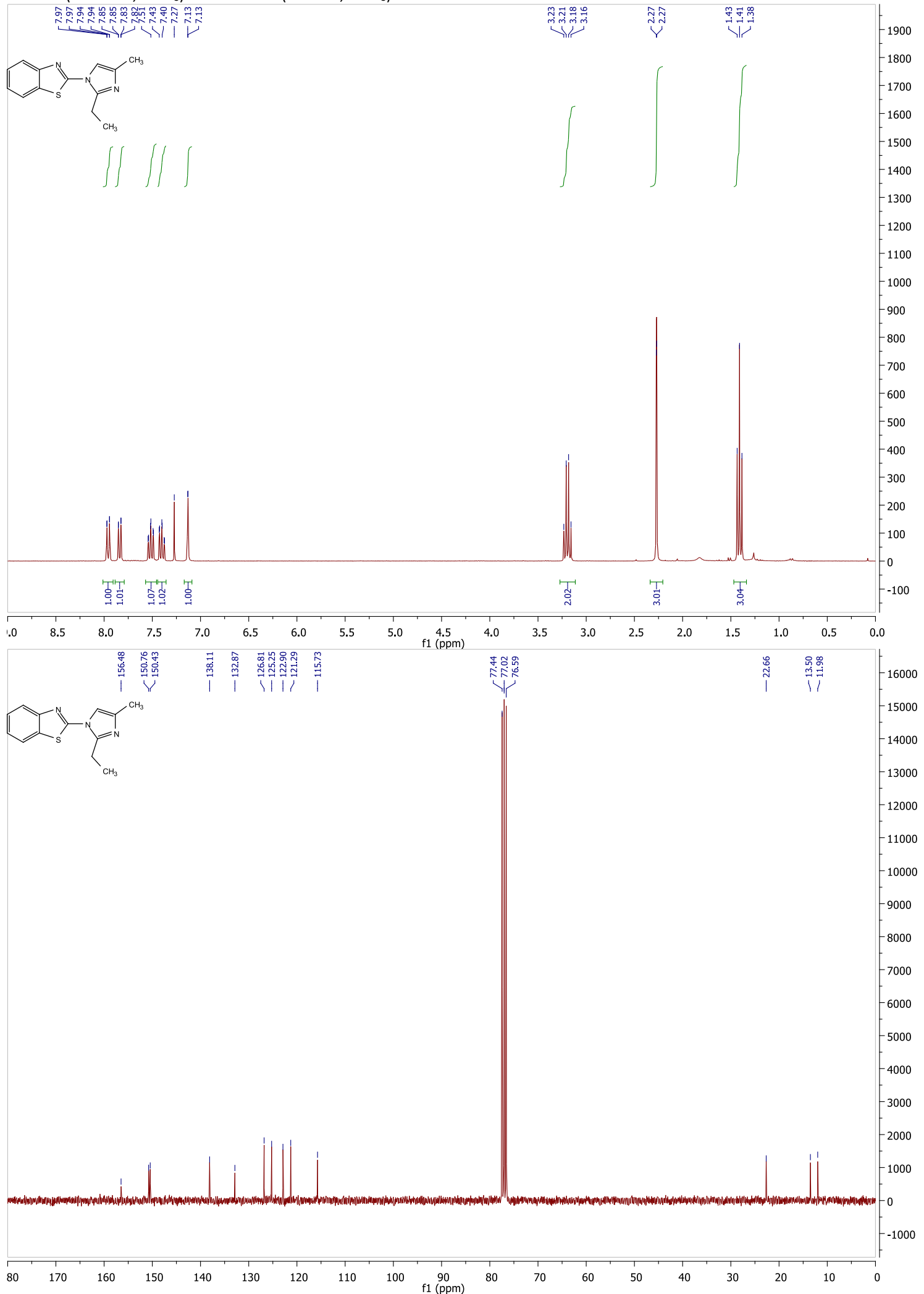


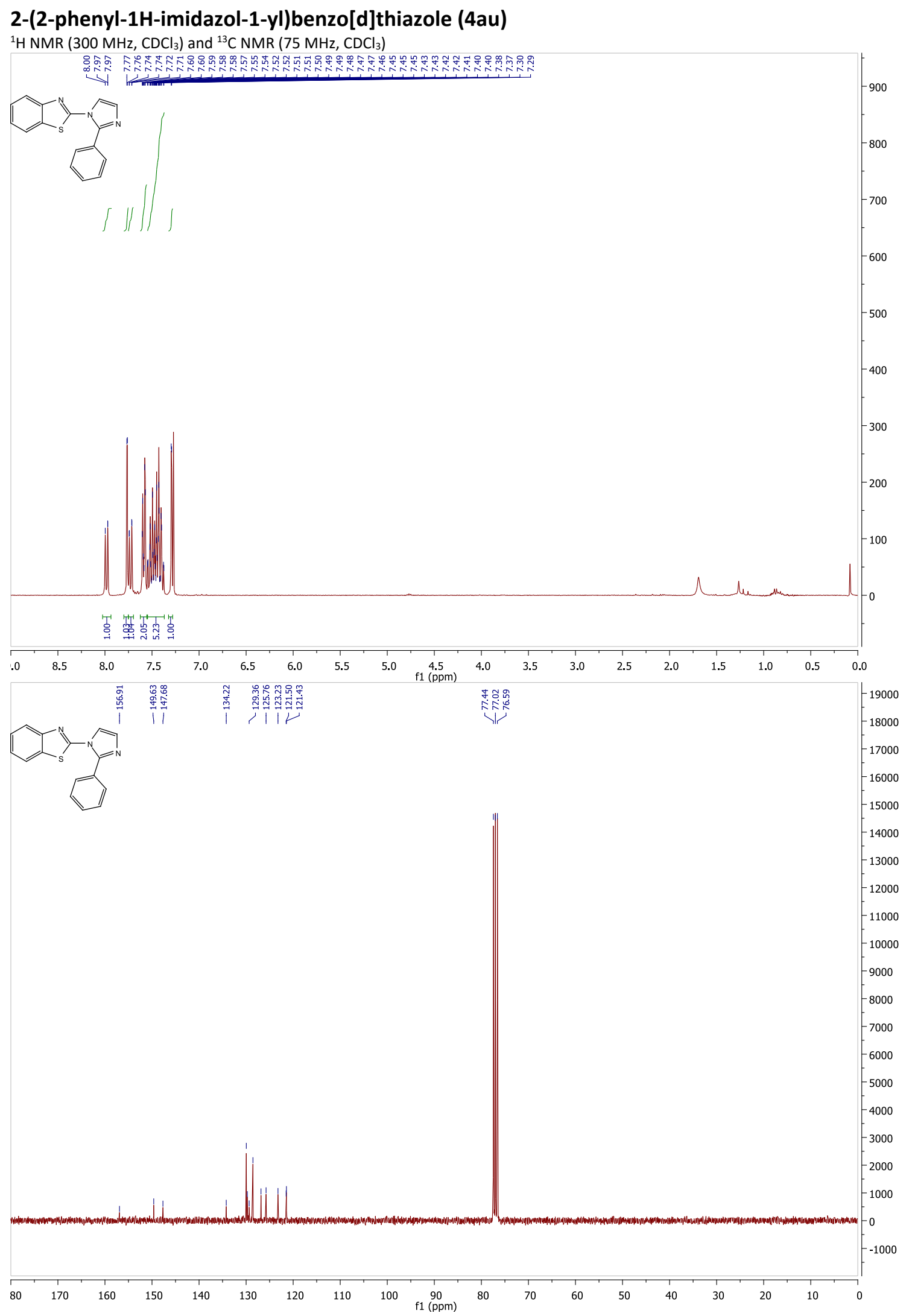


2-(4-methoxyphenoxy)-6-methylbenzo[d]thiazole (4av)

${ }^{1} \mathrm{H}$ NMR $\left(300 \mathrm{MHz}, \mathrm{CDCl}_{3}\right)$ and ${ }^{13} \mathrm{C} \mathrm{NMR}\left(75 \mathrm{MHz}, \mathrm{CDCl}_{3}\right)$

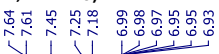
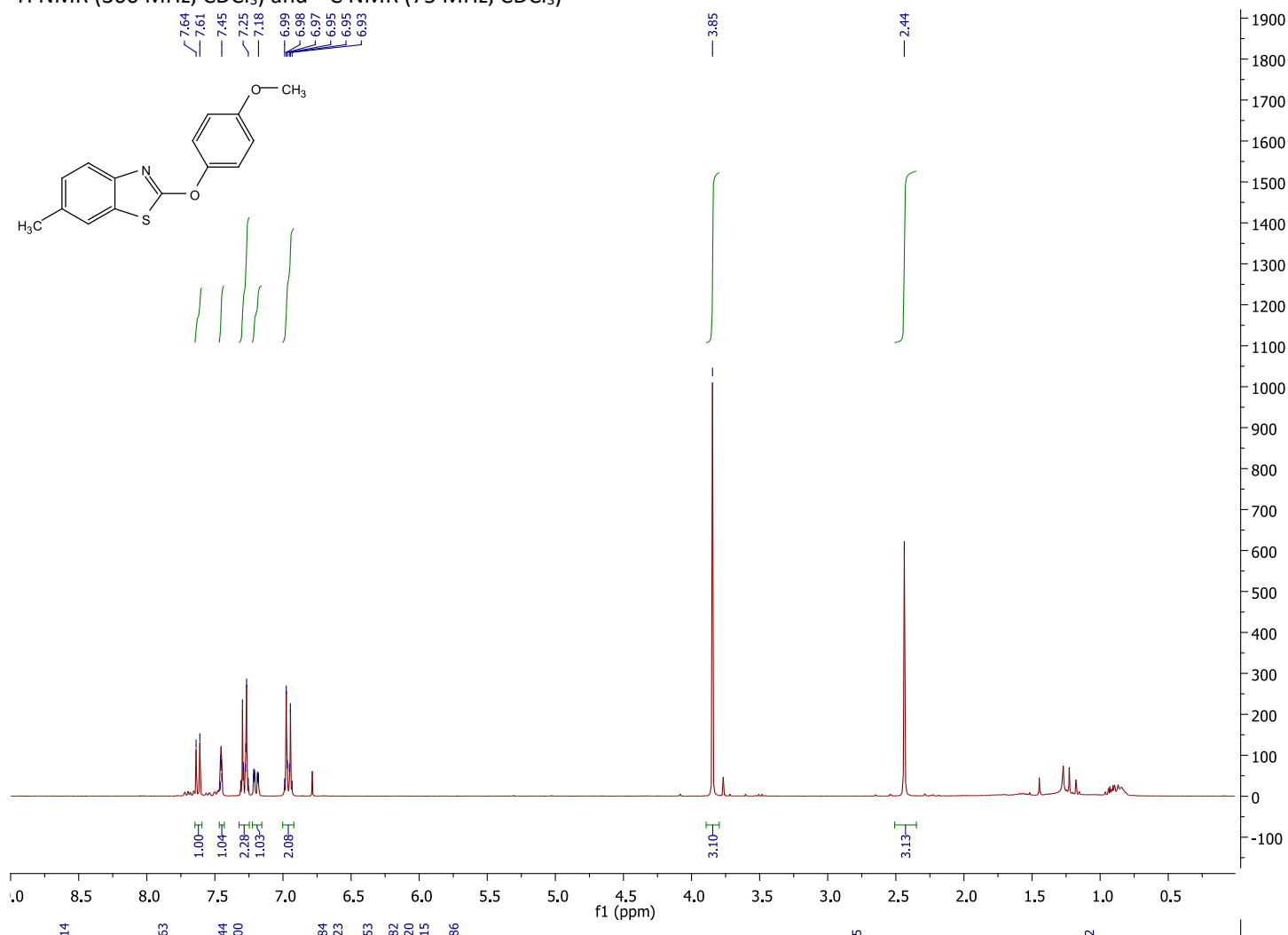

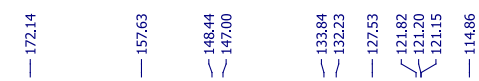
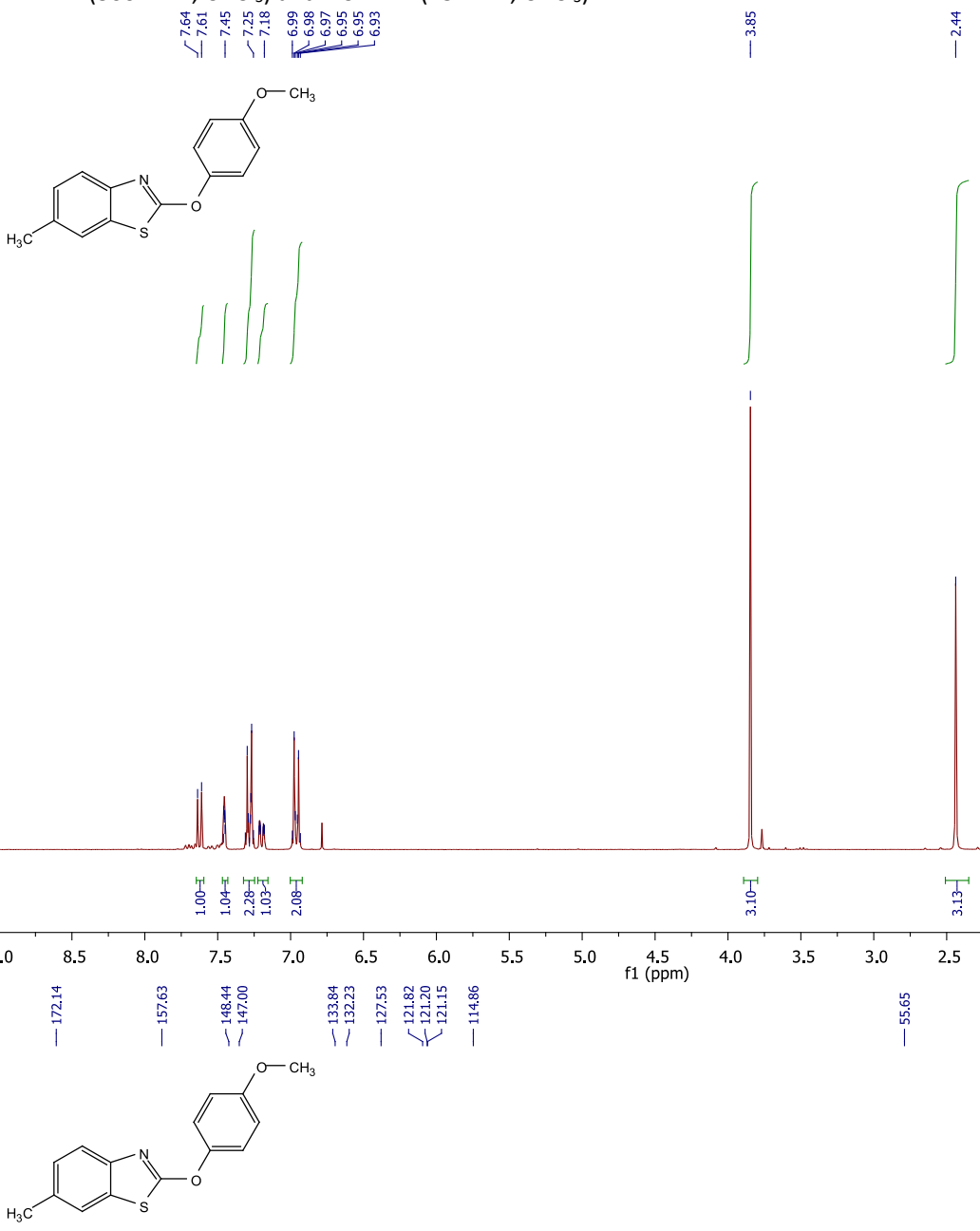

\begin{tabular}{l}
-1900 \\
-1800 \\
-1700 \\
-1600 \\
-1500 \\
-1400 \\
-1300 \\
-1200 \\
-1100 \\
-1000 \\
-900 \\
-800 \\
-700 \\
-600 \\
-500 \\
-400 \\
-300 \\
-200 \\
-100 \\
-0 \\
-100 \\
\hline
\end{tabular}

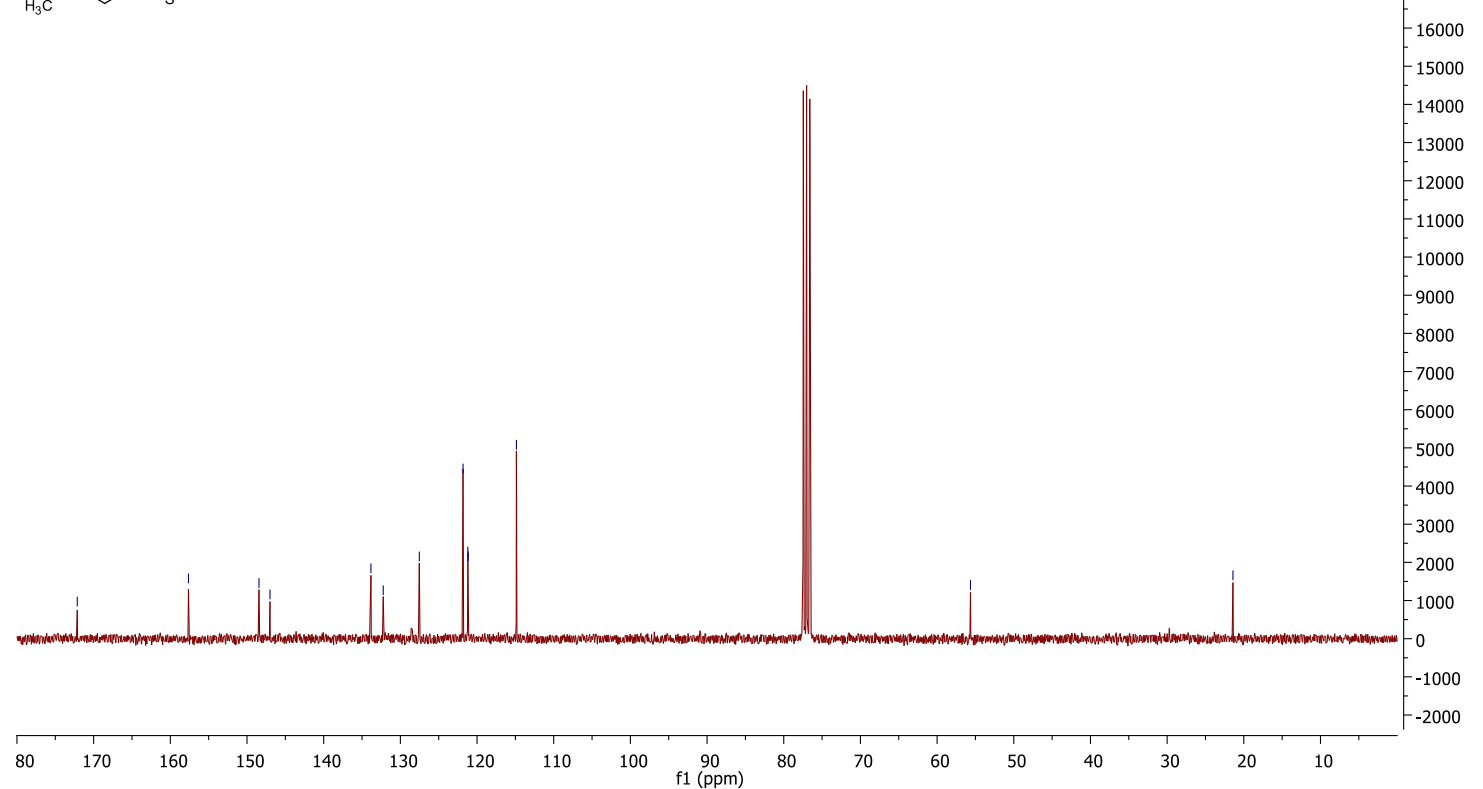




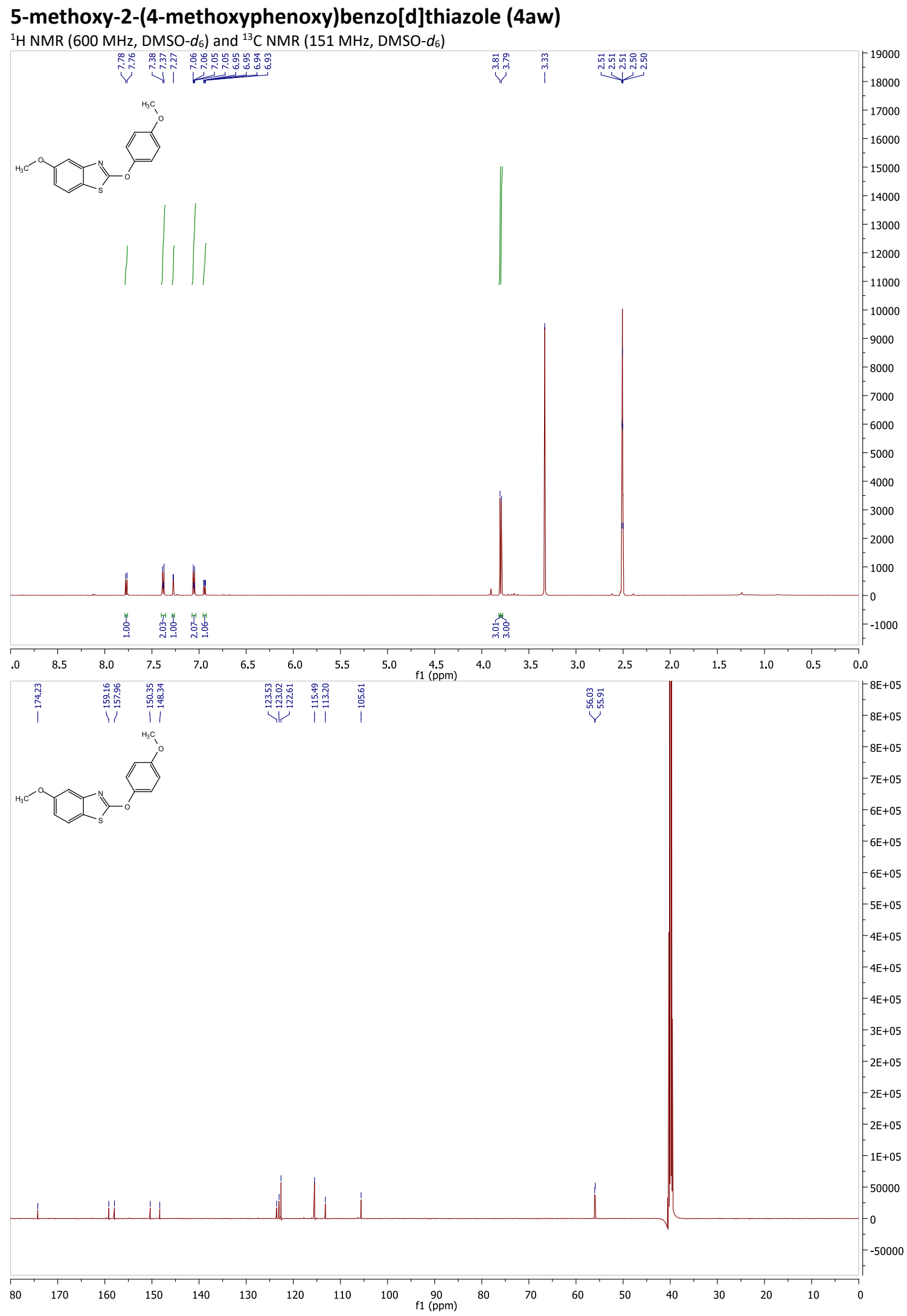


6-fluoro-2-(4-methoxyphenoxy)benzo[d]thiazole (4ax)

${ }^{1} \mathrm{H} \mathrm{NMR}\left(300 \mathrm{MHz}, \mathrm{CDCl}_{3}\right)$ and ${ }^{13} \mathrm{C} \mathrm{NMR}\left(75 \mathrm{MHz}, \mathrm{CDCl}_{3}\right)$

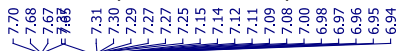
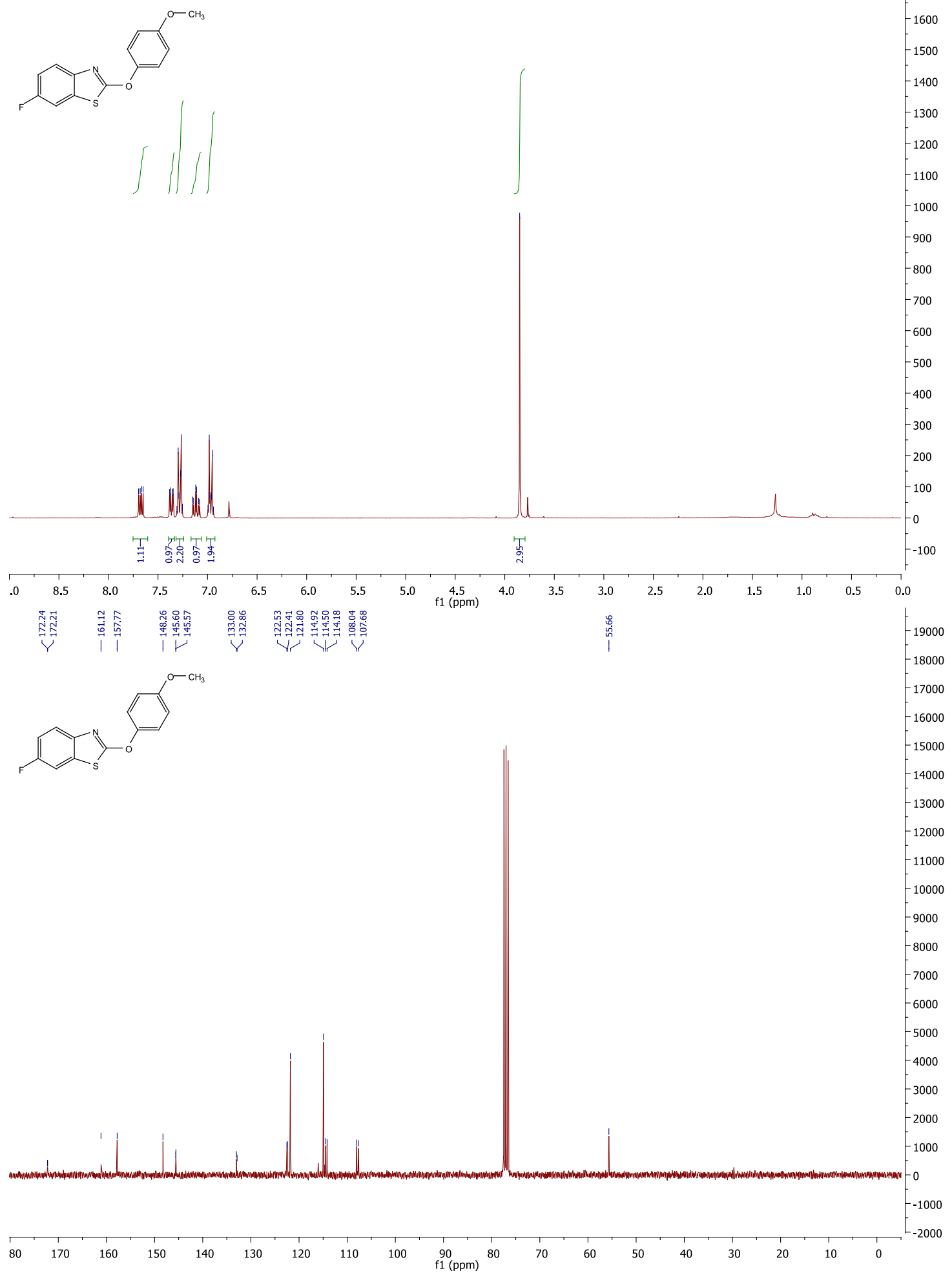
7-bromo-2-(4-methoxyphenoxy)benzo[d]thiazole (4ay)

${ }^{1} \mathrm{H} \mathrm{NMR}\left(300 \mathrm{MHz}, \mathrm{CDCl}_{3}\right)$ and ${ }^{13} \mathrm{C} \mathrm{NMR}\left(75 \mathrm{MHz}, \mathrm{CDCl}_{3}\right)$

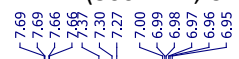
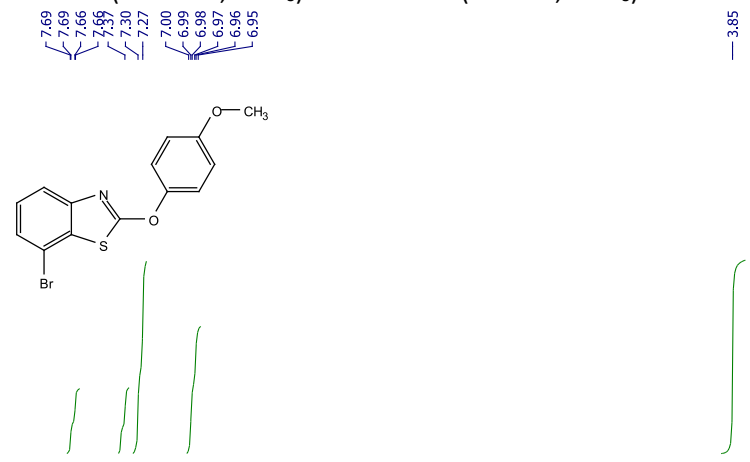

2600

2400

2200

2000

1800

$-1600$

$-1400$

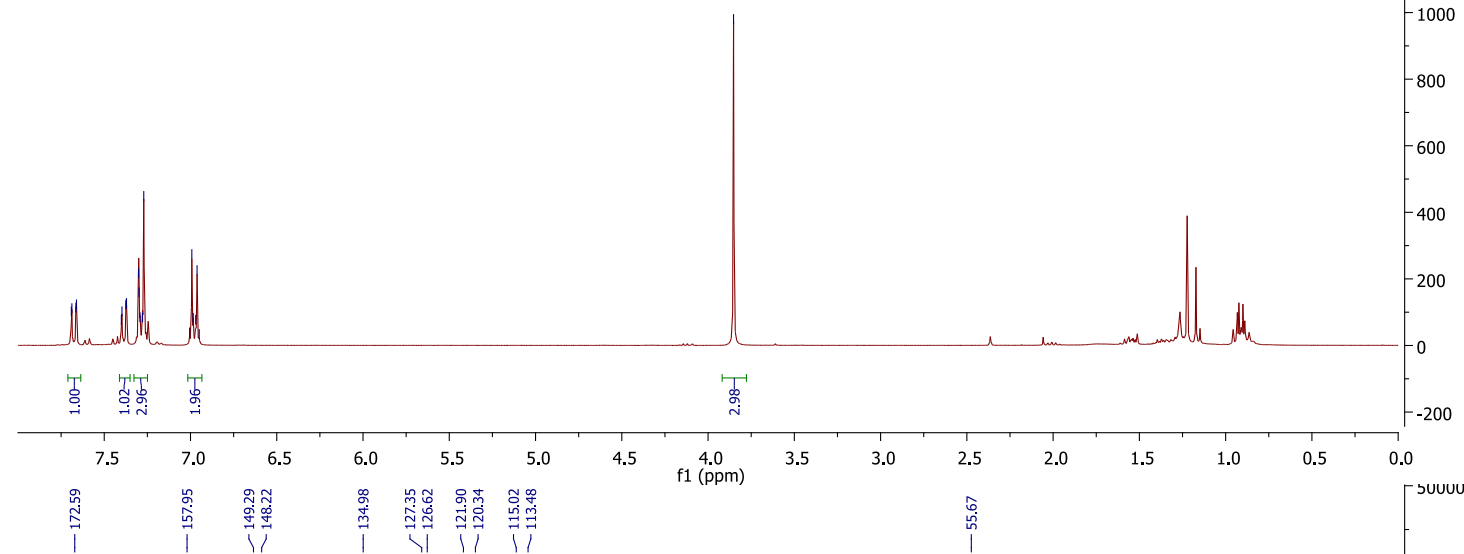

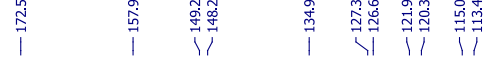

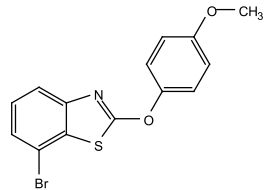

$-45000$

40000

35000

30000

25000

20000

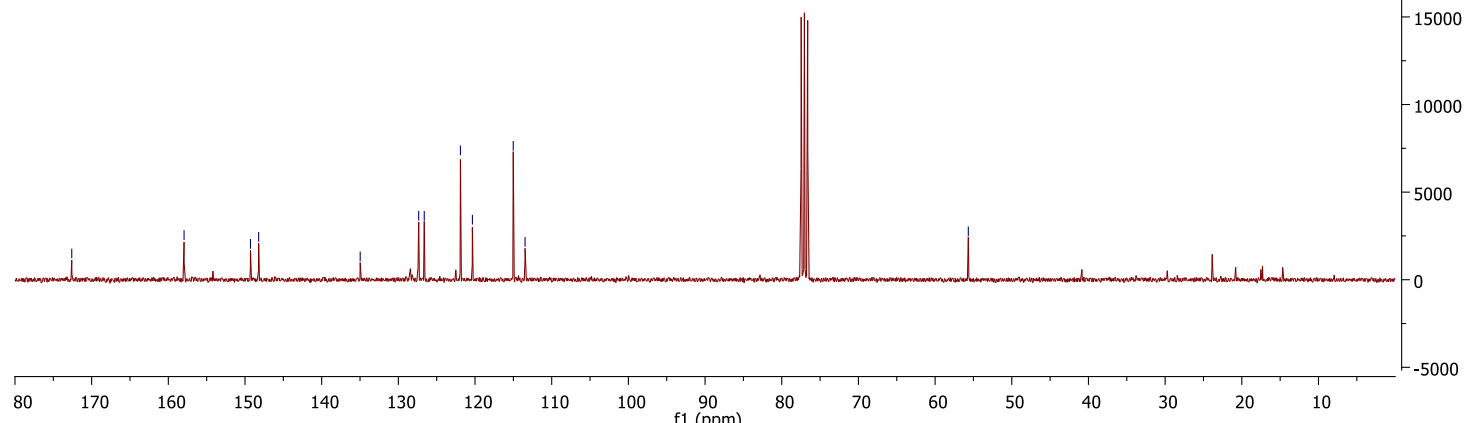


5-bromo-2-(4-methoxyphenoxy)benzo[d]thiazole (4az)

${ }^{1} \mathrm{H}$ NMR $\left(600 \mathrm{MHz}\right.$, DMSO- $\left.d_{6}\right)$ and ${ }^{13} \mathrm{C}$ NMR $\left(151 \mathrm{MHz}\right.$, DMSO- $\left.d_{6}\right)$

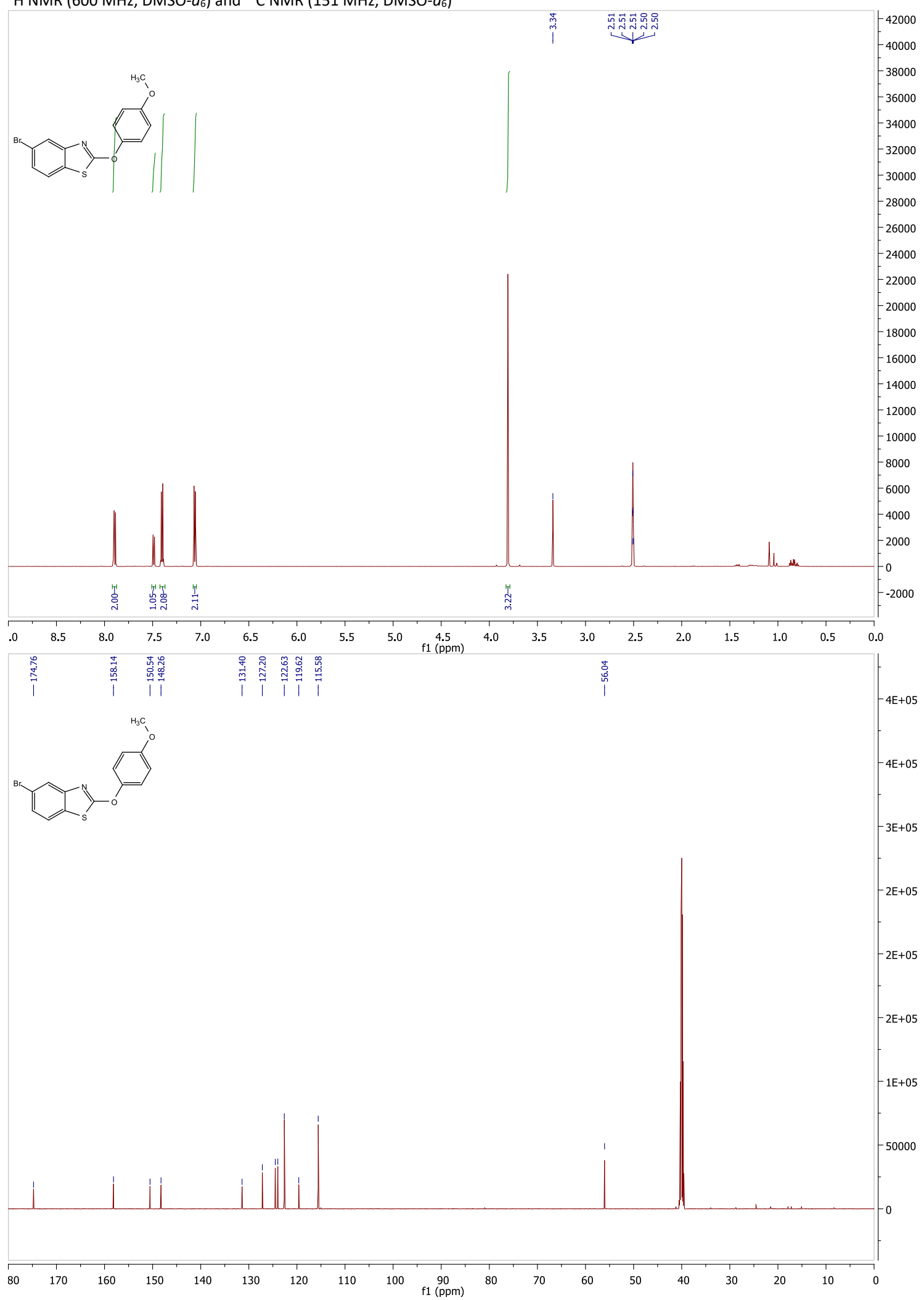


6-chloro-2-(4-methoxyphenoxy)benzo[d]thiazole (4ba)

${ }^{1} \mathrm{H} \mathrm{NMR}\left(300 \mathrm{MHz}, \mathrm{CDCl}_{3}\right)$ and ${ }^{13} \mathrm{C} \mathrm{NMR}\left(75 \mathrm{MHz}, \mathrm{CDCl}_{3}\right)$ 谒

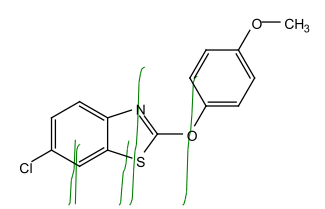

\section{$\stackrel{\substack{\infty \\ \text { i }}}{\mid \infty}$}
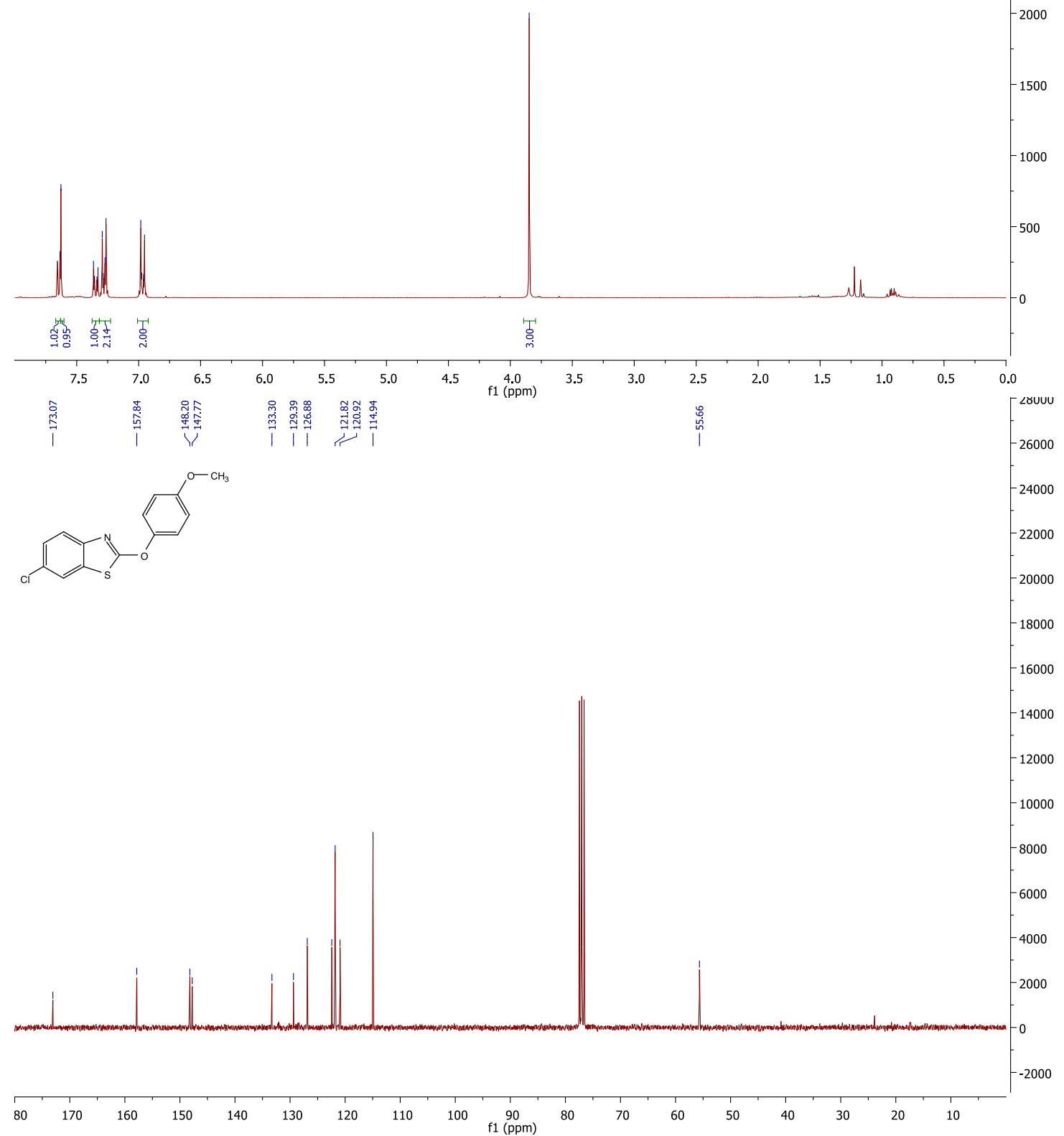
2-(4-methoxyphenoxy)benzo[d]thiazole-5-carbonitrile (4bb)

${ }^{1} \mathrm{H}$ NMR $\left(400 \mathrm{MHz}, \mathrm{CDCl}_{3}\right)$ and $\left.{ }^{13} \mathrm{C} \mathrm{NMR} \mathrm{(101} \mathrm{MHz,} \mathrm{CDCl}_{3}\right)$

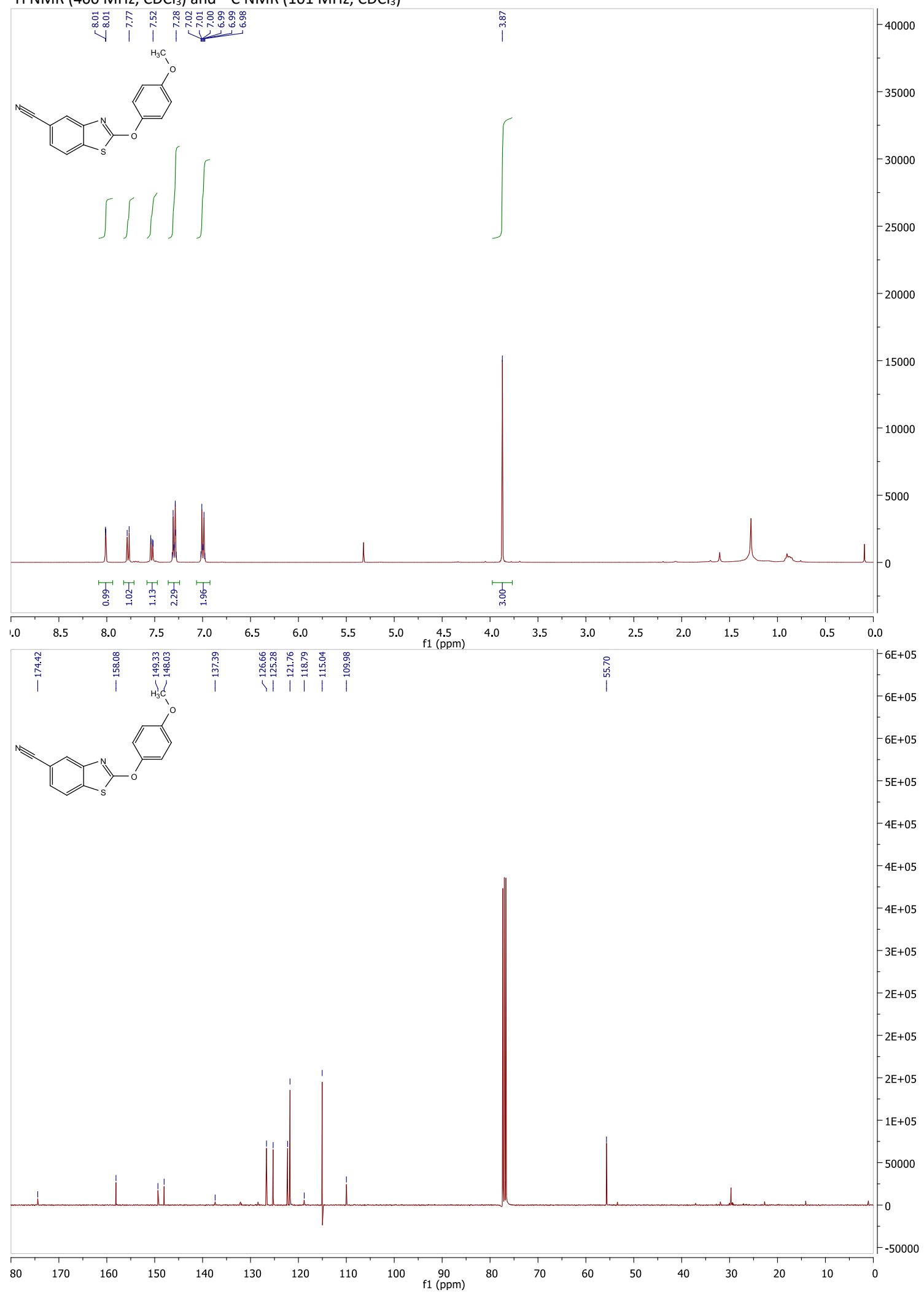


2-(4-methoxyphenoxy)benzo[d]thiazole-6-carbonitrile (4bc)

${ }^{1} \mathrm{H} \mathrm{NMR}\left(300 \mathrm{MHz}, \mathrm{CDCl}_{3}\right)$ and ${ }^{13} \mathrm{C} \mathrm{NMR}\left(75 \mathrm{MHz}, \mathrm{CDCl}_{3}\right)$

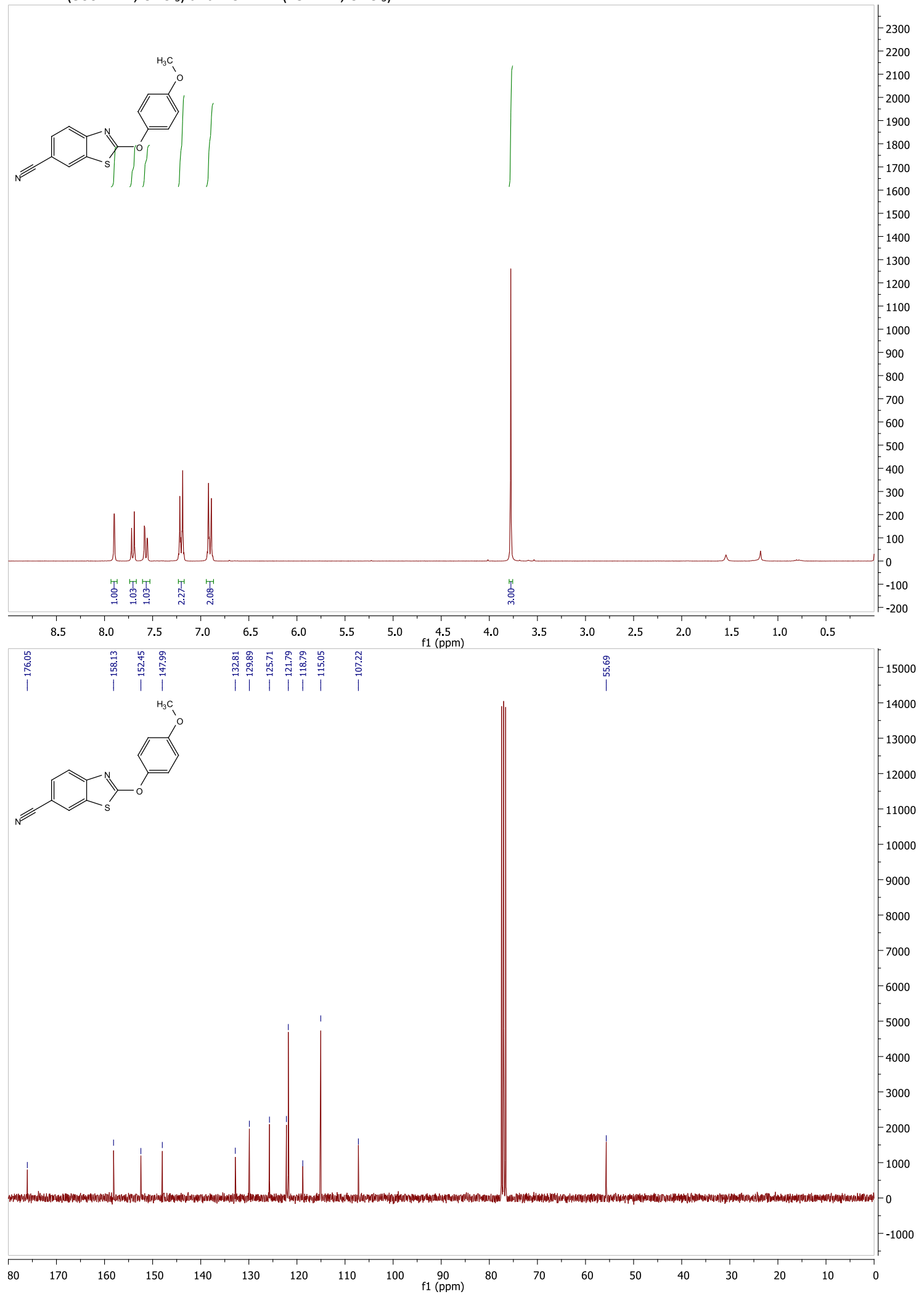




\section{2-(4-methoxyphenoxy)-6-nitrobenzo[d]thiazole (4bd)}

${ }^{1} \mathrm{H} \mathrm{NMR}\left(300 \mathrm{MHz}, \mathrm{CDCl}_{3}\right)$ and ${ }^{13} \mathrm{C} \mathrm{NMR}\left(75 \mathrm{MHz}, \mathrm{CDCl}_{3}\right)$
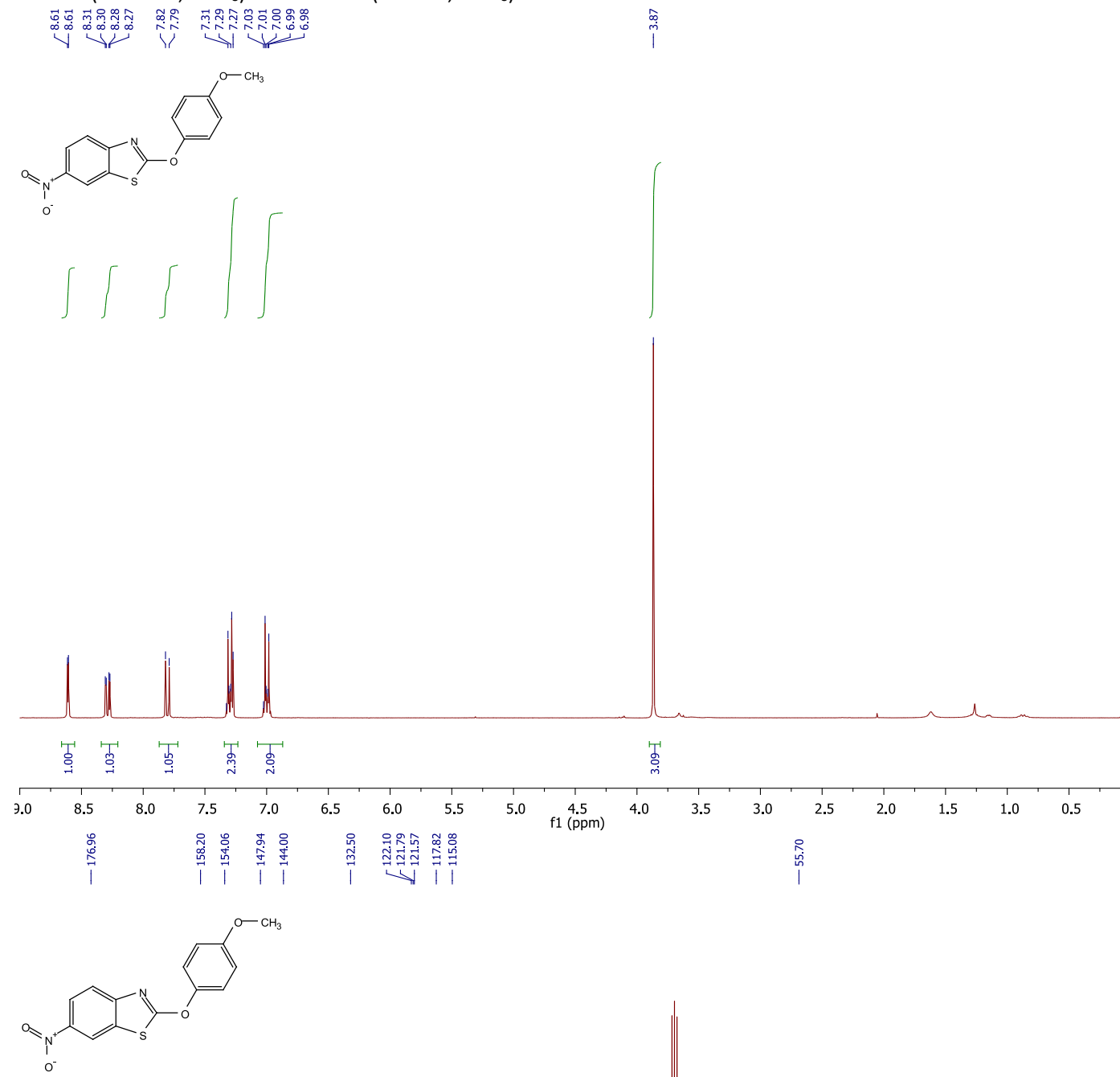


\section{6-methyl-2-(5-nitro-1H-indol-1-yl)benzo[d]thiazole (4be)}

${ }^{1} \mathrm{H}$ NMR $\left(600 \mathrm{MHz}\right.$, DMSO- $\left.d_{6}\right)$ and ${ }^{13} \mathrm{C}$ NMR $\left(151 \mathrm{MHz}\right.$, DMSO- $\left.d_{6}\right)$

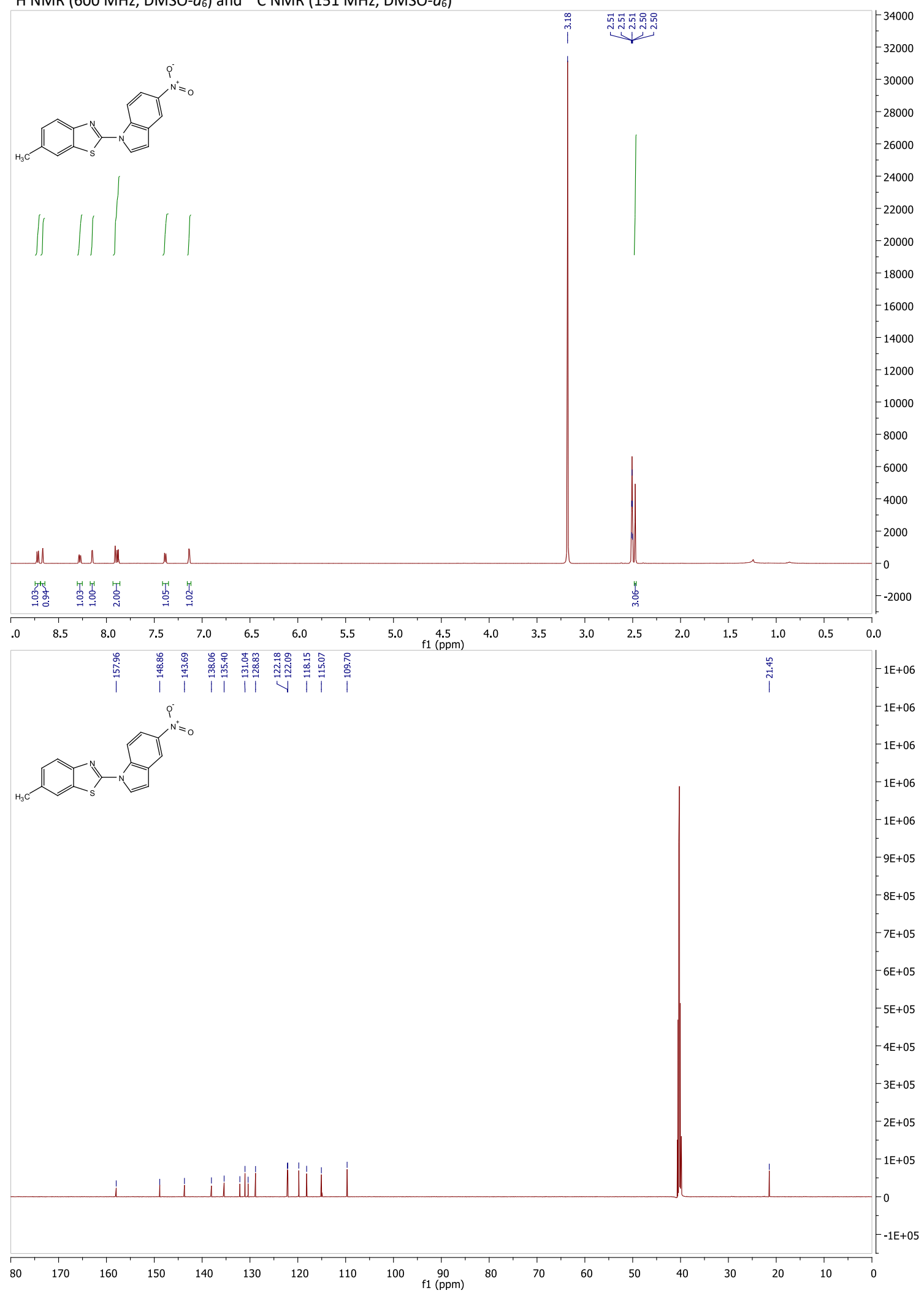




\section{5-methoxy-2-(5-nitro-1H-indol-1-yl)benzo[d]thiazole (4bf)}

${ }^{1} \mathrm{H}$ NMR $\left(600 \mathrm{MHz}\right.$, DMSO- $\left.d_{6}\right)$ and ${ }^{13} \mathrm{C}$ NMR (151 MHz, DMSO- $\left.d_{6}\right)$

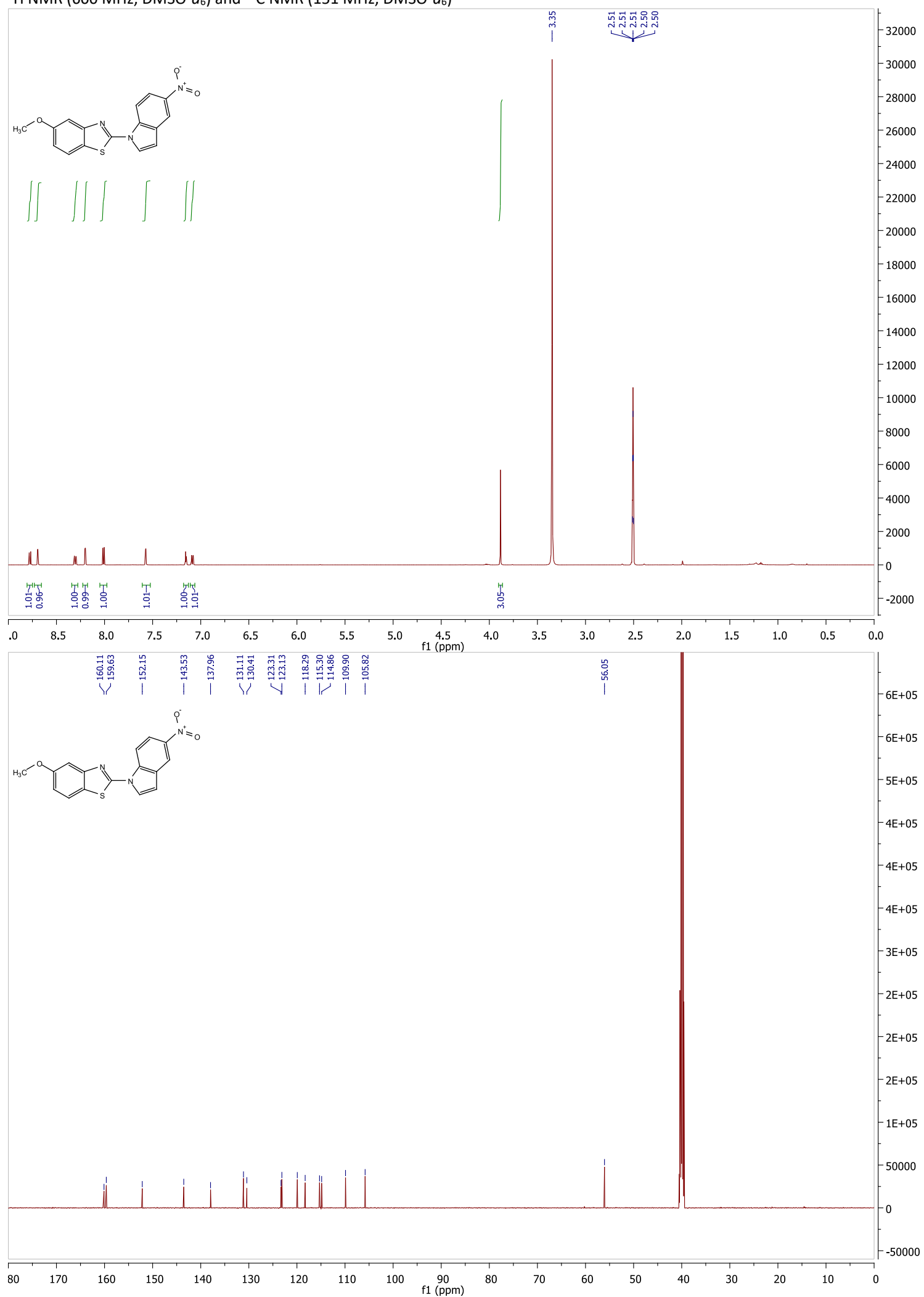


6-fluoro-2-(5-nitro-1H-indol-1-yl)benzo[d]thiazole (4bg)

${ }^{1} \mathrm{H}$ NMR (600 MHz, DMSO- $d_{6}$ ) and ${ }^{13} \mathrm{C}$ NMR (151 MHz, DMSO- $d_{6}$ )

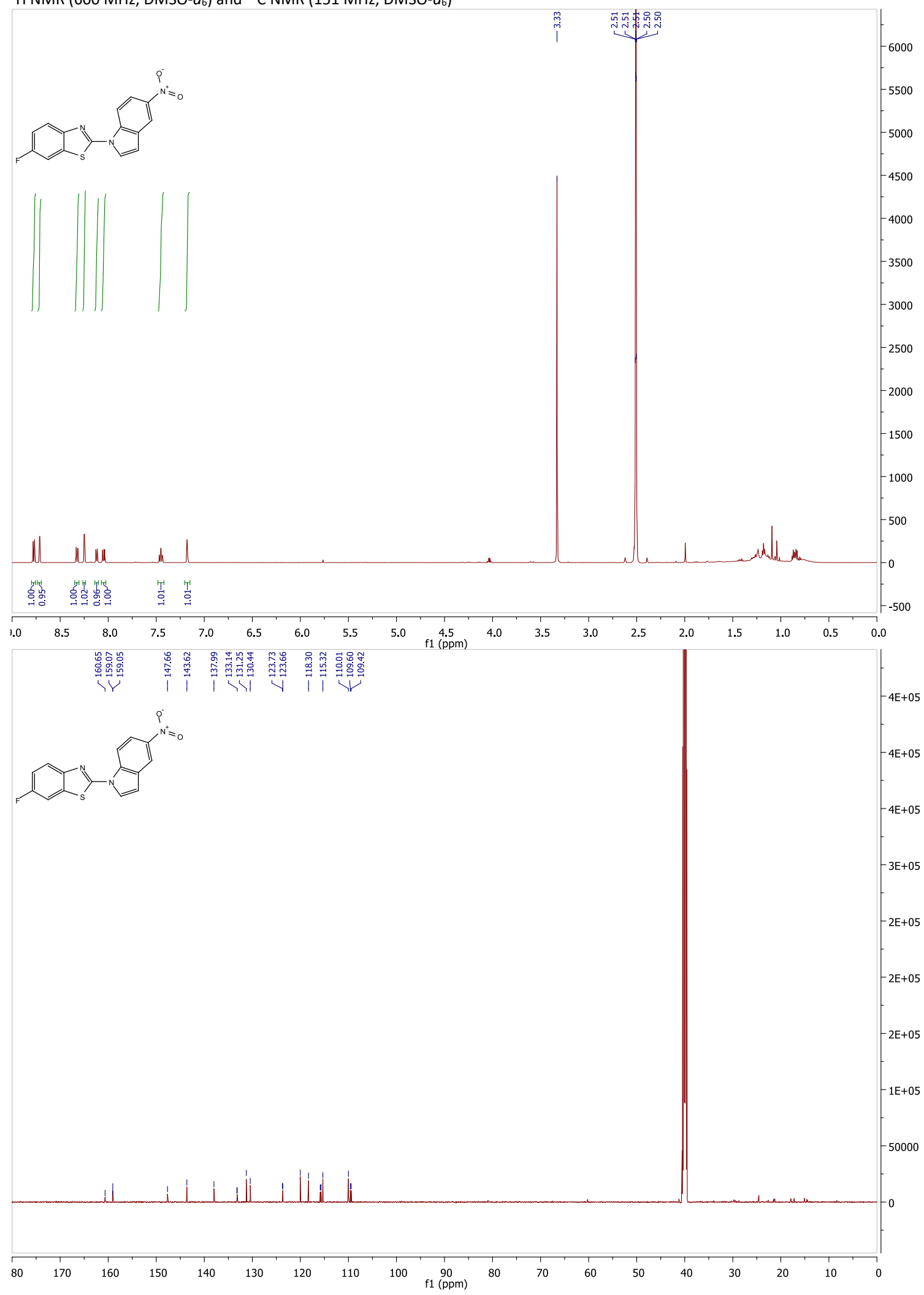


7-bromo-2-(5-nitro-1H-indol-1-yl)benzo[d]thiazole (4bh)

${ }^{1} \mathrm{H}$ NMR $\left(600 \mathrm{MHz}\right.$, DMSO- $d_{6}$ ) and ${ }^{13} \mathrm{C}$ NMR (151 MHz, DMSO- $d_{6}$ )

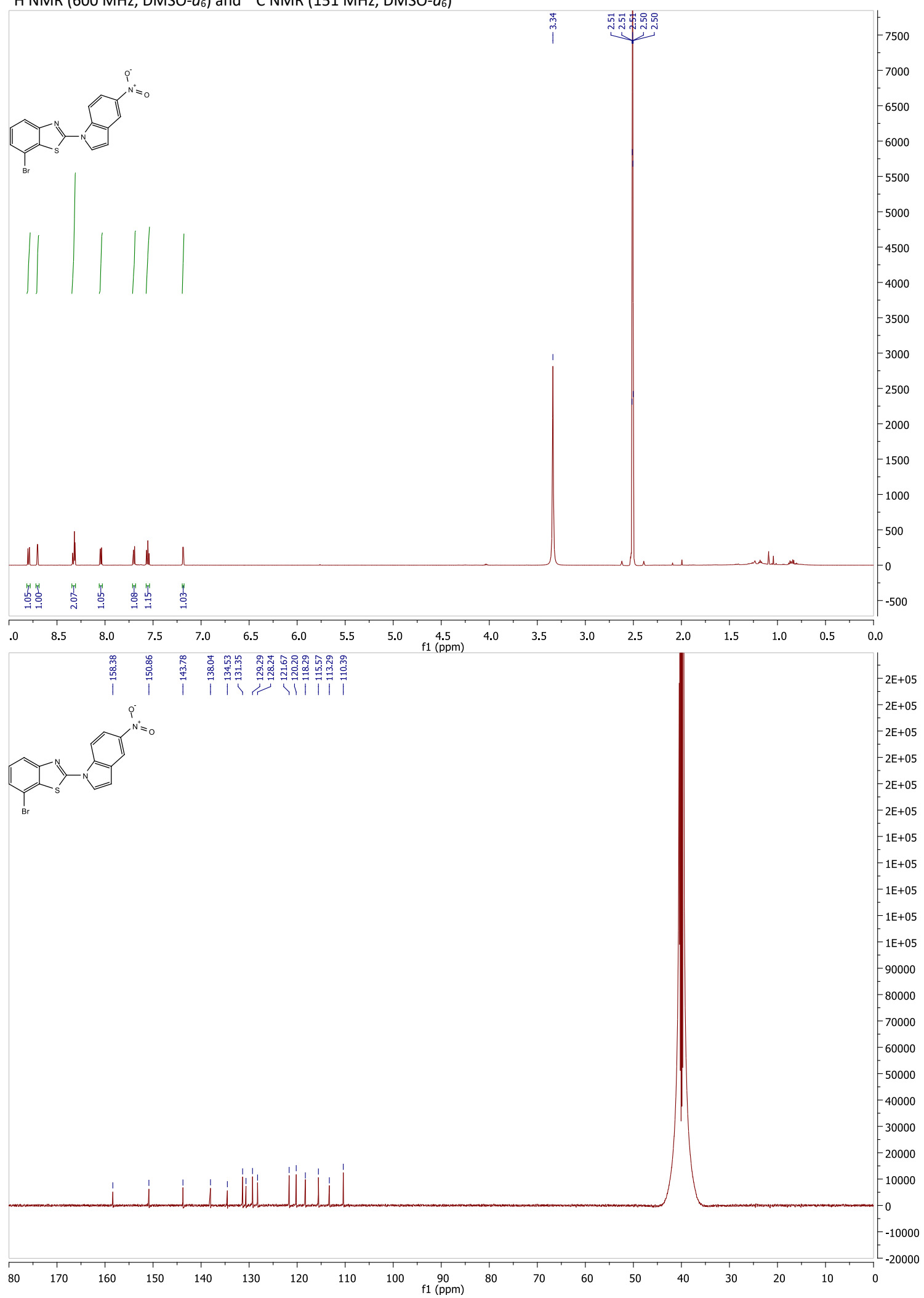


5-bromo-2-(5-nitro-1H-indol-1-yl)benzo[d]thiazole (4bi)

${ }^{1} \mathrm{H}$ NMR (400 MHz, DMSO- $d_{6}$ ) and ${ }^{13} \mathrm{C}$ NMR (151 MHz, DMSO- $d_{6}$ )

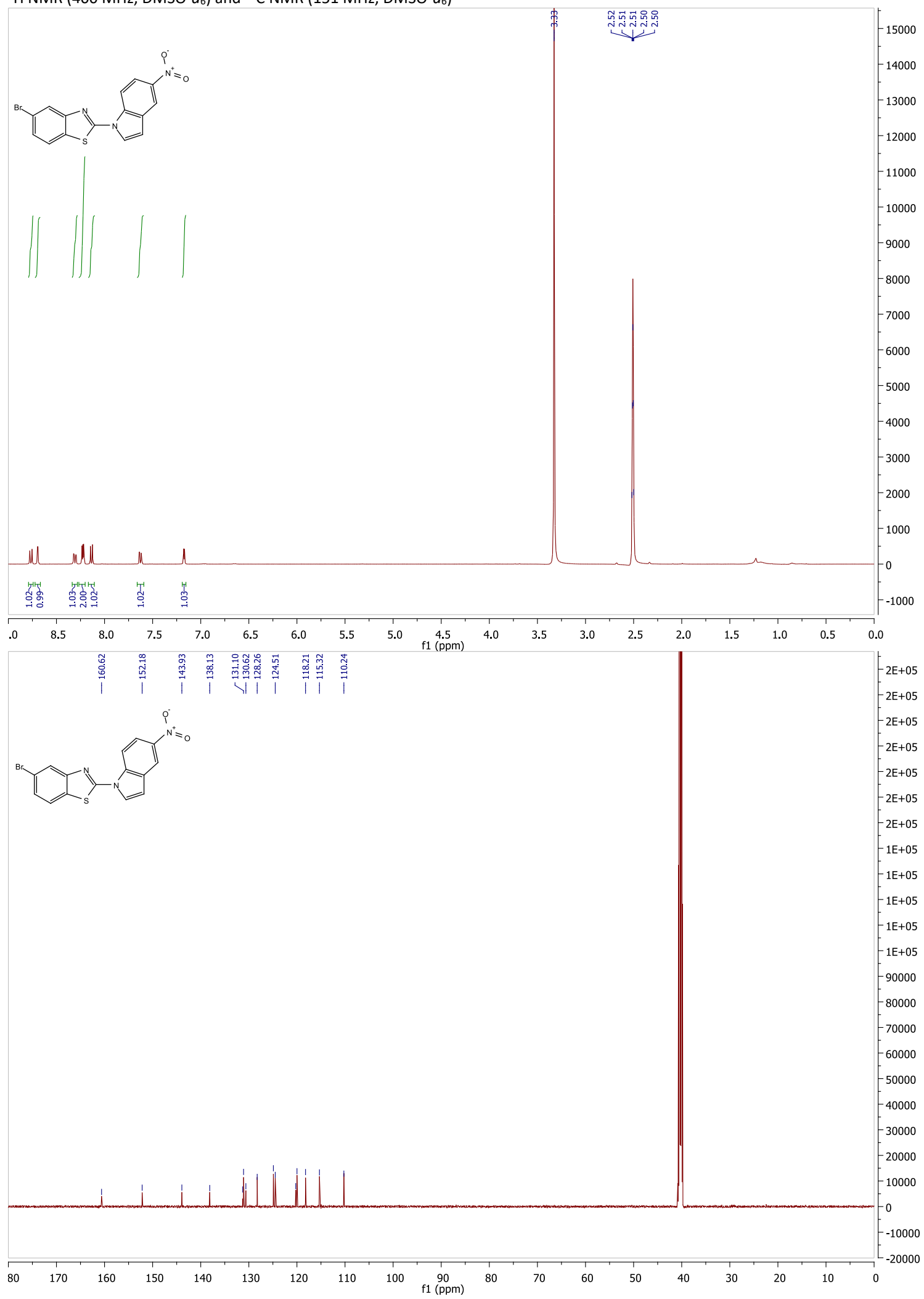


6-chloro-2-(5-nitro-1H-indol-1-yl)benzo[d]thiazole (4bj)

${ }^{1} \mathrm{H}$ NMR $\left(250 \mathrm{MHz}\right.$, DMSO- $\left.d_{6}\right)$ and ${ }^{13} \mathrm{C}$ NMR (63 MHz, DMSO- $\left.d_{6}\right)$

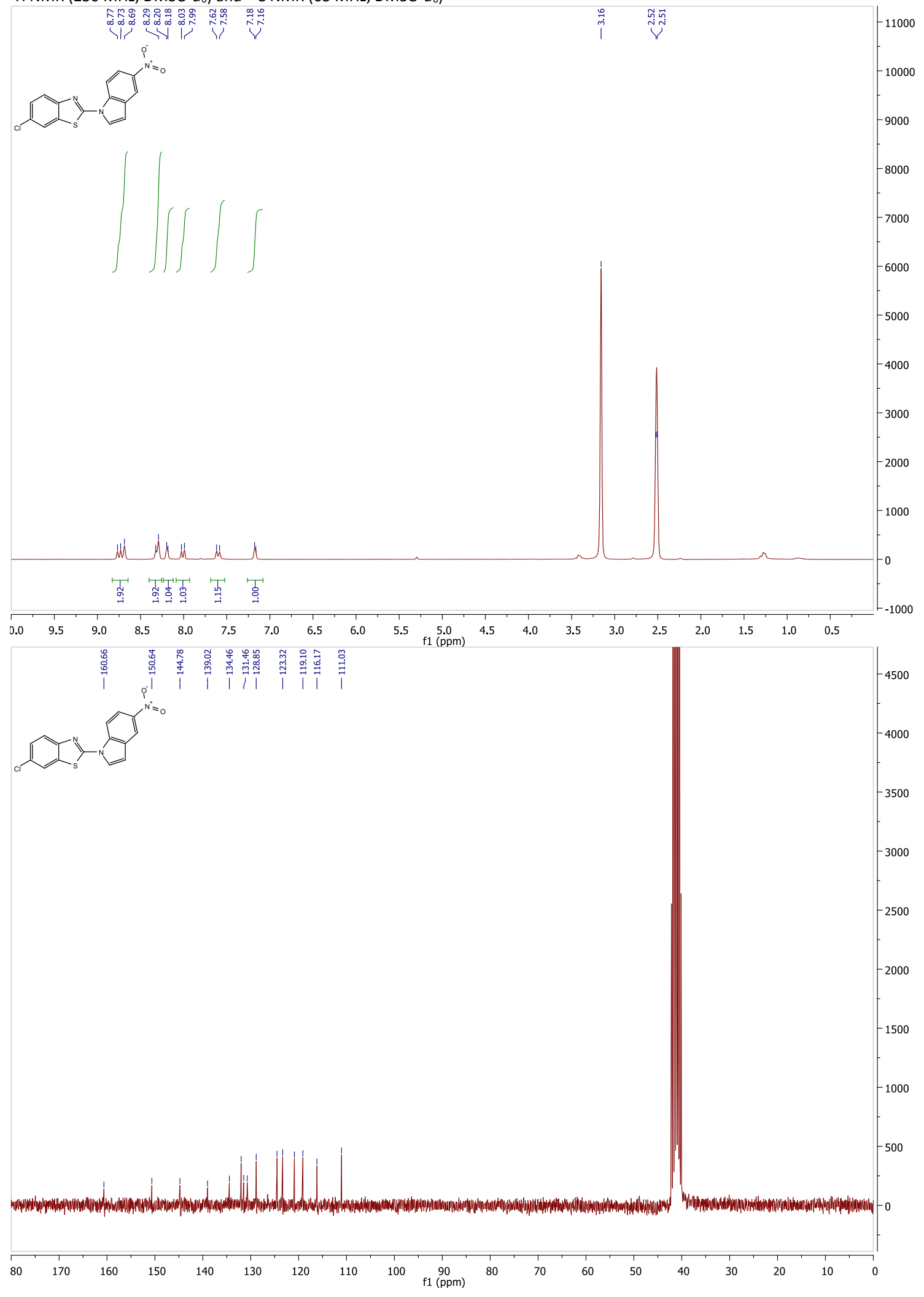




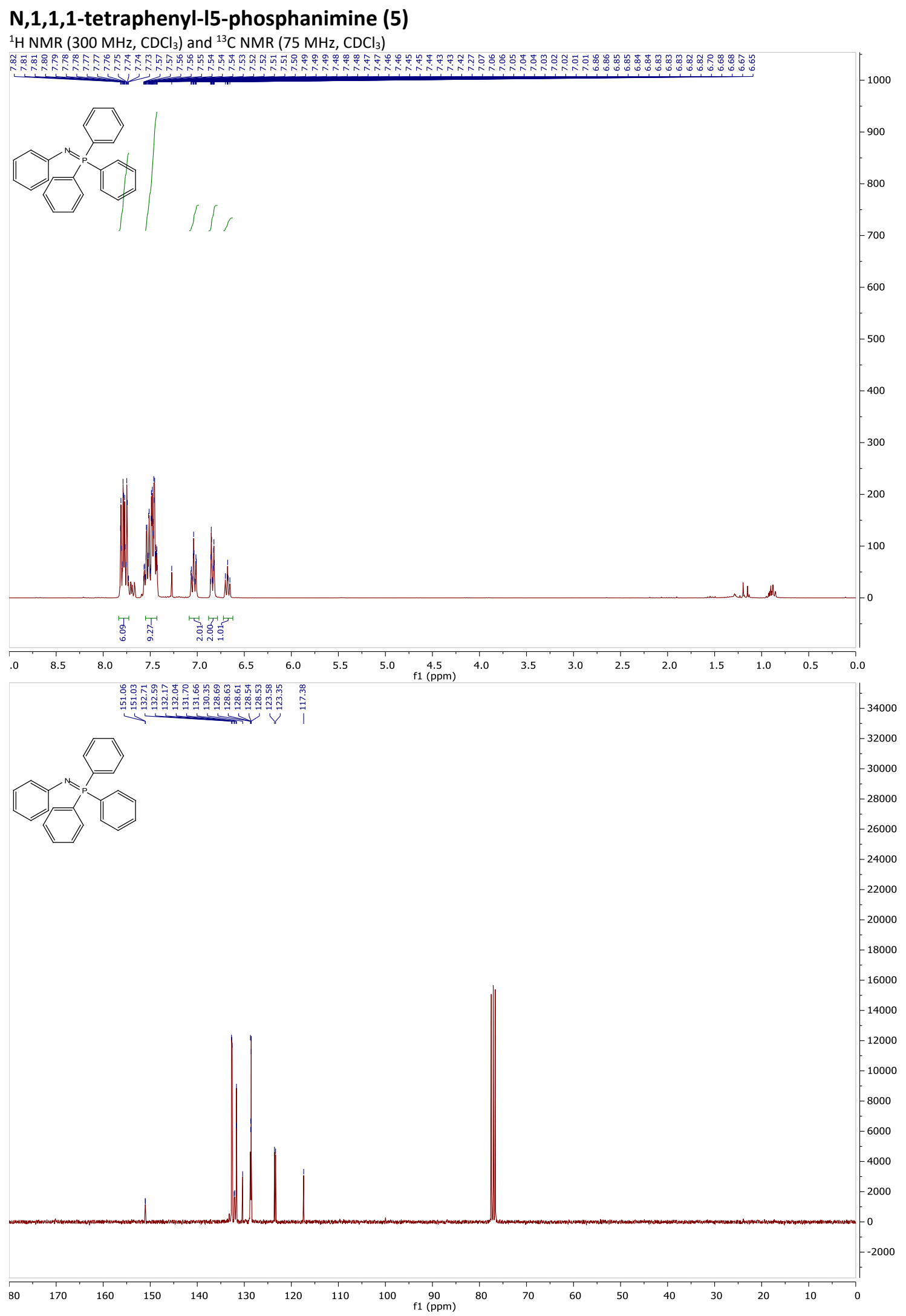

S106 
benzo[d]thiazole-2-d (6a)

${ }^{1} \mathrm{H} \mathrm{NMR}\left(300 \mathrm{MHz}, \mathrm{CDCl}_{3}\right)$ and ${ }^{13} \mathrm{C} \mathrm{NMR}\left(75 \mathrm{MHz}, \mathrm{CDCl}_{3}\right)$

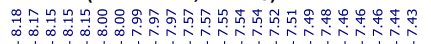
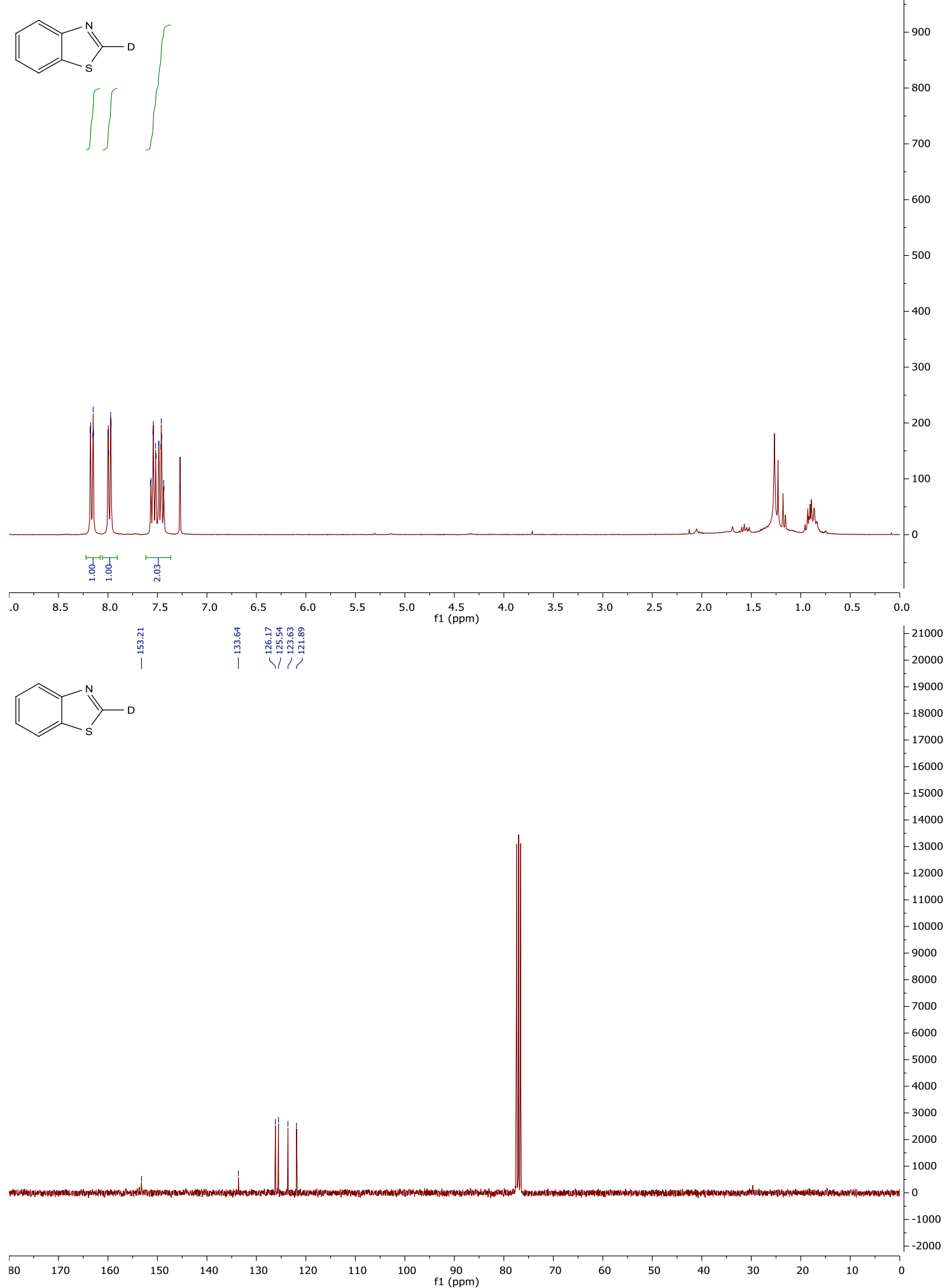
6-methylbenzo[d]thiazole-2-d (6b)

${ }^{1} \mathrm{H} \mathrm{NMR}\left(300 \mathrm{MHz}, \mathrm{CDCl}_{3}\right)$ and ${ }^{13} \mathrm{C} \mathrm{NMR}\left(75 \mathrm{MHz}, \mathrm{CDCl}_{3}\right)$

谓

D
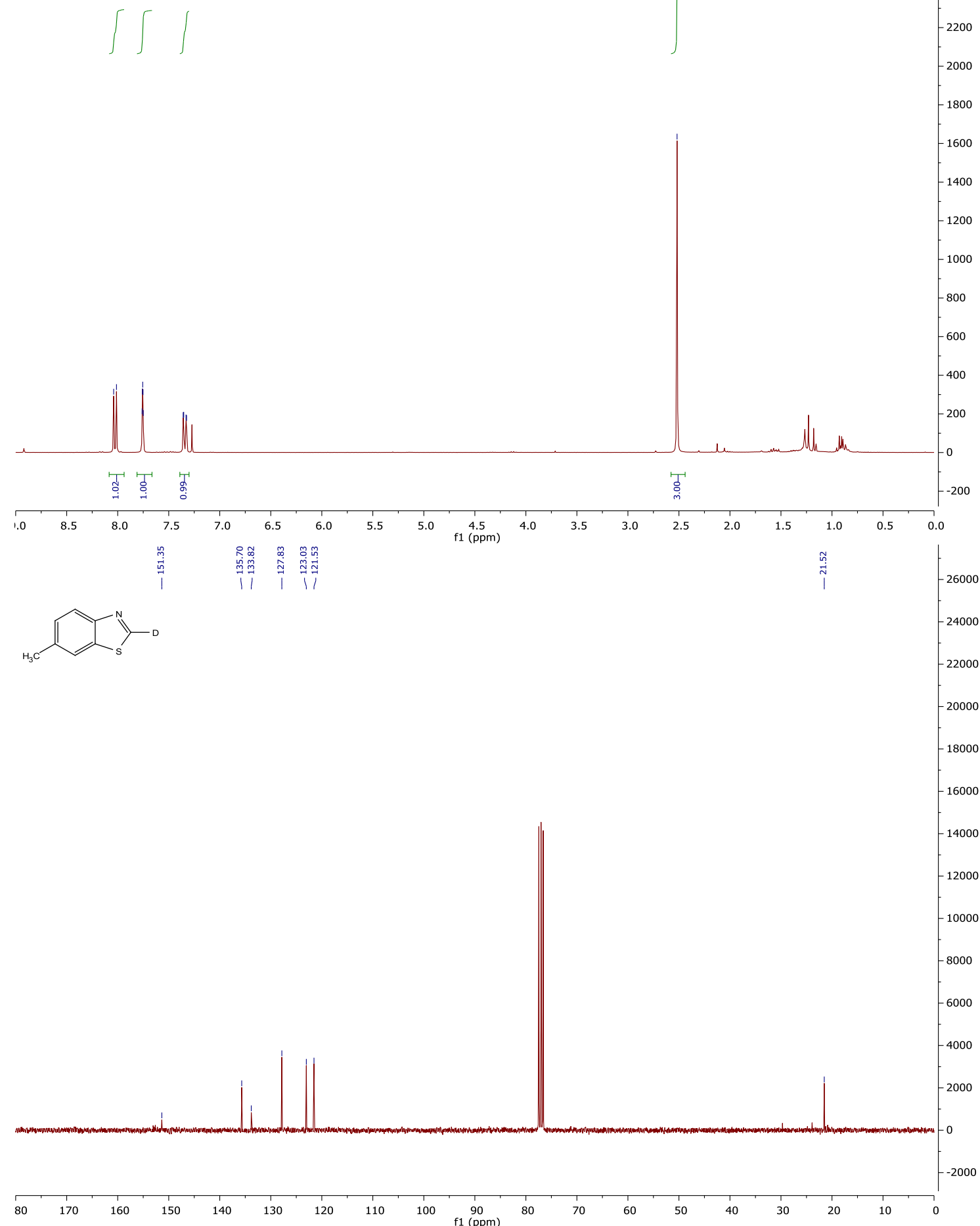


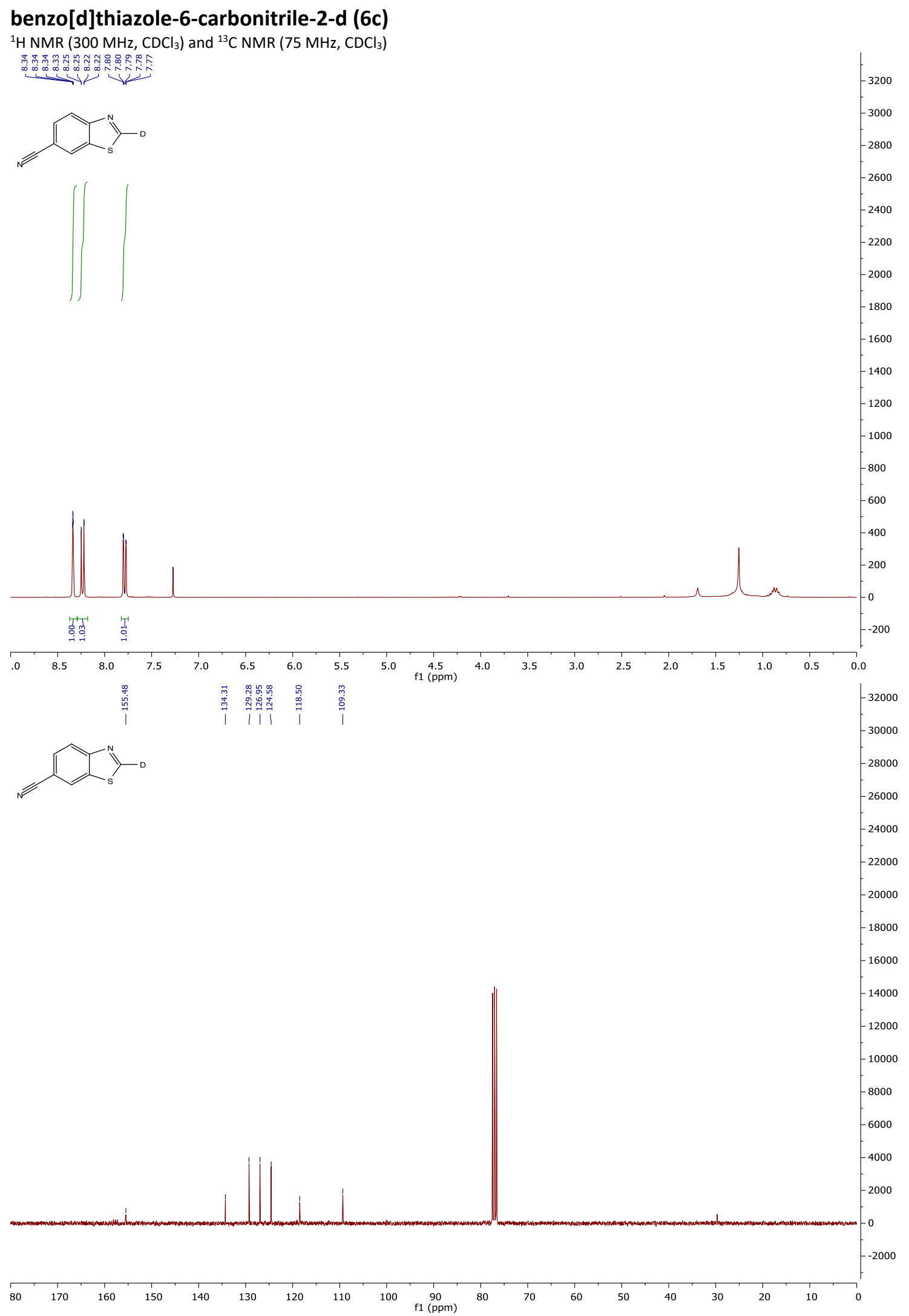

